\title{
Synthetic Studies on Quinine: Quinuclidine Construction via a Ketone Enolate Regio- and Diastereoselective Pd-mediated Allylic Alkylation
}

\author{
Deidre M. Johns, Makoto Mori, Robert M. Williams* \\ Department of chemistry, Colorado State University, Fort Collins, CO 80523-1827 \\ rmw@lamar.colstate.edu
}

\section{Supporting Information}

\section{Table of Contents}

S1 Materials and methods

S2 Experimental procedure for the synthesis of $\mathbf{S - 3 3}$ and $\mathbf{7}$

S3 Experimental procedure for the synthesis of $\mathbf{8}$ and $\mathbf{9}$

S4 Experimental procedure for the synthesis of (+/-)-S-43 and $\mathbf{1 0}$

S5 Experimental procedure for the synthesis of $\mathbf{S - 3 4}$ and $\mathbf{1 1}\left(\mathbf{5}\right.$ with $\left.\mathbf{R}^{\mathbf{1}}=\mathrm{TES}\right)$

S6 Experimental procedure for the synthesis of S-35 and $\mathbf{1 2}$

S7 Experimental procedure for the synthesis of $\mathbf{S - 3 7}$ and $\mathbf{1 5}$

S8 Experimental procedure for the synthesis of $\mathbf{S - 3 8}$ and $\mathbf{S - 3 9}$

S9 Experimental procedure for the synthesis of $\mathbf{1 6}$ and $\mathbf{1 7}$

S10 Experimental procedure for the synthesis of S-40 and $\mathbf{1 9}$

S11 Experimental procedure for the synthesis of $\mathbf{S - 4 2}$

S12 Experimental procedure for the synthesis of $\mathbf{2 0}$ and $\mathbf{2 7}$

S14 Experimental procedure for the synthesis of 2, 29, and S-30

S15 Experimental procedure for the synthesis of 21, 22, 23 and 24

S17 X-ray crystallography data for compound (+/-)-S-43

S27 NMR spectra for selected compounds

General Methods. All air or moisture sensitive reactions were performed under a positive pressured of argon in flame-dried glassware. Tetrahydrofuran (THF), toluene, diethyl ether $\left(\mathrm{Et}_{2} \mathrm{O}\right)$, and $\mathrm{N}, \mathrm{N}$-dimethylformamide (DMF) were obtained from a dry solvent system (activated alumina columns, positive pressure of argon). Dichloromethane, acetonitrile, and triethylamine were distilled from $\mathrm{CaH}_{2}$. Column chromatography was performed on Merck silica gel Kieselgel 60 (230-400 mesh). Melting points were determined in open-end capillary tubes and are uncorrected. ${ }^{1} \mathrm{H}$ NMR and ${ }^{13} \mathrm{C}$ NMR spectra were recorded on Varian 300,400 or $500 \mathrm{MHz}$ spectrometers. Chemical shifts are reported in ppm relative to $\mathrm{CHCl}_{3}$ at $\delta 7.27\left({ }^{1} \mathrm{H} \mathrm{NMR}\right)$ and $\delta 77.23\left({ }^{13} \mathrm{C} \mathrm{NMR}\right)$ or DMSO-d $\mathrm{d}_{5}$ at $\delta 2.5\left({ }^{1} \mathrm{H} \mathrm{NMR}\right)$ and $\delta 39.51\left({ }^{13} \mathrm{C}\right.$ NMR) or MeOH-d $4 \delta 3.31$ ( ${ }^{1} \mathrm{H}$ NMR) and $\delta 49.15\left({ }^{13} \mathrm{C}\right.$ NMR). Mass spectra were obtained on Fisons VG Autospec. IR spectra were obtained from thin films on a NaCl plate using a Perkin-Elmer 1600 series FT-IR spectrometer. Optical rotations were collected at $589 \mathrm{~nm}$ on a Rudolph Research automatic polarimeter Autopol III. 


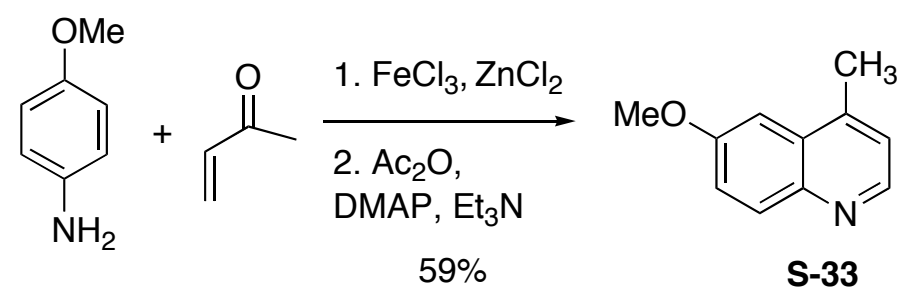

6-Methoxy-4-methylquinoline (S-33): The following procedure was adapted from Wilson's preparation of S-33. ${ }^{1}$ To a solution of zinc(II)chloride $(3.27 \mathrm{~g}, 24 \mathrm{mmol})$ and $p$-methoxyaniline $(23.30 \mathrm{~g}, 189 \mathrm{mmol})$ in ethanol $(100 \mathrm{~mL})$ at $40{ }^{\circ} \mathrm{C}$ was added iron(III)chloride $(81.10 \mathrm{~g}, 500 \mathrm{mmol})$ and the reaction was heated to $80{ }^{\circ} \mathrm{C}$. To the black reaction mixture was added methyl vinyl ketone $\left(14.2 \mathrm{~mL}, 150 \mathrm{mmol}\right.$; dried over $\mathrm{CaCl}_{2}, \mathrm{~K}_{2} \mathrm{CO}_{3}$, and then distilled) in ethanol $(50 \mathrm{~mL})$ dropwise over 1 hour and the reaction was stirred at $80{ }^{\circ} \mathrm{C}$ for an additional 15 hours before cooling to room temperature and quenching with aqueous sodium hydroxide (1L, $1 \mathrm{~N}$ solution). The mixture was filtered through a plug of Florosil resin and rinsed with EtOAc (6L) until no product remained on the resin by TLC. The organic layer was concentrated under reduced pressure. To the crude oil was added THF $(400 \mathrm{~mL})$, triethyl amine $(7.0 \mathrm{~mL}, 50 \mathrm{mmol})$, 4-dimethylamino pyridine $(0.20 \mathrm{~g}, 1.60 \mathrm{mmol})$, and acetic anhydride $(4.7 \mathrm{~mL}, 500$ $\mathrm{mmol})$. The reaction was stirred for two days at room temperature, concentrated under reduced pressure and partitioned between water $(500 \mathrm{~mL})$ and EtOAc $(500 \mathrm{~mL})$. The aqueous layer was extracted with EtOAc $(5 \times 300$ $\mathrm{mL})$. The combined organic layers were washed with $1 \mathrm{~N} \mathrm{HCl}(4 \times 200 \mathrm{~mL})$ and the combined acid washes were neutralized with $\mathrm{Na}_{2} \mathrm{CO}_{3}(\mathrm{~s})$ and $\mathrm{K}_{2} \mathrm{CO}_{3}(\mathrm{~s})$. The product was extracted from the neutralized aqueous layer into EtOAc $(3 \times 200 \mathrm{~mL})$ and the combined organic extracts were washed with brine, dried over $\mathrm{MgSO}_{4}$, filtered through Celite, and concentrated under reduced pressure. The crude oil was purified by flash chromatography $(3: 2$ hexanes/EtOAc) to afford $15.41 \mathrm{~g}(88.50 \mathrm{mmol}, 59 \%)$ of 6-methoxy-4-methylquinoline as a yellow solid. TLC $\mathrm{R}_{\mathrm{f}}=0.21\left(1: 1\right.$ hexanes/EtOAc). Melting point $26-29^{\circ} \mathrm{C}^{2}{ }^{1}{ }^{1} \mathrm{H} \mathrm{NMR}\left(\mathrm{CDCl}_{3}, 400 \mathrm{MHz}\right) \delta 8.58(\mathrm{~d}, J=4 \mathrm{~Hz}, 1 \mathrm{H}), 8.00$ $(\mathrm{d}, J=9 \mathrm{~Hz}, 1 \mathrm{H}), 7.31(\mathrm{dd}, J=3 \mathrm{~Hz}, 9 \mathrm{~Hz}, 1 \mathrm{H}), 7.13-7.10(\mathrm{~m}, 2 \mathrm{H}), 3.89(\mathrm{~s}, 3 \mathrm{H}), 2.58(\mathrm{~s}, 3 \mathrm{H}) .{ }^{13} \mathrm{C} \mathrm{NMR}\left(\mathrm{CDCl}_{3}\right.$, $125 \mathrm{MHz}) \delta 157.7,147.8,144.0,142.9,131.5,129.3,122.2,121.6,101.9,55.6,19.0$. FT-IR (NaCl, neat) 2936, 2832, 1621, 1510, 1475, 1428, 1227, 1031, 845, $736 \mathrm{~cm}^{-1}$. HRMS (FAB+) Calcd for $\mathrm{C}_{11} \mathrm{H}_{12} \mathrm{NO}$ 174.0919. Found $174.0913(\mathrm{M}+\mathrm{H})$.<smiles>COc1ccc2nccc(C)c2c1</smiles>

S-33

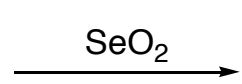<smiles>COc1ccc2nccc(C=O)c2c1</smiles>

7

6-Methoxyquinoline-4-carboxyaldehyde (7). ${ }^{3}$ To a solution of 6-methoxy-4-methylquinoline S-33 (15.28 g, 88.20 $\mathrm{mmol})$ in 1,4-dioxane $(50 \mathrm{~mL})$ was added a solution of selenium dioxide $(14.61 \mathrm{~g}, 131.6 \mathrm{mmol})$ in a 4:1 mixture of 1,4-dioxane $(100 \mathrm{~mL})$ and $\mathrm{H}_{2} \mathrm{O}(25 \mathrm{~mL})$ over 30 minutes at $80{ }^{\circ} \mathrm{C}$. The resulting mixture was stirred at $90{ }^{\circ} \mathrm{C}$ for 15 h. After cooling to room temperature, the mixture was filtered through Celite, and washed with saturated $\mathrm{NaHCO}_{3}$. The aqueous layer was extracted with EtOAc $(3 \times 100 \mathrm{~mL})$. The combined organic layers were washed with brine, dried over anhydrous $\mathrm{Na}_{2} \mathrm{SO}_{4}$, filtered through Celite, and concentrated under reduced pressure. Purification of the crude oil by flash chromatography (1:1 hexanes/EtOAc) gave a brown solid that was recrystallized (hexanes/EtOAc)

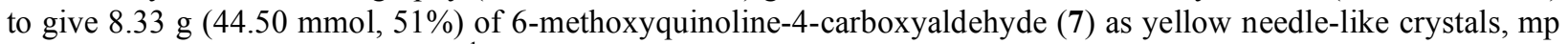
92-97 ${ }^{\circ} \mathrm{C}$. TLC $\mathrm{R}_{\mathrm{f}}=0.47$ (EtOAc). ${ }^{1} \mathrm{H}$ NMR $\left(\mathrm{CDCl}_{3}, 400 \mathrm{MHz}\right) \delta 10.42(\mathrm{~s}, 1 \mathrm{H}), 9.04(\mathrm{~d}, J=4 \mathrm{~Hz}, 1 \mathrm{H}), 8.46(\mathrm{~d}, J=3$ $\mathrm{Hz}, 1 \mathrm{H}), 8.09(\mathrm{~d}, J=9 \mathrm{~Hz}, 1 \mathrm{H}), 7.74(\mathrm{~d}, J=4 \mathrm{~Hz}, 1 \mathrm{H}), 7.46(\mathrm{dd}, J=9 \mathrm{~Hz}, 3 \mathrm{~Hz}, 1 \mathrm{H}), 4.00(\mathrm{~s}, 3 \mathrm{H}) .{ }^{13} \mathrm{C} \mathrm{NMR}$ $\left(\mathrm{CDCl}_{3}, 100 \mathrm{MHz}\right) \delta 193.8,160.7,147.7,146.0,135.4,131.5,127.6,125.4,123.4,102.6,56.0$. IR (NaCl, neat) $3019,2834,2743,2353,1695,1613,1480,1239,1127,1032,854,648 \mathrm{~cm}^{-1}$. HRMS (FAB+) Calcd for $\mathrm{C}_{11} \mathrm{H}_{10} \mathrm{NO}_{2}$ 188.0712. Found $188.0719(\mathrm{M}+\mathrm{H})$.

\footnotetext{
${ }^{1}$ Wilson, S. R.; Grandi, M. J. J. Org. Chem. 1991, 56, 4766.

${ }^{2}$ Reference for melting point $\left(28-32{ }^{\circ} \mathrm{C}\right)$ : Cambell, K. N.; Schaffner, I. J. J. Am. Chem. Soc. 1945, 67, 86.

${ }^{3}$ Biot, C.; Delhaes, L.; Maciejewski, L. A.; Mortuaire, M.; Camus, D.; Dive, D.; Brocard, J. S. Eur. J. Med. Chem. 2000, 35, 707-714.
} 
<smiles>O=C1CN(C(=O)[O-])[C@H](c2ccccc2)[C@H](c2ccccc2)O1</smiles>

6
TBSCl, NaHMDS

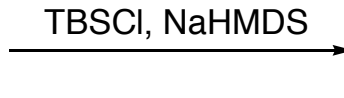

\section{Benzyl (2S,3R)-(+)-6-tert-Butyldimethylsilyloxy-2,3-diphenyl-4-morpholine-5-enecarboxylate (8)}

To a solution of benzyl $(2 S, 3 R)-(+)-6$-oxo-2,3-diphenyl-4-morpholinecarboxylate (6) $(30.00 \mathrm{~g}, 77.44 \mathrm{mmol})$ and tert-butyldimethylsilyl chloride (TBSCl) $(14.0 \mathrm{~g}, 92.9 \mathrm{mmol})$ in THF $(180 \mathrm{~mL})$ at $-78{ }^{\circ} \mathrm{C}$ was added a $1.0 \mathrm{M}$ solution of sodium bis(trimethylsilyl)amide $(93 \mathrm{ml}, 92.9 \mathrm{mmol})$ in THF and the resulting mixture was stirred at the same temperature for $1 \mathrm{~h}$. The mixture was warmed to $25{ }^{\circ} \mathrm{C}$, and concentrated under reduced pressure. The crude oil was filtered through silica gel (gradient hexanes to 1:1 hexanes/EtOAc) to give crude silyl enol ether 8 as a colorless oil. The oil was dissolved in hexanes $(400 \mathrm{~mL})$, and crystallized at $-20{ }^{\circ} \mathrm{C}$. The white solid was collected and washed with cold hexanes to give $37.66 \mathrm{~g}(75.10 \mathrm{mmol}, 97 \%)$ of 8 as a white solid, mp ca. $45-50{ }^{\circ} \mathrm{C}$. TLC $\mathrm{R}_{\mathrm{f}}=0.20$ (10:1 hexanes/EtOAc). ${ }^{1} \mathrm{H}$ NMR $\left(\mathrm{CDCl}_{3}, 400 \mathrm{MHz}\right)$ (rotamers) $\delta$ 7.44-7.31 (m, 3H), 7.25-6.79 (m, $\left.12 \mathrm{H}\right)$, $6.31(\mathrm{~s}, 1 / 2 \mathrm{H}), 6.16(\mathrm{~s}, 1 / 2 \mathrm{H}), 5.44(\mathrm{~m}, 4 \mathrm{H}), 0.97(\mathrm{~s}, 9 \mathrm{H}), 0.24(\mathrm{~s}, 3 / 2 \mathrm{H}), 0.23(\mathrm{~s}, 3 / 2 \mathrm{H}), 0.21(\mathrm{~s}, 3 / 2 \mathrm{H}), 0.20(\mathrm{~s}$, $3 / 2 \mathrm{H}) .{ }^{13} \mathrm{C} \mathrm{NMR}\left(\mathrm{CDCl}_{3}, 100 \mathrm{MHz}\right) \delta 152.3,151.7,147.0,145.9,136.6,136.4,136.3,136.2,135.8,128.7,128.50$, $128.46,128.32,128.28,128.19,128.15,128.1,128.04,127.99,127.9,127.8,127.6,126.6,126.5,88.0,87.1,79.2$, $79.1,67.8,67.5,60.1,59.0,45.6,25.8,22.5,18.2,14.3,-4.0,-4.1,-4.2,-4.3$. IR 3132, 2944, 2874, 1700, 1409, $1355,1297,1255,1095,1001,951,833 \mathrm{~cm}^{-1} . \quad[\mathrm{a}]_{\mathrm{D}}^{20}+14.72\left(c 0.88, \mathrm{CH}_{2} \mathrm{Cl}_{2}\right)$. HRMS (FAB+) Calcd for $\mathrm{C}_{30} \mathrm{H}_{35} \mathrm{NO}_{4} \mathrm{Si}$ 501.2335. Found 501.2340 (M+H).<smiles>COc1ccc2nccc(C=O)c2c1</smiles>

7

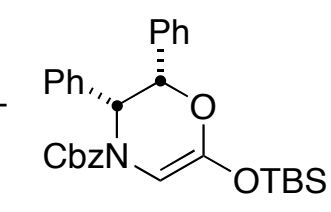

8<smiles></smiles>

Benzyl

$(2 S, 3 R, 5 S)-5-[(1 ' R)$-[hydroxy-4-[6-methoxyquinolyl]]methyl]-6-oxo-2,3-diphenyl-4morpholinecarboxylate (9). To a solution of silyl enol ether $8(2.20 \mathrm{~g}, 4.38 \mathrm{mmol})$ and 6-methoxyquinoline-4carboxyaldehyde (7) $(820 \mathrm{mg}, 4.38 \mathrm{mmol})$ in THF $(30 \mathrm{~mL})$ was added of tetra-butylammonium fluoride (TBAF) $\left(8.78 \mu \mathrm{L}, 8.78 \mathrm{mmol}, 1.0 \mathrm{M}\right.$ solution in THF) at $0{ }^{\circ} \mathrm{C}$ and the resulting mixture was stirred at room temperature for $30 \mathrm{~min}$. To the mixture were added $\mathrm{H}_{2} \mathrm{O}(5 \mathrm{ml})$ and EtOAc $(5 \mathrm{~mL})$, successively. The mixture was stirred vigorously at $25^{\circ} \mathrm{C}$ for $1 \mathrm{~h}$, the white precipitate was collected by filtration, washed with water and EtOAc and dried under vacuum. The white powder $9(1.92 \mathrm{~g}, 3.33 \mathrm{mmol}, 76 \%)$ was carried on without further purification. TLC $\mathrm{R}_{\mathrm{f}}=0.30\left(1: 1\right.$ hexanes/EtOAc). Melting point $198^{\circ} \mathrm{C}(\mathrm{dec}.) .{ }^{1} \mathrm{H}$ NMR $\left(\right.$ DMSO-d $\left._{6}, 400 \mathrm{MHz}, 353 \mathrm{~K}\right) \delta 8.75(\mathrm{~d}, J=5$ $\mathrm{Hz}, 1 \mathrm{H}), 8.21(\mathrm{~s}, 1 \mathrm{H}), 7.99(\mathrm{~d}, J=9 \mathrm{~Hz}, 1 \mathrm{H}), 7.56(\mathrm{~d}, J=4 \mathrm{~Hz}, 1 \mathrm{H}), 7.43(\mathrm{dd}, J=9 \mathrm{~Hz}, 2 \mathrm{~Hz}, 1 \mathrm{H}), 7.30-7.21(\mathrm{~m}$, 3H), 7.20-7.10 (m, 4H), 7.09-7.01 (m, 4H), 6.90-6.81 (m, 3H), 6.79 (d, $J=3 \mathrm{~Hz}, 1 \mathrm{H}), 6.56(\mathrm{~d}, J=7 \mathrm{~Hz}, 2 \mathrm{H}), 6.13$ $(\mathrm{d}, J=7 \mathrm{~Hz}, 2 \mathrm{H}), 6.13(\mathrm{br} \mathrm{s}, 1 \mathrm{H}), 5.41(\mathrm{~d}, J=3 \mathrm{~Hz}, 1 \mathrm{H}), 5.32(\mathrm{~s}, 1 \mathrm{H}), 5.07(\mathrm{AB}, J=12 \mathrm{~Hz}, \Delta v=39 \mathrm{~Hz}, 2 \mathrm{H}), 3.99$ (s, $3 \mathrm{H}) .{ }^{13} \mathrm{C}$ NMR (DMSO-d $6,75 \mathrm{MHz}$, ambient temp. rotamers) $\delta 164.9,157.5,154.4,147.2,144.6,143.4,136.2$, 135.7, 134.6, 131.0, 128.1, 127.9 (2C), 127.6, 127.5, 127.3, 127.1, 126.7, 126.0, 125.9, 121.3, 119.2, 102.1, 78.5, 69.2, 66.9, 62.4, 59.6, 55.9. IR(NaCl, neat) 3516, 3026, 2942, 1731, 1705, 1617, 1445, 1395, 1217, 1103, 1080, $970,703 \mathrm{~cm}^{-1}$. [a $]_{\mathrm{D}}^{20}+33.3$ (c 0.54, DMSO). HRMS (FAB+) Calcd for $\mathrm{C}_{35} \mathrm{H}_{31} \mathrm{~N}_{2} \mathrm{O}_{6}: 575.2182$. Found 575.2182 $(\mathrm{M}+\mathrm{H})$. The relative stereochemistry of $\mathbf{9}$ was determined by X-ray crystallographic analysis of the racemic, acetylated derivative (+/-)-S-43, as X-ray quality crystals of $\mathbf{9}, \mathbf{1 0}$, and $\mathbf{S - 3 4}$ could not be obtained. 


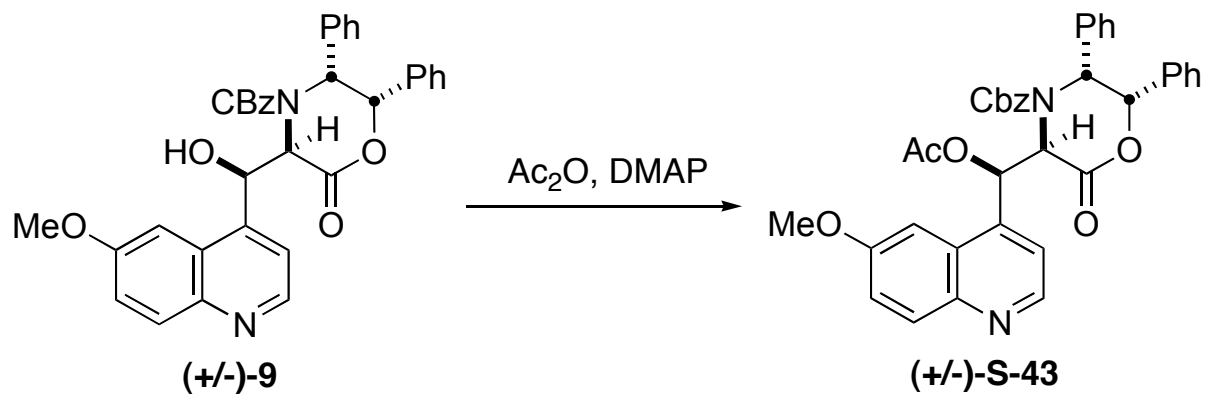

Benzyl $\quad(2 S, 3 R, 5 S)-5-[(1 ' R)$-[acetoxy-4-[6-methoxyquinolyl]]methyl]-6-oxo-2,3-diphenyl-4morpholinecarboxylate ((+/-)-S-43). To a solution of the racemic aldol product (+/-)-9 (15 mg, $26.2 \mu \mathrm{mol})$ in acetic anhydride $(100 \mu \mathrm{L})$ and $\mathrm{CH}_{2} \mathrm{Cl}_{2}(500 \mu \mathrm{L})$ was added DMAP $(10 \mathrm{mg}, 82 \mu \mathrm{mol})$, and the reaction was stirred for 16 hours at room temperature. After which time the solution was diluted with additional $\mathrm{CH}_{2} \mathrm{Cl}_{2}$ and washed with saturated $\mathrm{NaHCO}_{3} a q$, and brine, dried over $\mathrm{Na}_{2} \mathrm{SO}_{4}$, and concentrated. The crude oil was purified by silica gel chromatography (eluted with a solvent gradient of 10:1 to 2:1 hexanes/EtOAc), and then recrystallized from benzene $/ \mathrm{Et}_{2} \mathrm{O}$ to afford off-white X-ray quality crystals of acetylated aldol product (+/-)-S-43 $(10 \mathrm{mg}, 16.2 \mu$ mol, 62\%). ${ }^{1} \mathrm{H}$ NMR $\left(\mathrm{CDCl}_{3}, 400 \mathrm{MHz}\right) \delta 8.76(\mathrm{~d}, J=5 \mathrm{~Hz}, 1 \mathrm{H}), 8.29(\mathrm{~m}, 1 \mathrm{H}), 8.12(\mathrm{~m}, 1 \mathrm{H}), 7.45(\mathrm{dd}, J=9 \mathrm{~Hz}, 2 \mathrm{~Hz}$, $1 \mathrm{H}), 7.35-7.18(\mathrm{~m}, 7 \mathrm{H}), 7.14(\mathrm{t}, J=8 \mathrm{~Hz}, 2 \mathrm{H}), 7.08(\mathrm{t}, J=8 \mathrm{~Hz}, 2 \mathrm{H}), 6.99(\mathrm{~d}, J=7 \mathrm{~Hz}, 2 \mathrm{H}), 6.75(\mathrm{~d}, J=8 \mathrm{~Hz}, 2 \mathrm{H})$, $6.51(\mathrm{~d}, J=8 \mathrm{~Hz}, 2 \mathrm{H}), 6.33(\mathrm{~d}, J=3 \mathrm{~Hz}, 1 \mathrm{H}), 5.54(\mathrm{~d}, J=3 \mathrm{~Hz}, 1 \mathrm{H}), 5.23(\mathrm{~d}, J=3 \mathrm{~Hz}, 1 \mathrm{H}), 5.07(\mathrm{AB} J=12 \mathrm{~Hz}$, $\Delta v=69 \mathrm{~Hz}, 2 \mathrm{H}), 4.0(\mathrm{~s}, 3 \mathrm{H}), 2.38(\mathrm{~s}, 3 \mathrm{H}) .{ }^{13} \mathrm{C}$ NMR $\left(\mathrm{CDCl}_{3}, 100 \mathrm{MHz}\right) \delta 169.0,164.2,159.3,155.4,135.5,135.4$, $134.2,129.1,128.6,128.5,128.4,128.32,128.28,127.8,127.6,126.7,123.6,118.4,101.8,94.6,80.0,72.5,68.5$, 60.8, 60.7, 56.6, 21.6. IR (NaCl, neat) 4314, 3065, 3034, 2936, 1757, 1701, 1621, 1509, 1454, 1397, 1350, 1276, $1225,1117,1067,732,701 \mathrm{~cm}^{-1}$. HRMS (FAB+) Calcd for $\mathrm{C}_{37} \mathrm{H}_{33} \mathrm{~N}_{2} \mathrm{O}_{7}: 617.2288$. Found $617.2258(\mathrm{M}+\mathrm{H})$. Stereochemistry confirmed by X-ray crystallography.<smiles></smiles><smiles>CCOC(c1ccnc2ccc(OC)cc12)[C@]12OC(=O)N1[C@@H](c1ccccc1)[C@@H](c1ccccc1)OC2=O</smiles>

Benzyl (2S,3R,5S)-5-[(1'R)-[triethylsilyloxy-4-[6-methoxyquinolyl]]methyl]-6-oxo-2,3-diphenyl-4morpholinecarboxylate (10). To a suspension of benzyl $(2 S, 3 R, 5 S)-5-\left[\left(1^{\prime} R\right)\right.$-[hydroxy-4-[6methoxyquinolyl]]methyl]-6-oxo-2,3-diphenyl-4-morpholinecarboxylate (9) $(1.90 \mathrm{~g}, 3.31 \mathrm{mmol})$ in a 1:1 mixture of $\mathrm{CH}_{2} \mathrm{Cl}_{2} / \mathrm{THF}(20 \mathrm{ml})$ at $25^{\circ} \mathrm{C}$ was added DMAP $(1.64 \mathrm{~g}, 13.40 \mathrm{mmol})$ and triethylsilyl trifluoromethanesulfonate $(3.0 \mathrm{~mL}, 13.30 \mathrm{mmol})$, successively. The resulting mixture was heated to reflux for $12 \mathrm{~h}$ or until the mixture was clear, cooled to $25^{\circ} \mathrm{C}$, and $\mathrm{H}_{2} \mathrm{O}$ and EtOAc were added. The organic layer was separated and washed with saturated $\mathrm{NaHCO}_{3}$ and brine, dried over anhydrous $\mathrm{Na}_{2} \mathrm{SO}_{4}$, and concentrated. The crude oil was purified by column chromatography on silica gel (3:2 hexanes/EtOAc) to afford $2.27 \mathrm{~g}(3.28 \mathrm{mmol}, 99 \%)$ of benzyl $(2 S, 3 R, 5 S)-5$ [(l'R)-[triethylsilyloxy-4-[6-methoxyquinolyl]]methyl]-6-oxo-2,3-diphenyl-4-morpholinecarboxylate (10) as a white amorphous solid. TLC $\mathrm{R}_{\mathrm{f}}=0.36\left(3: 2\right.$ hexanes/EtOAc). ${ }^{1} \mathrm{H} \mathrm{NMR}\left(\mathrm{CDCl}_{3}, 400 \mathrm{MHz}\right) \delta 8.80(\mathrm{~d}, J=5 \mathrm{~Hz}, 1 \mathrm{H})$, $8.17(\mathrm{~d}, J=3 \mathrm{~Hz}, 1 \mathrm{H}), 8.08(\mathrm{~d}, J=9 \mathrm{~Hz}, 1 \mathrm{H}), 7.54(\mathrm{~d}, J=5 \mathrm{~Hz}, 1 \mathrm{H}), 7.42(\mathrm{dd}, J=9 \mathrm{~Hz}, 3 \mathrm{~Hz}, 1 \mathrm{H}), 7.31-7.15(\mathrm{~m}$, $7 \mathrm{H}), 7.06$ (t, $J=8 \mathrm{~Hz}, 2 \mathrm{H}), 6.98$ (br d, $J=8 \mathrm{~Hz}, 2 \mathrm{H}), 6.85$ (br d, $J=8 \mathrm{~Hz}, 2 \mathrm{H}), 6.63$, (d, $J=3 \mathrm{~Hz}, 1 \mathrm{H}), 6.53$ (br d, $J=8 \mathrm{~Hz}, 2 \mathrm{H}), 6.35$ (br d, $J=2 \mathrm{~Hz}, 1 \mathrm{H}), 5.38$ (br d, $J=3 \mathrm{~Hz}, 1 \mathrm{H}), 5.23$ (br d, $J=3 \mathrm{~Hz}, 1 \mathrm{H}), 5.04(\mathrm{AB}, J=13 \mathrm{~Hz}$, $\Delta v=31 \mathrm{~Hz}, 2 \mathrm{H}) 4.08(\mathrm{~s}, 3 \mathrm{H}), 0.94(\mathrm{t}, J=8 \mathrm{~Hz}, 9 \mathrm{H}), 0.65(\mathrm{q}, J=8 \mathrm{~Hz}, 6 \mathrm{H}) .{ }^{13} \mathrm{C} \mathrm{NMR}\left(\mathrm{CDCl}_{3}, 100 \mathrm{MHz}\right) \delta 165.2$, $158.8,155.3,147.6,144.5,144.0,136.0135 .8,134.9,132.0,128.7,128.5,128.4,128.3,128.2,128.0$ (2C), 127.7 , 126.6, 126.5, 122.3, 119.7, 101.5, 79.3, 71.5, 68.2, 63.2, 61.0, 56.4, 6.9, 4.9. IR (NaCl, neat) 3032, 2957, 2878, $1758,1697,1621,1510,1454,1400,1353,1282,1229,1117,747,699 \mathrm{~cm}^{-1} .[\alpha]^{25}-5.1\left(c 1.33, \mathrm{CHCl}_{3}\right)$. HRMS (FAB+) Calcd for $\mathrm{C}_{41} \mathrm{H}_{45} \mathrm{~N}_{2} \mathrm{O}_{6} \mathrm{Si}$ : 689.3047. Found $689.3054(\mathrm{M}+\mathrm{H})$. Anal. calc. for $\mathrm{C}_{41} \mathrm{H}_{44} \mathrm{~N}_{2} \mathrm{O}_{6} \mathrm{Si}$ : C, 71.48; $\mathrm{H}$, 6.44; N, 4.07. Found: C, 71.08; H, 6.46; N, 4.25. 
<smiles>CCOC(c1ccnc2ccc(OC)cc12)C1C(=O)O[C@@H](c2ccccc2)[C@H](c2ccccc2)N1C(=O)OC</smiles>

10<smiles>CCOC(c1ccnc2ccc(OC)cc12)[C@@H]1N[C@@H](c2ccccc2)[C@H](c2ccccc2)OC1=O</smiles>

S-34

Methyl (2R,3R)-2-tert-Butoxycarbonylamino-3-(6-methoxyquinol-3-yl)-3-triethylsilyloxy-propionate (S-34). To a solution of benzyl $(2 S, 3 R, 5 S)$-5-[(1'R)-[triethylsilyloxy-4-[6-methoxyquinolyl]]methyl]-6-oxo-2,3-diphenyl-4morpholinecarboxylate (10) $(2.27 \mathrm{~g}, 3.30 \mathrm{mmol})$ in EtOAc $(35 \mathrm{~mL})$ was added $10 \%$ Pd-C $(250 \mathrm{mg})$, and the mixture was stirred under $\mathrm{H}_{2}$ (atmospheric pressure) at room temperature for $12 \mathrm{~h}$. The catalyst was removed by filtration through Celite and washed with $\mathrm{MeOH}$. The filtrate was concentrated under reduced pressure to obtain $\mathbf{S - 3 4}$ as colorless oil, which was used without further purification. A small sample was recrystallized from hexanes/ $\mathrm{Et}_{2} \mathrm{O}$ for analysis. Melting point $148-150{ }^{\circ} \mathrm{C} .{ }^{1} \mathrm{H} \mathrm{NMR}\left(\mathrm{CDCl}_{3}, 400 \mathrm{MHz}\right) \delta 8.70(\mathrm{~d}, J=5 \mathrm{~Hz}, 1 \mathrm{H}), 8.07(\mathrm{~d}, J=9 \mathrm{~Hz}, 1 \mathrm{H})$, $7.62(\mathrm{~d}, J=5 \mathrm{~Hz}, 1 \mathrm{H}), 7.59(\mathrm{br} \mathrm{s}, 1 \mathrm{H}), 7.39(\mathrm{dd}, J=9 \mathrm{~Hz}, 3 \mathrm{~Hz}, 1 \mathrm{H}), 7.25-7.07(\mathrm{~m}, 6 \mathrm{H}), 6.74(\mathrm{br} \mathrm{d}, J=8 \mathrm{~Hz}, 2 \mathrm{H})$, 6.55 (br d, $J=8 \mathrm{~Hz}, 2 \mathrm{H}), 6.09$ (br d, $J=3 \mathrm{~Hz}, 1 \mathrm{H}), 5.09$ (d, $J=4 \mathrm{~Hz}, 1 \mathrm{H}), 4.64$ (d, $J=3 \mathrm{~Hz}, 1 \mathrm{H}), 3.91$ (s, 3H), $3.64(\mathrm{~d}, J=4 \mathrm{~Hz}, 1 \mathrm{H}), 0.91(\mathrm{t}, J=8 \mathrm{~Hz}, 9 \mathrm{H}), 0.62(\mathrm{qd}, J=8 \mathrm{~Hz}, 3 \mathrm{~Hz}, 6 \mathrm{H}) .{ }^{13} \mathrm{C} \mathrm{NMR}\left(\mathrm{CDCl}_{3}, 100 \mathrm{MHz}\right) \delta 173.6$, 168.3 158.3 , 147.5, 144.8, 144.7, 136.9, 134.8, 131.8, 128.4 (2C's), 127.8 (2C's), 127.5 (2C's), 122.5, 120.7, 102.3, 94.6, 85.0, 64.3, 58.1, 55.8, 7.0, 4.9. IR (NaCl, neat) 3344, 3032, 2950, 2889, 1734, 1619, 1504, 1462, 1357, 1233, 1087, 1020, 848, $735 \mathrm{~cm}^{-1}$. $[\alpha]^{25}-125.1\left(c\right.$ 4.47, $\left.\mathrm{CHCl}_{3}\right)$. HRMS (FAB+) Calcd for $\mathrm{C}_{33} \mathrm{H}_{39} \mathrm{~N}_{2} \mathrm{O}_{4} \mathrm{Si}$ : 555.2679. Found 555.2678 (M+H). Anal. Calc. for $\mathrm{C}_{33} \mathrm{H}_{38} \mathrm{~N}_{2} \mathrm{O}_{4} \mathrm{Si}$ : C, 71.45; H, 6.90; N, 5.05. Found: C, 71.06; H, 7.17; N, 5.30 .<smiles>CCOC(c1ccnc2ccc(OC)cc12)[C@H]1N[C@@H](c2ccccc2)[C@H](c2ccccc2)OC1=O</smiles>

S-34

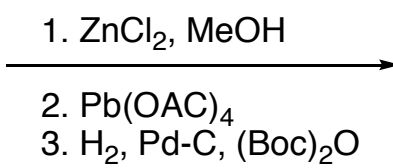

3. $\mathrm{H}_{2}, \mathrm{Pd}-\mathrm{C},(\mathrm{Boc})_{2} \mathrm{O}$<smiles>CCOC(=O)NC(c1ccnc2ccc(OC)cc12)C(NC(=O)OC)OCC</smiles>

(2R,3R)-methyl 2-(tert-butoxycarbonyl)-3-(6-methoxyquinolin-4-yl)-3-(triethylsilyloxy)propanoate (11). To crude S-34 dissolved in absolute $\mathrm{MeOH}(30 \mathrm{~mL})$ was added anhydrous $\mathrm{ZnCl}_{2}(2.25 \mathrm{~g}, 16.5 \mathrm{mmol})$. The reaction mixture was stirred at $50{ }^{\circ} \mathrm{C}$ for $24 \mathrm{~h}$, and then cooled to room temperature and aqueous $10 \% \mathrm{Na}_{2} \mathrm{CO}_{3}$ solution (30 $\mathrm{mL})$ and EtOAc $(60 \mathrm{~mL})$ were added. After stirring for 10 minutes, the mixture was filtered through Celite. The organic layer was separated, dried over $\mathrm{Na}_{2} \mathrm{SO}_{4}$, and concentrated under reduced pressure. The residual oil was filtered through a short column of silica gel $\left(10 \mathrm{~g}\right.$, eluted with EtOAc). Methyl ester was obtained as white solid. ${ }^{1} \mathrm{H}$ $\operatorname{NMR}\left(\mathrm{CDCl}_{3}, 300 \mathrm{MHz}\right) \delta 8.72(\mathrm{~d}, J=6 \mathrm{~Hz}, 1 \mathrm{H}), 8.08(\mathrm{~d}, J=9 \mathrm{~Hz}, 1 \mathrm{H}), 7.42(\mathrm{dd}, J=9 \mathrm{~Hz}, 2 \mathrm{~Hz}, 1 \mathrm{H}), 7.21-7.03$ (m, 6H), 6.90-6.85 (m, 2H), $6.73(\mathrm{~d}, J=8 \mathrm{~Hz}, 2 \mathrm{H}), 5.36(\mathrm{br} \mathrm{s}, 1 \mathrm{H}), 4.49(\mathrm{br} \mathrm{s}, 1 \mathrm{H}), 3.91(\mathrm{~s}, 3 \mathrm{H}), 3.71(\mathrm{br} \mathrm{s}, 1 \mathrm{H})$, $3.48(\mathrm{br} \mathrm{s}, 1 \mathrm{H}), 3.38(\mathrm{~s}, 3 \mathrm{H}), 2.30(\mathrm{br} \mathrm{s}, 1 \mathrm{H}), 1.95(\mathrm{br} \mathrm{s}, 1 \mathrm{H}), 0.77(\mathrm{t}, J=8 \mathrm{~Hz}, 9 \mathrm{H}), 0.42(\mathrm{q}, J=8 \mathrm{~Hz}, 6 \mathrm{H})$. IR (NaCl, neat) 2953, 2910, 2877, 1739, 1621, 1509, 1473, 1454, $1100 \mathrm{~cm}^{-1} .[\alpha]_{\mathrm{D}}^{25}-45\left(c 0.94, \mathrm{CDCl}_{3}\right)$. HRMS $(\mathrm{FAB}+)$ Calcd for $\mathrm{C}_{34} \mathrm{H}_{43} \mathrm{~N}_{2} \mathrm{O}_{5} \mathrm{Si}$ : 587.2941. Found 587.2936 (M+H).

To a solution of crude methyl ester dissolved in a $1: 1$ mixture of $\mathrm{MeOH} / \mathrm{CH}_{2} \mathrm{Cl}_{2}(30 \mathrm{~mL})$ was added $\mathrm{Pb}(\mathrm{OAc})_{4}(2.20 \mathrm{~g}, 5.00 \mathrm{mmol})$ at $0{ }^{\circ} \mathrm{C}$. After stirring for 5 minutes at $0{ }^{\circ} \mathrm{C}$, saturated $\mathrm{NaHCO}_{3}$ and EtOAc were added to the mixture. The separated organic layer was washed with $\mathrm{H}_{2} \mathrm{O}$ and brine, dried over $\mathrm{Na}_{2} \mathrm{SO}_{4}$, and concentrated under reduced pressure. To the residual oil dissolved in EtOAc $(40 \mathrm{~mL})$ was added $(\mathrm{Boc})_{2} \mathrm{O}(1.10 \mathrm{~g}$, $5.00 \mathrm{mmol})$ and $10 \% \mathrm{Pd}-\mathrm{C}(500 \mathrm{mg})$. The resulting mixture was stirred under atmospheric pressure of $\mathrm{H}_{2}$ at room temperature for $24 \mathrm{~h}$. The catalyst was removed by filtration through Celite, rinsed with EtOAc, and concentrated 
under reduced pressure. The residue was purified by flash chromatography on silica gel (60 g, eluted with 3:2 hexanes/EtOAc) followed by recrystallization from hexanes to afford $1.28 \mathrm{~g}(2.61 \mathrm{mmol}, 79 \%$, from $\mathbf{1 0})$ of $\mathbf{1 1}$ as white solid. Melting point $92-95^{\circ} \mathrm{C} .{ }^{1} \mathrm{H} N M R\left(\mathrm{CDCl}_{3}, 400 \mathrm{MHz}\right) \delta 8.76(\mathrm{~d}, J=5 \mathrm{~Hz}, 1 \mathrm{H}), 8.03(\mathrm{~d}, J=9 \mathrm{~Hz}, 1 \mathrm{H})$, $7.98(\mathrm{~d}, J=2 \mathrm{~Hz}, 1 \mathrm{H}), 7.46(\mathrm{~d}, J=5 \mathrm{~Hz}, 1 \mathrm{H}), 7.38(\mathrm{dd}, J=9 \mathrm{~Hz}, 3 \mathrm{~Hz}, 1 \mathrm{H}), 5.79(\mathrm{~s}, 1 \mathrm{H}), 5.76(\mathrm{~d}, J=7 \mathrm{~Hz}, 1 \mathrm{H})$, $4.78(\mathrm{dd}, J=7 \mathrm{~Hz}, 2 \mathrm{~Hz}, 1 \mathrm{H}), 4.07$ (s, 3H), $3.49(\mathrm{~s}, 3 \mathrm{H}), 1.49$ (s, 9H), 0.92 (t, $J=8 \mathrm{~Hz}, 9 \mathrm{H}), 0.61(\mathrm{t}, J=8 \mathrm{~Hz}, 6 \mathrm{H})$. ${ }^{13} \mathrm{C}$ NMR $\left(\mathrm{CDCl}_{3}, 100 \mathrm{MHz}\right) \delta 169.1,158.5,155.1,147.1,145.2,144.5,131.7,126.6,122.5,119.1,101.5,80.3$, 72.5, 59.5, 56.4, 51.7, 28.5, 6.9, 4.8. IR (NaCl, neat): 3442, 2955, 2877, 1747, 1705, 1622, 1498, 1365, 1239, 1164 , $1112 \mathrm{~cm}^{-1}$. $[\alpha]_{\mathrm{D}}^{25}-22.3\left(\right.$ c $\left.0.69, \mathrm{CHCl}_{3}\right)$. HRMS (FAB+) Calcd for $\mathrm{C}_{25} \mathrm{H}_{39} \mathrm{~N}_{2} \mathrm{O}_{6} \mathrm{Si}$ : 491.2577. Found 491.2583 $(\mathrm{M}+\mathrm{H})$.<smiles>CCOC(NC(C(=O)OC)C(OCC)C(=O)OC)c1ccnc2ccc(OC)cc12</smiles><smiles>COc1ccc2nccc(C)c2c1</smiles>

(2R,3R)-2-tert-Butoxycarbonylamino-3-(6-methoxyquinol-3-yl)-3-triethylsilyloxy-propanal (S-35). To a solution of $11(1.50 \mathrm{~g}, 3.06 \mathrm{mmol})$ in toluene $(30 \mathrm{~mL})$ cooled to $-78^{\circ} \mathrm{C}$ was added DIBAL-H $(12 \mathrm{~mL}, 12 \mathrm{mmol}$, $1.0 \mathrm{M}$ in hexanes), and the mixture was stirred for $20 \mathrm{~min}$. TLC analysis indicated incomplete conversion, so additional DIBAL-H $(8.2 \mathrm{~mL}, 8.2 \mathrm{mmol}, 1.0 \mathrm{M}$ soln in hexanes) was added in two portions after $30 \mathrm{~min}$ and $60 \mathrm{~min}$. After stirring an additional $10 \mathrm{~min}$ the reaction was quenched with methanol $(4 \mathrm{~mL})$ and allowed to warm to rt. To the mixture was added $\mathrm{NH}_{4} \mathrm{Cl}$ aq. Soln $(10 \mathrm{~mL})$ and EtOAc $(100 \mathrm{~mL})$. The organic layer was washed with saturated $\mathrm{NaHCO}_{3}$ aqueous solution $(10 \mathrm{~mL})$, water $(10 \mathrm{~mL})$, and brine $(10 \mathrm{~mL})$. Dried over $\mathrm{Na}_{2} \mathrm{SO}_{4}$ concentrated under reduced pressure. The crude product was subjected to column chromatography on silica gel (eluted with $5: 1$ hexanes/EtOAc) to give $1.14 \mathrm{~g}(2.47 \mathrm{mmol}, 81 \%)$ of $\mathbf{S - 3 5}$ as white solid. Melting point $86-87{ }^{\circ} \mathrm{C} .{ }^{1} \mathrm{H} \mathrm{NMR}\left(\mathrm{CDCl}_{3}\right.$, $400 \mathrm{MHz}) \delta 9.41(\mathrm{~s}, 1 \mathrm{H}), 8.82(\mathrm{~d}, J=5 \mathrm{~Hz}, 1 \mathrm{H}), 8.13(\mathrm{~d}, J=2 \mathrm{~Hz}, 1 \mathrm{H}), 8.07(\mathrm{~d}, J=9 \mathrm{~Hz}, 1 \mathrm{H}), 7.67(\mathrm{~d}, J=5 \mathrm{~Hz}$, $1 \mathrm{H}), 7.42(\mathrm{dd}, J=9 \mathrm{~Hz}, 2 \mathrm{~Hz}, 1 \mathrm{H}), 6.01(\mathrm{~s}, 1 \mathrm{H}), 5.81(\mathrm{~d}, J=5 \mathrm{~Hz}, 1 \mathrm{H}), 4.71(\mathrm{dd}, J=5 \mathrm{~Hz}, 2 \mathrm{~Hz}, 1 \mathrm{H}), 4.09$ (s, 3H), $1.51(\mathrm{~s}, 9 \mathrm{H}), 0.91(\mathrm{t}, J=8 \mathrm{~Hz}, 9 \mathrm{H}), 0.60(\mathrm{q}, J=8 \mathrm{~Hz}, 6 \mathrm{H}) .{ }^{13} \mathrm{C} \mathrm{NMR}\left(\mathrm{CDCl}_{3}, 100 \mathrm{MHz}\right) \delta 196.2,158.9,155.6$, 147.6, 144.8, 144.5, 131.9, 126.6, 123.1, 118.9, 101.8, 80.4, 71.7, 64.3, 56.6, 28.5, 6.9, 4.7. IR (NaCl, neat): 3431 ,

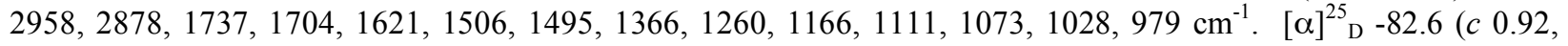
$\mathrm{CH}_{2} \mathrm{Cl}_{2}$ ). HRMS (FAB+) Calcd for $\mathrm{C}_{24} \mathrm{H}_{37} \mathrm{~N}_{2} \mathrm{O}_{5} \mathrm{Si}$ : 461.2472. Found $461.2477(\mathrm{M}+\mathrm{H})$. Anal. Calc. for $\mathrm{C}_{24} \mathrm{H}_{36} \mathrm{~N}_{2} \mathrm{O}_{5} \mathrm{Si}:$ C, 62.58; H, 7.88; N, 6.08. Found: C, 62.15; H, 7.59; N, 6.26.<smiles>CCCC(=O)NC(OCC)C(O[Ga]CC)C(=O)c1ccnc2ccc(S(C)(=O)=O)cc12</smiles>

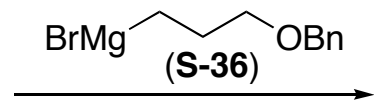

S-35<smiles>CCOC(=O)C(NC(OCC)c1ccnc2ccc(OC)cc12)C(O)CCCOCc1ccccc1</smiles>

12

(1R,2R,3R)-6-Benzyloxy-2-tert-butoxycarbonylamino-3-hydroxy-1-(6-methoxyquinol-3-yl)-1-triethylsilyloxy6-hexane (12). To a suspension of $\mathrm{Mg}(265 \mathrm{mg}, 10.9 \mathrm{mmol})$ and catalytic $\mathrm{I}_{2}$ (ca. $\left.0.1 \mathrm{mg}\right)$ in $\mathrm{Et}_{2} \mathrm{O}(10 \mathrm{~mL})$ was slowly added benzyl 3-bromopropyl ether $(\mathbf{S}-36)^{4}(1.92 \mathrm{~mL}, 10.90 \mathrm{mmol})$ at room temperature. The reaction was stirred for 1 hour, and then added via cannula to a solution of $\mathbf{S}-\mathbf{3 5}(1.00 \mathrm{~g}, 2.17 \mathrm{mmol})$ in THF $(5 \mathrm{~mL})$ at $-78^{\circ} \mathrm{C}$. The resulting pale yellow solution was stirred for an additional $15 \mathrm{~min}$ at $-78{ }^{\circ} \mathrm{C}$. Saturated $\mathrm{NH}_{4} \mathrm{Cl}$ and saturated $\mathrm{NaHCO}_{3}$ solutions were added to the reaction mixture, successively. The aqueous layer was extracted with EtOAc $(3 \times 5 \mathrm{~mL})$. The combined organic layers were washed with brine, dried over $\mathrm{Na}_{2} \mathrm{SO}_{4}$, and concentrated under reduced pressure. The crude oil was purified by flash chromatography on silica gel (eluted with 4:1-1:1

\footnotetext{
${ }^{4}$ For a preparation of 36, see: Bessodes, M.; Boukarim, C. Synlett 1996, 11, 1119-1120.
} 
hexanes/EtOAc solvent gradient) to afford $1.15 \mathrm{~g}(1.88 \mathrm{mmol}, 87 \%)$ of $\mathbf{1 2}$ as colorless oil. ${ }^{1} \mathrm{H} \mathrm{NMR}\left(\mathrm{CDCl}_{3}, 300\right.$ MHz) $\delta 8.78(\mathrm{~d}, J=4 \mathrm{~Hz}, 1 \mathrm{H}), 8.02(\mathrm{~d}, J=9 \mathrm{~Hz}, 1 \mathrm{H}), 7.96(\mathrm{~d}, J=3 \mathrm{~Hz}, 1 \mathrm{H}), 7.60(\mathrm{~d}, J=4 \mathrm{~Hz}, 1 \mathrm{H}), 7.38(\mathrm{dd}, J=$ $9 \mathrm{~Hz}, 3 \mathrm{~Hz}, 1 \mathrm{H}), 7.40-7.15(\mathrm{~m}, 5 \mathrm{H}), 5.94(\mathrm{br} \mathrm{s}, 1 \mathrm{H}), 5.58(\mathrm{~d}, J=8 \mathrm{~Hz}, 1 \mathrm{H}), 4.33(\mathrm{~s}, 2 \mathrm{H}), 4.08(\mathrm{~s}, 3 \mathrm{H}), 3.89(\mathrm{~m}, 1 \mathrm{H})$, 3.87 (br s, 1H), $3.73(\mathrm{br} \mathrm{d}, J=8 \mathrm{~Hz}, 1 \mathrm{H}), 3.28(\mathrm{~m}, 2 \mathrm{H}), 1.51(\mathrm{~s}, 9 \mathrm{H}), 1.65-1.10(\mathrm{~m}, 4 \mathrm{H}), 0.93(\mathrm{t}, J=8 \mathrm{~Hz}, 9 \mathrm{H}), 0.62$ $(\mathrm{q}, J=8 \mathrm{~Hz}, 6 \mathrm{H}) .{ }^{13} \mathrm{C} \mathrm{NMR}\left(\mathrm{CDCl}_{3}, 75 \mathrm{MHz}\right) \delta 158.5,156.1,147.2,144.9,144.4,138.5,131.5,128.4,127.7$, $127.5,126.3,122.8,118.7,101.5,79.8,74.8,72.9,70.1,68.6,56.5,56.2,31.1,28.7,25.8,7.0,4.7$. IR (NaCl, neat): $3504,3446,2956,2877,1701,1622,1507,1489,1365,1241,1168,1102 \mathrm{~cm}^{-1}$. [ $\left.\alpha\right]^{25}-24.4\left(c 0.82, \mathrm{CHCl}_{3}\right)$. HRMS (FAB+) Calcd for $\mathrm{C}_{34} \mathrm{H}_{51} \mathrm{~N}_{2} \mathrm{O}_{6} \mathrm{Si}$ : 611.3516. Found $611.3520(\mathrm{M}+\mathrm{H})$.<smiles>CCOC(=O)C(NC(OCC)C(O)C(C)(C)C)C(O)CCCOc1ccccc1</smiles><smiles>CCOC(=O)C(NC(OCC)c1ccnc2ccc(OC)cc12)C(OCC)C(=O)OCCOCc1ccccc1</smiles>

(1R,2R)-6-Benzyloxy-2-tert-butoxycarbonylamino-3-oxo-1-(6-methoxyquinol-3-yl)-1-triethylsilyloxy-hexane (S-37). To a solution of $12(2.80 \mathrm{~g}, 4.59 \mathrm{mmol})$ in $\mathrm{CH}_{2} \mathrm{Cl}_{2}(45 \mathrm{~mL})$ was added Dess-Martin periodinane $(2.34 \mathrm{~g}$, $5.51 \mathrm{mmol}$ ) at room temperature and the resulting suspension was stirred for 1 hour. Saturated $\mathrm{NaHCO}_{3}$ aqueous solution and $\mathrm{Na}_{2} \mathrm{~S}_{2} \mathrm{O}_{3}$ (s) were added to the reaction mixture, successively. After additional stirring for 30 min, the product was extracted with EtOAc $(3 \times 20 \mathrm{~mL})$. The combined organic layers were washed with brine, dried over $\mathrm{Na}_{2} \mathrm{SO}_{4}$, and concentrated under reduced pressure. The residual oil was purified by silica gel chromatography to afford $2.38 \mathrm{~g}(3.91 \mathrm{mmol}, 85 \%)$ of $\mathbf{S - 3 7}$ as a colorless oil. ${ }^{1} \mathrm{H}$ NMR $\left(\mathrm{CDCl}_{3}, 400 \mathrm{MHz}\right) \delta 8.70(\mathrm{~d}, J=5 \mathrm{~Hz}, 1 \mathrm{H}), 8.12$ (br s, 1H), 8.06 (dd, $J=9 \mathrm{~Hz}, 2 \mathrm{~Hz}, 1 \mathrm{H}), 7.55(\mathrm{~d}, J=4 \mathrm{~Hz}, 1 \mathrm{H}), 7.42(\mathrm{~m}, 1 \mathrm{H}), 7.34-7.25(\mathrm{~m}, 3 \mathrm{H}), 7.19(\mathrm{~d}, J=8 \mathrm{~Hz}$, 2H), $5.89(\mathrm{~m}, 1 \mathrm{H}), 5.79(\mathrm{br} \mathrm{s}, 1 \mathrm{H}), 4.89(\mathrm{~m}, 1 \mathrm{H}), 4.34(\mathrm{~s}, 2 \mathrm{H}), 4.08(\mathrm{~s}, 3 \mathrm{H}), 3.21(\mathrm{~m}, 2 \mathrm{H}), 2.03-1.60(\mathrm{~m}, 4 \mathrm{H}), 1.45(\mathrm{~s}$, $9 \mathrm{H}), 0.91(\mathrm{t}, J=8 \mathrm{~Hz}, 9 \mathrm{H}), 0.60(\mathrm{q}, J=8 \mathrm{~Hz}, 6 \mathrm{H}) .{ }^{13} \mathrm{C} \mathrm{NMR}\left(\mathrm{CDCl}_{3}, 100 \mathrm{MHz}\right) \delta 204.7,158.7,155.2,147.4$, 145.3, 144.7, 138.5, 131.9, 128.6 (2C), 127.7 (2C), 126.6, 122.9, 119.2, 102.0, 80.0, 72.9, 68.9, 64.7, 56.6, 40.2,

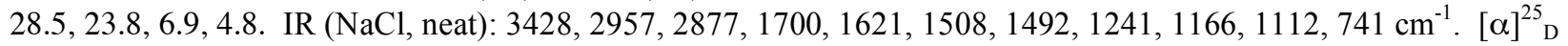
$-83.5\left(c 0.60, \mathrm{CDCl}_{3}\right)$. HRMS (FAB+) Calcd for $\mathrm{C}_{34} \mathrm{H}_{49} \mathrm{~N}_{2} \mathrm{O}_{6} \mathrm{Si}$ : 609.3360. Found $609.3360(\mathrm{M}+\mathrm{H})$.<smiles>CCOC(NC(C(=O)CCCOc1ccccc1)C(OCC)C(=O)OC)c1ccnc2ccc(OC)cc12</smiles>

S-37

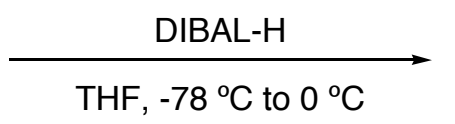

THF, $-78^{\circ} \mathrm{C}$ to $0^{\circ} \mathrm{C}$<smiles>CCOC(N)C(OCC)[C@H](O)CCCOCc1ccccc1</smiles>

15

$(1 R, 2 R, 3 S)$-6-Benzyloxy-2-tert-butoxycarbonylamino-3-hydroxy-1-(6-methoxyquinol-3-yl)-1-triethylsilyloxyhexane (15). To a solution of ketone S-37 $(2.38 \mathrm{~g}, 3.91 \mathrm{mmol})$ in THF $(40 \mathrm{~mL})$ cooled to $-78{ }^{\circ} \mathrm{C}$ was added DIBAL-H $(5.9 \mathrm{~mL}$ of a $1.0 \mathrm{M}$ solution in hexanes, $5.90 \mathrm{mmol})$. The reaction was stirred for 1 hour, then warmed to $0{ }^{\circ} \mathrm{C}$ and stirred for an additional 15 minutes. Saturated $\mathrm{NaHCO}_{3}$ and EtOAc were added to the reaction mixture. The aqueous layer was extracted with EtOAc $(3 \times 10 \mathrm{~mL})$ and the combined organic layers were washed with brine, dried over $\mathrm{Na}_{2} \mathrm{SO}_{4}$, and concentrated under reduced pressure. The residual oil was purified by silica gel chromatography to afford $1.6 \mathrm{~g}(2.62 \mathrm{mmol}, 67 \%)$ of $15 .{ }^{1} \mathrm{H} \mathrm{NMR}\left(\mathrm{CDCl}_{3}, 300 \mathrm{MHz}\right) \delta 8.66(\mathrm{~d}, J=4 \mathrm{~Hz}, 1 \mathrm{H}), 7.95$ $(\mathrm{d}, J=10 \mathrm{~Hz}, 1 \mathrm{H}), 7.90($ br d, $J=2 \mathrm{~Hz}, 1 \mathrm{H}), 7.55(\mathrm{~m}, 1 \mathrm{H}), 7.36-7.24(\mathrm{~m}, 6 \mathrm{H}), 5.79$ (br s, $1 \mathrm{H}), 5.11($ br s, $1 \mathrm{H}), 4.42$ $(\mathrm{s}, 2 \mathrm{H}), 4.18(\mathrm{~m}, 1 \mathrm{H}), 4.08(\mathrm{~s}, 3 \mathrm{H}), 3.60(\mathrm{~m}, 1 \mathrm{H}), 3.52(\mathrm{~m}, 1 \mathrm{H}), 3.36(\mathrm{~m}, 2 \mathrm{H}), 1.45(\mathrm{~s}, 9 \mathrm{H}), 1.80-1.20(\mathrm{~m}, 4 \mathrm{H}), 0.91$ $(\mathrm{t}, J=8 \mathrm{~Hz}, 9 \mathrm{H}), 0.59(\mathrm{q}, J=8 \mathrm{~Hz}, 6 \mathrm{H}) .{ }^{13} \mathrm{C} \mathrm{NMR}\left(\mathrm{CDCl}_{3}, 75 \mathrm{MHz}\right) \delta 158.5,155.8,146.9,145.8,144.3,138.3$, 131.4, 128.5(2C's), 128.5 (2C), 127.7, 126.8, 122.5, 119.3, 101.9, 79.7, 73.1, 72.2, 70.3, 58.6, 56.3, 31.8, 28.6, 26.7, 7.1, 4.9. IR (NaCl, neat): $3446,3274,2955,2876,1704,1622,1509,1496,1365,1242,1170,1110 \mathrm{~cm}^{-1} .[\alpha]_{D}^{25}$ $23.4\left(c 1.1, \mathrm{CHCl}_{3}\right)$. HRMS (FAB+) Calcd for $\mathrm{C}_{34} \mathrm{H}_{51} \mathrm{~N}_{2} \mathrm{O}_{6} \mathrm{Si}$ : 611.3516. Found $611.3527(\mathrm{M}+\mathrm{H})$. 


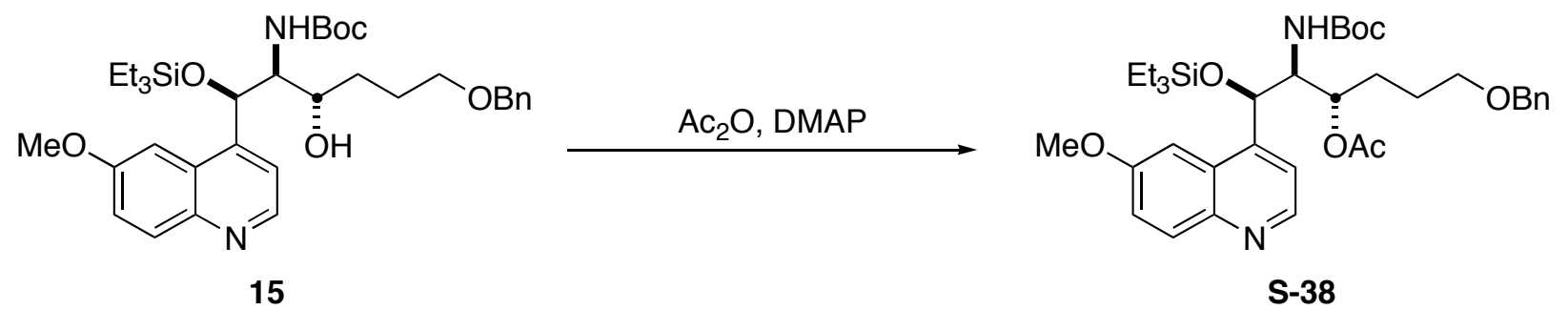

(1R,2S,3S)-6-Benzyloxy-3-acetoxy-2-tert-butoxycarbonylamino-1-(6-methoxyquinol-3-yl)-1-triethylsilyloxyhexane (S-38). To a solution of $15(1.60 \mathrm{~g}, 2.62 \mathrm{mmol})$ and DMAP $(0.96 \mathrm{~g}, 7.86 \mathrm{mmol})$ in $\mathrm{CH}_{2} \mathrm{Cl}_{2}(25 \mathrm{~mL})$ was added acetic anhydride $(495 \mu 1,5.24 \mathrm{mmol})$ at $0{ }^{\circ} \mathrm{C}$, and the mixture was stirred at room temperature for $3 \mathrm{~h}$. EtOAc and saturated $\mathrm{NaHCO}_{3}$ aqueous solution were added to the mixture. The organic layer was separated and washed with brine, dried over $\mathrm{Na}_{2} \mathrm{SO}_{4}$, and concentrated under reduced pressure. The residue was passed through short column on $\mathrm{SiO}_{2}\left(10 \mathrm{~g}\right.$, eluted with EtOAc) to afford $1.70 \mathrm{~g}(2.60 \mathrm{mmol}$, quant. yield $)$ of $\mathbf{S - 3 8}$ as a colorless oil. ${ }^{1} \mathrm{H}$ NMR $\left(\mathrm{CDCl}_{3}, 400 \mathrm{MHz}\right) \delta 8.72(\mathrm{~d}, J=5 \mathrm{~Hz}, 1 \mathrm{H}), 8.02(\mathrm{~d}, J=9 \mathrm{~Hz}, 1 \mathrm{H}), 7.69(\mathrm{~s}, 1 \mathrm{H}), 7.47(\mathrm{~s}, 1 \mathrm{H}), 7.40-7.25(\mathrm{~m}$, $6 \mathrm{H}), 5.53(\mathrm{~s}, 1 \mathrm{H}), 5.07(\mathrm{~s}, 2 \mathrm{H}), 4.46(\mathrm{~s}, 3 \mathrm{H}), 4.06(\mathrm{~s}, 3 \mathrm{H}), 3.39(\mathrm{~s}, 2 \mathrm{H}), 1.50(\mathrm{br} \mathrm{s}, 2 \mathrm{H}), 1.46(\mathrm{~s}, 9 \mathrm{H}), 1.91-1.78(\mathrm{~m}$, $2 \mathrm{H}), 1.35-1.23(\mathrm{~m}, 2 \mathrm{H}), 0.91(\mathrm{t}, J=8 \mathrm{~Hz}, 9 \mathrm{H}), 0.58(\mathrm{q}, J=8 \mathrm{~Hz}, 6 \mathrm{H}) .{ }^{13} \mathrm{C} \mathrm{NMR}\left(\mathrm{CDCl}_{3}, 100 \mathrm{MHz}\right) \delta 170.3,158.3$, 155.6, 147.4, 145.5, 144.4, 138.6, 131.7, 128.5, 127.7, 126.5, 122.4, 118.5, 101.4, 80.0, 73.0, 71.0, 69.9, 56.2(2C:Bn methylene and $\mathrm{OMe}$ ), 28.5, 28.2, 25.3, 20.9, 7.0, 4.8. IR ( $\mathrm{NaCl}$, neat) 2956, 2876, 2359, 2343, 1737, 1701, 1621, $1508,1365,1241,1169,1109,1019,737,478 \mathrm{~cm}^{-1} .[\alpha]^{25}-14.6\left(c 0.92, \mathrm{CDCl}_{3}\right)$. HRMS (FAB+) Calcd for $\mathrm{C}_{36} \mathrm{H}_{53} \mathrm{~N}_{2} \mathrm{O}_{7} \mathrm{Si}:$ 653.3622. Found $653.3644(\mathrm{M}+\mathrm{H})$.<smiles>CCOC(NC(CCCOCc1ccccc1)[C@@H](OCC)OC(C)=O)c1ccnc2ccc(OC)cc12</smiles>

S-38

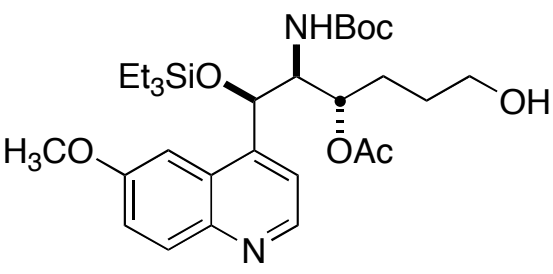

S-39

(1R,2S,3S)-3-acetoxy-2-tert-butoxycarbonylamino-1-(6-methoxyquinol-3-yl)-1-triethylsilyloxy-hexan-6-ol (S39). To a solution of S-38 dissolved in a 1:1 mixture of $\mathrm{MeOH}$ and EtOAc $(10 \mathrm{~mL})$ was added $10 \%$ Pd-C (250 mg), and the mixture was stirred for $24 \mathrm{~h}$ under atmospheric pressure of hydrogen at room temperature. The mixture was filtered through Celite and washed with EtOAc. The filtrate was concentrated under reduced pressure and the residual oil was subjected to column chromatography on silica gel (25 g, eluted with 1:1:0-10:10:1 hexanes/EtOAc/MeOH) to give $386 \mathrm{mg}(82 \%)$ of $\mathbf{S - 3 9}$ as a colorless oil. ${ }^{1} \mathrm{H}$ NMR $\left(\mathrm{CDCl}_{3}, 400 \mathrm{MHz}\right) \delta 8.73(\mathrm{~d}, J=$ $4 \mathrm{~Hz}, 1 \mathrm{H}), 8.02$ (d, $J=9 \mathrm{~Hz}, 1 \mathrm{H}), 7.76($ br s, $1 \mathrm{H}), 7.48$ (br s, 1H), 7.38 (dd, $J=9 \mathrm{~Hz}, 3 \mathrm{~Hz}, 1 \mathrm{H}), 5.52$ (br s, 1H), 5.09 (br s, 2H), 4.49-4.43 (m, 1H), $4.06(\mathrm{~s}, 3 \mathrm{H}), 3.60-3.54(\mathrm{~m}, 2 \mathrm{H}), 1.89-1.79(\mathrm{~m}, 2 \mathrm{H}), 1.47$ (br s, 12H), 1.72-1.15 $(\mathrm{m}, 2 \mathrm{H}), 0.91(\mathrm{t}, J=8 \mathrm{~Hz}, 9 \mathrm{H}), 0.59(\mathrm{q}, J=8 \mathrm{~Hz}, 6 \mathrm{H}) .{ }^{13} \mathrm{C} \mathrm{NMR}\left(\mathrm{CDCl}_{3}, 100 \mathrm{MHz}\right) \delta 170.4,158.3,155.6,147.2$, $145.7,144.2,131.5,126.5,122.4,118.4,101.3,80.0,72.8,70.9,62.3,56.2,56.1,28.4,28.1,27.9,20.8,7.0,4.8$. IR (NaCl, neat): 3278, 2956, 2877, 1736, 1699, 1622, 1509, 1366, 1242, 1169, $1020 \mathrm{~cm}^{-1} .[\alpha]_{\mathrm{D}}^{25}-19.4\left(c 0.95, \mathrm{CHCl}_{3}\right)$. HRMS (FAB+) Calcd for $\mathrm{C}_{29} \mathrm{H}_{47} \mathrm{~N}_{2} \mathrm{O}_{7} \mathrm{Si}: 563.3153$. Found $563.3152(\mathrm{M}+\mathrm{H})$. 
<smiles>CCOC(c1ccnc2ccc(OC)cc12)C(NC(=O)OCc1ccccc1)[C@H](CCCO)OC(C)(C)C</smiles>

S-39

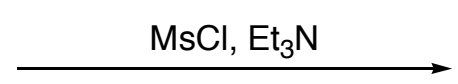<smiles>CCCOC(C(=O)OC)C(NC(OCC)c1ccnc2ccc(OC)cc12)[C@H](CCCOC)OC(C)=O</smiles>

16

(1R,2S,3S)-3-acetoxy-2-tert-butoxycarbonylamino-6-methansulfonyloxy-1-(6-methoxyquinol-3-yl)-1-

triethylsilyloxy-hexane (16). To a solution of $\mathbf{S - 3 9}(1.42 \mathrm{~g}, 2.52 \mathrm{mmol})$ in $\mathrm{CH}_{2} \mathrm{Cl}_{2}(25 \mathrm{~mL})$ were added $\mathrm{Et}_{3} \mathrm{~N}(700$ $\mu \mathrm{L}, 5.00 \mathrm{mmol})$ and methanesulfonyl chloride $(290 \mu \mathrm{L}, 3.80 \mathrm{mmol})$ at room temperature. After stirring for $1 \mathrm{~h}$, saturated $\mathrm{NH}_{4} \mathrm{Cl}$ solution, then $\mathrm{NaHCO}_{3}$ solution, and EtOAc were added to the reaction mixture. The aqueous layer was extracted with EtOAc $(3 \times 5 \mathrm{~mL})$ and the combined organic layers were washed with $\mathrm{H}_{2} \mathrm{O}$ and brine, successively. The organic layer was dried over $\mathrm{Na}_{2} \mathrm{SO}_{4}$, and concentrated under reduced pressure. The residual oil was subjected to column chromatography on silica gel (eluted with 1:1 hexanes/EtOAc) to give $1.52 \mathrm{~g}(2.37 \mathrm{mmol}$, 94\%) of 16 as a colorless oil. ${ }^{1} \mathrm{H}$ NMR $\left(\mathrm{CDCl}_{3}, 400 \mathrm{MHz}\right) \delta 8.75(\mathrm{~d}, J=4 \mathrm{~Hz}, 1 \mathrm{H}), 8.03(\mathrm{~d}, J=9 \mathrm{~Hz}, 1 \mathrm{H}), 7.63(\mathrm{~s}$, 1H), 7.49-7.45 (m, 1H), 7.39 (dd, $J=9 \mathrm{~Hz}, 3 \mathrm{~Hz}, 1 \mathrm{H}), 5.51$ (s, 1H), 5.14-4.97 (m, 2H), 4.53-4.44 (m, 1H), 4.18-4.10 $(\mathrm{m}, 1 \mathrm{H}), 4.06(\mathrm{~s}, 3 \mathrm{H}), 2.98(\mathrm{~s}, 3 \mathrm{H}), 1.96-1.36(\mathrm{~m}, 4 \mathrm{H}), 1.49(\mathrm{br} \mathrm{s}, 12 \mathrm{H}), 0.93(\mathrm{t}, J=8 \mathrm{~Hz}, 9 \mathrm{H}), 0.61(\mathrm{q}, J=8 \mathrm{~Hz}$, $6 \mathrm{H}) .{ }^{13} \mathrm{C}$ NMR $\left(\mathrm{CDCl}_{3}, 100 \mathrm{MHz}\right) \delta 170.4,158.5,155.7,147.4,145.5,144.3,131.7,126.5,122.6,118.4,101.3$, $80.3,72.9,70.1,69.6,56.2,55.9,37.5,28.5,27.6,24.8,20.9,7.0,4.9$. IR (NaCl, neat): $3388,3253,2959,2878$, $1738,1703,1621,1509,1359,1241,1175,1111,1018 \mathrm{~cm}^{-1} .[\alpha]^{25}-15.6\left(c 0.95, \mathrm{CHCl}_{3}\right)$. HRMS (FAB+) Calcd for $\mathrm{C}_{30} \mathrm{H}_{49} \mathrm{~N}_{2} \mathrm{O}_{9} \mathrm{SSi}$ : 641.2928. Found $641.2932(\mathrm{M}+\mathrm{H})$.<smiles>CCOC(c1ccnc2ccc(OC)cc12)C(N)[C@H](OC(C)=O)[C@H](CCCOC)OC(C)=O</smiles>

16<smiles>CCOC(c1ccnc2ccc(OC)cc12)C1[C@@H](OC(C)=O)CCCN1C(=O)OCc1ccccc1</smiles>

17

(2S,3S)-3-Acetoxy-N-tert-butoxycarbonyl-2-[(R)-6-methoxyquinol-3-yl-triethylsilyloxy]methyl-piperidine (17). To a solution of $16(636 \mathrm{mg}, 992 \mu \mathrm{mol})$ in THF $(12 \mathrm{~mL})$ was added $\mathrm{NaH}(400 \mathrm{mg}, 9.90 \mathrm{mmol}, 60 \mathrm{wt} \%$ in mineral oil) at room temperature and the mixture was stirred for $0.5 \mathrm{~h}$. Additional $\mathrm{NaH}(600 \mathrm{mg}, 15.00 \mathrm{mmol})$ was added to the reaction and it was stirred for $1.5 \mathrm{~h}$. The reaction mixture was carefully added to a saturated $\mathrm{NH}_{4} \mathrm{Cl}$ aqueous solution, and then saturated $\mathrm{NaHCO}_{3}$ aqueous solution was added to the mixture. The mixture was extracted with EtOAc. The organic layer was washed with brine, dried over $\mathrm{Na}_{2} \mathrm{SO}_{4}$, and concentrated under reduced pressure. The residue was subjected to column chromatography on silica gel (15 g, eluted with 2:1-1:1 hexanes/EtOAc) to give $535 \mathrm{mg}(982 \mu \mathrm{mol}, 99 \%)$ of $\mathbf{1 7}$ as colorless oil. The compound exists as rotamers that are resolved at room temperature in $\mathrm{CDCl}_{3}$, DMSO-d6, or $\mathrm{CD}_{3} \mathrm{OD}$. Variable temperature NMR was used to confirm the presence of rotamers; the signals began to coalesce at $120{ }^{\circ} \mathrm{C}$ in DMSO-d6 and returned to resolved peaks at ambient temperature. In $\mathrm{CD}_{3} \mathrm{OD}$ the compound is predominantly one rotamer $(80 \%)$ and the NMR data for this rotamer is reported. ${ }^{1} \mathrm{H}$ NMR $\left(\mathrm{CD}_{3} \mathrm{OD}, 300 \mathrm{MHz}, 50^{\circ} \mathrm{C}\right) \delta 8.51(\mathrm{~d}, J=5 \mathrm{~Hz}, 1 \mathrm{H}), 8.19(\mathrm{~d}, J=3 \mathrm{~Hz}, 1 \mathrm{H}), 7.91(\mathrm{~d}, J=9 \mathrm{~Hz}$, $1 \mathrm{H}), 7.37$ (dd, $J=9 \mathrm{~Hz}, 3 \mathrm{~Hz}, 1 \mathrm{H}), 7.30(\mathrm{~d}, J=5 \mathrm{~Hz}, 1 \mathrm{H}), 5.60(\mathrm{q}, J=3 \mathrm{~Hz}, 1 \mathrm{H}), 5.31$ (d, $J=11 \mathrm{~Hz}, 1 \mathrm{H}), 5.20$ (br $\mathrm{d}, J=11 \mathrm{~Hz}, 1 \mathrm{H}), 4.06(\mathrm{~s}, 1 \mathrm{H}), 4.02(\mathrm{~s}, 3 \mathrm{H}), 3.16(\mathrm{td}, J=13 \mathrm{~Hz}, 3 \mathrm{~Hz}, 1 \mathrm{H}), 2.00(\mathrm{~s}, 3 \mathrm{H}), 2.20-1.51(\mathrm{~m}, 4 \mathrm{H}), 0.78(\mathrm{t}$, $J=8 \mathrm{~Hz}, 9 \mathrm{H}), 0.59(\mathrm{~s}, 9 \mathrm{H}), 0.50(\mathrm{q}, J=8 \mathrm{~Hz}, 6 \mathrm{H}) .{ }^{13} \mathrm{C} \mathrm{NMR}\left(\mathrm{CD}_{3} \mathrm{OD}, 100 \mathrm{MHz}\right.$, ambient temperature) $\delta 172.1$, 156.5, 147.7, 147.4, 145.9, 131.4, 129.3, 124.1, 122.6, 105.9, 80.7, 75.1, 69.1, 58.5, 56.4, 39.2, 28.8, 27.9, 24.9, 21.3, 20.7, 7.1, 5.7. IR (NaCl, neat): 2957, 2878, 1738, 1696, 1621, 1508, 1475, 1415, 1365, 1241, 1173, $1105 \mathrm{~cm}^{-1}$. $[\alpha]_{D}^{25}+5.7\left(c 0.68, \mathrm{CHCl}_{3}\right)$. HRMS (FAB+) Calcd for $\mathrm{C}_{29} \mathrm{H}_{45} \mathrm{~N}_{2} \mathrm{O}_{6} \mathrm{Si}$ : 545.3047. Found 545.3066 (M+H). 
<smiles>CCOC(OCC)C1[C@H](OC(C)=O)CCCN1[14C](=O)c1ccccc1</smiles>

17

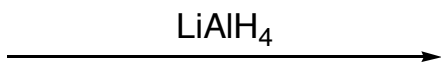<smiles>CCOC(c1ccnc2ccc(OC)cc12)C1[C@H](O)CCCN1C(=O)OCc1ccccc1</smiles>

S-40

(2S,3S)-N-tert-Butoxycarbonyl-3-hydroxy-2-[(R)-6-methoxyquinol-3-yl-triethylsilyloxy]methyl-piperidine (S40). To a $0{ }^{\circ} \mathrm{C}$ solution of $17(599 \mathrm{mg}, 1.13 \mathrm{mmol})$ in $\mathrm{Et}_{2} \mathrm{O}(14 \mathrm{~mL})$ was added $\mathrm{LiAlH}_{4}(215 \mathrm{mg}, 5.66 \mathrm{mmol})$ and the reaction was stirred for 10 minutes. It was quenched carefully with acetone, and then Rochelle's salt solution was added. After stirring for 15 minutes the mixture was diluted with EtOAc and the resulting aqueous layer was extracted with EtOAc $(3 \times 5 \mathrm{~mL})$. The combined organic layers were washed with water, $\mathrm{NaHCO}_{3}$ solution, brine, dried over $\mathrm{Na}_{2} \mathrm{SO}_{4}$, and concentrated. The freezer solid was purified by column chromatography on silica gel (1:1 hexanes/EtOAc) followed by recrystallization from hexanes/ $\mathrm{Et}_{2} \mathrm{O}$ to give $517 \mathrm{mg}(1.06 \mathrm{mmol}, 94 \%)$ of $\mathbf{S}-\mathbf{4 0}$ as a white solid. Melting point $137-138^{\circ} \mathrm{C}$. The compound exists as rotamers that are resolved at ambient temperature and broaden at $120^{\circ} \mathrm{C}$ in DMSO-d6 (also at $50{ }^{\circ} \mathrm{C}$ in $\mathrm{CD}_{3} \mathrm{OD}$ ). ${ }^{1} \mathrm{H}$ NMR $\left(\mathrm{CD}_{3} \mathrm{OD}, 300 \mathrm{MHz}\right.$, ambient temperature) $\delta$ $8.67(\mathrm{~d}, J=4 \mathrm{~Hz}, 0.4 \mathrm{H}), 8.51(\mathrm{~d}, J=4 \mathrm{~Hz}, 0.6 \mathrm{H}), 8.13(\mathrm{~d}, J=3 \mathrm{~Hz}, 0.6 \mathrm{H}), 7.92(\mathrm{~m}, 1 \mathrm{H}), 7.67(\mathrm{~d}, J=5 \mathrm{~Hz}, 0.4$ H), 7.42-7.36 (m, 1H), $7.32(\mathrm{~d}, J=4 \mathrm{~Hz}, 0.6 \mathrm{H}), 5.90(\mathrm{~d}, J=3 \mathrm{~Hz}, 0.4 \mathrm{H}), 5.26(\mathrm{~d}, J=10 \mathrm{~Hz}, 0.6 \mathrm{H}), 4.52(\mathrm{~s}, 0.6$ $\mathrm{H}), 4.38(\mathrm{~s}, 0.6 \mathrm{H}), 4.06(\mathrm{~s}, 1.4 \mathrm{H}), 3.94,4.03-3.94(\mathrm{~m}, 3 \mathrm{H}), 3.15-3.02(\mathrm{~m}, 1 \mathrm{H}), 2.24-1.60(\mathrm{~m}, 4 \mathrm{H}), 1.26(\mathrm{~m}, 2 \mathrm{H})$, 0.96-0.73 (m, 13H), $0.61(\mathrm{~s}, 5 \mathrm{H}), 0.59-0.43(\mathrm{~m}, 6 \mathrm{H}) .{ }^{13} \mathrm{C} \mathrm{NMR}\left(\mathrm{CD}_{3} \mathrm{OD}, 75 \mathrm{MHz}\right) \delta 149.0,147.7,131.4,131.1$, $129.5,124.2,123.4,122.5,121.0,106.6,102.9,81.0,80.6,75.7,74.0,64.6,4.1,62.4,61.9,56.9,56.2,43.8,39.9$, $32.9,29.4,28.9,28.3,27.9,27.2,23.9,19.9,19.7,14.6,7.4,7.2,5.9,5.7$. IR (NaCl, neat): 3254, 2957, 2878, 1676, $1622,1510,1474,1404,1364,1241,1174,1143,1130,1115,1102,731,720 \mathrm{~cm}^{-1} .[\alpha]_{\mathrm{D}}^{25}+32.5(c 0.47, \mathrm{MeOH})$. HRMS (FAB+) Calcd for $\mathrm{C}_{27} \mathrm{H}_{43} \mathrm{~N}_{2} \mathrm{O}_{5} \mathrm{Si}:$ 503.2941. Found $503.2938(\mathrm{M}+\mathrm{H})$. Anal. Calcd for $\mathrm{C}_{27} \mathrm{H}_{42} \mathrm{~N}_{2} \mathrm{O}_{5} \mathrm{Si}$ : C, 64.51; H, 8.42; N, 5.57. Found: C, 64.05; H, 8.13; N, 5.65.

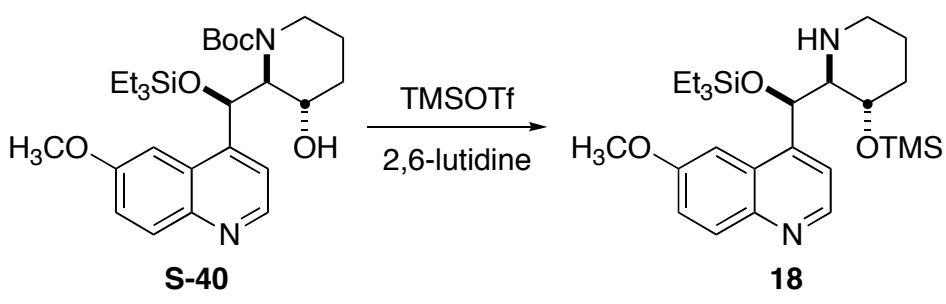

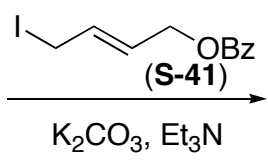<smiles>CCOC(=O)OC/C=C/CN1CCC[C@@H](OC)C1C(OCC)c1ccnc2ccc(OC)cc12</smiles>

(2S,3S)-1-[(E)-4-Benzoyloxy-2-butenyl]-2-[(R)-6-methoxyquinol-3-yl-triethylsilyloxy]methyl-3-

trimethylsilyloxy-piperidine (19). To a solution of $\mathbf{S - 4 0}(205 \mathrm{mg}, 408 \mu \mathrm{mol}) \mathrm{CH}_{2} \mathrm{Cl}_{2}(8 \mathrm{~mL})$ was added 2,6lutidine $\left(230 \mu \mathrm{L}, 1.26 \mathrm{mmol}\right.$, distilled from $\left.\mathrm{CaH}_{2}\right)$ and TMSOTf $(196 \mu \mathrm{L}, 1.68 \mathrm{mmol})$ at room temperature and was stirred for 30 minutes. Additional 2,6-lutidine $(230 \mu \mathrm{L}, 1.26 \mathrm{mmol})$ and TMSOTf $(196 \mu \mathrm{L}, 1.68 \mathrm{mmol})$ were added and the reaction was stirred for 30 minutes. Saturated $\mathrm{NaHCO}_{3}$ aqueous solution and EtOAc were added to the reaction mixture. The separated organic layer was washed with brine, dried over $\mathrm{Na}_{2} \mathrm{SO}_{4}$, and concentrated under reduced pressure to afford crude $\mathbf{1 8}$ (quant) as a colorless oil. An analytical sample was purified column chromatography using neutral silica gel eluted with 1:1:0.01 hexanes/EtOAc/Et $3 \mathrm{~N}$ to afford $\mathbf{1 8}$ as a colorless oil. ${ }^{1} \mathrm{H}$ NMR $\left(\mathrm{CDCl}_{3}, 400 \mathrm{MHz}\right) \delta 8.73(\mathrm{~d}, J=5 \mathrm{~Hz}, 1 \mathrm{H}), 8.02(\mathrm{~d}, J=9 \mathrm{~Hz}, 1 \mathrm{H}), 7.59$ (br s, $\left.1 \mathrm{H}\right), 7.36(\mathrm{dd}, J=9 \mathrm{~Hz}, 3 \mathrm{~Hz}$, $1 \mathrm{H}), 5.62(\mathrm{~d}, J=4 \mathrm{~Hz}, 1 \mathrm{H}), 3.94(\mathrm{~s}, 3 \mathrm{H}), 3.54(\mathrm{ddd}, J=9 \mathrm{~Hz}, 9 \mathrm{~Hz}, 5 \mathrm{~Hz}, 1 \mathrm{H}), 3.06-2.95(\mathrm{~m}, 2 \mathrm{H}), 2.55$ (ddd, $J=13$ $\mathrm{Hz}, 11 \mathrm{~Hz}, 3 \mathrm{~Hz}, 1 \mathrm{H}), 2.02-1.91(\mathrm{~m}, 1 \mathrm{H}), 1.74-1.57(\mathrm{~m}, 2 \mathrm{H}), 1.51-1.15(\mathrm{~m}, 3 \mathrm{H}), 0.88(\mathrm{t}, J=8 \mathrm{~Hz}, 9 \mathrm{H}), 0.57(\mathrm{q}, J=$ $8 \mathrm{~Hz}, 6 \mathrm{H}), 0.03(\mathrm{~s}, 9 \mathrm{H}) .{ }^{13} \mathrm{C} \mathrm{NMR}\left(\mathrm{CDCl}_{3}, 100 \mathrm{MHz}\right) \delta 157.5,147.5,146.8,144.8,131.8,127.7,121.0,120.8$, 103.8, 72.6, 69.2, 68.0, 55.8, 45.7, 34.0, 25.5, 7.0, 5.0, 1.2. HRMS (FAB+) Calcd for $\mathrm{C}_{25} \mathrm{H}_{43} \mathrm{~N}_{2} \mathrm{O}_{5} \mathrm{Si}_{2}: 475.2812$. Found 475.2816 (M+H).

To a solution of crude 18 in DMF $(4 \mathrm{~mL})$ with $\mathrm{K}_{2} \mathrm{CO}_{3}(116 \mathrm{mg}, 840 \mu \mathrm{mol})$ was added $\mathrm{Et}_{3} \mathrm{~N}(117 \mu \mathrm{L}, 840$ $\mu \mathrm{mol})$, and trans-1-iodo-4-benzoyloxy-2-butene (S-41) $(254 \mathrm{mg}, 840 \mu \mathrm{mol})$ at room temperature and the reaction was protected from light. After stirring for $24 \mathrm{~h}, \mathrm{NaHCO}_{3}$ solution, water, and EtOAc were added and the mixture was extracted with EtOAc $(3 \times 10 \mathrm{~mL})$. The combined organic layers were washed saturated $\mathrm{Na}_{2} \mathrm{~S}_{2} \mathrm{O}_{3}$ aqueous 
solution, brine, dried over $\mathrm{Na}_{2} \mathrm{SO}_{4}$, and concentrated under reduced pressure. The residual oil was subjected to column chromatography on neutral silica gel eluted with 5:1:0.01 hexanes/EtOAc/Et $\mathrm{t}_{3} \mathrm{~N}$ to give $246 \mathrm{mg}$ of 19 (379 $\mu \mathrm{mol}, 93 \%)$ as colorless oil. ${ }^{1} \mathrm{H}$ NMR $\left(\mathrm{CDCl}_{3}, 400 \mathrm{MHz}\right) \delta 8.68(\mathrm{~d}, J=4 \mathrm{~Hz}, 1 \mathrm{H}), 8.01-7.97(\mathrm{~m}, 3 \mathrm{H}), 7.66(\mathrm{br}$ s, $1 \mathrm{H}), 7.58-7.53(\mathrm{~m}, 1 \mathrm{H}), 7.44(\mathrm{dd}, J=8 \mathrm{~Hz}, 8 \mathrm{~Hz}, 2 \mathrm{H}), 7.31(\mathrm{dd}, J=9 \mathrm{~Hz}, 2 \mathrm{~Hz}, 1 \mathrm{H}), 7.28(\mathrm{~d}, J=4 \mathrm{~Hz}, 1 \mathrm{H}), 5.41$ $(\mathrm{dt}, J=15 \mathrm{~Hz}, 6 \mathrm{~Hz}, 1 \mathrm{H}), 5.24(\mathrm{~d}, J=9 \mathrm{~Hz}, 1 \mathrm{H}), 4.84-4.75(\mathrm{~m}, 1 \mathrm{H}), 4.44-4.37(\mathrm{~m}, 3 \mathrm{H}), 3.86(\mathrm{~s}, 3 \mathrm{H}), 3.50(\mathrm{dd}, J=$ $14 \mathrm{~Hz}, 7 \mathrm{~Hz}, 1 \mathrm{H}), 3.24$ (d, $J=9 \mathrm{~Hz}, 1 \mathrm{H}), 3.00$ (td, $J=13 \mathrm{~Hz}, 2 \mathrm{~Hz}, 2 \mathrm{H}), 2.56$ (d, $J=14 \mathrm{~Hz}, 1 \mathrm{H}), 2.03$ (qt, $J=13$ $\mathrm{Hz}, 4 \mathrm{~Hz}, 1 \mathrm{H}), 1.92(\mathrm{tt}, J=13 \mathrm{~Hz}, 3 \mathrm{~Hz}, 1 \mathrm{H}), 1.77-1.75(\mathrm{~m}, 2 \mathrm{H}), 1.17-1.10(\mathrm{~m}, 1 \mathrm{H}), 0.81(\mathrm{t}, J=8 \mathrm{~Hz}, 9 \mathrm{H}), 0.46(\mathrm{q}$, $J=8 \mathrm{~Hz}, 6 \mathrm{H}), 0.09(\mathrm{~s}, 9 \mathrm{H}) .{ }^{13} \mathrm{C} \mathrm{NMR}\left(\mathrm{CDCl}_{3}, 100 \mathrm{MHz}\right) \delta 173.4,166.5,157.1,147.3,145.0,134.9,133.0,131.7$, 130.5, 129.8, 128.5, 127.6, 125.3, 121.5, 120.7, 103.6, 77.43, 66.6, 65.9, 65.4, 57.8, 55.6, 47.4, 29.0, 15.3, 6.9, 5.0, 0.2. IR (NaCl, neat): 2953, 2877, 1721, 1621, 1507, 1473, 1452, 1270, 1250, 1094, 1069, 1013, 849, $712 \mathrm{~cm}^{-1}$. $[\alpha]_{D}^{25}-33\left(c 0.70, \mathrm{CHCl}_{3}\right)$. HRMS (FAB+) Calcd for $\mathrm{C}_{36} \mathrm{H}_{53} \mathrm{~N}_{2} \mathrm{O}_{5} \mathrm{Si}_{2}:$ 649.3493. Found 649.3480 (M+H).

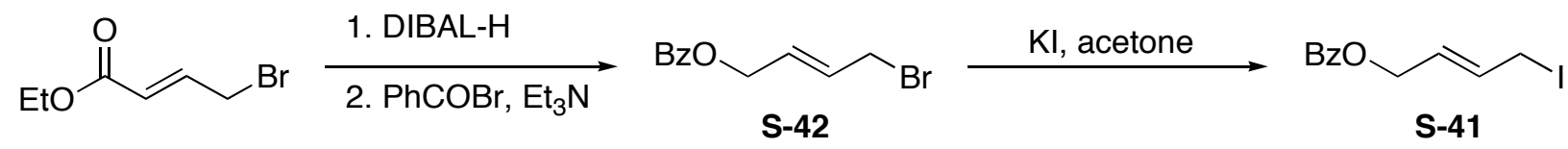

trans-1-Iodo-4-benzoyloxy-2-butene (S-41). To a solution of ethyl bromocrotonate $(5.0 \mathrm{~g}, 25.90 \mathrm{mmol})$ in toluene at $0{ }^{\circ} \mathrm{C}$ was added DIBAL-H $(54.0 \mathrm{~mL}, 54.0 \mathrm{mmol}, 1.0 \mathrm{M}$ in hexanes) dropwise over 1 hour. The reaction was stirred for 1 hour, and then quenched with $2 \mathrm{~N} \mathrm{HCl}$ and diluted with EtOAc. The organic layer was washed with water, brine, dried over $\mathrm{MgSO}_{4}$, and concentrated. The crude oil was purified by column chromatography on silica gel eluted with 4:1 to $1: 1$ hexanes/EtOAc to give $3.51 \mathrm{~g}(23.2 \mathrm{mmol}, 90 \%)$ of trans-4-bromo-2-buten-1-ol ${ }^{5}$ as an oil. To trans-4-bromo-2-buten-1-ol $(3.51 \mathrm{~g}, 23.20 \mathrm{mmol})$ in $\mathrm{Et}_{2} \mathrm{O}(120 \mathrm{~mL})$ at $0{ }^{\circ} \mathrm{C}$ was added triethylamine $(6.50 \mathrm{~mL}$, $46.40 \mathrm{mmol})$ and benzoyl bromide $(4.20 \mathrm{~mL}, 34.80 \mathrm{mmol})$. The thick white mixture was stirred for an additional 30 min, and then $\mathrm{NaHCO}_{3}$ solution was added. The organic layer was diluted with EtOAc, separated and washed with $\mathrm{NaHCO}_{3}$ solution, brine, dried over $\mathrm{MgSO}_{4}$, and concentrated. The crude oil was purified by silica gel chromatography (gradient eluted with $100: 1$ hexanes/Et $3 \mathrm{~N}$ to $100: 2: 1$ hexanes/EtOAc/Et $\left.{ }_{3} \mathrm{~N}\right)$ to give $5.11 \mathrm{~g}(20.0$ mmol, $86 \%$ ) of $\mathbf{S - 4 2}$ as an oil. The spectroscopic properties agreed with those previously reported. To a solution of potassium iodide $(9.96 \mathrm{~g}, 60.00 \mathrm{mmol})$ in acetone $(30 \mathrm{~mL})$ was slowly added trans-4-benzyloxy-1-bromo-2-butene (S-42) $(5.10 \mathrm{~g}, 20.00 \mathrm{mmol})$ as a solution in acetone $(20 \mathrm{~mL})$, and 18-crown-6 $(20 \mathrm{mg}$, catalytic). The reaction mixture was refluxed for 15 hours, cooled to room temperature, and poured into hexanes $(200 \mathrm{~mL})$. The solution was decanted from the solids, and the solids were rinsed with hexanes $(3 \times 25 \mathrm{~mL})$. The combined organic layers were washed with water, $\mathrm{Na}_{2} \mathrm{~S}_{2} \mathrm{O}_{3}$ solution, dried over $\mathrm{MgSO}_{4}$, and concentrated. The crude oil was purified by column chromatography on silica gel (eluted with $25: 1$ hexanes/EtOAc) to give $2.68 \mathrm{~g}(8.87 \mathrm{mmol}, 44 \%)$ of $\mathbf{S}-\mathbf{4 1}$ as a light sensitive red oil. ${ }^{1} \mathrm{H} \mathrm{NMR}\left(\mathrm{CDCl}_{3}, 400 \mathrm{MHz}\right) \oint 8.08-8.04(\mathrm{~m}, 2 \mathrm{H}), 7.58(\mathrm{tt}, J=8 \mathrm{~Hz}, 1 \mathrm{~Hz}, 1 \mathrm{H}), 7.45(\mathrm{t}, J=8$ $\mathrm{Hz}, 2 \mathrm{~Hz}, 2 \mathrm{H}), 6.11(\mathrm{dtt}, J=15 \mathrm{~Hz}, 8 \mathrm{~Hz}, 1 \mathrm{~Hz}, 1 \mathrm{H}), 5.92(\mathrm{dtt}, J=15 \mathrm{~Hz}, 6 \mathrm{~Hz}, 1 \mathrm{~Hz}, 1 \mathrm{H}), 4.82($ br d, $J=6 \mathrm{~Hz}$, 2H), 3.90 (br d, $J=8 \mathrm{~Hz}, 2 \mathrm{H}) .{ }^{13} \mathrm{C} \mathrm{NMR}\left(\mathrm{CDCl}_{3}, 100 \mathrm{MHz}\right) \delta 166.3,133.3,132.0,130.2,129.8,128.6,127.7,64.2$, 4.0. IR (NaCl, neat) 3062, 3030, 2945, 1717, 1600, 1449, 1376, 1267, 1152, 1108, 1070, 1025, 965, $709 \mathrm{~cm}^{-1}$. HRMS (FAB+) Calcd for $\mathrm{C}_{11} \mathrm{H}_{12} \mathrm{IO}_{2}: 302.9882$. Found $302.9891(\mathrm{M}+\mathrm{H})$.

5 (a) Talekar, D. G.; Joshi, P. L.; Ramaiah, P.; Rao, A. S. Indian J. Chem. 1986, 25B, 145-151. (b) Ashton, W. T.; Meurer, L. C.; Cantone, C. L.; Field, A. K.; Hannah, J.; Karkas, J. D.; Liou, R.; Patel, G. F.; Perry, H. C.; Wagner, A. F.; Walton, E.; Tolman, R. L. J. Med. Chem. 1988, 31, 2304-2315. 


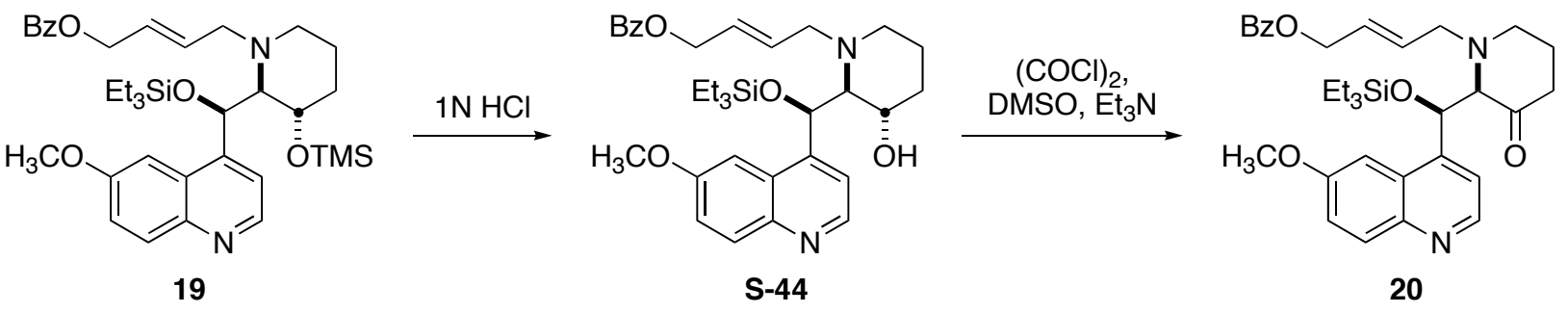

(2R)-1-[(E)-4-Benzolyoxy-2-butenyl]-2-[(R)-6-methoxyquinol-3-yl-triethylsilyloxy]methyl-3-Oxo- piperidine (20). To a solution of $19(200 \mathrm{mg}, 0.31 \mathrm{mmol})$ in THF $(4.5 \mathrm{~mL})$ was added $1 \mathrm{~N} \mathrm{HCl}(0.5 \mathrm{~mL})$ at $0{ }^{\circ} \mathrm{C}$. After stirring for 1 hour, $\mathrm{NaCl}(\mathrm{s})$ was added and the layers were separated. The aqueous layer was extracted with EtOAc $(4 \times 5$ $\mathrm{mL}$ ). The combined organic layers were washed with saturated $\mathrm{NaHCO}_{3}$, brine, dried over $\mathrm{Na}_{2} \mathrm{SO}_{4}$, and concentrated under reduced pressure. The crude oil was very quickly filtered through a small plug of neutral silica gel eluted with 1:1 hexanes/EtOAc to provide $176 \mathrm{mg}$ ( $0.31 \mathrm{mmol}$, quant.) of $\mathbf{S - 4 4}$ as an oil and used immediately in the next reaction. A sample was sacrificed for analysis. ${ }^{1} \mathrm{H} \mathrm{NMR}\left(\mathrm{CDCl}_{3}, 400 \mathrm{MHz}\right) \delta 8.71(\mathrm{~d}, J=5 \mathrm{~Hz}, 1 \mathrm{H}), 8.03-$ $7.97(\mathrm{~m}, 3 \mathrm{H}), 7.64(\mathrm{br} \mathrm{s}, 1 \mathrm{H}), 7.57-7.52(\mathrm{~m}, 1 \mathrm{H}), 7.45-7.31(\mathrm{~m}, 4 \mathrm{H}), 5.53(\mathrm{br} \mathrm{s}, 1 \mathrm{H}), 5.45-5.28(\mathrm{~m}, 2 \mathrm{H}), 4.60(\mathrm{~d}, J=$ $5 \mathrm{~Hz}, 2 \mathrm{H}), 4.23$ (br s, 1H), 3.91 (s, 3H), 3.34 (br s, 1H), 3.12 (br d, $J=6 \mathrm{~Hz}, 1 \mathrm{H}), 2.97$ (td, $J=12 \mathrm{~Hz}, 3 \mathrm{~Hz}, 1 \mathrm{H})$, $2.72(\mathrm{br} \mathrm{s}, 1 \mathrm{H}), 2.51(\mathrm{dt}, J=12 \mathrm{~Hz}, 4 \mathrm{~Hz}, 1 \mathrm{H}), 2.01-1.93(\mathrm{~m}, 1 \mathrm{H}), 1.88-1.67(\mathrm{~m}, 2 \mathrm{H}), 1.59-1.51(\mathrm{~m}, 1 \mathrm{H}), 0.80(\mathrm{t}, J=$ $8 \mathrm{~Hz}, 9 \mathrm{H}), 0.46(\mathrm{q}, J=8 \mathrm{~Hz}, 6 \mathrm{H}) .{ }^{13} \mathrm{C}$ NMR $\left(\mathrm{CDCl}_{3}, 100 \mathrm{MHz}\right) \delta 166.4,157.6,147.4,145.1,133.3,133.1,133.0$, 132.0, 130.3, 129.8, 128.5, 126.1, 121.8, 120.8, 103.2, 68.9, 65.7, 64.9, 60.7, 56.0, 55.7, 45.6, 30.5, 27.4, 19.5, 6.9, 5.0. IR ( NaCl, neat): $3293,2945,2879,1719,1621,1510,1453,1270,1102,1026,858,715 \mathrm{~cm}^{-1} .[\alpha]_{\mathrm{D}}-4.5(c$ 2.12, $\mathrm{CHCl}_{3}$ ). HRMS (FAB+) Calcd for $\mathrm{C}_{33} \mathrm{H}_{45} \mathrm{~N}_{2} \mathrm{O}_{5} \mathrm{Si}$ : 577.3098. Found 577.3107 (M+H).

To a solution of oxalyl chloride $(46 \mu \mathrm{L}, 0.53 \mathrm{mmol})$ in $\mathrm{CH}_{2} \mathrm{Cl}_{2}(2 \mathrm{~mL})$ was added DMSO (56 $\mu \mathrm{L}, 0.79$ mmol) at $-78{ }^{\circ} \mathrm{C}$ and stirred for 25 minutes. To this was added $\mathbf{S}-44(151 \mathrm{mg}, 0.26 \mathrm{mmol})$ as a solution in $\mathrm{CH}_{2} \mathrm{Cl}_{2}(2$ $\mathrm{mL})$ and stirred an additional 45 minutes, then $\mathrm{Et}_{3} \mathrm{~N}(74 \mu \mathrm{L}, 1.58 \mathrm{mmol})$ was added to the reaction mixture. After stirring for an additional 10 minutes, the mixture was allowed to warm to room temperature, and saturated $\mathrm{NaHCO}_{3}$ aqueous solution and EtOAc were added. The aqueous layer was extracted with EtOAc $(3 \times 5 \mathrm{~mL})$. The combined organic layer was washed with brine, dried over $\mathrm{Na}_{2} \mathrm{SO}_{4}$, and concentrated under reduced pressure. The residual oil was subjected to column chromatography on neutral silica gel (eluted with $1: 1$ hexanes/Et $2 \mathrm{O}$ ) to give $134 \mathrm{mg}$ of $\mathbf{2 0}$ $(0.23 \mathrm{mmol}, 90 \%)$ as colorless oil that was carried on immediately. ${ }^{1} \mathrm{H}$ NMR $\left(\mathrm{CDCl}_{3}, 400 \mathrm{MHz}\right) \delta 8.71(\mathrm{~d}, J=5 \mathrm{~Hz}$, $1 \mathrm{H}), 8.00(\mathrm{~d}, J=9 \mathrm{~Hz}, 1 \mathrm{H}), 7.98(\mathrm{dd}, J=7 \mathrm{~Hz}, 1.5 \mathrm{~Hz}, 2 \mathrm{H}), 7.55(\mathrm{tt}, J=7 \mathrm{~Hz}, 1.5 \mathrm{~Hz}, 1 \mathrm{H}), 7.50(\mathrm{br} \mathrm{s}, 1 \mathrm{H}), 7.42(\mathrm{t}$, $J=7 \mathrm{~Hz}, 2 \mathrm{H}), 7.36-7.32(\mathrm{~m}, 1 \mathrm{H}), 7.32(\mathrm{~d}, J=3 \mathrm{~Hz}, 1 \mathrm{H}), 5.63(\mathrm{~d}, J=6 \mathrm{~Hz}, 1 \mathrm{H}), 5.53(\mathrm{dt}, J=16 \mathrm{~Hz}, 6 \mathrm{~Hz}, 1 \mathrm{H})$, $5.33(\mathrm{br} \mathrm{s}, 1 \mathrm{H}), 4.50(\mathrm{~s}, 2 \mathrm{H}), 3.93(\mathrm{~s}, 3 \mathrm{H}), 3.59(\mathrm{~d}, J=5 \mathrm{~Hz}, 1 \mathrm{H}), 3.39(\mathrm{ddd}, J=14 \mathrm{~Hz}, 11 \mathrm{~Hz}, 7 \mathrm{~Hz}, 1 \mathrm{H}), 3.16$ $(\mathrm{ABX}, J=14 \mathrm{~Hz}, 6 \mathrm{~Hz}, \Delta v=40 \mathrm{~Hz}, 2 \mathrm{H}), 2.71(\mathrm{dt}, J=13 \mathrm{~Hz}, 4 \mathrm{~Hz}, 1 \mathrm{H}), 2.65$ (ddd, $J=15 \mathrm{~Hz}, 11 \mathrm{~Hz}, 7 \mathrm{~Hz}, 1 \mathrm{H}), 2.46$ $(\mathrm{dt}, J=15 \mathrm{~Hz}, 5 \mathrm{~Hz}, 1 \mathrm{H}), 2.20-1.85(\mathrm{~m}, 3 \mathrm{H}), 1.25(\mathrm{br} \mathrm{s}, 1 \mathrm{H}), 0.81(\mathrm{t}, J=8 \mathrm{~Hz}, 9 \mathrm{H}), 0.47(\mathrm{q}, J=8 \mathrm{~Hz}, 6 \mathrm{H}) .{ }^{13} \mathrm{C}$ $\operatorname{NMR}\left(\mathrm{CDCl}_{3}, 100 \mathrm{MHz}\right) \delta 209.2,166.3,157.6,147.3,146.1,144.9,133.1,131.9,131.4,130.2,129.8,128.5,127.3$, 126.6, 121.5, 120.6, 102.8, 74.9, 73.4, 64.6, 56.6, 55.7, 46.6, 39.6, 24.2, 6.9 (3C), 5.2 (3C). IR (NaCl, neat) 2954, $2876,1719,1620,1507,1473,1452,1360,1314,1270,1241,1109,1071,1026,978,855,713 \mathrm{~cm}^{-1} .[\alpha]_{\mathrm{D}}^{25}-32(c$ 0.60, $\mathrm{CHCl}_{3}$ ). HRMS (FAB+) Calcd for $\mathrm{C}_{33} \mathrm{H}_{43} \mathrm{~N}_{2} \mathrm{O}_{5} \mathrm{Si}$ : 575.2941. Found 575.2934 (M+H).

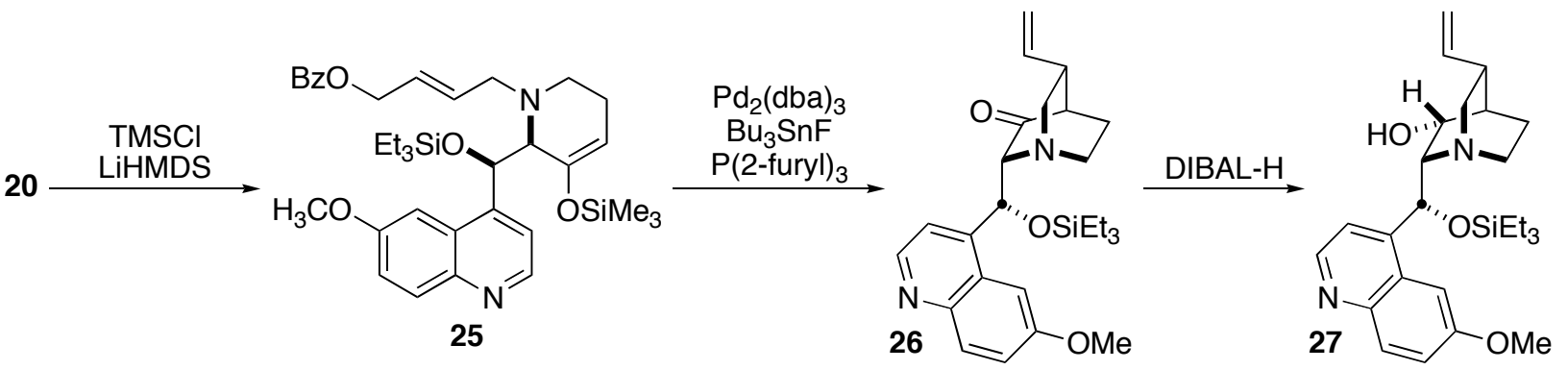

$(2 R)-1-[(E)-4-B e n z o l y o x y-2-b u t e n y l]-2-[(R)-6$-methoxyquinol-3-yl-triethylsilyloxy]methyl-3-trimethylsilyloxy1,2,5,6-tetrahydro-pyridine (27). To a solution of $20(87 \mathrm{mg}, 0.151 \mathrm{mmol})$ in THF $(1 \mathrm{~mL})$ at $-78{ }^{\circ} \mathrm{C}$ was added chlorotrimethylsilane $\left(38 \mu \mathrm{L}, 0.30 \mathrm{mmol}\right.$, freshly distilled from $\left.\mathrm{CaH}_{2}\right)$ and LiHMDS (303 $\mu \mathrm{L}, 0.30 \mathrm{mmol}, 1.0 \mathrm{M}$ solution in THF) and the reaction was stirred for 30 minutes, then warmed to $0{ }^{\circ} \mathrm{C}$ and concentrated under reduced 
pressure. The crude material was quickly purified by column chromatography twice using neutral silica gel eluted with 9:1:0.01 hexanes/EtOAc/Et $3 \mathrm{~N}$ to give $58 \mathrm{mg}(89.3 \mu \mathrm{mol}, 59 \%)$ of $\mathbf{2 5}$. Enol ether $\mathbf{2 5}$ was dried by solvent exchanging with dry toluene and used immediately after purification. ${ }^{1} \mathrm{H} N \mathrm{NMR}\left(\mathrm{CDCl}_{3}, 300 \mathrm{MHz}\right) \delta 8.71(\mathrm{~d}, 5 \mathrm{~Hz}$, $1 \mathrm{H}), 8.05(\mathrm{~d}, J=8 \mathrm{~Hz}, 2 \mathrm{H}), 7.96(\mathrm{~d}, J=9 \mathrm{~Hz}, 1 \mathrm{H}), 7.67-7.52(\mathrm{~m}, 3 \mathrm{H}), 7.48-7.40(\mathrm{~m}, 2 \mathrm{H}), 7.30(\mathrm{dd}, J=9 \mathrm{~Hz}, 3 \mathrm{~Hz}$, 1H), $5.88(\mathrm{ABX}, J=15 \mathrm{~Hz}, 6 \mathrm{~Hz}, 6 \mathrm{~Hz}, \Delta v=10 \mathrm{~Hz}, 2 \mathrm{H}), 5.72(\mathrm{~m}, 1 \mathrm{H}), 4.92(\mathrm{dd}, J=4 \mathrm{~Hz}, 4 \mathrm{~Hz}, 1 \mathrm{H}), 4.83-4.72(\mathrm{~m}$, 2H), 3.92 (s, 3H), 3.40 (dd, $J=14 \mathrm{~Hz}, 6 \mathrm{~Hz}, 1 \mathrm{H}), 3.31$ (s, 1H), 3.22 (dd, $J=14 \mathrm{~Hz}, 5 \mathrm{~Hz}, 1 \mathrm{H}), 2.30-1.89$ (m, 3H), $1.42-1.28(\mathrm{~m}, 1 \mathrm{H}), 0.87(\mathrm{t}, J=8 \mathrm{~Hz}, 9 \mathrm{H}), 0.57-0.49(\mathrm{~m}, 6 \mathrm{H}), 0.18$ (s, 9H).

To a $10 \mathrm{~mL}$ sealed tube containing $\mathrm{n}-\mathrm{Bu}_{3} \mathrm{SnF}$ polymer $(40.00 \mathrm{mg}, 0.13 \mathrm{mmol})$ under argon was added toluene $(1 \mathrm{~mL})$, followed by a solution of $25(58 \mathrm{mg}, 89.3 \mu \mathrm{mmol})$ in toluene $(4 \mathrm{~mL})$, and then a green solution of $\mathrm{Pd}_{2}(\mathrm{dba})_{3}(16.4 \mathrm{mg}, 17.9 \mu \mathrm{mol}, 20 \mathrm{~mol} \%)$ and $\mathrm{P}(2 \text {-furyl })_{3}(16.6 \mathrm{mg}, 71.4 \mu \mathrm{mol})$ in toluene $(4 \mathrm{~mL})$ in rapid succession. The tube was sealed and quickly placed on a pre-heated $85{ }^{\circ} \mathrm{C}$ oil bath and stirred vigorously for 30 minutes. The reaction mixture was removed from the oil bath and placed in a $0{ }^{\circ} \mathrm{C}$ ice-water bath and quenched with $\mathrm{NaHCO}_{3}$ saturated solution $(3 \mathrm{~mL})$. After vigorous stirring for 30 seconds, the layers were separated. The aqueous layer was extracted with EtOAc $(5 \mathrm{~mL})$. The combined organic layers were diluted with additional EtOAc, washed with $\mathrm{NaHCO}_{3}$ solution $(4 \mathrm{~mL})$, and filtered through Florosil resin eluted with EtOAc. The resulting solution was partially concentrated on a rotary evaporator until $4 \mathrm{~mL}$ of solution remained. This was quickly purified by column chromatography using florosil resin eluted with a solvent gradient (1:1 hexanes/EtOAc to EtOAc). The mixed fractions were further purified by column chromatography using neutral silica gel eluted with a solvent gradient ( $1: 1$ hexanes/EtOAc to EtOAc) to give 26. Ketone product $\mathbf{2 6}$ is not stable and was immediately reduced to alcohol 27. ${ }^{1} \mathrm{H}$ NMR $\left(\mathrm{CDCl}_{3}, 400 \mathrm{MHz}\right) \delta 8.83(\mathrm{~d}, J=4 \mathrm{~Hz}, 1 \mathrm{H}), 8.06(\mathrm{~d}, J=9 \mathrm{~Hz}, 1 \mathrm{H}), 7.77(\mathrm{br} \mathrm{s}, 1 \mathrm{H}), 7.38(\mathrm{dd}, J=9 \mathrm{~Hz}, 3$ $\mathrm{Hz}, 1 \mathrm{H}), 7.09$ (br s, 1H), $5.79(\mathrm{~s}, 1 \mathrm{H}), 5.54-5.43(\mathrm{~m}, 1 \mathrm{H}), 4.98-4.87(\mathrm{~m}, 2 \mathrm{H}), 3.95(\mathrm{~m}, 3 \mathrm{H}), 3.42-3.29(\mathrm{~m}, 2 \mathrm{H}), 3.27$ (s, 1H), 2.89-2.73 (m, 2H), 2.60 (br d, $J=14 \mathrm{~Hz}, 1 \mathrm{H}), 2.40(\mathrm{~s}, 1 \mathrm{H}), 2.20-1.87(\mathrm{~m}, 2 \mathrm{H}), 0.89(\mathrm{t}, J=8 \mathrm{~Hz}, 9 \mathrm{H}), 0.62-$ $0.51(\mathrm{~m}, 6 \mathrm{H}) .{ }^{13} \mathrm{C}$ NMR $\left(\mathrm{CDCl}_{3}, 100 \mathrm{MHz}\right) \delta 158.2,147.9,147.1,144.4,140.1,132.3,125.8,121.5,120.0,115.3$, 100.5, 75.1, 72.6, 56.7, 55.9, 46.8, 42.9, 29.9, 25.3, 7.0, 5.1. MS (FAB+) Calcd for $\mathrm{C}_{26} \mathrm{H}_{37} \mathrm{~N}_{2} \mathrm{O}_{3} \mathrm{Si}$ : 453.26. Found $453.27(\mathrm{M}+\mathrm{H})$.

To a $-78{ }^{\circ} \mathrm{C}$ solution of ketone 26 in THF was added DIBAL-H $(0.5 \mathrm{~mL}, 0.50 \mathrm{mmol}, 1.0 \mathrm{M}$ solution in hexanes). The reaction was stirred for 10 minutes, and then warmed to $0{ }^{\circ} \mathrm{C}$ and stirred for 20 minutes. Rochelle salt solution $(3 \mathrm{~mL})$ was added to the reaction mixture and it was stirred an additional 15 minutes. After dilution with EtOAc, the layers were separated and the aqueous layer was extracted with EtOAc. The combined organic layers were washed with $\mathrm{NaHCO}_{3}$ solution, dried over $\mathrm{Na}_{2} \mathrm{SO}_{4}$, filtered and concentrated. The crude oil was purified using combinations of preparative TLC $(9: 1 \mathrm{EtOAc} / \mathrm{MeOH})$ and column chromatography using neutral silica gel eluted with hexanes/EtOAc, EtOAc, and/or EtOAc/MeOH. The desired compound 27 was isolated in 39\% yield over two steps $(16 \mathrm{mg}, 35.2 \mu \mathrm{mol})$ exists as a mixture of rotamers, confirmed by VT-NMR. The two major conformations were determined by rOe NMR to be rotamers about $\mathrm{C} 9 .{ }^{1} \mathrm{H}$ NMR $\left(\mathrm{CDCl}_{3}, 500 \mathrm{MHz}\right.$, ambient temperature) $\delta 8.80(\mathrm{~d}, J=5 \mathrm{~Hz}, 0.6 \mathrm{H}), 8.69(\mathrm{~d}, J=5 \mathrm{~Hz}, 0.3 \mathrm{H}), 8.04(\mathrm{t}, J=9 \mathrm{~Hz}, 1 \mathrm{H}), 7.85(\mathrm{~d}, J=3 \mathrm{~Hz}, 0.3 \mathrm{H})$, $7.63(\mathrm{~d}, J=5 \mathrm{~Hz}, 0.6 \mathrm{H}), 7.48(\mathrm{~s}, 0.2 \mathrm{H}), 7.39$ (dd, $J=9 \mathrm{~Hz}, 2 \mathrm{~Hz}, 0.6 \mathrm{H}), 7.36$ (dd, $J=9 \mathrm{~Hz}, 2 \mathrm{~Hz}, 0.4 \mathrm{H}), 7.16(\mathrm{~d}, J$ $=4 \mathrm{~Hz}, 0.4 \mathrm{H}), 7.06(\mathrm{~s}, 0.2 \mathrm{H}), 5.94(\mathrm{~d}, J=8 \mathrm{~Hz}, 1 \mathrm{H}), 5.91-5.79(\mathrm{~m}, 0.5 \mathrm{H}), 5.35(\mathrm{~d}, J=11 \mathrm{~Hz}, 1 \mathrm{H}), 5.05(\mathrm{~d}, J=17$ $\mathrm{Hz}, 1 \mathrm{H}), 5.03(\mathrm{~d}, J=15 \mathrm{~Hz}, 1 \mathrm{H}), 4.47(\mathrm{~m}, 0.3 \mathrm{H}), 4.33(\mathrm{~m}, 0.6 \mathrm{H}), 3.96(\mathrm{~s}, 2 \mathrm{H}), 3.92(\mathrm{~s}, 1 \mathrm{H}), 3.70-3.62(\mathrm{~m}, 1.2 \mathrm{H})$, 3.38-3.26 (m, 1H), 3.17-3.11 (m, 0.3H), $3.08(\mathrm{t}, J=8 \mathrm{~Hz}, 0.6 \mathrm{H}), 2.97(\mathrm{~s}, 0.4 \mathrm{H}), 2.91-2.85(\mathrm{~m}, 0.7 \mathrm{H}), 2.80-2.74(\mathrm{~m}$, $0.3 \mathrm{H}), 2.67-2.60(\mathrm{~m}, 0.8 \mathrm{H}), 2.56-2.38(\mathrm{~m}, 2.1 \mathrm{H}), 2.34-2.29(\mathrm{~m}, 0.6 \mathrm{H}), 2.25-2.15(\mathrm{~m}, 2 \mathrm{H}), 2.08-2.00(\mathrm{~m}, 0.9 \mathrm{H}), 0.97-$ $0.85(\mathrm{~m}, 1.8 \mathrm{H}), 0.84(\mathrm{t}, J=8 \mathrm{~Hz}, 6 \mathrm{H}), 0.78(\mathrm{t}, J=8 \mathrm{~Hz}, 3 \mathrm{H}), 0.45-0.33(\mathrm{~m}, 6 \mathrm{H})$. IR (NaCl, neat) 3847, 3740, 3613, $3284,2932,1692,1630,1512,1465,1238,1089,912,852,736 \mathrm{~cm}^{-1} .[\alpha]^{25}-4.5\left(c 0.33, \mathrm{CHCl}_{3}\right)$. HRMS (FAB + ) Calcd for $\mathrm{C}_{26} \mathrm{H}_{39} \mathrm{~N}_{2} \mathrm{O}_{3} \mathrm{Si}$ : 455.2730. Found $455.2725(\mathrm{M}+\mathrm{H})$. 

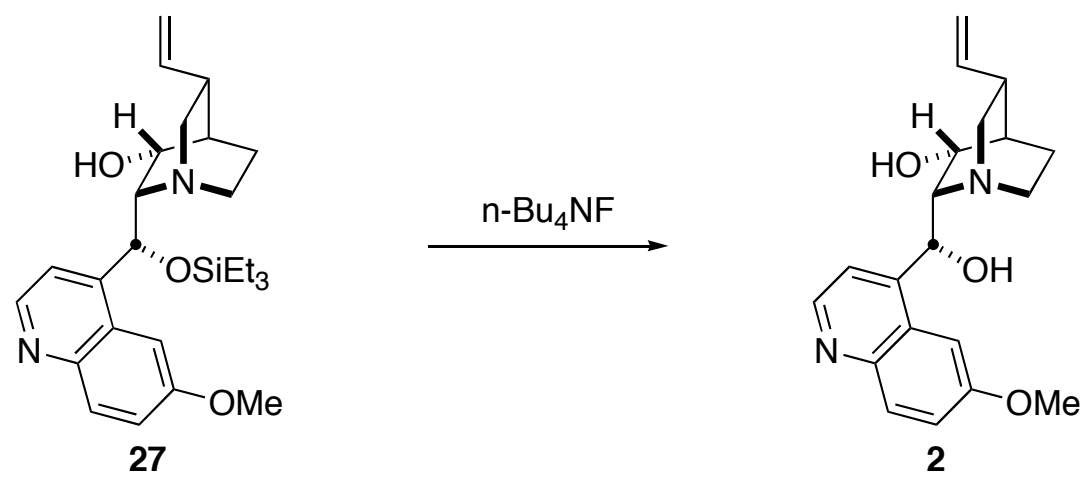

(R)-7-Hydroxy-quinine (2). To a $-45^{\circ} \mathrm{C}$ solution of $27(4.6 \mathrm{mg}, 10.1 \mu \mathrm{mol})$ in THF $(4 \mathrm{~mL})$ was added $\mathrm{n}-\mathrm{Bu}_{4} \mathrm{NF}$ $(30 \mu \mathrm{L}, 30 \mu \mathrm{mmol}, 1.0 \mathrm{M}$ solution in THF) dropwise. The reaction was stirred for 10 minutes, quenched with $\mathrm{NH}_{4} \mathrm{Cl}$ solution $(0.75 \mathrm{~mL})$, and allowed to warm to room temperature. The mixture was diluted with EtOAc and the aqueous layer was extracted with EtOAc $(5 \times 1 \mathrm{~mL})$. The combined organic layers were washed with $\mathrm{NaHCO}_{3}$ solution, dried over $\mathrm{Na}_{2} \mathrm{SO}_{4}$, and concentrated. The crude material was purified by chromatography on neutral silica gel (9:1 EtOAc/MeOH) followed by preparative TLC $(9: 1 \mathrm{EtOAc} / \mathrm{MeOH})$ to give $3 \mathrm{mg}(8.8 \mu \mathrm{mol}, 88 \%)$ of $2 .{ }^{1} \mathrm{H}$ $\operatorname{NMR}\left(\mathrm{CDCl}_{3}, 400 \mathrm{MHz}\right) \delta 8.76(\mathrm{~d}, J=5 \mathrm{~Hz}, 1 \mathrm{H}), 8.02(\mathrm{~d}, J=9 \mathrm{~Hz}, 1 \mathrm{H}), 7.49-7.45(\mathrm{~m}, 2 \mathrm{H}), 7.37(\mathrm{dd}, J=9 \mathrm{~Hz}, 3$ $\mathrm{Hz}, 1 \mathrm{H}), 5.88(\mathrm{ddd}, J=17 \mathrm{~Hz}, 11 \mathrm{~Hz}, 7 \mathrm{~Hz}, 1 \mathrm{H}), 5.79(\mathrm{~d}, J=9 \mathrm{~Hz}, 1 \mathrm{H}), 5.11(\mathrm{br} \mathrm{d}, J=8 \mathrm{~Hz}, 1 \mathrm{H}), 5.07(\mathrm{~d}, J=1$ $\mathrm{Hz}, 1 \mathrm{H}), 4.47$ (dd, $J=7 \mathrm{~Hz}, 4 \mathrm{~Hz}, 1 \mathrm{H}), 3.96(\mathrm{~s}, 3 \mathrm{H}), 3.39-3.14(\mathrm{~m}, 2 \mathrm{H}), 2.99-2.88$ (m, 2H), 2.69-2.46 (m, 3H), 2.26$2.16(\mathrm{~m}, 2 \mathrm{H}) .{ }^{13} \mathrm{C}$ NMR (from HSQC and HMBC NMR, $\left.\mathrm{CDCl}_{3}, 500 \mathrm{MHz}\right) \delta 158.1,148.6,145.7,144.9,141.2$, $132.2,128.1,121.8,119.7,115.8,102.1,69.3,66.7,64.4,55.9,55.4,42.8,40.5,34.8,20.5$. IR (NaCl, neat) 3300 , $2922,2859,1734,1460,1374,1243,1175,1088,1026 \mathrm{~cm}^{-1} .[\alpha]_{\mathrm{D}}^{25}-22\left(c 0.10, \mathrm{CHCl}_{3}\right)$. HRMS (FAB+) Calcd for $\mathrm{C}_{20} \mathrm{H}_{25} \mathrm{~N}_{2} \mathrm{O}_{3}: 341.1865$. Found $341.1868(\mathrm{M}+\mathrm{H})$.<smiles>C=CC1CCCN1C(=O)Cc1ccnc2ccc(OC)cc12</smiles>

29

8-Dehydro-7-keto-quinine (29). Isolated as a byproduct from the Pd-mediated cyclization. It was also prepared by treating ketone 24 with $\mathrm{K}_{2} \mathrm{CO}_{3}$ in THF. ${ }^{1} \mathrm{H}$ NMR $\left(\mathrm{CDCl}_{3}, 500 \mathrm{MHz}\right) \delta 8.76(\mathrm{~d}, J=3 \mathrm{~Hz}, 1 \mathrm{H}), 8.05(\mathrm{~d}, J=9 \mathrm{~Hz}$, $1 \mathrm{H}), 7.51(\mathrm{~m}, 1 \mathrm{H}), 7.38(\mathrm{~d}, J=9 \mathrm{~Hz}, 1 \mathrm{H}), 7.09(\mathrm{~s}, 1 \mathrm{H}), 5.78-5.67(\mathrm{~m}, 1 \mathrm{H}), 5.24-4.99(\mathrm{~m}, 1 \mathrm{H}), 5.09(\mathrm{~d}, J=18 \mathrm{~Hz}$, $1 \mathrm{H}), 5.06(\mathrm{~d}, J=11 \mathrm{~Hz}, 1 \mathrm{H}), 4.00-3.92(\mathrm{~m}, 1 \mathrm{H}), 3.92(\mathrm{~s}, 3 \mathrm{H}), 3.79-2.08(\mathrm{~m}, 7 \mathrm{H})$. IR (NaCl, neat) 3849, 3740, 3619, 2927, 2351, 1702, 1516, 1467, 1235, $669 \mathrm{~cm}^{-1}$. HRMS (FAB+) Calcd for $\mathrm{C}_{20} \mathrm{H}_{21} \mathrm{~N}_{2} \mathrm{O}_{2}: 321.1603$. Found 321.1602 $(\mathrm{M}+\mathrm{H})$.<smiles>CCOCC=CCN1CCCC(=O)[C@H]1C(OCCOC(=O)OCC)c1ccnc2ccc(OC)cc12</smiles><smiles>CCOC(=O)OCC=CCN1CCC(C(C)=O)C(=O)[C@H]1C(OCC)c1ccnc2ccc(S(C)(=O)=O)cc12</smiles> 
Carbomethoxy derivative (30): To a solution of $20(54 \mathrm{mg}, 94 \mu \mathrm{mol})$ in THF $(1 \mathrm{~mL})$ at $-78{ }^{\circ} \mathrm{C}$ was added NaHMDS (190 $\mu \mathrm{L}, 0.19 \mathrm{mmol}, 1.0 \mathrm{M}$ solution in THF) and the reaction was stirred for 10 minutes. After which methyl cyanoformate $\left(10 \mu \mathrm{L}, 0.12 \mathrm{mmol}\right.$ ) was added, and after an additional 15 minutes of stirring $\mathrm{NH}_{4} \mathrm{Cl}$ (soln) was added. The reaction mixture was allowed to warm to $\mathrm{rt}$ and diluted with EtOAc. The aqueous layer was extracted with EtOAc and the combined organic layers were washed with brine, dried over $\mathrm{Na}_{2} \mathrm{SO}_{4}$, filtered, and concentrated. The crude product was purified by chromatography on silica gel eluted with 2:1 hexanes/EtOAc to provide $50.0 \mathrm{mg}(81 \mu \mathrm{mol}, 86 \%)$ of $\mathbf{S - 3 0} .{ }^{1} \mathrm{H}$ NMR $\left(\mathrm{CDCl}_{3}, 400 \mathrm{MHz}\right) \delta 12.30(\mathrm{~s}, 1 \mathrm{H}), 8.73(\mathrm{~d}, J=5 \mathrm{~Hz}, 1 \mathrm{H}), 8.07-$ $8.01(\mathrm{~m}, 2 \mathrm{H}), 7.98(\mathrm{~d}, J=9 \mathrm{~Hz}, 1 \mathrm{H}), 7.67-7.42(\mathrm{~m}, 4 \mathrm{H}), 7.31(\mathrm{dd}, J=9 \mathrm{~Hz}, 3 \mathrm{~Hz}, 1 \mathrm{H}), 7.29-7.14(\mathrm{~m}, 1 \mathrm{H}), 5.81-6.58$ $(\mathrm{m}, 3 \mathrm{H}), 5.01-4.75(\mathrm{~m}, 1 \mathrm{H}), 4.72(\mathrm{~d}, J=3 \mathrm{~Hz}, 1 \mathrm{H}), 3.91(\mathrm{~s}, 3 \mathrm{H}), 3.71(\mathrm{~s}, 3 \mathrm{H}), 3.67(\mathrm{~d}, J=3 \mathrm{~Hz}, 1 \mathrm{H}), 3.44-3.18(\mathrm{~m}$, $2 \mathrm{H}), 2.48-2.07(\mathrm{~m}, 3 \mathrm{H}), 1.83-1.48(\mathrm{~m}, 1 \mathrm{H}), 0.86(\mathrm{t}, J=8 \mathrm{~Hz}, 9 \mathrm{H}), 0.54(\mathrm{q}, J=8 \mathrm{~Hz}, 6 \mathrm{H}) .{ }^{13} \mathrm{C}$ NMR $\left(\mathrm{CDCl}_{3}, 100\right.$ MHz) $\delta 220.3,172.3,166.5,157.5,147.3,144.4,138.1,133.2,133.0,131.5,129.8,129.3,128.6,128.4,127.3$, 127.0, 125.5, 121.7, 121.4, 99.0, 66.7, 65.2, 57.1, 55.7, 51.9, 45.2, 18.9, 6.9, 4.9. IR (NaCl, neat) 2954, 2911, 2876, $1720,1659,1621,1508,1442,1271,1238,1225,1109,1070,1027,854,712 \mathrm{~cm}^{-1} .[\alpha]^{25}+63.8\left(c 0.235, \mathrm{CHCl}_{3}\right)$. HRMS (FAB+) Calcd for $\mathrm{C}_{35} \mathrm{H}_{45} \mathrm{~N}_{2} \mathrm{O}_{7} \mathrm{Si}: 633.2996$. Found 633.2984 (M+H).

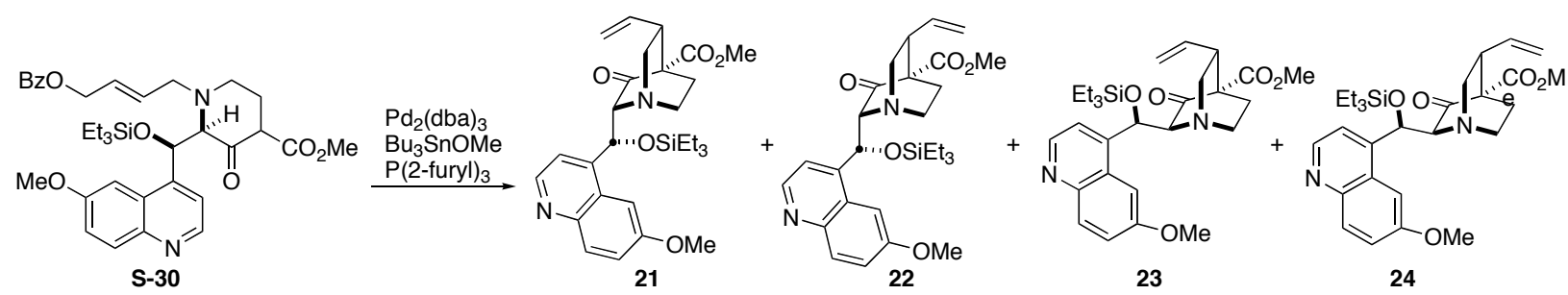

Carbomethoxy cyclization products (21, 22, 23, 24): To a solution of $\mathbf{S - 3 0}(19 \mathrm{mg}, 30 \mu \mathrm{mol})$ in toluene $(5 \mathrm{~mL})$ at room temperature was added tributyltin methoxide $(11 \mu \mathrm{L}, 36 \mu \mathrm{mol})$, and then a solution of $\operatorname{Pd}_{2}\left(\mathrm{dba}_{3}(4 \mathrm{mg}, 4.5\right.$ $\mu \mathrm{mol}), \mathrm{P}(2 \text {-furyl })_{3}(9 \mathrm{mg}, 39 \mu \mathrm{mol})$ in toluene $(5 \mathrm{~mL})$ via cannula. The Schlenck tube was sealed and the reaction was heated to $80-85{ }^{\circ} \mathrm{C}$ for 2.5 hours, and then cooled to $0{ }^{\circ} \mathrm{C}$. To the reaction mixture was added $\mathrm{pH} 7$ buffer $(3$ $\mathrm{mL})$ and the aqueous layer was quickly extracted with EtOAc $(3 \times 5 \mathrm{~mL})$, and the combined organic layers were washed with $\mathrm{NaHCO}_{3}$, filtered through Florosil resin, and partially concentrated (to $\sim 1.5 \mathrm{~mL}$ ). Purification using neutral silica gel chromatography for compounds $\mathbf{2 1}$ and $\mathbf{2 2}$ afforded $0.8 \mathrm{mg}(5 \%)$ of $\mathbf{2 1}$, and $1.7 \mathrm{mg}(11 \%)$ of $\mathbf{2 2}$. Purification of fractions containing epi-C8 compounds $\mathbf{2 1}$ and $\mathbf{2 2}$ using both neutral silica gel chromatography and preparative TLC (9:1 EtOAc/MeOH) afforded $1.4 \mathrm{mg}(9 \%)$ of $\mathbf{2 3}$, and $6.3 \mathrm{mg}(42 \%)$ of $\mathbf{2 4}$. The combined yield of all four diastereomers is $10.2 \mathrm{mg}(67 \%, 20 \mu \mathrm{mol})$ and the diastereoselectivity at the C3-vinyl based on isolated yields is $4: 1$.

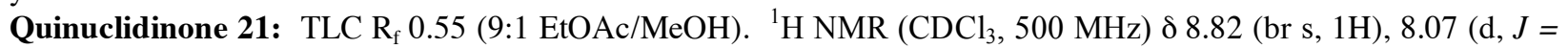
$9 \mathrm{~Hz}, 1 \mathrm{H}), 7.77(\mathrm{~s}, 1 \mathrm{H}), 7.38(\mathrm{dd}, J=9 \mathrm{~Hz}, 3 \mathrm{~Hz}, 1 \mathrm{H}), 7.05(\mathrm{~s}, 1 \mathrm{H}), 5.81(\mathrm{~s}, 1 \mathrm{H}), 5.54(\mathrm{td}, J=17 \mathrm{~Hz}, 9 \mathrm{~Hz}, 1 \mathrm{H})$, 4.96-4.89 (m, 2H), $3.94(\mathrm{~s}, 3 \mathrm{H}), 3.72(\mathrm{~s}, 3 \mathrm{H}), 3.51(\mathrm{br} \mathrm{dd}, J=11 \mathrm{~Hz}, 11 \mathrm{~Hz}, 1 \mathrm{H}), 3.39(\mathrm{dd}, J=13 \mathrm{~Hz}, 11 \mathrm{~Hz}, 1 \mathrm{H})$, $3.35(\mathrm{~s}, 1 \mathrm{H}), 3.04(\mathrm{td}, J=9 \mathrm{~Hz}, 5 \mathrm{~Hz}, 1 \mathrm{H}), 2.94(\mathrm{~m}, 1 \mathrm{H}), 2.66(\mathrm{~d}, J=13 \mathrm{~Hz}, 1 \mathrm{H}), 2.51(\mathrm{td}, J=13 \mathrm{~Hz}, 5 \mathrm{~Hz}, 1 \mathrm{H})$, $2.12(\mathrm{~m}, 1 \mathrm{H}), 0.90(\mathrm{t}, J=8 \mathrm{~Hz}, 9 \mathrm{H}), 0.64-0.55(\mathrm{~m}, 6 \mathrm{H}) .{ }^{13} \mathrm{C} \mathrm{NMR}\left(\mathrm{CDCl}_{3}, 100 \mathrm{MHz}\right.$ and from HSQC, $\mathrm{CDCl}_{3}, 125$ MHz) $\delta 173.4,170.4,158.5,147.9,144.3,139.2,132.4,121.5,119.9,115.7,100.3,74.8,72.6,57.2,56.8,55.9$, 52.2, 45.6, 43.3, 39.6, 29.9, 28.6, 7.0, 5.2. IR (NaCl, neat) 3079, 2953, 2876, 1734, 1621, 1507, 1472, 1434, 1362 , $1238,1114,912,858,794 \mathrm{~cm}^{-1}$. $[\alpha]^{25}{ }_{\mathrm{D}}-73\left(c 0.08, \mathrm{CH}_{2} \mathrm{Cl}_{2}\right)$. HRMS (FAB+) Calcd for $\mathrm{C}_{28} \mathrm{H}_{39} \mathrm{~N}_{2} \mathrm{O}_{5} \mathrm{Si}: 511.2628$. Found $511.2631(\mathrm{M}+\mathrm{H})$.

Quinuclidinone 22: TLC $\mathrm{R}_{\mathrm{f}} 0.65(9: 1 \mathrm{EtOAc} / \mathrm{MeOH}) .{ }^{1} \mathrm{H}$ NMR $\left(\mathrm{CDCl}_{3}, 500 \mathrm{MHz}\right) \delta 8.81(\mathrm{~d}, J=4 \mathrm{~Hz}, 1 \mathrm{H}), 8.07$ $(\mathrm{d}, J=9 \mathrm{~Hz}, 1 \mathrm{H}), 7.79(\mathrm{~d}, J=4 \mathrm{~Hz}, 1 \mathrm{H}), 7.38(\mathrm{dd}, J=9 \mathrm{~Hz}, 2 \mathrm{~Hz}, 1 \mathrm{H}), 7.05$ (br s, 1H), 5.85-5.75 (m, 2H), 5.18$5.10(\mathrm{~m}, 2 \mathrm{H}), 3.94(\mathrm{~s}, 3 \mathrm{H}), 3.70(\mathrm{~s}, 3 \mathrm{H}), 3.53(\mathrm{~s}, 1 \mathrm{H}), 3.52-3.43(\mathrm{~m}, 2 \mathrm{H}), 3.25(\mathrm{dd}, J=12 \mathrm{~Hz}, 12 \mathrm{~Hz}, 1 \mathrm{H}), 3.12-3.05$ $(\mathrm{m}, 1 \mathrm{H}), 2.97-2.83(\mathrm{~m}, 2 \mathrm{H}), 2.34-2.27(\mathrm{~m}, 1 \mathrm{H}), 0.90(\mathrm{t}, J=8 \mathrm{~Hz}, 9 \mathrm{H}), 0.63-0.55(\mathrm{~m}, 6 \mathrm{H}) .{ }^{13} \mathrm{C} \mathrm{NMR}\left(\mathrm{CDCl}_{3}, 100\right.$ $\mathrm{MHz}$ and from $\left.\mathrm{HSQC}, \mathrm{CDCl}_{3}, 125 \mathrm{MHz}\right) \delta 170.1,158.3,147.9,135.1,132.5,121.5,120.0,119.0,100.3,88.8,74.8$, 73.5 (2C), 72.6, 57.8, 56.2, 55.9, 52.3, 44.0, 40.8, 22.9, 22.4, 7.0, 5.1. IR ( $\mathrm{NaCl}$, neat) 2953, 2880, 1738, 1621, $1508,1463,1436,1243,1114,1066,1010,745 \mathrm{~cm}^{-1} .[\alpha]^{25}-81 \quad\left(c 0.17, \mathrm{CH}_{2} \mathrm{Cl}_{2}\right)$. HRMS (FAB+) Calcd for $\mathrm{C}_{28} \mathrm{H}_{39} \mathrm{~N}_{2} \mathrm{O}_{5} \mathrm{Si}: 511.2628$. Found 511.2646 (M+H). 
Quinuclidinone 23: $\mathrm{TLC} \mathrm{R}_{\mathrm{f}} 0.60(9: 1 \mathrm{EtOAc} / \mathrm{MeOH}) .{ }^{1} \mathrm{H} \mathrm{NMR}\left(\mathrm{CDCl}_{3}, 500 \mathrm{MHz}\right) \delta 8.82(\mathrm{~s}, 1 \mathrm{H}), 8.08(\mathrm{~d}, J=9$ $\mathrm{Hz}, 1 \mathrm{H}), 7.65$ (s, 1H), 7.39 (br d, $J=9 \mathrm{~Hz}, 1 \mathrm{H}), 7.08$ (s, 1H), 6.34 (s, 1H), $5.94-5.83$ (m, 1H), 5.07-4.99 (m, 1H), $4.22-4.14(\mathrm{~m}, 1 \mathrm{H}), 3.95(\mathrm{~s}, 3 \mathrm{H}), 3.76(\mathrm{~s}, 3 \mathrm{H}), 3.76(\mathrm{~s}, 3 \mathrm{H}), 3.82-3.71(\mathrm{~m}, 1 \mathrm{H}), 3.83-3.29(\mathrm{~m}, 1 \mathrm{H}), 2.67(\mathrm{dd}, J=11$ $\mathrm{Hz}, 11 \mathrm{~Hz}, 1 \mathrm{H}), 2.29-2.21(\mathrm{~m}, 1 \mathrm{H}), 2.16-2.08(\mathrm{~m}, 1 \mathrm{H}), 0.88(\mathrm{t}, J=8 \mathrm{~Hz}, 9 \mathrm{H}), 0.53(\mathrm{q}, J=8 \mathrm{~Hz}, 6 \mathrm{H})$. IR $(\mathrm{NaCl}$, neat) $2951,2918,2876,1733,1699,1621,1507,1471,1456,1126,1055,1008,920,901,740,668 \mathrm{~cm}^{-1}$. [ $[\alpha]^{25}{ }^{-93}$ (c $0.03, \mathrm{CHCl}_{3}$ ). HRMS (FAB+) Calcd for $\mathrm{C}_{28} \mathrm{H}_{39} \mathrm{~N}_{2} \mathrm{O}_{5} \mathrm{Si}: 511.2628$. Found 511.2629 (M+H).

Quinuclidinone 24: pTLC $\mathrm{R}_{\mathrm{f}} 0.45(9: 1 \mathrm{EtOAc} / \mathrm{MeOH}) .{ }^{1} \mathrm{H} \mathrm{NMR}\left(\mathrm{CDCl}_{3}, 500 \mathrm{MHz}\right) \delta 8.84(\mathrm{~d}, J=4 \mathrm{~Hz}, 1 \mathrm{H})$, $8.09(\mathrm{~d}, J=9 \mathrm{~Hz}, 1 \mathrm{H}), 7.66(\mathrm{~s}, 1 \mathrm{H}), 7.40(\mathrm{dd}, J=9 \mathrm{~Hz}, 2 \mathrm{~Hz}, 1 \mathrm{H}), 7.13$ (br s, $1 \mathrm{H}), 6.19(\mathrm{~s}, 1 \mathrm{H}), 5.83$ (ddd, $J=17$ $\mathrm{Hz}, 10 \mathrm{~Hz}, 9 \mathrm{~Hz}, 1 \mathrm{H}), 5.20$ (d, $J=17 \mathrm{~Hz}, 1 \mathrm{H}), 5.19$ (d, $J=10 \mathrm{~Hz}, 1 \mathrm{H}), 4.22-4.10(\mathrm{~m}, 1 \mathrm{H}), 3.95$ (s, $3 \mathrm{H}), 3.79$ (s, $3 \mathrm{H}), 3.45$ (ddd, $J=8 \mathrm{~Hz}, 8 \mathrm{~Hz}, 8 \mathrm{~Hz}, 1 \mathrm{H}), 3.38$ (d, $J=2 \mathrm{~Hz}, 1 \mathrm{H}), 2.89$ (ddd, $J=14 \mathrm{~Hz}, 11 \mathrm{~Hz}, 5 \mathrm{~Hz}, 1 \mathrm{H}), 2.63-$ $2.58(\mathrm{~m}, 1 \mathrm{H}), 2.57(\mathrm{td}, J=14 \mathrm{~Hz}, 6 \mathrm{~Hz}, 1 \mathrm{H}), 2.31$ (ddd, $J=15 \mathrm{~Hz}, 11 \mathrm{~Hz}, 5 \mathrm{~Hz}, 1 \mathrm{H}), 2.19-2.10(\mathrm{~m}, 1 \mathrm{H}), 2.15$ (dddd, $J=14 \mathrm{~Hz}, 11 \mathrm{~Hz}, 5 \mathrm{~Hz}, 2 \mathrm{~Hz}, 1 \mathrm{H}), 0.90$ (t, $J=8 \mathrm{~Hz}, 9 \mathrm{H}), 0.57$ (q, $J=8 \mathrm{~Hz}, 6 \mathrm{H})$. ${ }^{13} \mathrm{C}$ NMR (obtained from HSQC and $\mathrm{HMBC}$ analysis, $\left.\mathrm{CDCl}_{3}, 125 \mathrm{MHz}\right) \delta 173.4,172.4,160.8,147.7,134.9,132.4,128.0,122.2,121.2$, $119.7,115.4,100.1,88.5,80.0,70.0,59.4,57.9,55.7,53.5,52.2,50.8,22.3,8.9,6.9$. IR (NaCl, neat) 3736,3675 , $3624,2953,2880,1739,1698,1649,1621,1559,1541,1460,1430,1258,1127,1060,1010,751,672 \mathrm{~cm}^{-1} .[\alpha]^{25} \mathrm{D}$ -64 (c 0.17, $\mathrm{CH}_{2} \mathrm{Cl}_{2}$ ). HRMS (FAB+) Calcd for $\mathrm{C}_{28} \mathrm{H}_{39} \mathrm{~N}_{2} \mathrm{O}_{5} \mathrm{Si}$ : 511.2628. Found 511.2641 (M+H). 


\section{X-Ray Crystallography Data for Compound (+/-)-S-43}

Table 1. Crystal data and structure refinement for rw $109 \mathrm{~m}$.

Identification code

Empirical formula

Formula weight

Temperature

Wavelength

Crystal system

Space group

Unit cell dimensions

Volume

Z

Density (calculated)

Absorption coefficient

$\mathrm{F}(000)$

Crystal size

Theta range for data collection

Index ranges

Reflections collected

Independent reflections

Completeness to theta $=28.30^{\circ}$

Absorption correction

Max. and min. transmission

Refinement method

Data / restraints / parameters

Goodness-of-fit on $\mathrm{F}^{2}$

Final $\mathrm{R}$ indices [I $>2 \operatorname{sigma}(\mathrm{I})]$

$\mathrm{R}$ indices (all data)

Largest diff. peak and hole rw109

C37 H32 N2 O7

616.65

373(2) K

$0.71073 \AA$

Monoclinic

$\mathrm{P} 2(1) / \mathrm{c}$

$\mathrm{a}=12.2460(3) \AA$

$\alpha=90^{\circ}$.

$\mathrm{b}=23.6665(6) \AA$

$\beta=113.3980(10)^{\circ}$.

$\mathrm{c}=11.8579(3) \AA$

$\gamma=90^{\circ}$.
3154.05(14) $\AA^{3}$

4

$1.299 \mathrm{Mg} / \mathrm{m}^{3}$

$0.090 \mathrm{~mm}^{-1}$

1296

$0.32 \times 0.23 \times 0.09 \mathrm{~mm}^{3}$

1.81 to $28.30^{\circ}$.

$-16<=\mathrm{h}<=16,-31<=\mathrm{k}<=31,-15<=1<=15$

33634

$7836[\mathrm{R}($ int $)=0.0541]$

$99.8 \%$

multi-scan

0.9920 and 0.9718

Full-matrix least-squares on $\mathrm{F}^{2}$

$7836 / 0 / 415$

1.007

$\mathrm{R} 1=0.0504, \mathrm{wR} 2=0.1184$

$\mathrm{R} 1=0.0858, \mathrm{wR} 2=0.1365$

0.761 and -0.263 e. $\AA^{-3}$ 
Table 2. Atomic coordinates ( $\left.\times 10^{4}\right)$ and equivalent isotropic displacement parameters $\left(\AA^{2} \times 10^{3}\right)$ for rw $109 \mathrm{~m}$. $\mathrm{U}(\mathrm{eq})$ is defined as one third of the trace of the orthogonalized $\mathrm{U}^{\mathrm{ij}}$ tensor.

\begin{tabular}{|c|c|c|c|c|}
\hline & $\mathrm{x}$ & $\mathrm{y}$ & z & $\mathrm{U}(\mathrm{eq})$ \\
\hline $\mathrm{O}(1)$ & $7230(1)$ & 2861(1) & 1333(1) & $18(1)$ \\
\hline $\mathrm{O}(2)$ & $6814(1)$ & $2172(1)$ & $2338(1)$ & $21(1)$ \\
\hline $\mathrm{O}(3)$ & 3992(1) & 2833(1) & $-2708(1)$ & $21(1)$ \\
\hline $\mathrm{O}(4)$ & $3707(1)$ & 1997(1) & $-1930(1)$ & $21(1)$ \\
\hline $\mathrm{O}(5)$ & $7292(1)$ & 1761(1) & $-193(1)$ & $18(1)$ \\
\hline $\mathrm{O}(6)$ & $7060(1)$ & 1074(1) & $-1577(1)$ & $27(1)$ \\
\hline $\mathrm{O}(7)$ & 2019(1) & $733(1)$ & $-670(1)$ & $27(1)$ \\
\hline $\mathrm{N}(1)$ & $5299(1)$ & $2557(1)$ & $-853(1)$ & $16(1)$ \\
\hline $\mathrm{N}(2)$ & $6659(1)$ & $160(1)$ & $2282(1)$ & $23(1)$ \\
\hline $\mathrm{C}(1)$ & $5684(2)$ & 2153(1) & $176(2)$ & $16(1)$ \\
\hline$C(2)$ & $6618(2)$ & $2396(1)$ & $1365(2)$ & $16(1)$ \\
\hline$C(3)$ & $7110(2)$ & $3076(1)$ & $130(2)$ & $16(1)$ \\
\hline$C(4)$ & $5799(2)$ & $3132(1)$ & $-711(2)$ & $16(1)$ \\
\hline$C(5)$ & $5068(2)$ & $3556(1)$ & $-341(2)$ & $16(1)$ \\
\hline$C(6)$ & $4772(2)$ & $4068(1)$ & $-967(2)$ & $21(1)$ \\
\hline$C(7)$ & 4091(2) & $4466(1)$ & $-675(2)$ & $25(1)$ \\
\hline$C(8)$ & $3698(2)$ & $4360(1)$ & $250(2)$ & $24(1)$ \\
\hline$C(9)$ & $3979(2)$ & $3851(1)$ & $873(2)$ & $24(1)$ \\
\hline $\mathrm{C}(10)$ & $4652(2)$ & $3450(1)$ & $579(2)$ & $21(1)$ \\
\hline $\mathrm{C}(11)$ & $7847(2)$ & $3606(1)$ & $367(2)$ & $18(1)$ \\
\hline$C(12)$ & $8734(2)$ & $3639(1)$ & $-90(2)$ & $24(1)$ \\
\hline$C(13)$ & $9466(2)$ & $4108(1)$ & $149(2)$ & $30(1)$ \\
\hline$C(14)$ & $9317(2)$ & $4550(1)$ & $838(2)$ & $30(1)$ \\
\hline$C(15)$ & $8440(2)$ & $4520(1)$ & $1296(2)$ & $27(1)$ \\
\hline$C(16)$ & $7708(2)$ & $4051(1)$ & $1065(2)$ & $22(1)$ \\
\hline$C(17)$ & $4278(2)$ & $2427(1)$ & $-1836(2)$ & $17(1)$ \\
\hline$C(18)$ & $2824(2)$ & $2768(1)$ & $-3705(2)$ & $23(1)$ \\
\hline$C(19)$ & $1848(2)$ & $2864(1)$ & $-3261(2)$ & $22(1)$ \\
\hline $\mathrm{C}(20)$ & $1765(2)$ & $3379(1)$ & $-2742(2)$ & $40(1)$ \\
\hline$C(21)$ & $869(2)$ & $3462(1)$ & $-2311(2)$ & $54(1)$ \\
\hline $\mathrm{C}(22)$ & $70(2)$ & $3035(1)$ & $-2402(2)$ & $47(1)$ \\
\hline
\end{tabular}




\begin{tabular}{lrrrr}
$\mathrm{C}(23)$ & $146(2)$ & $2524(1)$ & $-2926(2)$ & $35(1)$ \\
$\mathrm{C}(24)$ & $1033(2)$ & $2442(1)$ & $-3352(2)$ & $26(1)$ \\
$\mathrm{C}(25)$ & $6165(1)$ & $1602(1)$ & $-159(2)$ & $16(1)$ \\
$\mathrm{C}(26)$ & $7650(2)$ & $1449(1)$ & $-952(2)$ & $19(1)$ \\
$\mathrm{C}(27)$ & $8848(2)$ & $1637(1)$ & $-859(2)$ & $25(1)$ \\
$\mathrm{C}(28)$ & $6322(2)$ & $1111(1)$ & $717(2)$ & $18(1)$ \\
$\mathrm{C}(29)$ & $7431(2)$ & $923(1)$ & $1470(2)$ & $21(1)$ \\
$\mathrm{C}(30)$ & $7550(2)$ & $448(1)$ & $2225(2)$ & $25(1)$ \\
$\mathrm{C}(31)$ & $5541(2)$ & $339(1)$ & $1539(2)$ & $21(1)$ \\
$\mathrm{C}(32)$ & $4563(2)$ & $29(1)$ & $1573(2)$ & $23(1)$ \\
$\mathrm{C}(33)$ & $3423(2)$ & $179(1)$ & $850(2)$ & $24(1)$ \\
$\mathrm{C}(34)$ & $3199(2)$ & $640(1)$ & $34(2)$ & $22(1)$ \\
$\mathrm{C}(35)$ & $4118(2)$ & $950(1)$ & $-37(2)$ & $19(1)$ \\
$\mathrm{C}(36)$ & $5315(2)$ & $811(1)$ & $730(2)$ & $18(1)$ \\
$\mathrm{C}(37)$ & $1743(2)$ & $1133(1)$ & $-1642(2)$ & $28(1)$ \\
\hline
\end{tabular}


Table 3. Bond lengths $[\AA]$ and angles $\left[^{\circ}\right]$ for rw $109 \mathrm{~m}$.

\begin{tabular}{|c|c|c|c|}
\hline $\mathrm{O}(1)-\mathrm{C}(2)$ & $1.340(2)$ & $\mathrm{C}(9)-\mathrm{C}(10)$ & $1.390(3)$ \\
\hline $\mathrm{O}(1)-\mathrm{C}(3)$ & $1.4667(19)$ & $\mathrm{C}(11)-\mathrm{C}(16)$ & $1.390(3)$ \\
\hline $\mathrm{O}(2)-\mathrm{C}(2)$ & $1.204(2)$ & $\mathrm{C}(11)-\mathrm{C}(12)$ & $1.395(2)$ \\
\hline $\mathrm{O}(3)-\mathrm{C}(17)$ & $1.353(2)$ & $\mathrm{C}(12)-\mathrm{C}(13)$ & $1.383(3)$ \\
\hline $\mathrm{O}(3)-\mathrm{C}(18)$ & $1.458(2)$ & $\mathrm{C}(13)-\mathrm{C}(14)$ & $1.383(3)$ \\
\hline $\mathrm{O}(4)-\mathrm{C}(17)$ & $1.214(2)$ & $\mathrm{C}(14)-\mathrm{C}(15)$ & $1.384(3)$ \\
\hline $\mathrm{O}(5)-\mathrm{C}(26)$ & $1.365(2)$ & $C(15)-C(16)$ & $1.384(3)$ \\
\hline $\mathrm{O}(5)-\mathrm{C}(25)$ & $1.446(2)$ & $\mathrm{C}(18)-\mathrm{C}(19)$ & $1.503(3)$ \\
\hline $\mathrm{O}(6)-\mathrm{C}(26)$ & $1.197(2)$ & $C(19)-C(24)$ & $1.386(3)$ \\
\hline $\mathrm{O}(7)-\mathrm{C}(34)$ & $1.369(2)$ & $C(19)-C(20)$ & $1.386(3)$ \\
\hline $\mathrm{O}(7)-\mathrm{C}(37)$ & $1.425(2)$ & $C(20)-C(21)$ & $1.396(3)$ \\
\hline $\mathrm{N}(1)-\mathrm{C}(17)$ & $1.364(2)$ & $C(21)-C(22)$ & $1.380(4)$ \\
\hline $\mathrm{N}(1)-\mathrm{C}(1)$ & $1.471(2)$ & $C(22)-C(23)$ & $1.380(4)$ \\
\hline $\mathrm{N}(1)-\mathrm{C}(4)$ & $1.475(2)$ & $C(23)-C(24)$ & $1.382(3)$ \\
\hline $\mathrm{N}(2)-\mathrm{C}(30)$ & $1.311(2)$ & $C(25)-C(28)$ & $1.519(2)$ \\
\hline $\mathrm{N}(2)-\mathrm{C}(31)$ & $1.369(2)$ & $C(26)-C(27)$ & $1.495(2)$ \\
\hline$C(1)-C(2)$ & $1.532(2)$ & $C(28)-C(29)$ & $1.370(2)$ \\
\hline$C(1)-C(25)$ & $1.547(2)$ & $C(28)-C(36)$ & $1.428(2)$ \\
\hline$C(3)-C(11)$ & $1.506(2)$ & $C(29)-C(30)$ & $1.409(3)$ \\
\hline $\mathrm{C}(3)-\mathrm{C}(4)$ & $1.523(2)$ & $\mathrm{C}(31)-\mathrm{C}(32)$ & $1.420(3)$ \\
\hline $\mathrm{C}(4)-\mathrm{C}(5)$ & $1.522(2)$ & $C(31)-C(36)$ & $1.426(3)$ \\
\hline$C(5)-C(6)$ & $1.390(3)$ & $C(32)-C(33)$ & $1.364(3)$ \\
\hline$C(5)-C(10)$ & $1.398(2)$ & $\mathrm{C}(33)-\mathrm{C}(34)$ & $1.410(3)$ \\
\hline$C(6)-C(7)$ & $1.390(3)$ & $C(34)-C(35)$ & $1.374(3)$ \\
\hline $\mathrm{C}(7)-\mathrm{C}(8)$ & $1.385(3)$ & $C(35)-C(36)$ & $1.423(2)$ \\
\hline $\mathrm{C}(8)-\mathrm{C}(9)$ & $1.382(3)$ & & \\
\hline $\mathrm{C}(2)-\mathrm{O}(1)-\mathrm{C}(3)$ & $118.11(13)$ & $\mathrm{C}(30)-\mathrm{N}(2)-\mathrm{C}(31)$ & $116.46(16)$ \\
\hline $\mathrm{C}(17)-\mathrm{O}(3)-\mathrm{C}(18)$ & $114.66(14)$ & $\mathrm{N}(1)-\mathrm{C}(1)-\mathrm{C}(2)$ & $113.35(14)$ \\
\hline $\mathrm{C}(26)-\mathrm{O}(5)-\mathrm{C}(25)$ & $115.98(13)$ & $\mathrm{N}(1)-\mathrm{C}(1)-\mathrm{C}(25)$ & $111.28(13)$ \\
\hline $\mathrm{C}(34)-\mathrm{O}(7)-\mathrm{C}(37)$ & $116.95(14)$ & $\mathrm{C}(2)-\mathrm{C}(1)-\mathrm{C}(25)$ & $109.35(14)$ \\
\hline $\mathrm{C}(17)-\mathrm{N}(1)-\mathrm{C}(1)$ & $116.51(14)$ & $\mathrm{O}(2)-\mathrm{C}(2)-\mathrm{O}(1)$ & $119.20(16)$ \\
\hline $\mathrm{C}(17)-\mathrm{N}(1)-\mathrm{C}(4)$ & $120.23(14)$ & $\mathrm{O}(2)-\mathrm{C}(2)-\mathrm{C}(1)$ & $120.55(16)$ \\
\hline $\mathrm{C}(1)-\mathrm{N}(1)-\mathrm{C}(4)$ & $121.49(13)$ & $\mathrm{O}(1)-\mathrm{C}(2)-\mathrm{C}(1)$ & $120.25(14)$ \\
\hline
\end{tabular}




\begin{tabular}{|c|c|c|c|}
\hline $\mathrm{O}(1)-\mathrm{C}(3)-\mathrm{C}(11)$ & $106.65(13)$ & $\mathrm{C}(28)-\mathrm{C}(25)-\mathrm{C}(1)$ & $115.28(14)$ \\
\hline $\mathrm{O}(1)-\mathrm{C}(3)-\mathrm{C}(4)$ & $109.83(13)$ & $\mathrm{O}(6)-\mathrm{C}(26)-\mathrm{O}(5)$ & $122.44(16)$ \\
\hline$C(11)-C(3)-C(4)$ & $116.54(15)$ & $\mathrm{O}(6)-\mathrm{C}(26)-\mathrm{C}(27)$ & $126.87(17)$ \\
\hline $\mathrm{N}(1)-\mathrm{C}(4)-\mathrm{C}(5)$ & $112.10(13)$ & $\mathrm{O}(5)-\mathrm{C}(26)-\mathrm{C}(27)$ & $110.68(16)$ \\
\hline $\mathrm{N}(1)-\mathrm{C}(4)-\mathrm{C}(3)$ & $106.05(14)$ & $C(29)-C(28)-C(36)$ & $117.93(17)$ \\
\hline$C(5)-C(4)-C(3)$ & $117.22(14)$ & $\mathrm{C}(29)-\mathrm{C}(28)-\mathrm{C}(25)$ & $121.20(16)$ \\
\hline$C(6)-C(5)-C(10)$ & $118.35(16)$ & $C(36)-C(28)-C(25)$ & $120.79(15)$ \\
\hline$C(6)-C(5)-C(4)$ & $118.64(15)$ & $\mathrm{C}(28)-\mathrm{C}(29)-\mathrm{C}(30)$ & $119.95(17)$ \\
\hline$C(10)-C(5)-C(4)$ & $123.00(16)$ & $\mathrm{N}(2)-\mathrm{C}(30)-\mathrm{C}(29)$ & $124.74(18)$ \\
\hline$C(5)-C(6)-C(7)$ & $120.86(17)$ & $\mathrm{N}(2)-\mathrm{C}(31)-\mathrm{C}(32)$ & $117.40(17)$ \\
\hline$C(8)-C(7)-C(6)$ & $120.32(18)$ & $\mathrm{N}(2)-\mathrm{C}(31)-\mathrm{C}(36)$ & $123.63(17)$ \\
\hline$C(9)-C(8)-C(7)$ & $119.36(17)$ & $C(32)-C(31)-C(36)$ & $118.96(17)$ \\
\hline$C(8)-C(9)-C(10)$ & $120.54(17)$ & $C(33)-C(32)-C(31)$ & $120.73(18)$ \\
\hline$C(9)-C(10)-C(5)$ & $120.55(17)$ & $C(32)-C(33)-C(34)$ & $120.36(17)$ \\
\hline $\mathrm{C}(16)-\mathrm{C}(11)-\mathrm{C}(12)$ & $119.12(17)$ & $\mathrm{O}(7)-\mathrm{C}(34)-\mathrm{C}(35)$ & $124.66(17)$ \\
\hline$C(16)-C(11)-C(3)$ & $122.47(16)$ & $\mathrm{O}(7)-\mathrm{C}(34)-\mathrm{C}(33)$ & $114.41(16)$ \\
\hline$C(12)-C(11)-C(3)$ & $118.35(17)$ & $\mathrm{C}(35)-\mathrm{C}(34)-\mathrm{C}(33)$ & $120.91(17)$ \\
\hline $\mathrm{C}(13)-\mathrm{C}(12)-\mathrm{C}(11)$ & $120.43(19)$ & $C(34)-C(35)-C(36)$ & 119.93(17) \\
\hline$C(14)-C(13)-C(12)$ & $120.06(19)$ & $C(35)-C(36)-C(31)$ & $119.07(16)$ \\
\hline $\mathrm{C}(13)-\mathrm{C}(14)-\mathrm{C}(15)$ & $119.88(19)$ & $C(35)-C(36)-C(28)$ & $123.60(16)$ \\
\hline $\mathrm{C}(14)-\mathrm{C}(15)-\mathrm{C}(16)$ & $120.34(19)$ & $\mathrm{C}(31)-\mathrm{C}(36)-\mathrm{C}(28)$ & $117.30(16)$ \\
\hline$C(15)-C(16)-C(11)$ & $120.18(18)$ & & \\
\hline $\mathrm{O}(4)-\mathrm{C}(17)-\mathrm{O}(3)$ & $124.16(16)$ & & \\
\hline $\mathrm{O}(4)-\mathrm{C}(17)-\mathrm{N}(1)$ & $124.45(16)$ & & \\
\hline $\mathrm{O}(3)-\mathrm{C}(17)-\mathrm{N}(1)$ & $111.37(15)$ & & \\
\hline $\mathrm{O}(3)-\mathrm{C}(18)-\mathrm{C}(19)$ & $111.14(14)$ & & \\
\hline $\mathrm{C}(24)-\mathrm{C}(19)-\mathrm{C}(20)$ & $119.43(19)$ & & \\
\hline $\mathrm{C}(24)-\mathrm{C}(19)-\mathrm{C}(18)$ & $120.79(18)$ & & \\
\hline $\mathrm{C}(20)-\mathrm{C}(19)-\mathrm{C}(18)$ & $119.78(18)$ & & \\
\hline $\mathrm{C}(19)-\mathrm{C}(20)-\mathrm{C}(21)$ & $119.5(2)$ & & \\
\hline $\mathrm{C}(22)-\mathrm{C}(21)-\mathrm{C}(20)$ & $120.3(2)$ & & \\
\hline $\mathrm{C}(23)-\mathrm{C}(22)-\mathrm{C}(21)$ & $120.3(2)$ & & \\
\hline $\mathrm{C}(22)-\mathrm{C}(23)-\mathrm{C}(24)$ & $119.5(2)$ & & \\
\hline $\mathrm{C}(23)-\mathrm{C}(24)-\mathrm{C}(19)$ & $121.0(2)$ & & \\
\hline $\mathrm{O}(5)-\mathrm{C}(25)-\mathrm{C}(28)$ & $110.72(14)$ & & \\
\hline $\mathrm{O}(5)-\mathrm{C}(25)-\mathrm{C}(1)$ & $104.67(13)$ & & \\
\hline
\end{tabular}


Table 4. Anisotropic displacement parameters $\left(\AA^{2} \times 10^{3}\right)$ for rw109m. The anisotropic displacement factor exponent takes the form: $-2 \pi^{2}\left[h^{2} a^{* 2} U^{11}+\ldots+2 h k a^{*} b^{*} U^{12}\right]$

\begin{tabular}{|c|c|c|c|c|c|c|}
\hline & $\mathrm{U}^{11}$ & $\mathrm{U}^{22}$ & $\mathrm{U}^{33}$ & $U^{23}$ & $\mathrm{U}^{13}$ & $\mathrm{U}^{12}$ \\
\hline $\mathrm{O}(1)$ & $20(1)$ & $18(1)$ & $15(1)$ & 1(1) & $7(1)$ & $-3(1)$ \\
\hline $\mathrm{O}(2)$ & $25(1)$ & $23(1)$ & $17(1)$ & 2(1) & $10(1)$ & $-2(1)$ \\
\hline $\mathrm{O}(3)$ & $16(1)$ & $26(1)$ & $18(1)$ & $5(1)$ & $4(1)$ & $-1(1)$ \\
\hline $\mathrm{O}(4)$ & $19(1)$ & $19(1)$ & $22(1)$ & $-1(1)$ & $5(1)$ & $-3(1)$ \\
\hline $\mathrm{O}(5)$ & $17(1)$ & $18(1)$ & $20(1)$ & $-1(1)$ & $9(1)$ & $-1(1)$ \\
\hline $\mathrm{O}(6)$ & $34(1)$ & $25(1)$ & $29(1)$ & $-8(1)$ & $19(1)$ & $-7(1)$ \\
\hline $\mathrm{O}(7)$ & 21(1) & $28(1)$ & $33(1)$ & 2(1) & 11(1) & $-4(1)$ \\
\hline $\mathrm{N}(1)$ & $17(1)$ & $14(1)$ & $16(1)$ & 2(1) & $6(1)$ & $-1(1)$ \\
\hline $\mathrm{N}(2)$ & $30(1)$ & $20(1)$ & $18(1)$ & 1(1) & $6(1)$ & $1(1)$ \\
\hline$C(1)$ & $16(1)$ & $16(1)$ & $17(1)$ & $2(1)$ & $8(1)$ & $-1(1)$ \\
\hline$C(2)$ & $16(1)$ & $16(1)$ & $18(1)$ & $-1(1)$ & $9(1)$ & $2(1)$ \\
\hline$C(3)$ & 19(1) & $17(1)$ & $15(1)$ & $2(1)$ & $9(1)$ & $0(1)$ \\
\hline$C(4)$ & 19(1) & $14(1)$ & $16(1)$ & 1(1) & $8(1)$ & $-2(1)$ \\
\hline$C(5)$ & $16(1)$ & $17(1)$ & $17(1)$ & $-1(1)$ & $7(1)$ & $-1(1)$ \\
\hline$C(6)$ & $27(1)$ & 19(1) & $20(1)$ & 1(1) & $11(1)$ & $-1(1)$ \\
\hline$C(7)$ & $31(1)$ & $17(1)$ & $27(1)$ & $4(1)$ & $12(1)$ & $4(1)$ \\
\hline$C(8)$ & $25(1)$ & $22(1)$ & $26(1)$ & $-4(1)$ & $11(1)$ & $4(1)$ \\
\hline$C(9)$ & $26(1)$ & $27(1)$ & $22(1)$ & 1(1) & $14(1)$ & $2(1)$ \\
\hline$C(10)$ & $23(1)$ & $20(1)$ & 21(1) & $4(1)$ & $11(1)$ & $2(1)$ \\
\hline $\mathrm{C}(11)$ & $18(1)$ & 19(1) & $16(1)$ & 2(1) & $6(1)$ & $-2(1)$ \\
\hline $\mathrm{C}(12)$ & $24(1)$ & 29(1) & $22(1)$ & $0(1)$ & $10(1)$ & $-5(1)$ \\
\hline$C(13)$ & $25(1)$ & $36(1)$ & $29(1)$ & $7(1)$ & $12(1)$ & $-9(1)$ \\
\hline$C(14)$ & $26(1)$ & $26(1)$ & $29(1)$ & $8(1)$ & $2(1)$ & $-11(1)$ \\
\hline$C(15)$ & $29(1)$ & $20(1)$ & $26(1)$ & $-1(1)$ & $5(1)$ & $-2(1)$ \\
\hline$C(16)$ & $23(1)$ & 21(1) & $22(1)$ & 1(1) & $8(1)$ & $-1(1)$ \\
\hline$C(17)$ & $16(1)$ & $18(1)$ & $19(1)$ & 1(1) & $9(1)$ & $2(1)$ \\
\hline$C(18)$ & $16(1)$ & $31(1)$ & $17(1)$ & $5(1)$ & $2(1)$ & $0(1)$ \\
\hline$C(19)$ & $17(1)$ & $28(1)$ & $18(1)$ & 2(1) & $3(1)$ & $3(1)$ \\
\hline$C(20)$ & $27(1)$ & $41(1)$ & $47(1)$ & $-14(1)$ & $9(1)$ & $0(1)$ \\
\hline
\end{tabular}




\begin{tabular}{lllllll}
$\mathrm{C}(21)$ & $41(1)$ & $65(2)$ & $50(2)$ & $-23(1)$ & $13(1)$ & $14(1)$ \\
$\mathrm{C}(22)$ & $29(1)$ & $82(2)$ & $33(1)$ & $-1(1)$ & $16(1)$ & $11(1)$ \\
$\mathrm{C}(23)$ & $21(1)$ & $51(2)$ & $34(1)$ & $13(1)$ & $11(1)$ & $5(1)$ \\
$\mathrm{C}(24)$ & $20(1)$ & $32(1)$ & $25(1)$ & $5(1)$ & $6(1)$ & $4(1)$ \\
$\mathrm{C}(25)$ & $13(1)$ & $19(1)$ & $16(1)$ & $-1(1)$ & $5(1)$ & $-2(1)$ \\
$\mathrm{C}(26)$ & $24(1)$ & $18(1)$ & $18(1)$ & $2(1)$ & $11(1)$ & $2(1)$ \\
$\mathrm{C}(27)$ & $24(1)$ & $29(1)$ & $26(1)$ & $-1(1)$ & $14(1)$ & $0(1)$ \\
$\mathrm{C}(28)$ & $23(1)$ & $15(1)$ & $16(1)$ & $-2(1)$ & $7(1)$ & $0(1)$ \\
$\mathrm{C}(29)$ & $21(1)$ & $20(1)$ & $20(1)$ & $0(1)$ & $5(1)$ & $-2(1)$ \\
$\mathrm{C}(30)$ & $25(1)$ & $23(1)$ & $20(1)$ & $0(1)$ & $2(1)$ & $2(1)$ \\
$\mathrm{C}(31)$ & $28(1)$ & $17(1)$ & $18(1)$ & $-4(1)$ & $10(1)$ & $-1(1)$ \\
$\mathrm{C}(32)$ & $35(1)$ & $16(1)$ & $22(1)$ & $-1(1)$ & $16(1)$ & $-4(1)$ \\
$\mathrm{C}(33)$ & $31(1)$ & $20(1)$ & $28(1)$ & $-4(1)$ & $18(1)$ & $-7(1)$ \\
$\mathrm{C}(34)$ & $23(1)$ & $20(1)$ & $23(1)$ & $-5(1)$ & $11(1)$ & $-3(1)$ \\
$\mathrm{C}(35)$ & $23(1)$ & $16(1)$ & $19(1)$ & $-1(1)$ & $10(1)$ & $-1(1)$ \\
$\mathrm{C}(36)$ & $24(1)$ & $15(1)$ & $16(1)$ & $-3(1)$ & $10(1)$ & $-2(1)$ \\
$\mathrm{C}(37)$ & $21(1)$ & $35(1)$ & $26(1)$ & $-1(1)$ & $5(1)$ & $-3(1)$ \\
& & & & & & \\
\hline
\end{tabular}


Table 5. Hydrogen coordinates ( $\left.\mathrm{x} 10^{4}\right)$ and isotropic displacement parameters $\left(\AA^{2} \times 10^{3}\right)$ for rw $109 \mathrm{~m}$.

\begin{tabular}{|c|c|c|c|c|}
\hline & $\mathrm{x}$ & $\mathrm{y}$ & $\mathrm{z}$ & $\mathrm{U}(\mathrm{eq})$ \\
\hline $\mathrm{H}(1 \mathrm{~A})$ & 4983 & 2054 & 339 & 19 \\
\hline $\mathrm{H}(3 \mathrm{~A})$ & 7465 & 2797 & -236 & 20 \\
\hline $\mathrm{H}(4 \mathrm{~A})$ & 5762 & 3248 & -1519 & 19 \\
\hline $\mathrm{H}(6 \mathrm{~A})$ & 5032 & 4144 & -1589 & 26 \\
\hline $\mathrm{H}(7 \mathrm{~A})$ & 3900 & 4806 & -1103 & 30 \\
\hline $\mathrm{H}(8 \mathrm{~A})$ & 3248 & 4628 & 450 & 29 \\
\hline $\mathrm{H}(9 \mathrm{~A})$ & 3715 & 3777 & 1493 & 28 \\
\hline $\mathrm{H}(10 \mathrm{~A})$ & 4827 & 3107 & 998 & 25 \\
\hline $\mathrm{H}(12 \mathrm{~A})$ & 8834 & 3344 & -557 & 29 \\
\hline $\mathrm{H}(13 \mathrm{~A})$ & 10058 & 4126 & -154 & 36 \\
\hline $\mathrm{H}(14 \mathrm{~A})$ & 9805 & 4866 & 994 & 36 \\
\hline $\mathrm{H}(15 \mathrm{~A})$ & 8342 & 4817 & 1762 & 33 \\
\hline $\mathrm{H}(16 \mathrm{~A})$ & 7123 & 4033 & 1378 & 26 \\
\hline $\mathrm{H}(18 \mathrm{~A})$ & 2752 & 2391 & -4046 & 27 \\
\hline $\mathrm{H}(18 \mathrm{~B})$ & 2742 & 3037 & -4352 & 27 \\
\hline $\mathrm{H}(20 \mathrm{~A})$ & 2301 & 3667 & -2683 & 48 \\
\hline $\mathrm{H}(21 \mathrm{~A})$ & 810 & 3806 & -1961 & 64 \\
\hline $\mathrm{H}(22 \mathrm{~A})$ & -523 & 3092 & -2109 & 56 \\
\hline $\mathrm{H}(23 \mathrm{~A})$ & -394 & 2237 & -2991 & 42 \\
\hline $\mathrm{H}(24 \mathrm{~A})$ & 1084 & 2098 & -3707 & 32 \\
\hline $\mathrm{H}(25 \mathrm{~A})$ & 5623 & 1485 & -986 & 20 \\
\hline $\mathrm{H}(27 \mathrm{~A})$ & 9088 & 1411 & -1394 & 38 \\
\hline $\mathrm{H}(27 \mathrm{~B})$ & 9416 & 1594 & -27 & 38 \\
\hline $\mathrm{H}(27 \mathrm{C})$ & 8810 & 2027 & -1096 & 38 \\
\hline $\mathrm{H}(29 \mathrm{~A})$ & 8105 & 1110 & 1484 & 25 \\
\hline $\mathrm{H}(30 \mathrm{~A})$ & 8316 & 329 & 2718 & 30 \\
\hline $\mathrm{H}(32 \mathrm{~A})$ & 4701 & -281 & 2094 & 28 \\
\hline $\mathrm{H}(33 \mathrm{~A})$ & 2791 & -23 & 895 & 29 \\
\hline $\mathrm{H}(35 \mathrm{~A})$ & 3958 & 1250 & -586 & 23 \\
\hline $\mathrm{H}(37 \mathrm{~A})$ & 896 & 1163 & -2068 & 43 \\
\hline
\end{tabular}


H(37B)

2084

1011

$-2204$

43

$\mathrm{H}(37 \mathrm{C})$

2066

1495

$-1309$

43 


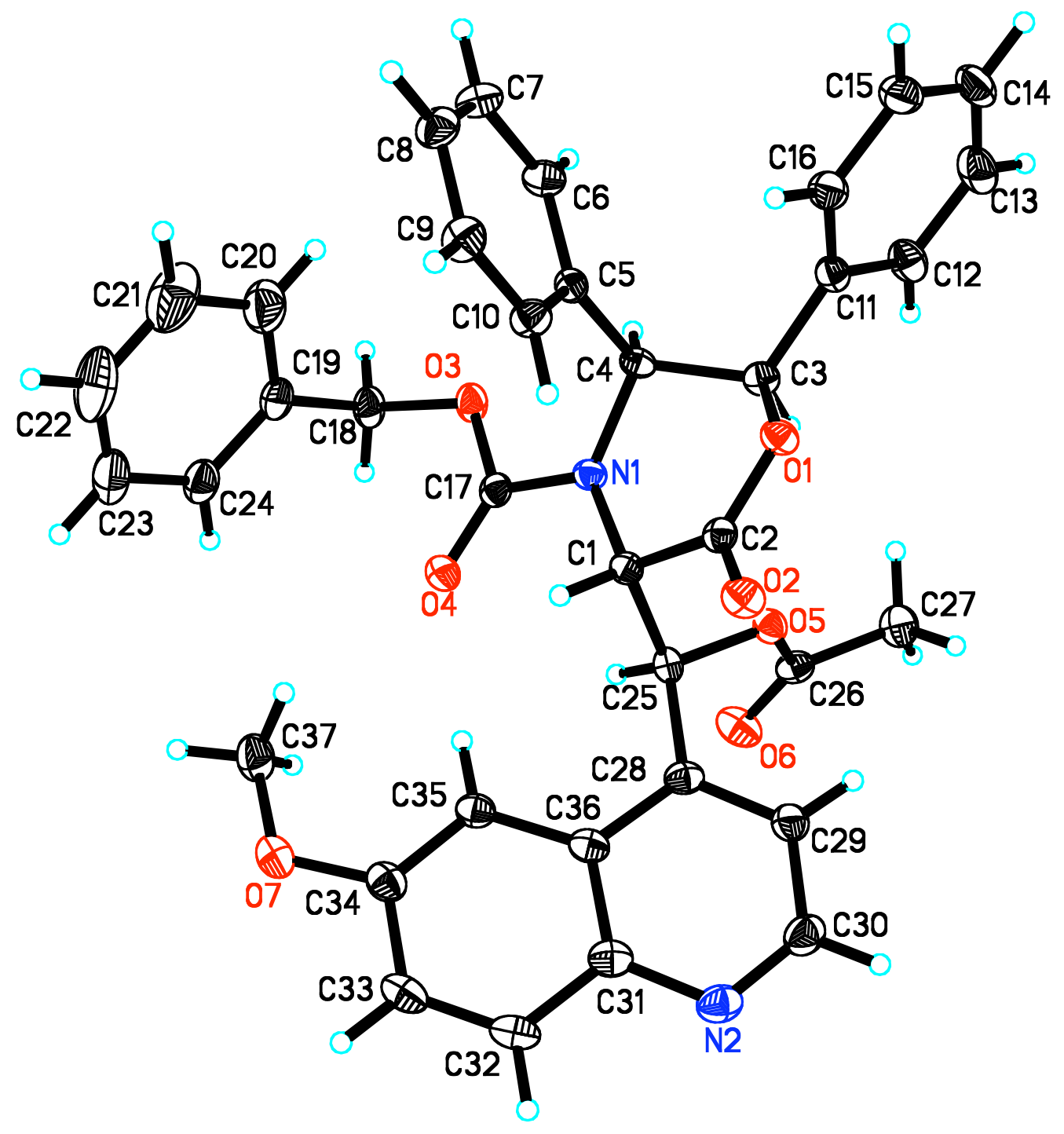




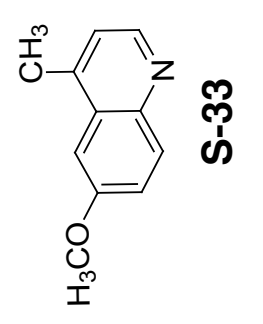

Page S27

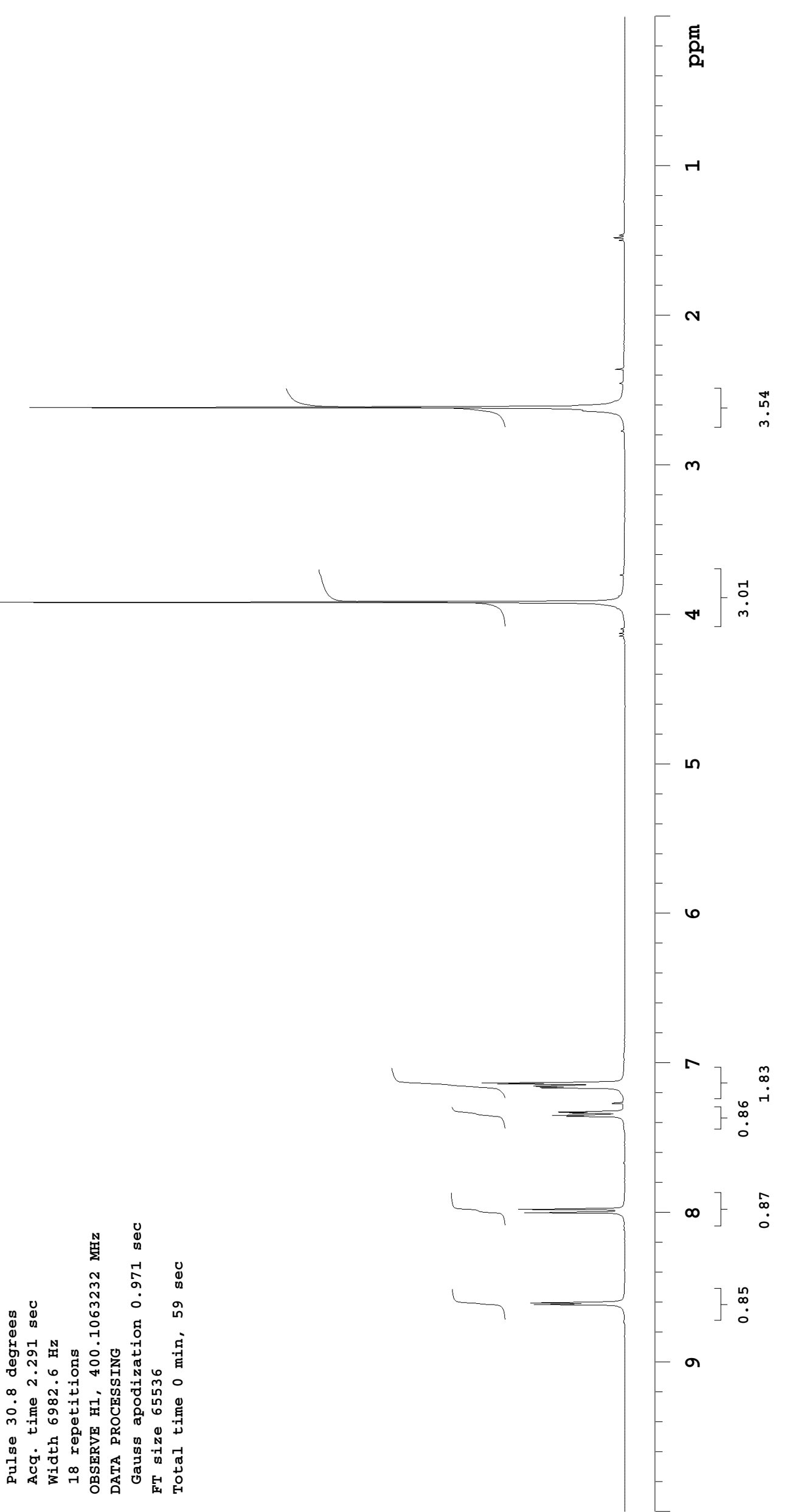




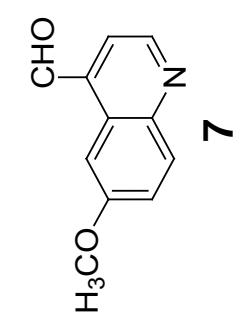

Page S29

్ㅗㅇ

N

$\stackrel{\infty}{\stackrel{\infty}{0}}$

m

$+1 \stackrel{\circ}{\dot{m}}$ 
$\infty$

$9 L S^{\cdot} S \mathrm{~T}$

ع96.8 8

$8 \mathrm{Z} \cdot 09$

Ð0S $\angle 9$

$6 S L \cdot L 9$

$206 \cdot 9$

$0 \varepsilon Z \cdot L L \longrightarrow$

$0 Ð S^{\circ} L L$

ฤ乙: 6

sโz 6

$8 \varepsilon \tau \cdot L 8$

Zโ0.88

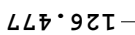

ह乙9・9乙Т

$909 \cdot 1 Z T$

$908^{\circ} L Z$

T98 $\mathrm{LZT}$

$686^{\circ} \mathrm{LZT}$

$\varepsilon \varpi 0 \cdot 8 乙 \tau$

$860^{\circ} 8 \mathrm{ZT}$

ZST・8ZT

$68 \tau \cdot 8 \mathrm{ZT}$

$08 Z \cdot 8 Z \tau$

$9 \tau \varepsilon \cdot 8 Z \tau$

$Z 9 \varpi \cdot 8 Z \tau$

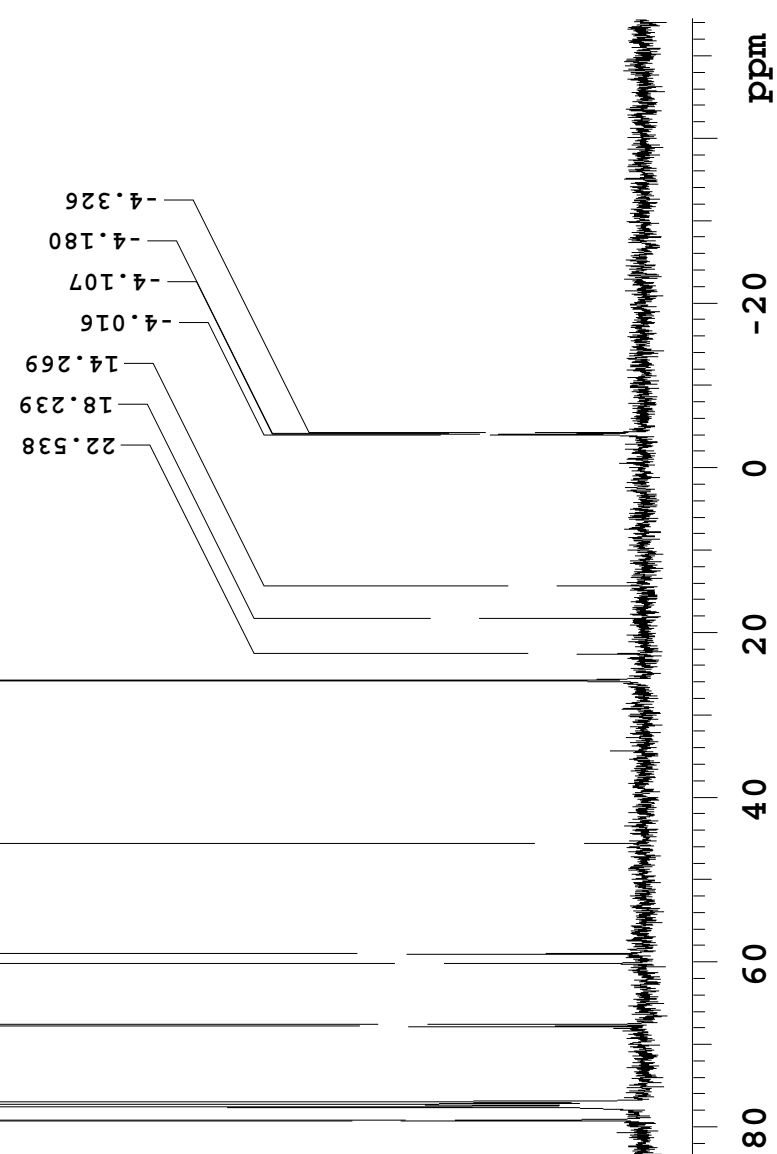

Q

ㄱ.

$\stackrel{\circ}{\sim}$

아

우

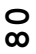

욱

$\stackrel{\circ}{\text { ก }}$

$867^{\circ} 8 z \mathrm{~T}$

$\varsigma \varepsilon L \cdot 8 Z T$

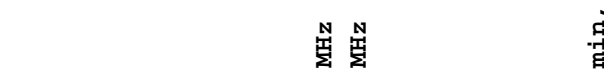

$\stackrel{\infty}{+}$

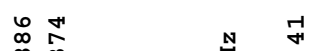

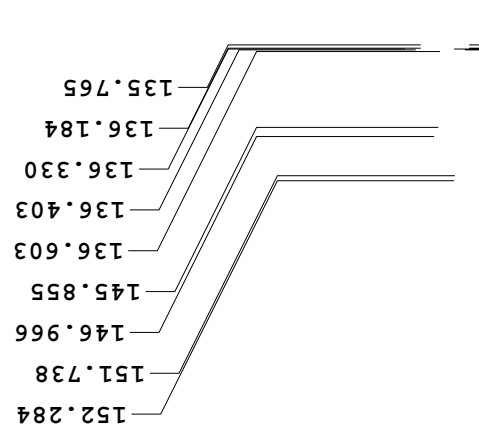

욱

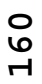


돔
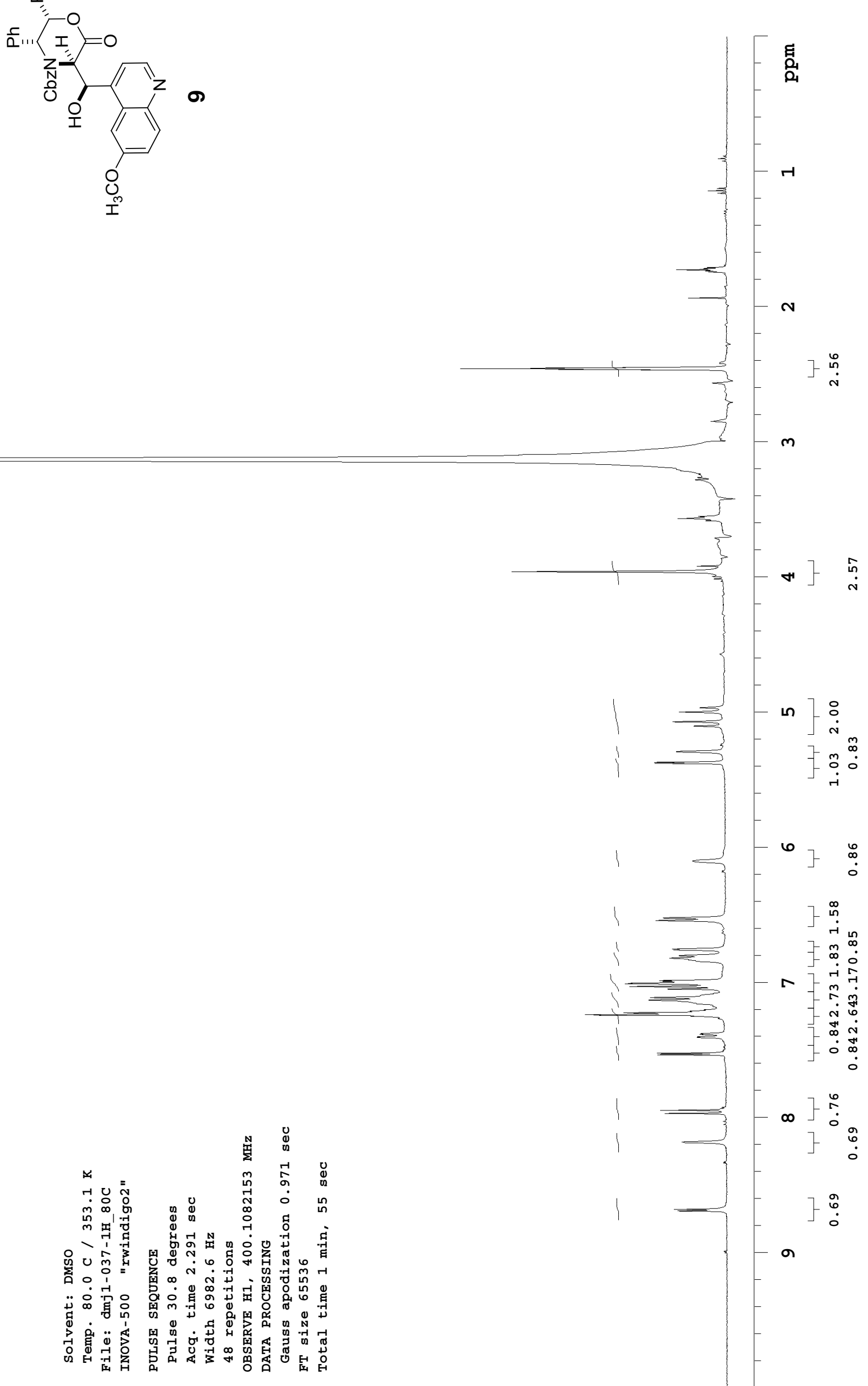
$\frac{5}{0}$
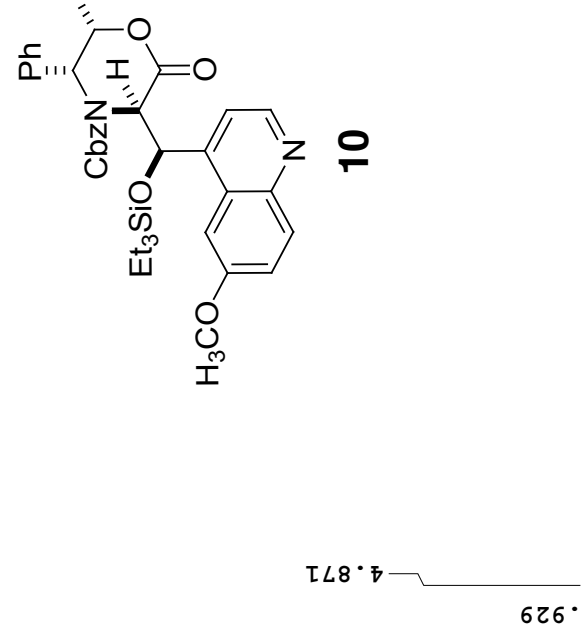

鬼
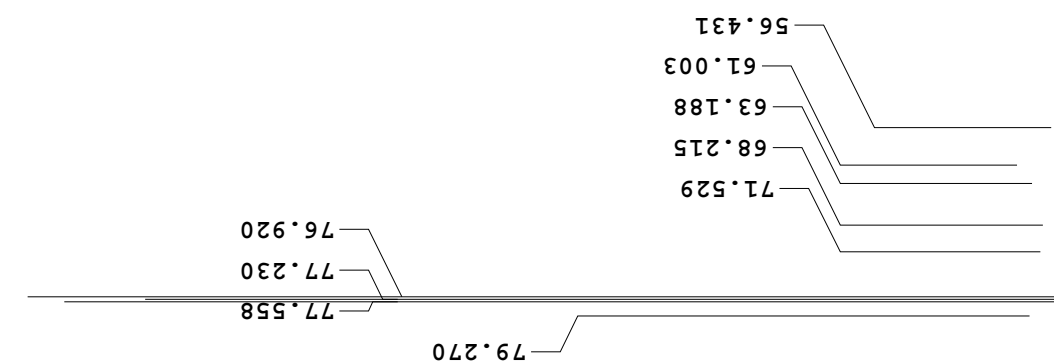

$\stackrel{\infty}{\infty}$

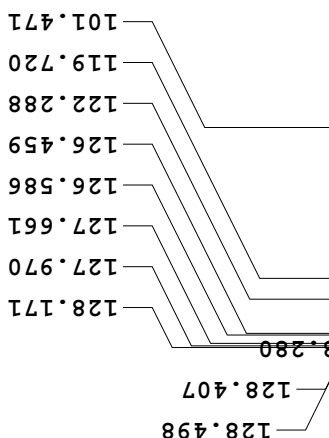


(1) 


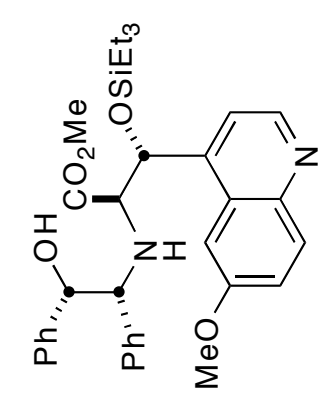

Page S39

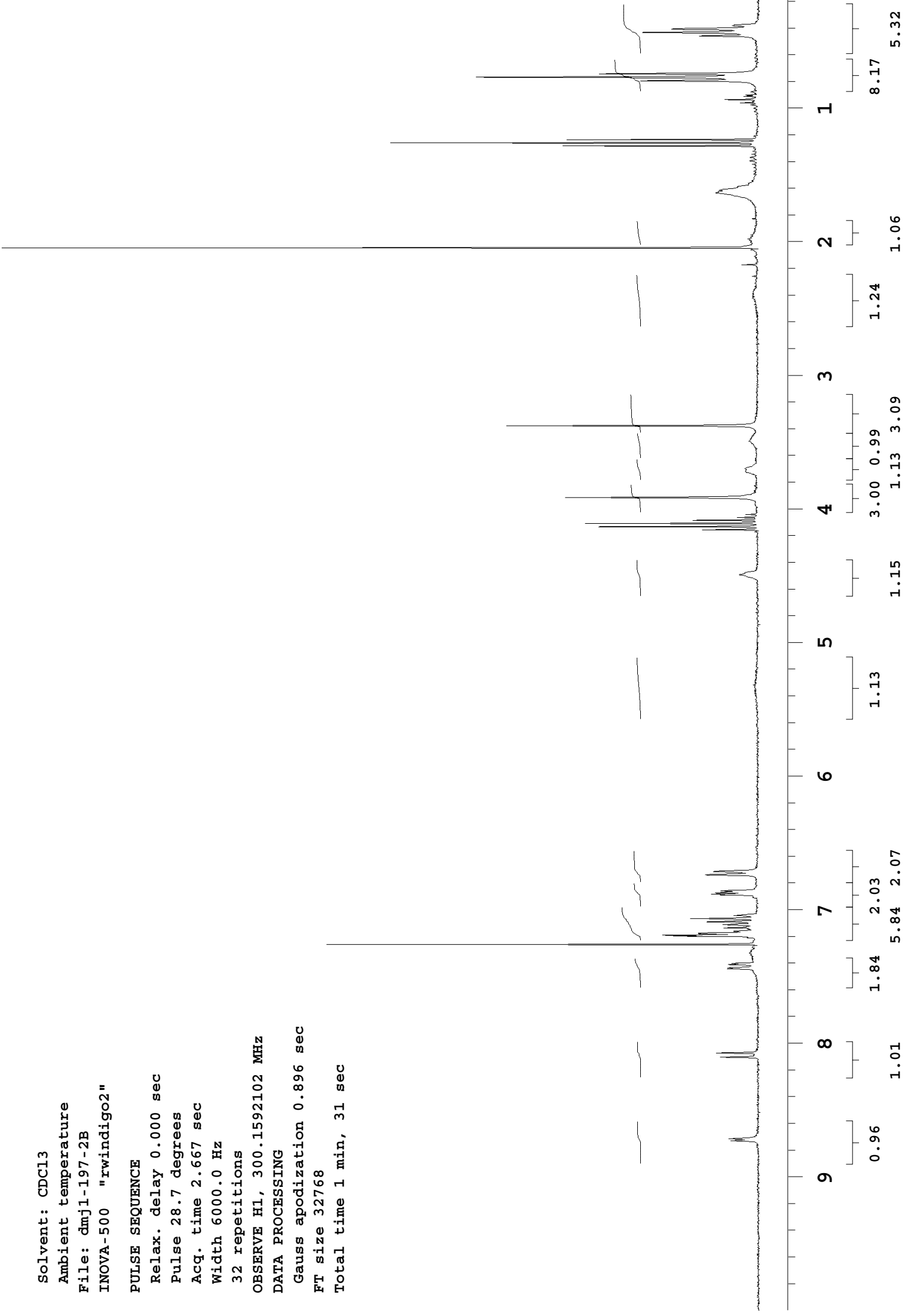




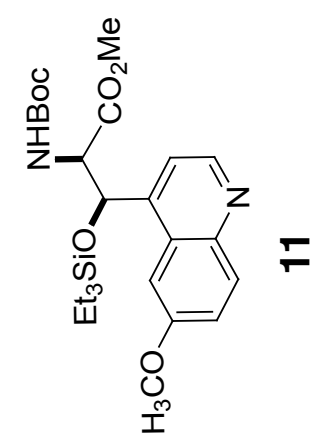




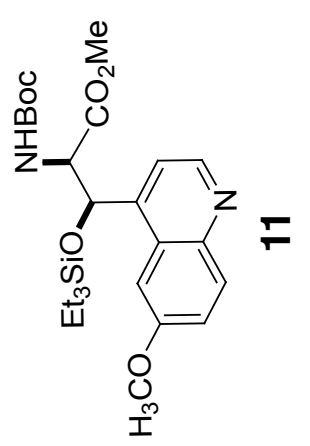

$688^{\circ} 9$

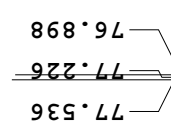

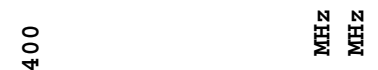

$8 \mathrm{ZL} \cdot \mathrm{TS}$

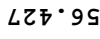

OST $6 S$

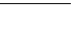

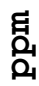

$\stackrel{\circ}{\text { 1 }}$

0

온

우

6

요

$6 \mp 2 \cdot 08$

욱

$Z Z S^{\circ} \tau 0 \mathrm{~T}$

$\angle 60.6 \tau I$

$\stackrel{\text { ㄱ }}{\text { ก }}$

† S. $9 Z \tau$

$00 L \cdot \tau \varepsilon \tau$

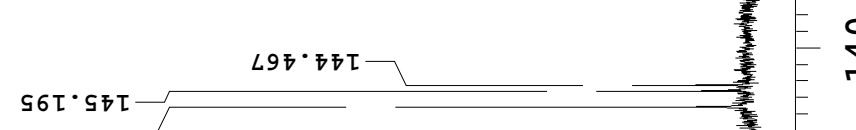

9 ZT・LTT

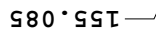

$60 \mathrm{~S} \cdot 8 \mathrm{SI}$

Ð $0 \cdot 69 \tau$

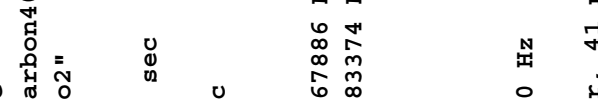

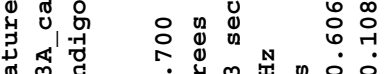

Tre

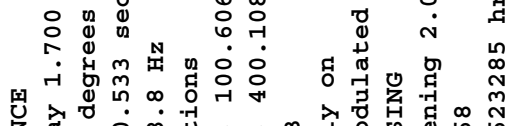

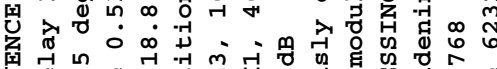

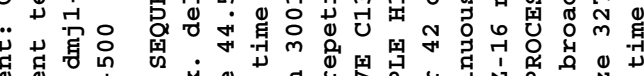

焉

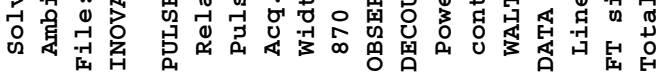




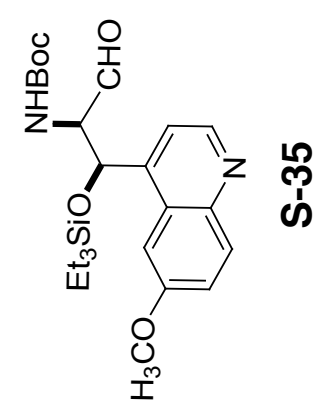

Page $\$ 42$

品

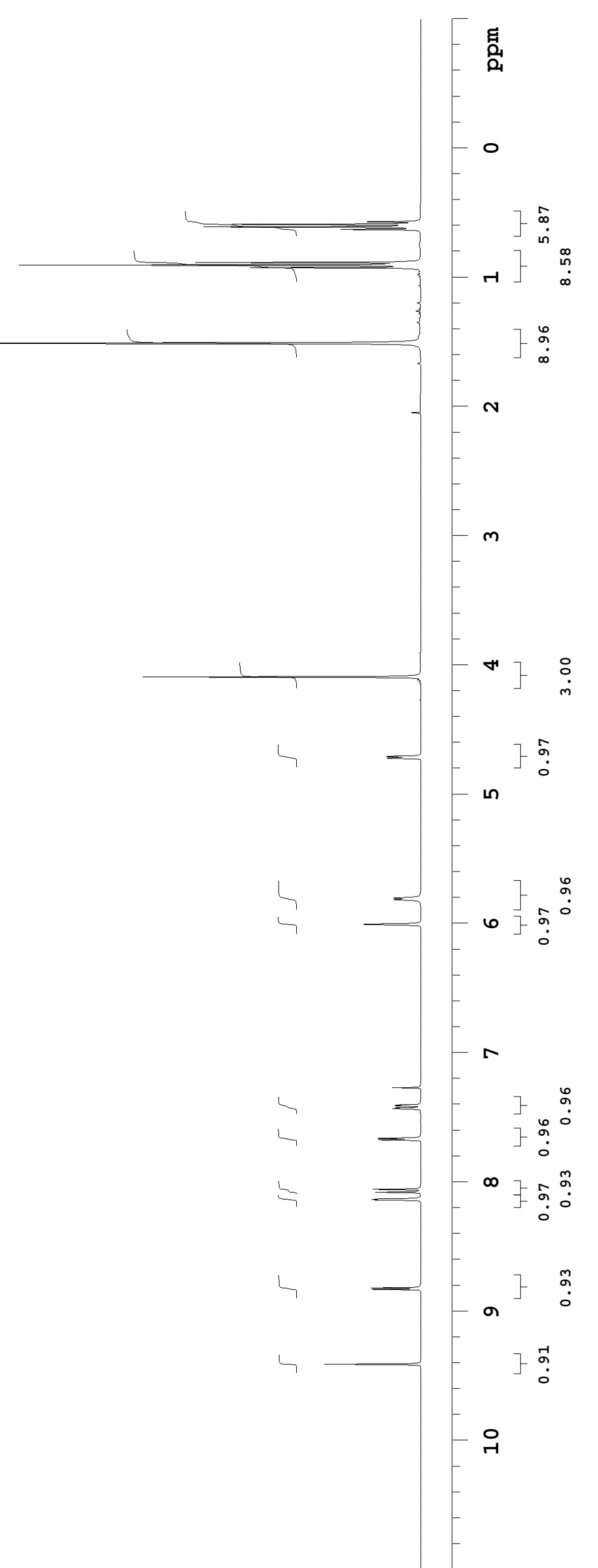




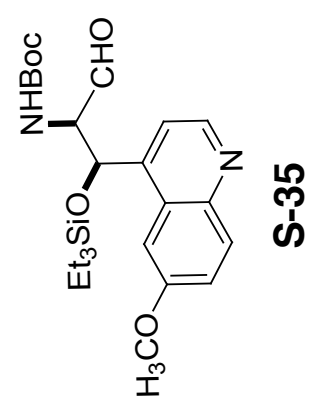

$\stackrel{1}{\circ}$

ฤฤし・

$\mathrm{S} \angle 8 \cdot 9$

온

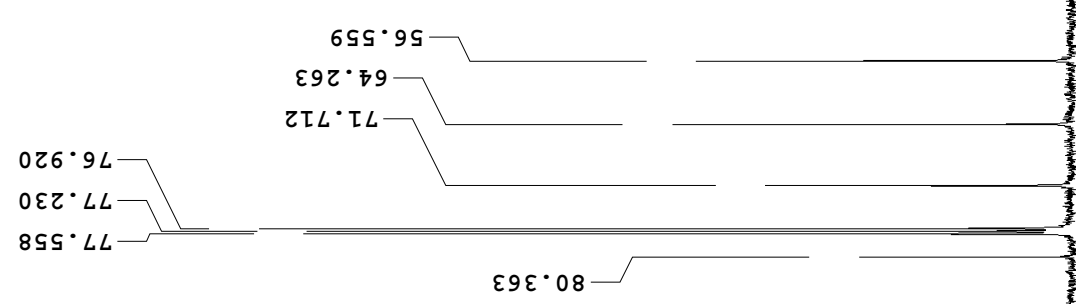

$6 \mathrm{~s} \cdot 9 \mathrm{~s}$

6

$\infty$

움

$878 \cdot 8 \tau T$

$\stackrel{\circ}{\text { ก }}$

$0 S S \cdot 9 Z \mathrm{~L}$

$0 \varsigma 8^{\circ} \tau \varepsilon \tau$

$\stackrel{\text { 감 }}{\circ}$

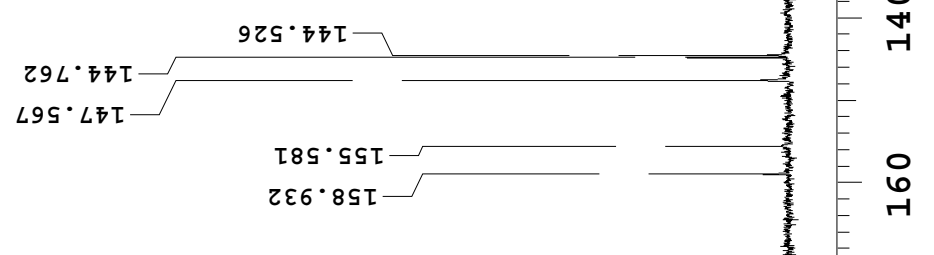

变函

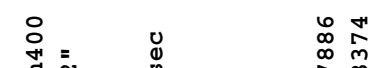

N652

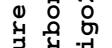

o

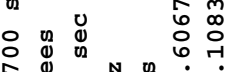

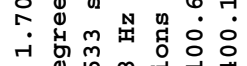

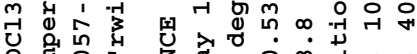

ช) $\stackrel{\dot{n}}{\dot{4}}$

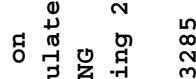

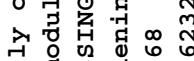

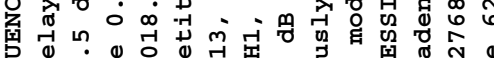

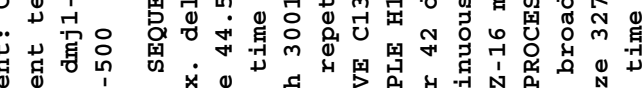

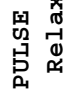

N 


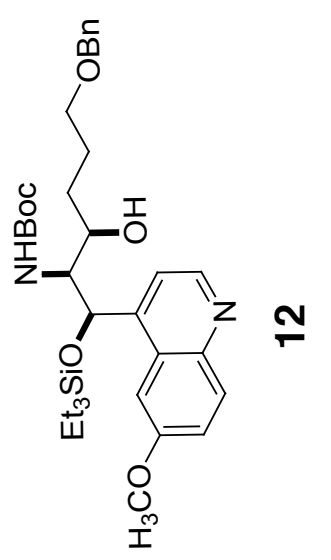

Page S44

吕

。

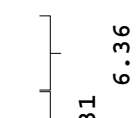

- $1-9$

$\boldsymbol{N}^{-}$

$m$

\}

ᄀ

H

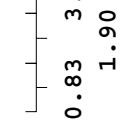

เก

] ั̊

o] 范

N $\begin{gathered}0 \\ 0 \\ 0 \\ 0 \\ 0\end{gathered}$

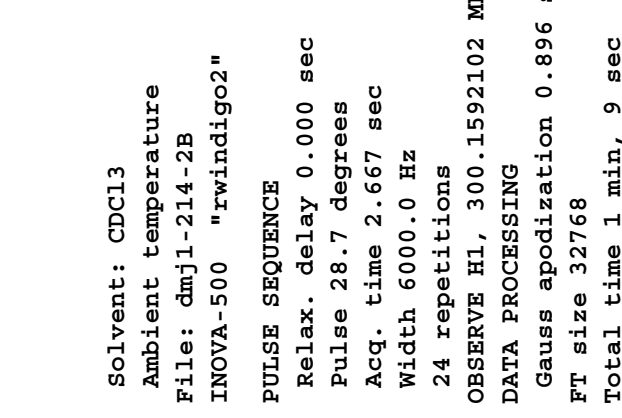

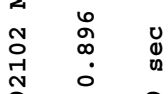

둔

急

궁 

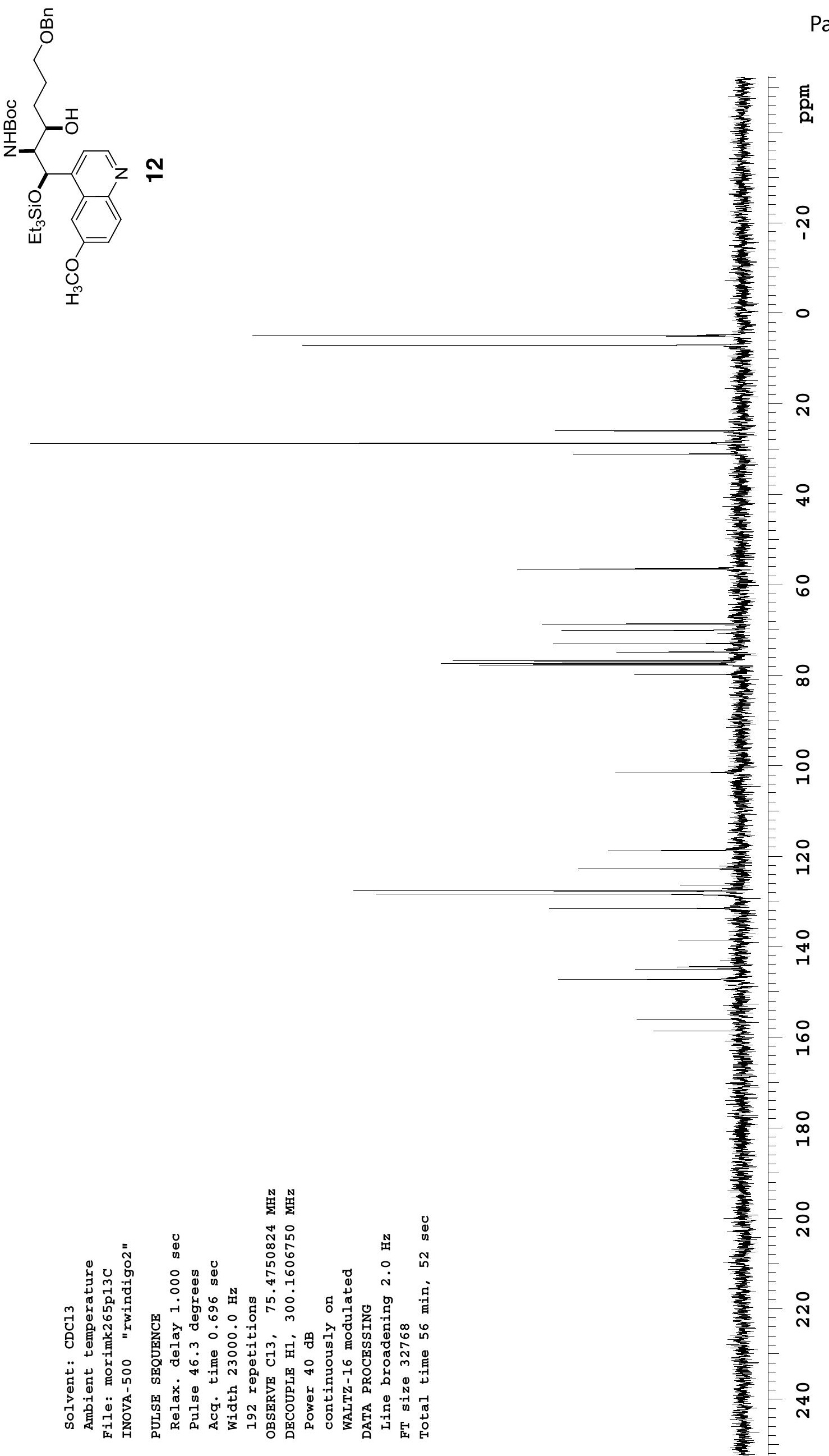

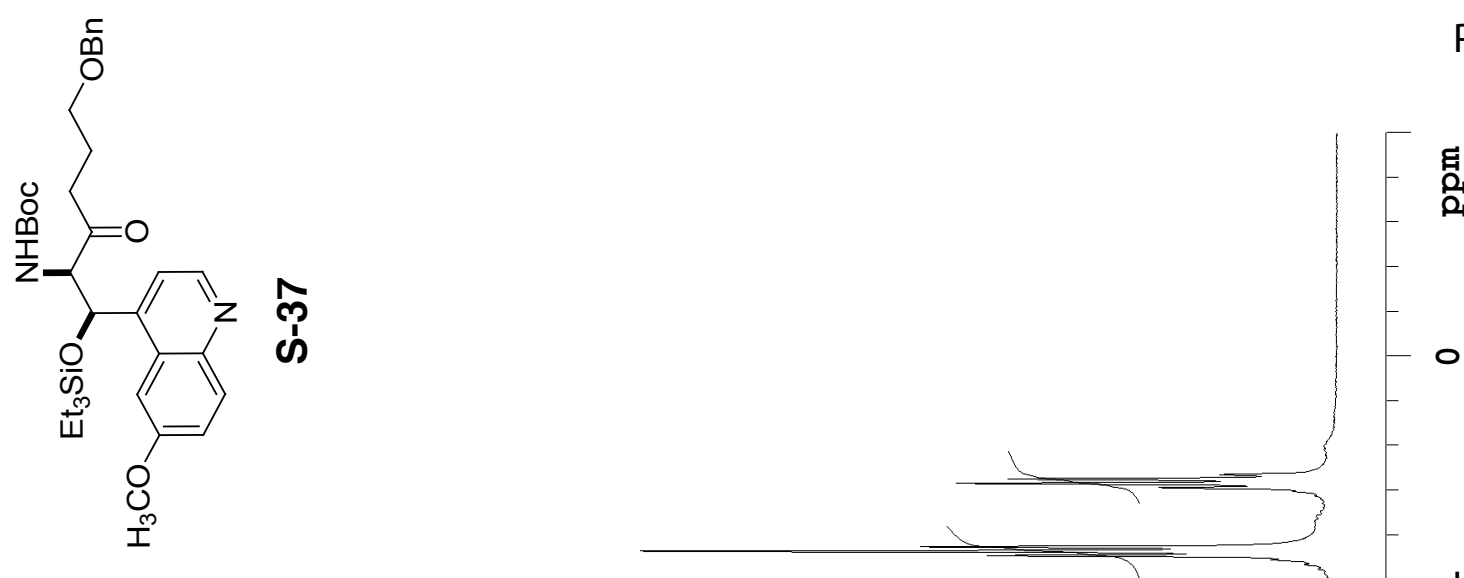

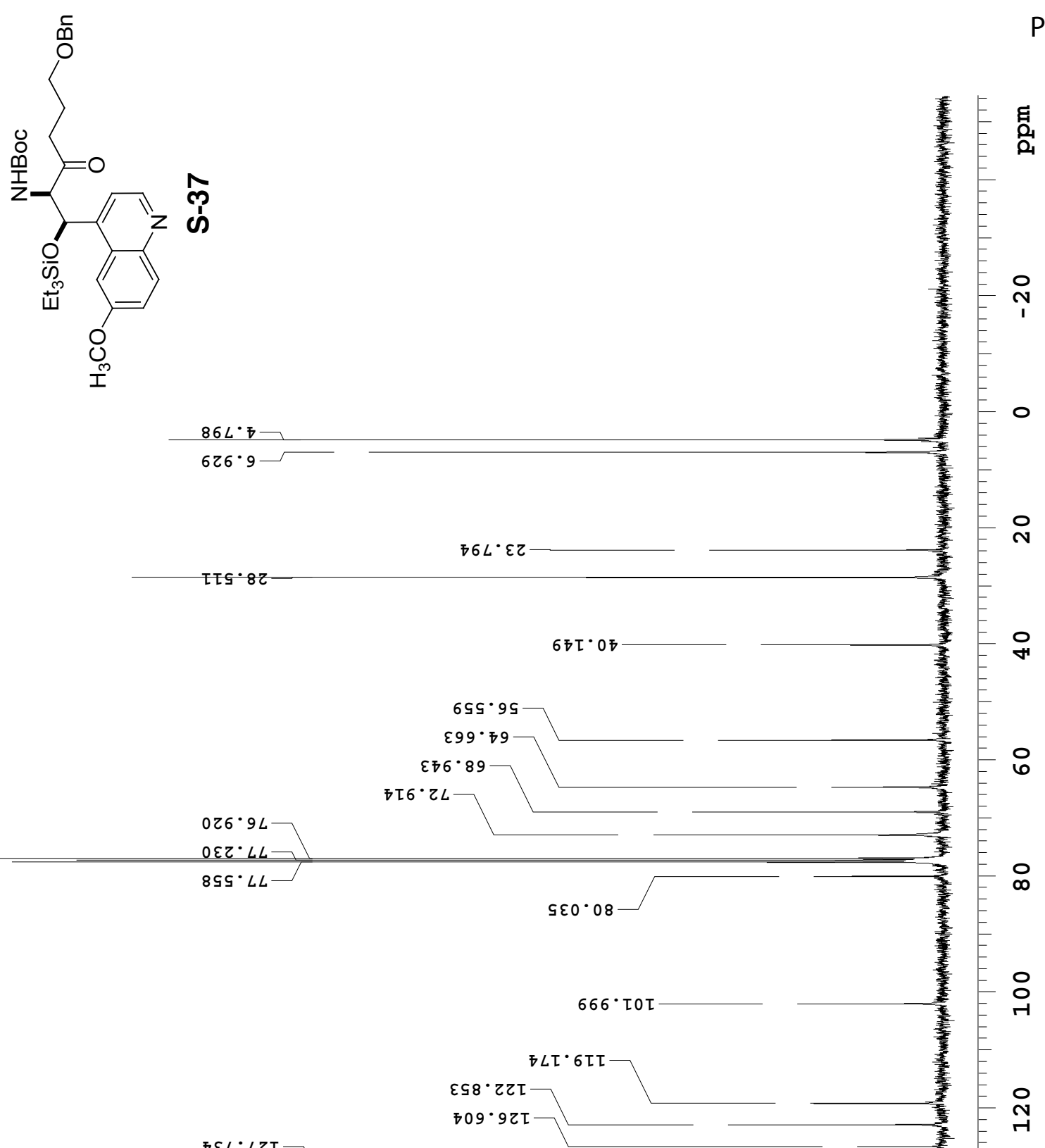

$\varpi \varepsilon L^{\cdot} L Z \tau$ ६૬ऽ· $8 乙 \tau$

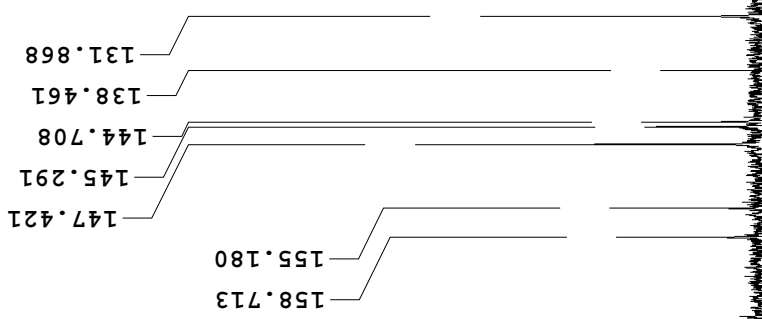



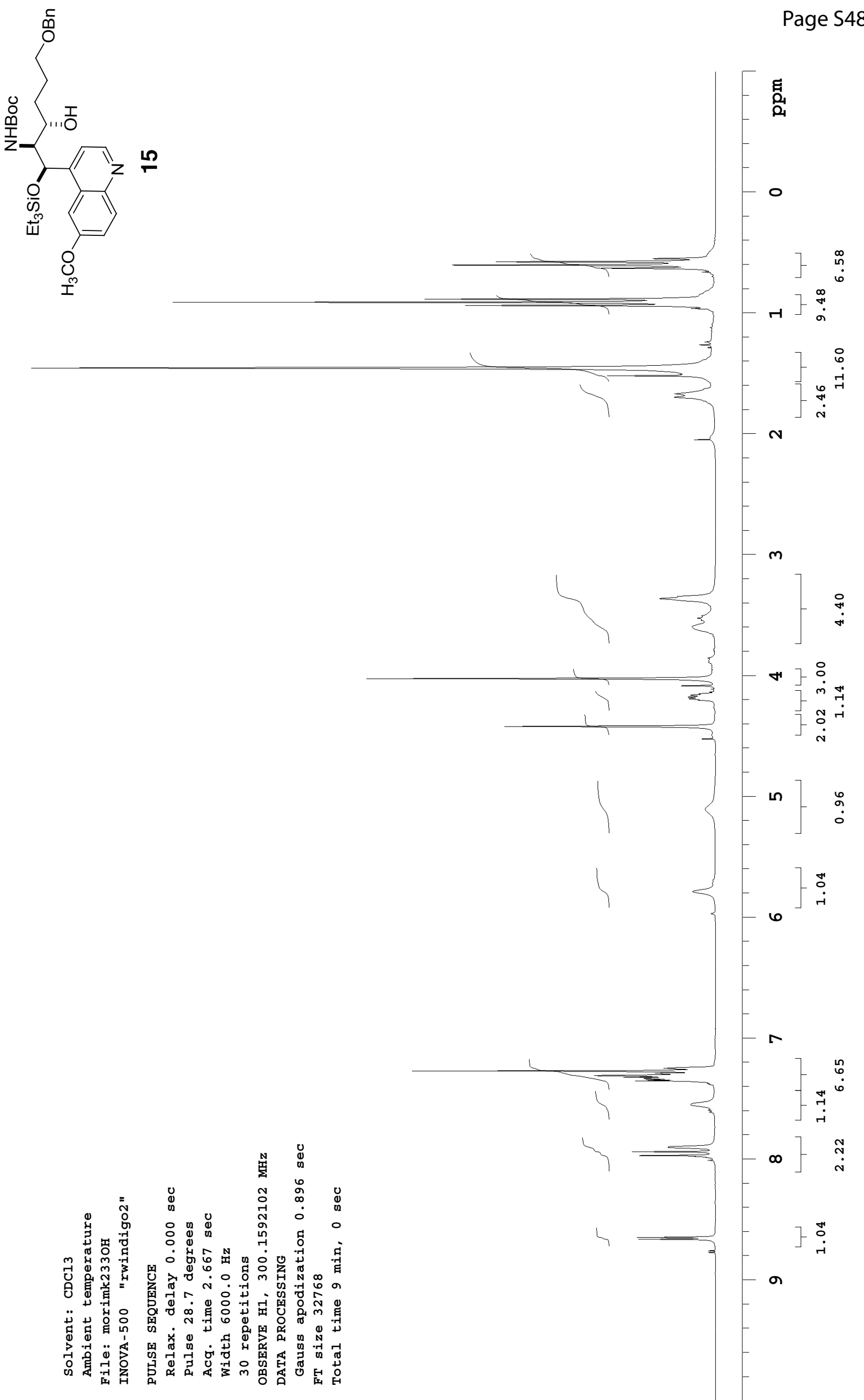
Page S49

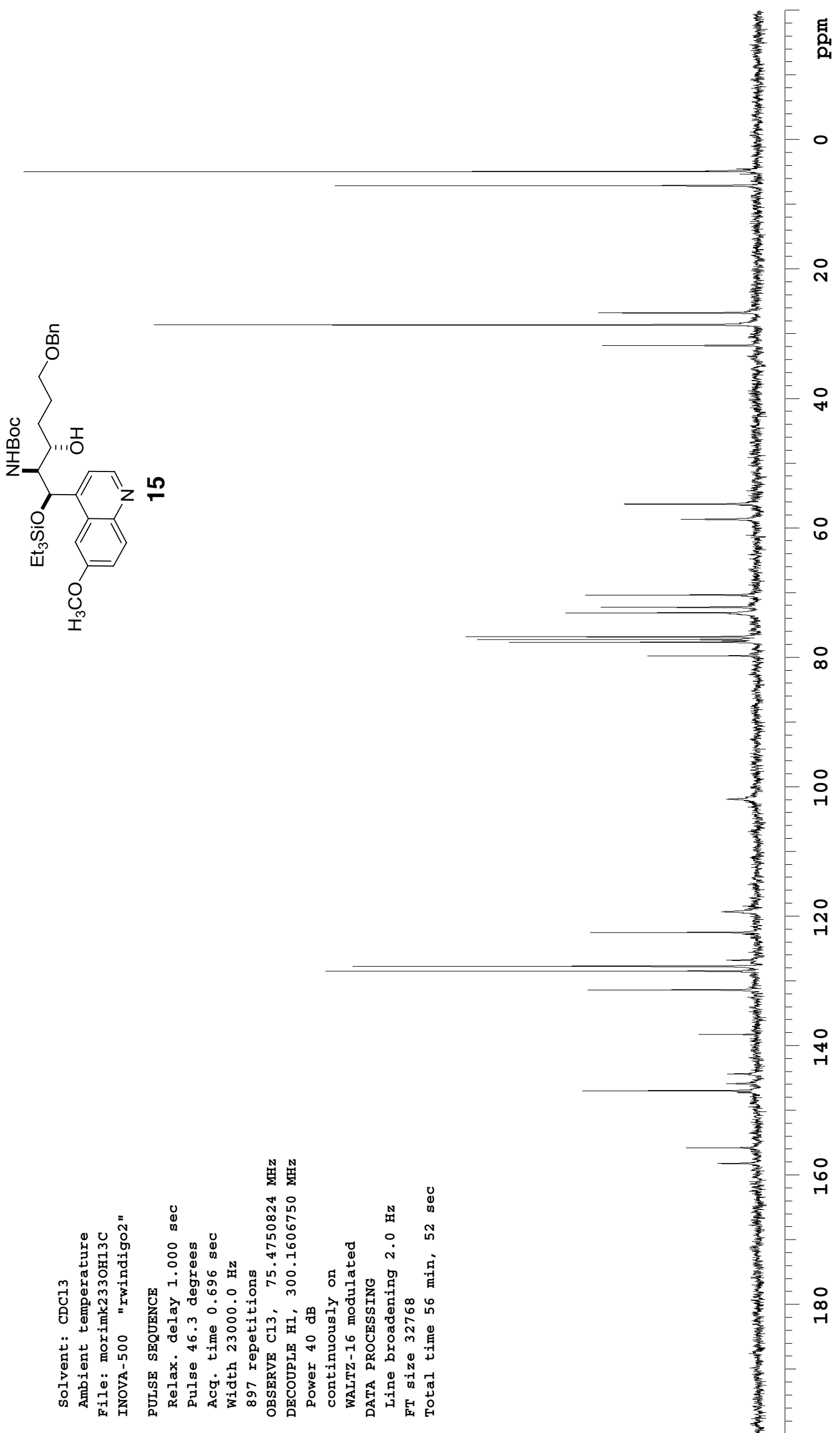




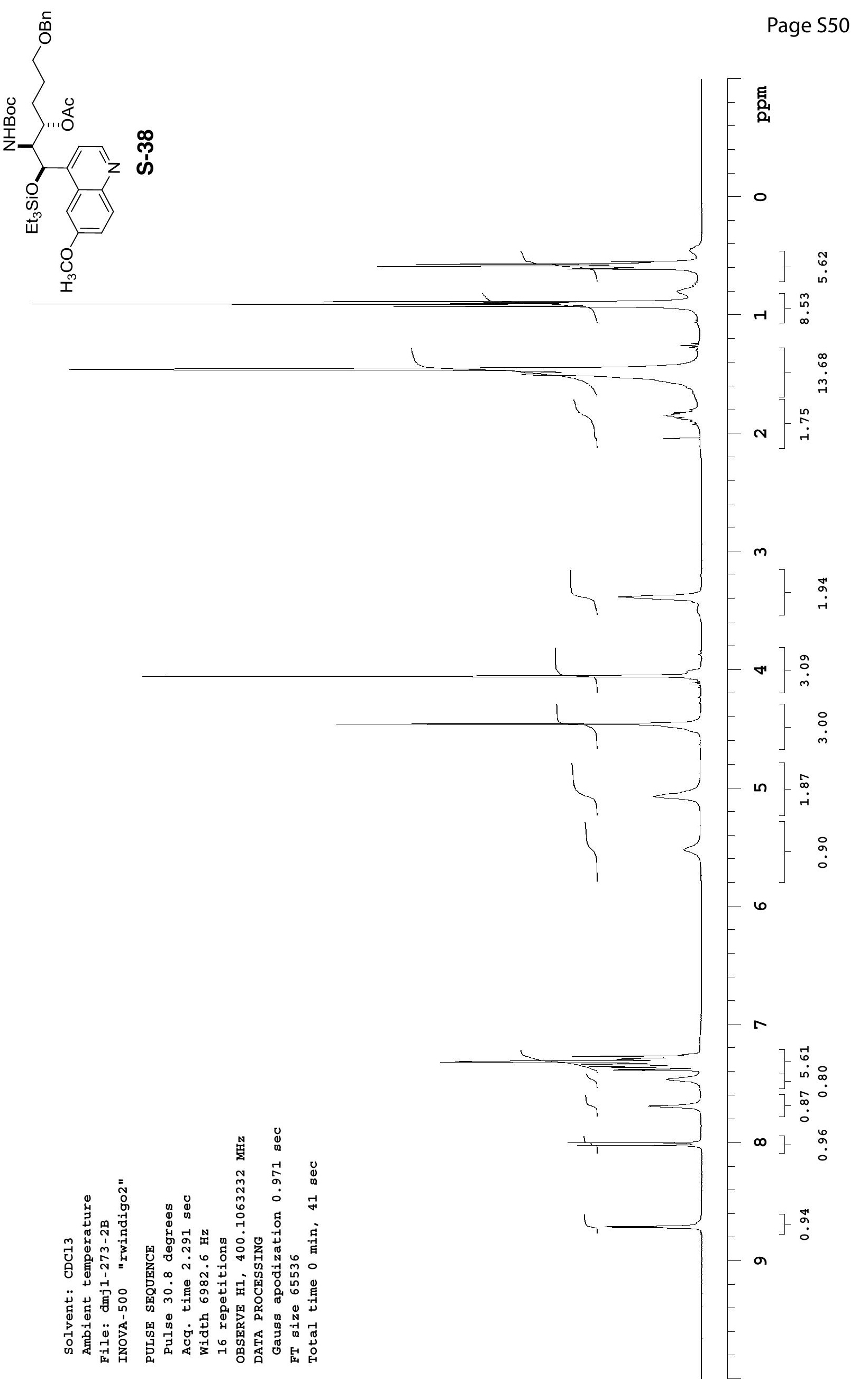




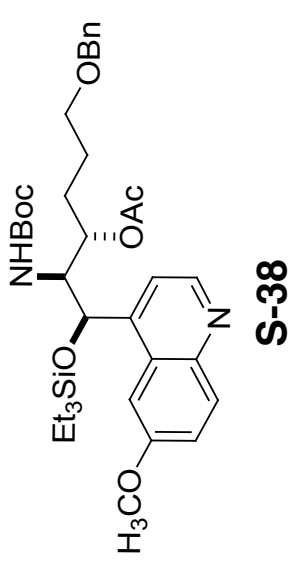

ڤ్

$\angle[8 \cdot 7 \longrightarrow$

$586^{\circ} 9$

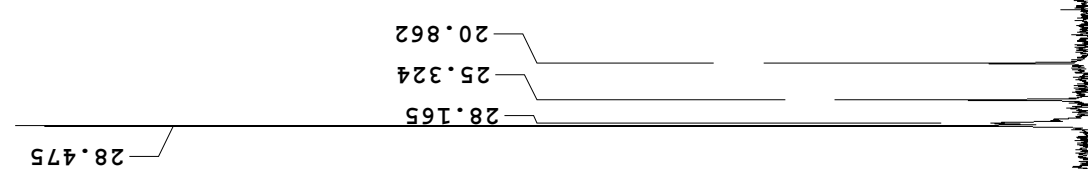

우

우

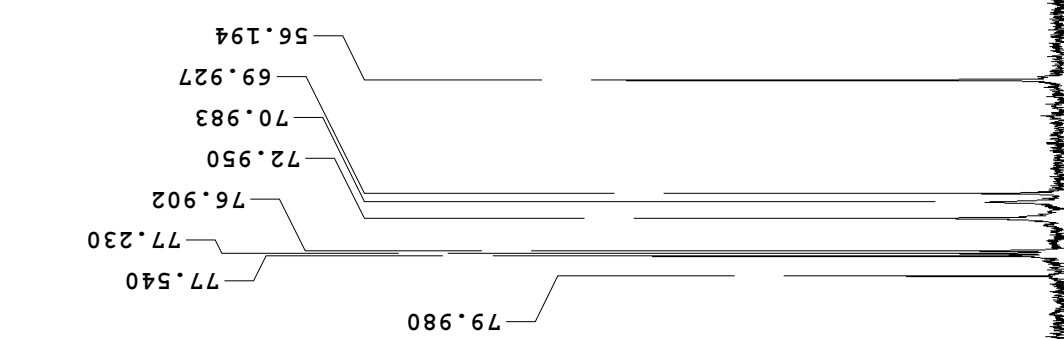

6

$\stackrel{\infty}{\infty}$

욱

$\stackrel{\circ}{7}$

$\varpi \varepsilon L \cdot L \tau \tau$

$L \tau G \cdot 8 Z \tau$

䨔紫

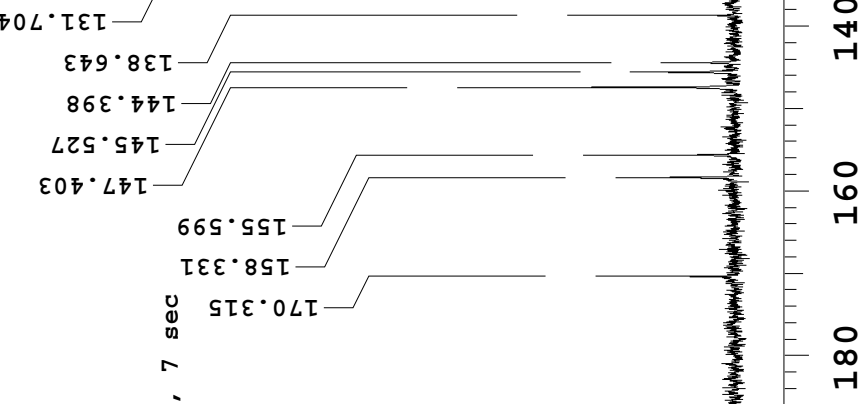

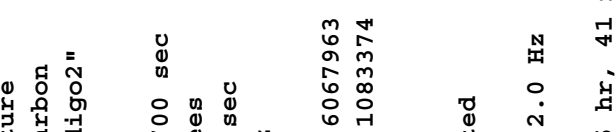

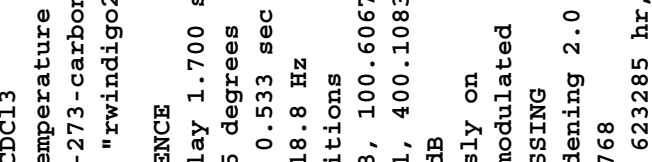

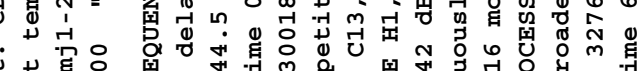

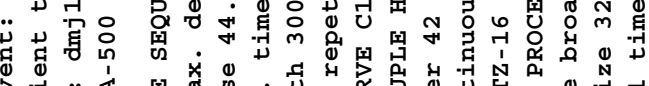

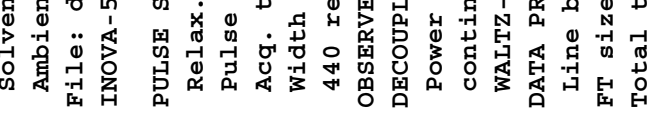




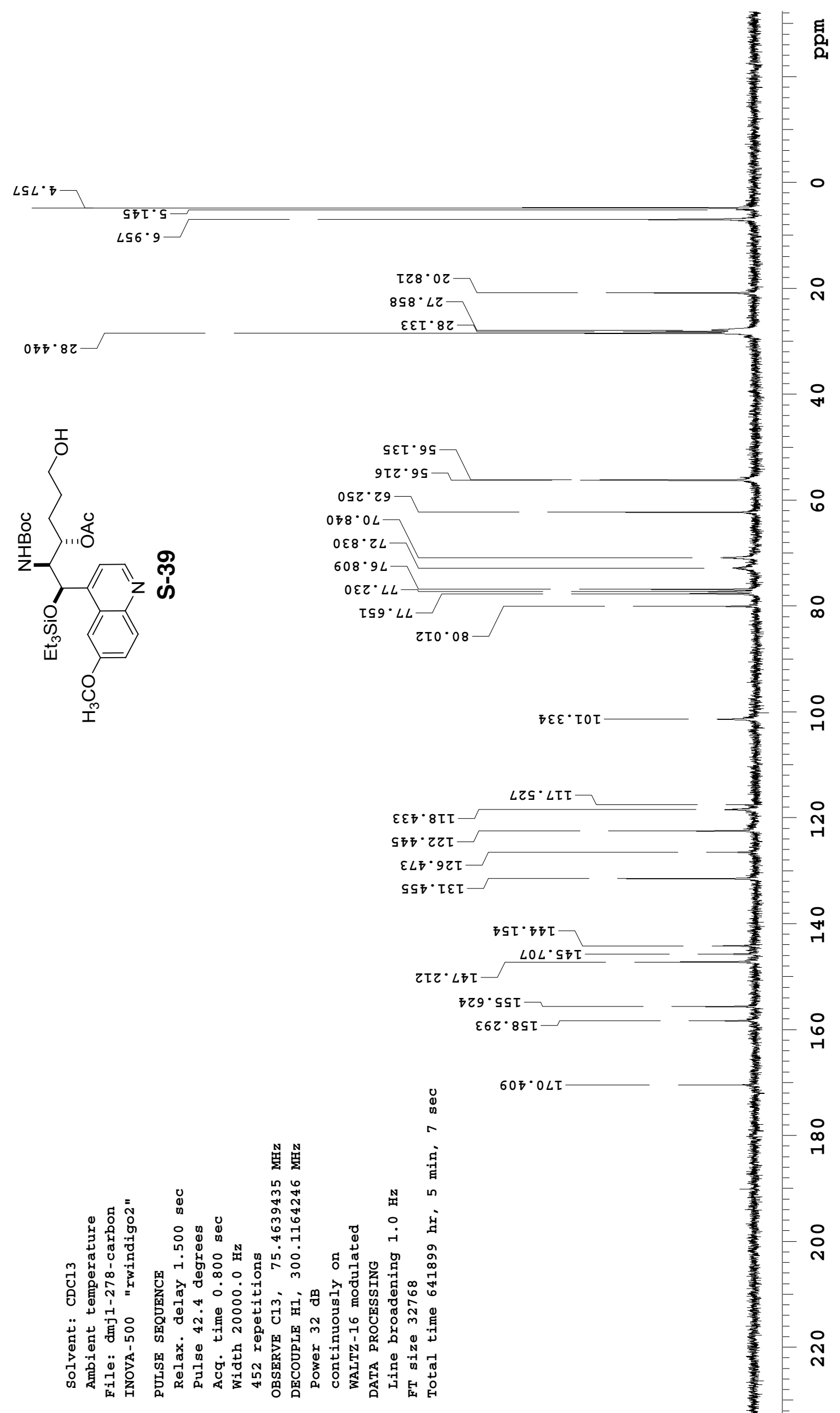




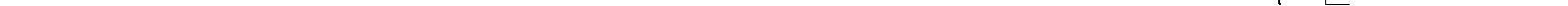



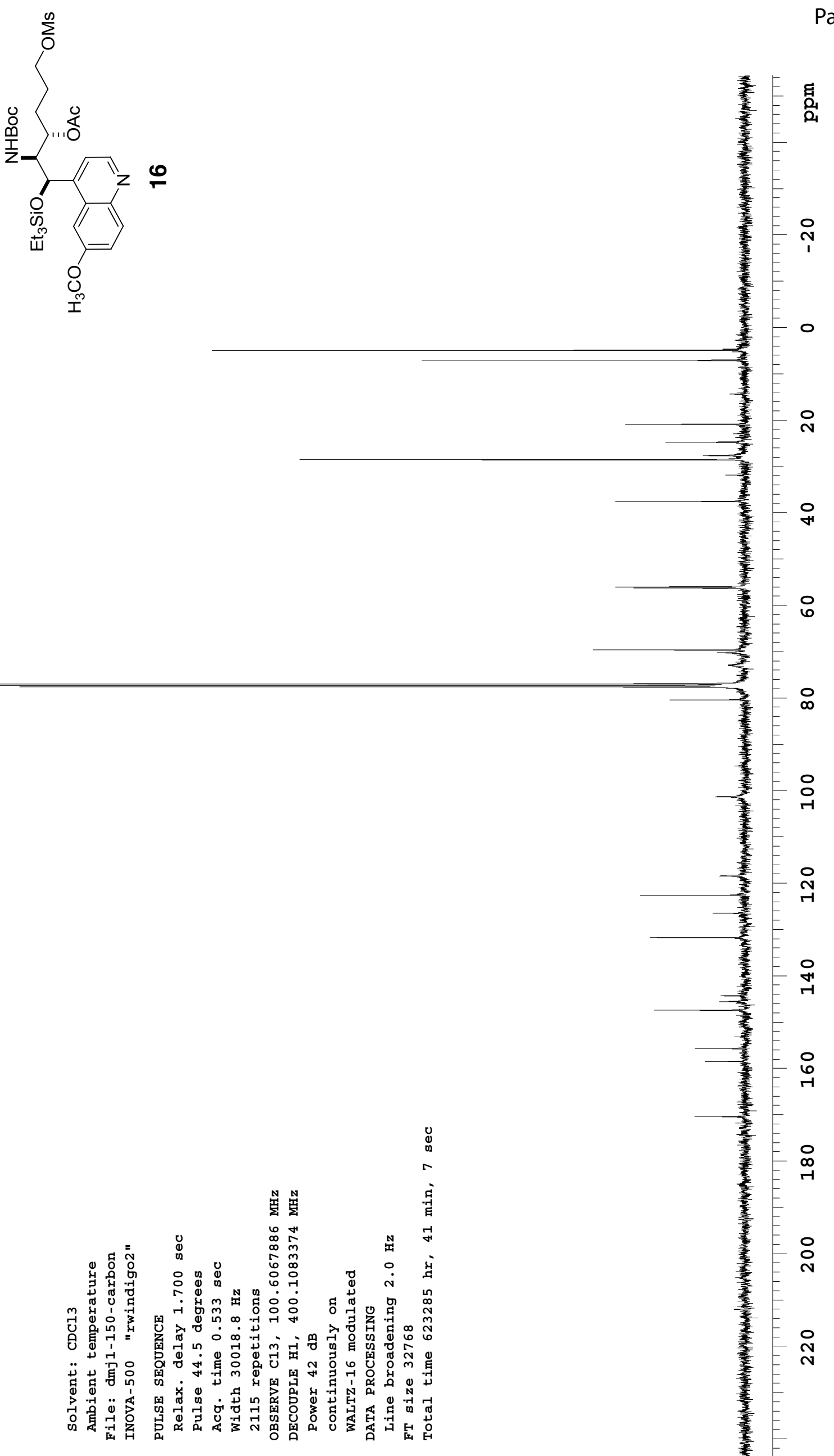


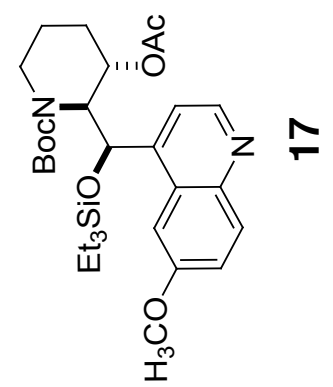




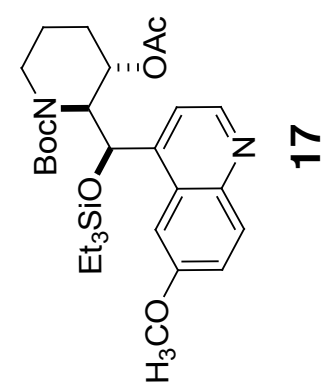

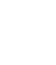


Page S58
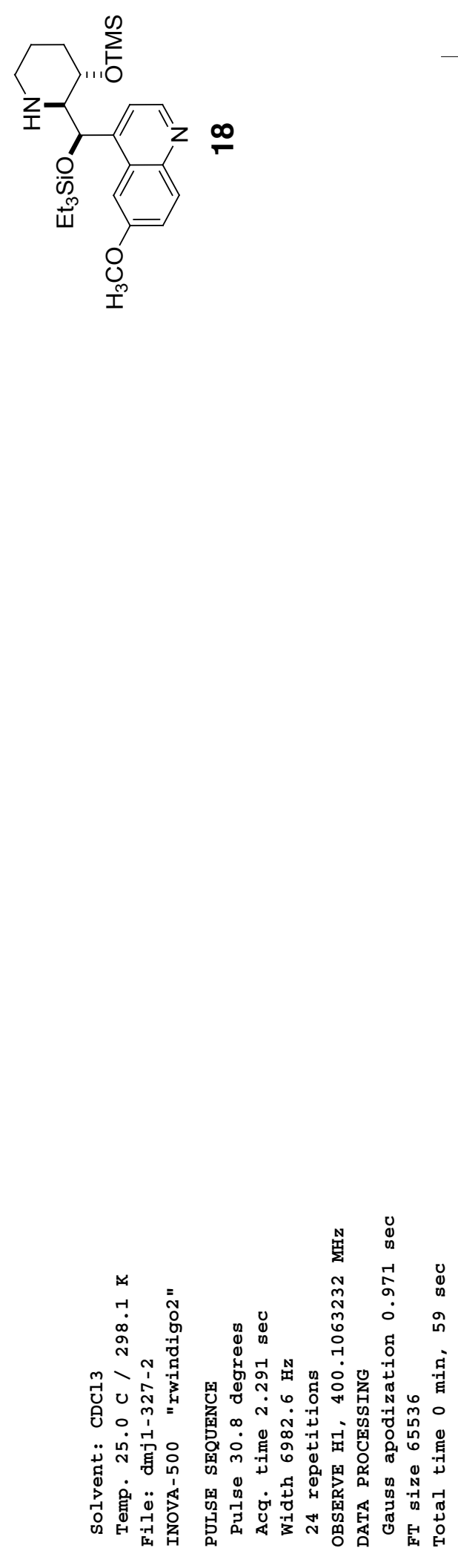

हू
مू

○」 กัก

- $\begin{array}{r}6 \\ \text { กे }\end{array}$

$+] \stackrel{m}{a}$

4

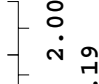

- ล

N] $\begin{aligned} & 6 \\ & -\end{aligned}$

$\int \stackrel{\text { ก }}{\text { r }}$

$m] \quad \stackrel{0}{\dot{0}}$

F

rJ

ก

$\checkmark \stackrel{\infty}{\circ}:$

6

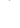

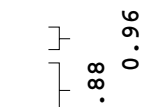

$\infty] \quad \begin{aligned} & -1 \\ & 0\end{aligned}$

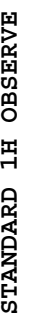




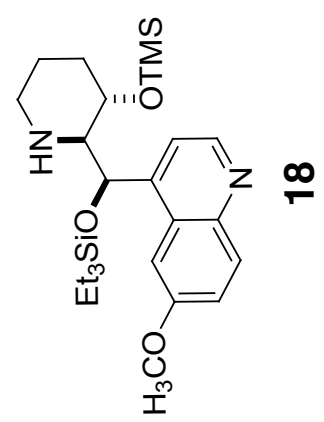

복

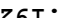

$L \mathrm{LO} \cdot \mathrm{s}$

$80 \varepsilon^{\circ} \mathrm{s}$

$200 . \mathrm{L}$

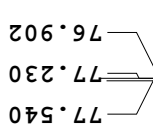
$6 \varpi G \cdot L \succsim \tau$

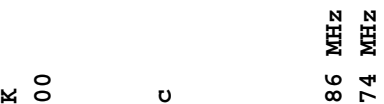

$99 L^{\cdot} \varepsilon 0 \tau$

ㄷ. 若 స

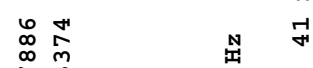

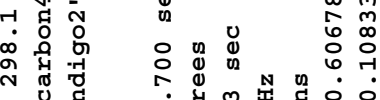

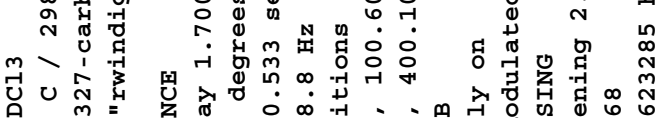

色

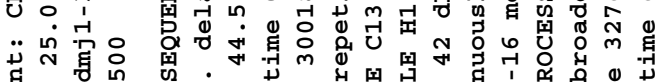

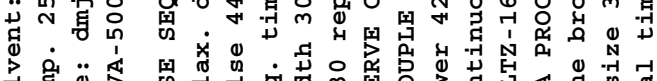



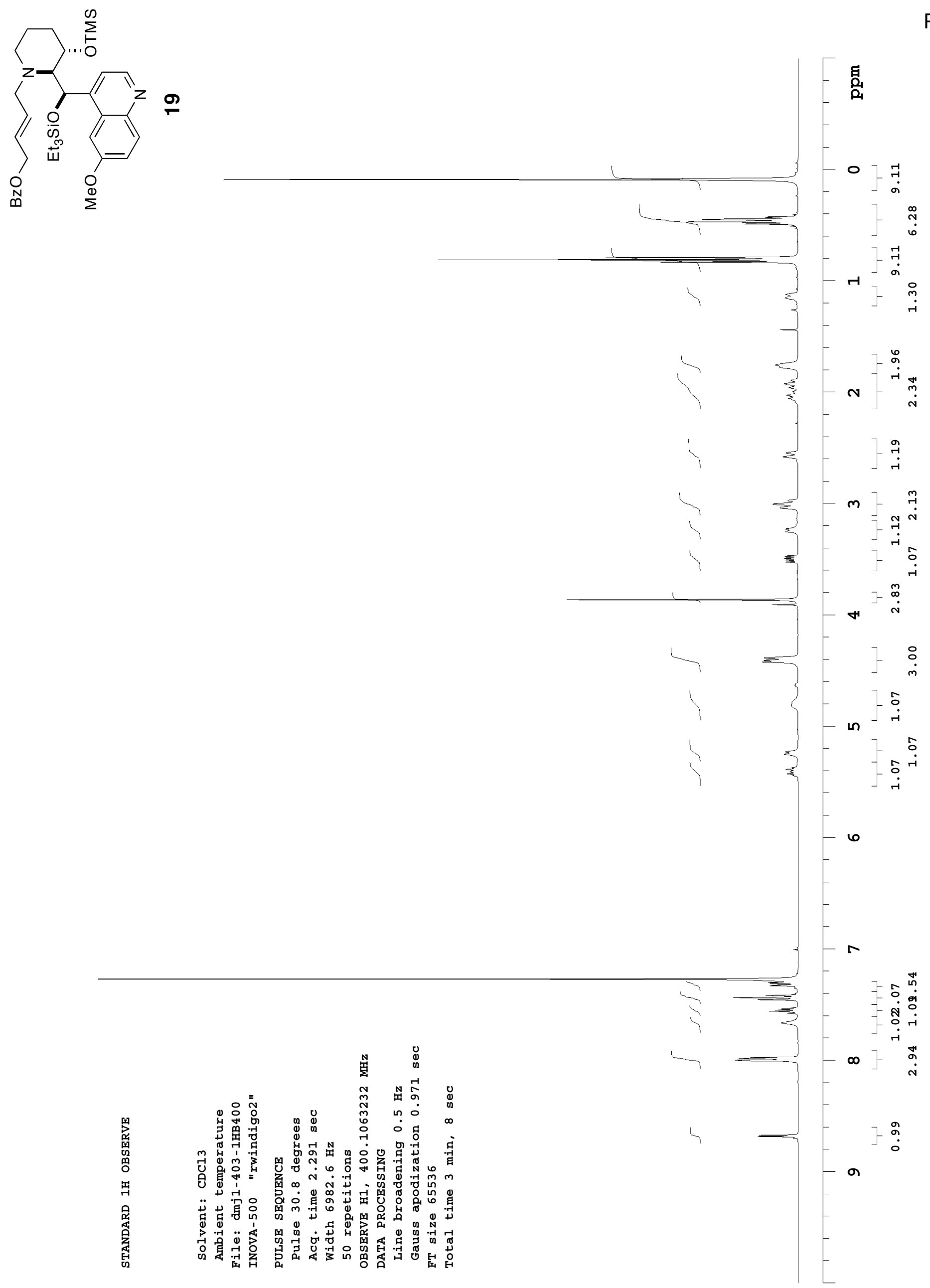

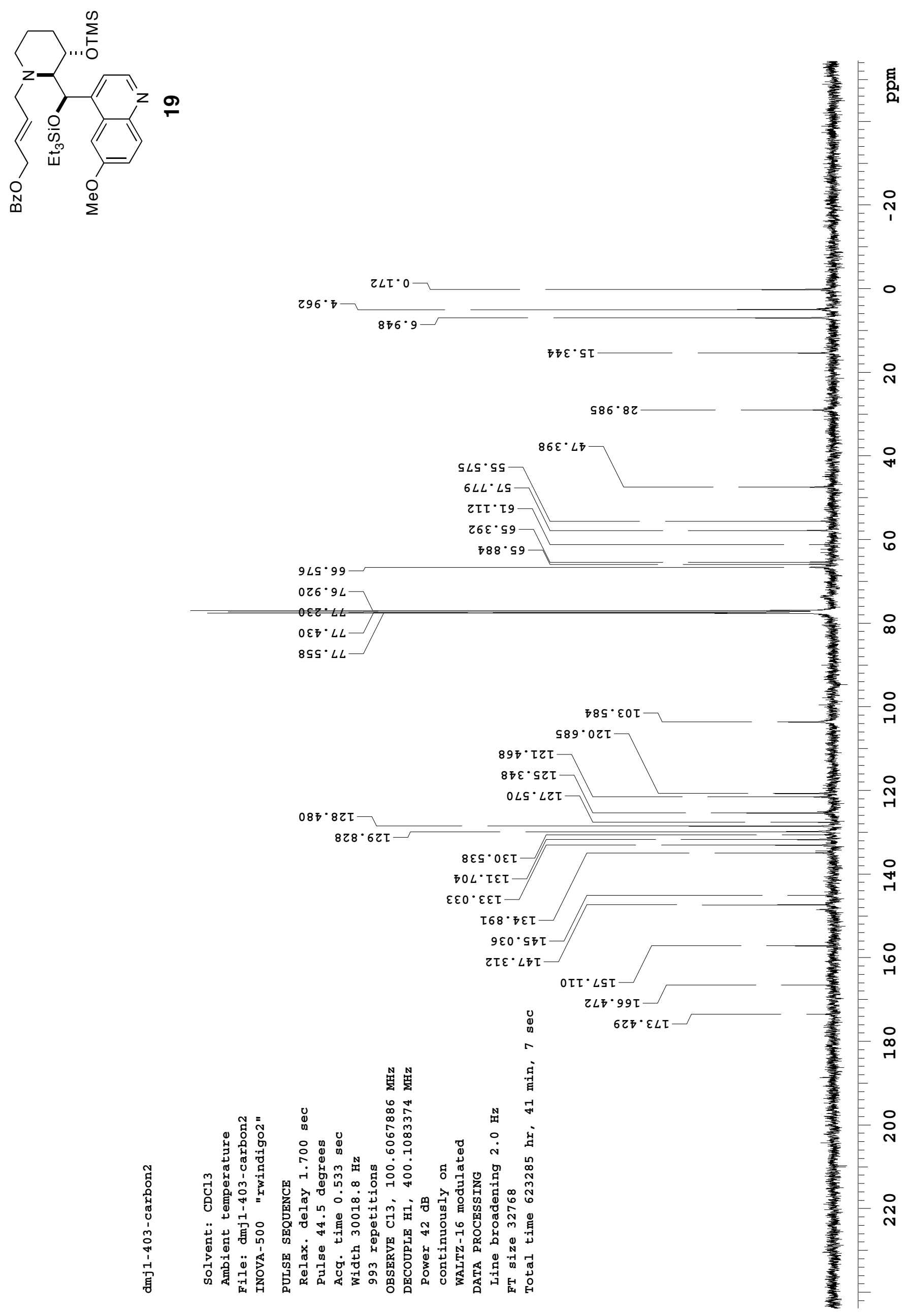
Page S62

$\sum_{\substack{0 \\ \infty}} \bar{j}$

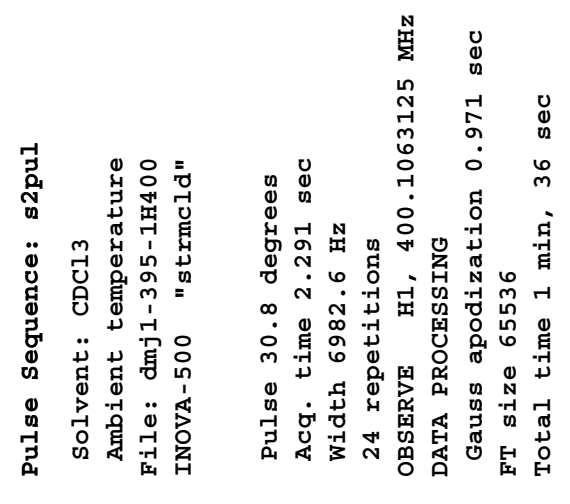



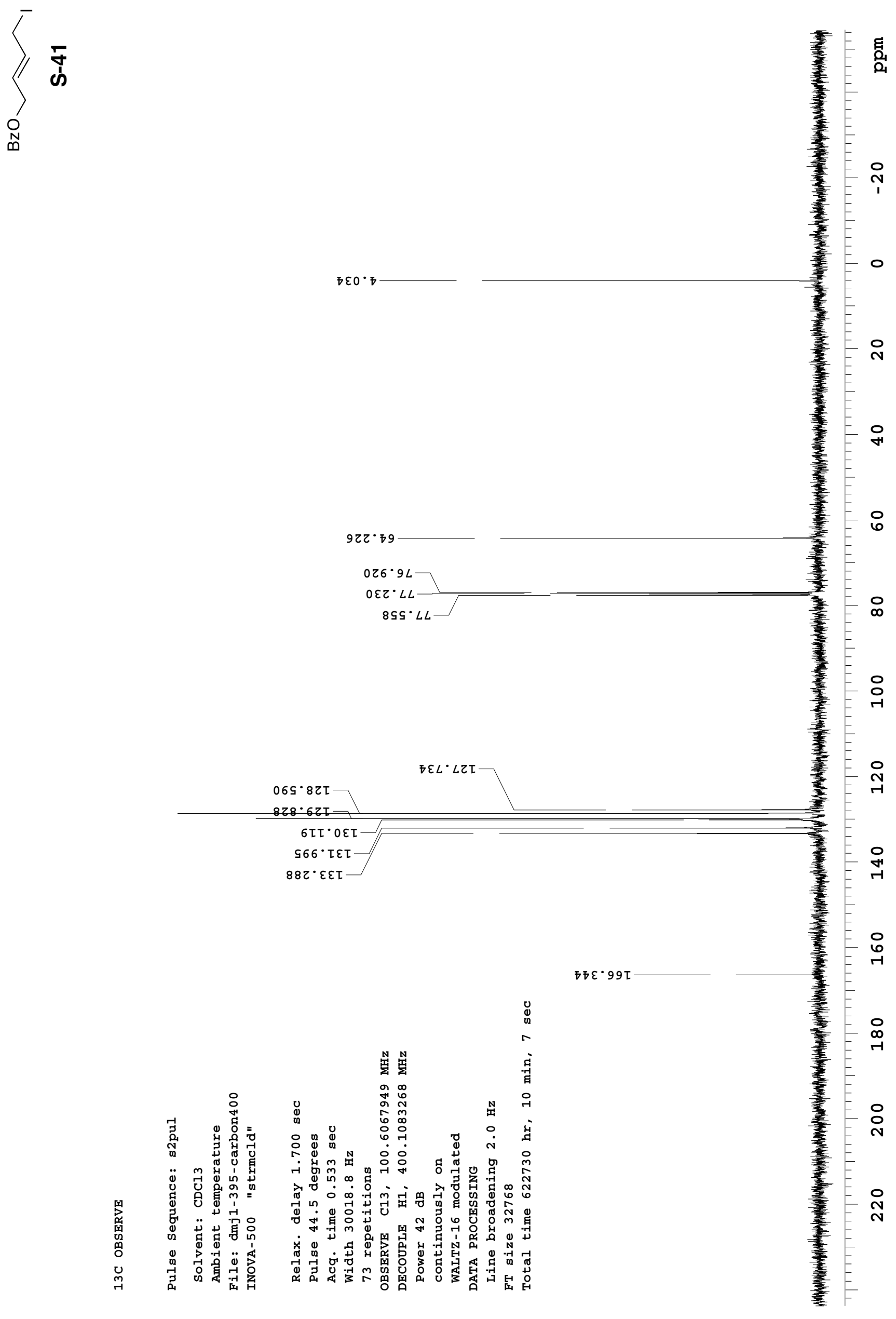


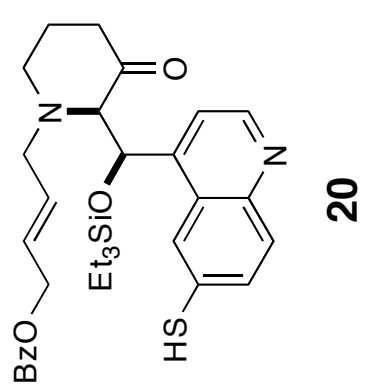

Page S64

뎡

o

- $\begin{aligned} & \infty \\ & \stackrel{5}{0}\end{aligned}$

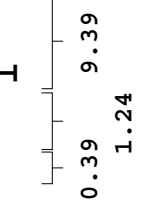

N]

ติ.

(1)

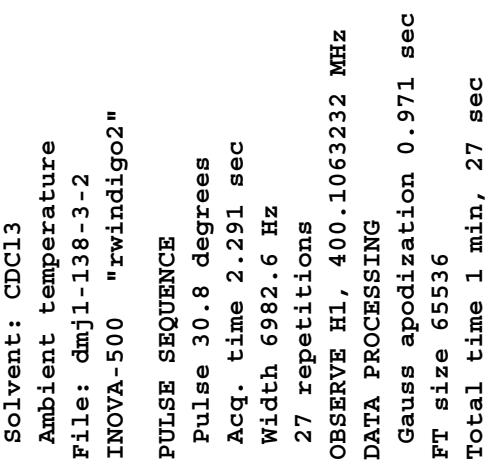

เก

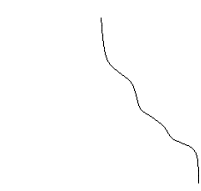

$-\quad \frac{\cos }{\text { i }}$

6

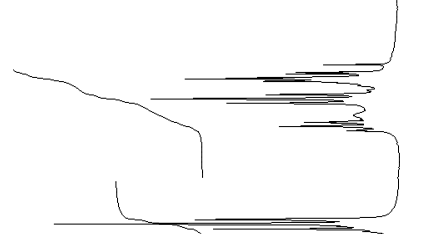

官

$\infty \underset{+}{\stackrel{H}{n}}$
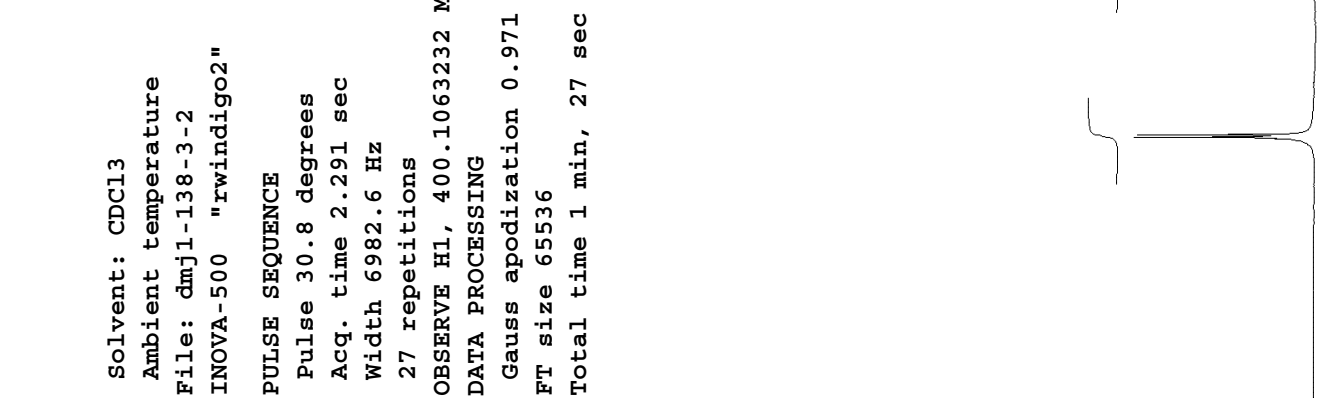

i 
$\begin{array}{ll}I=0 & \text { Page S65 }\end{array}$

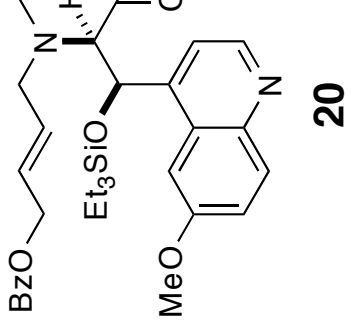

: 


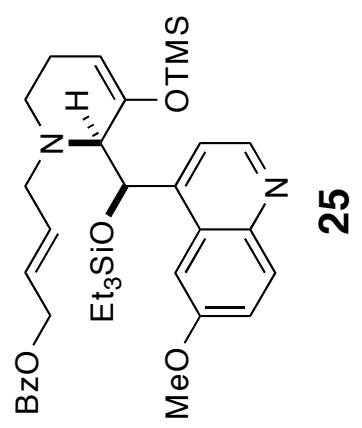

్ㅗㅇ

0

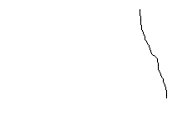

N $\begin{gathered}0 \\ 0 \\ 0\end{gathered}$

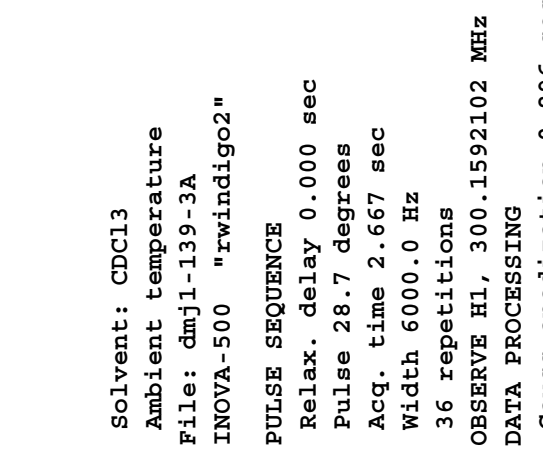




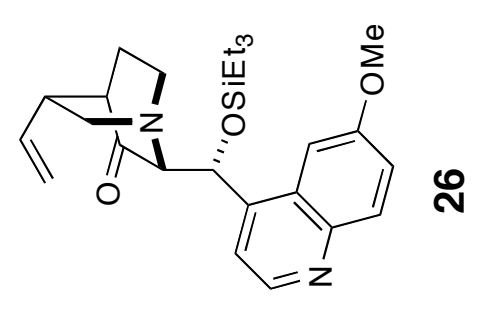

Page S67

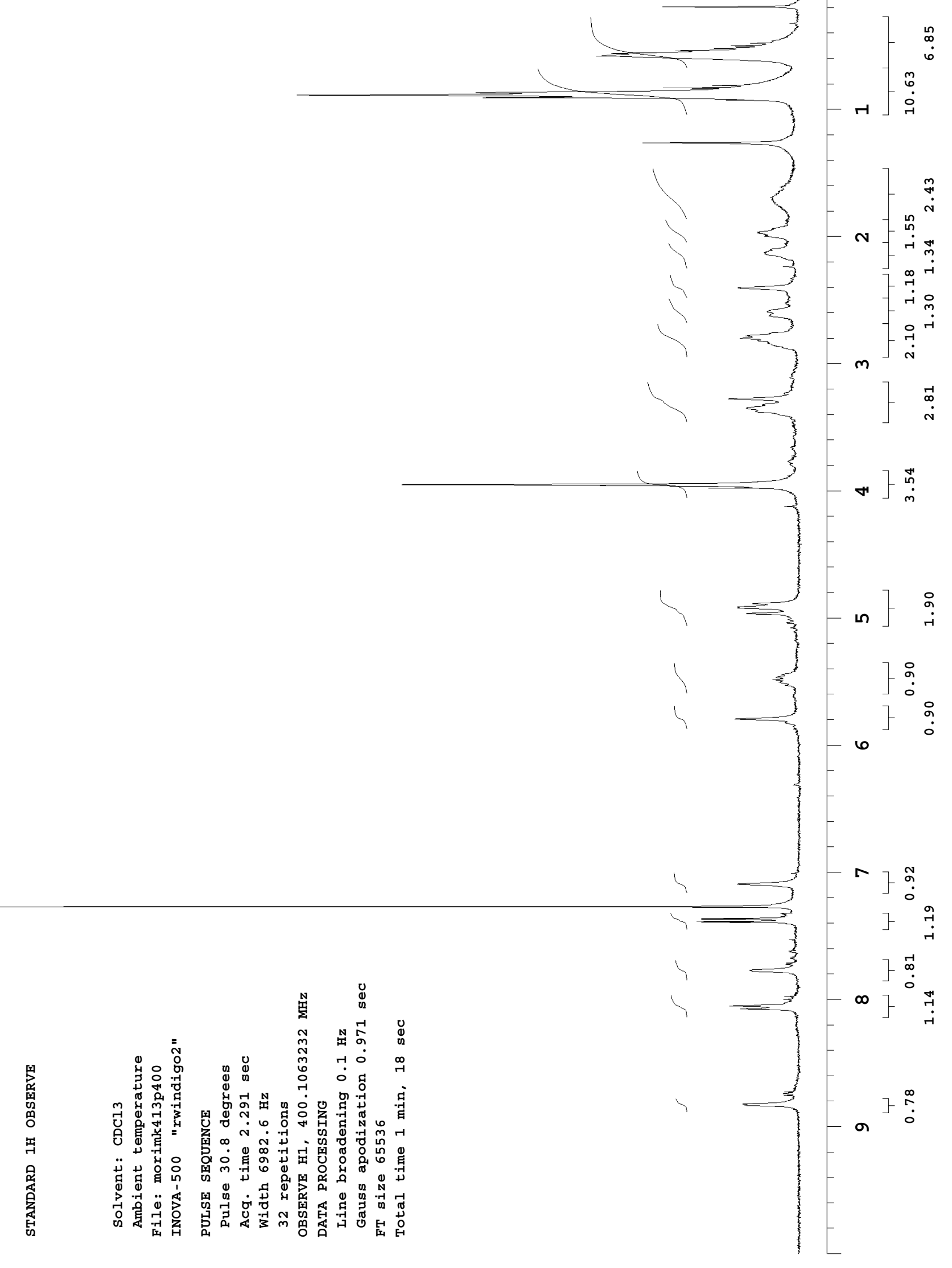




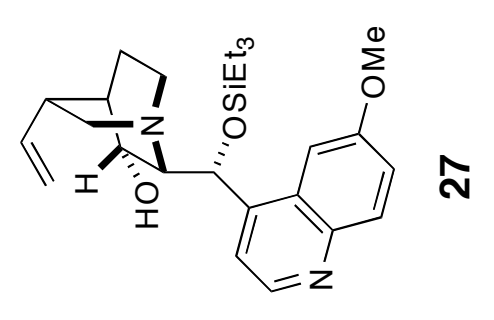

Page S68

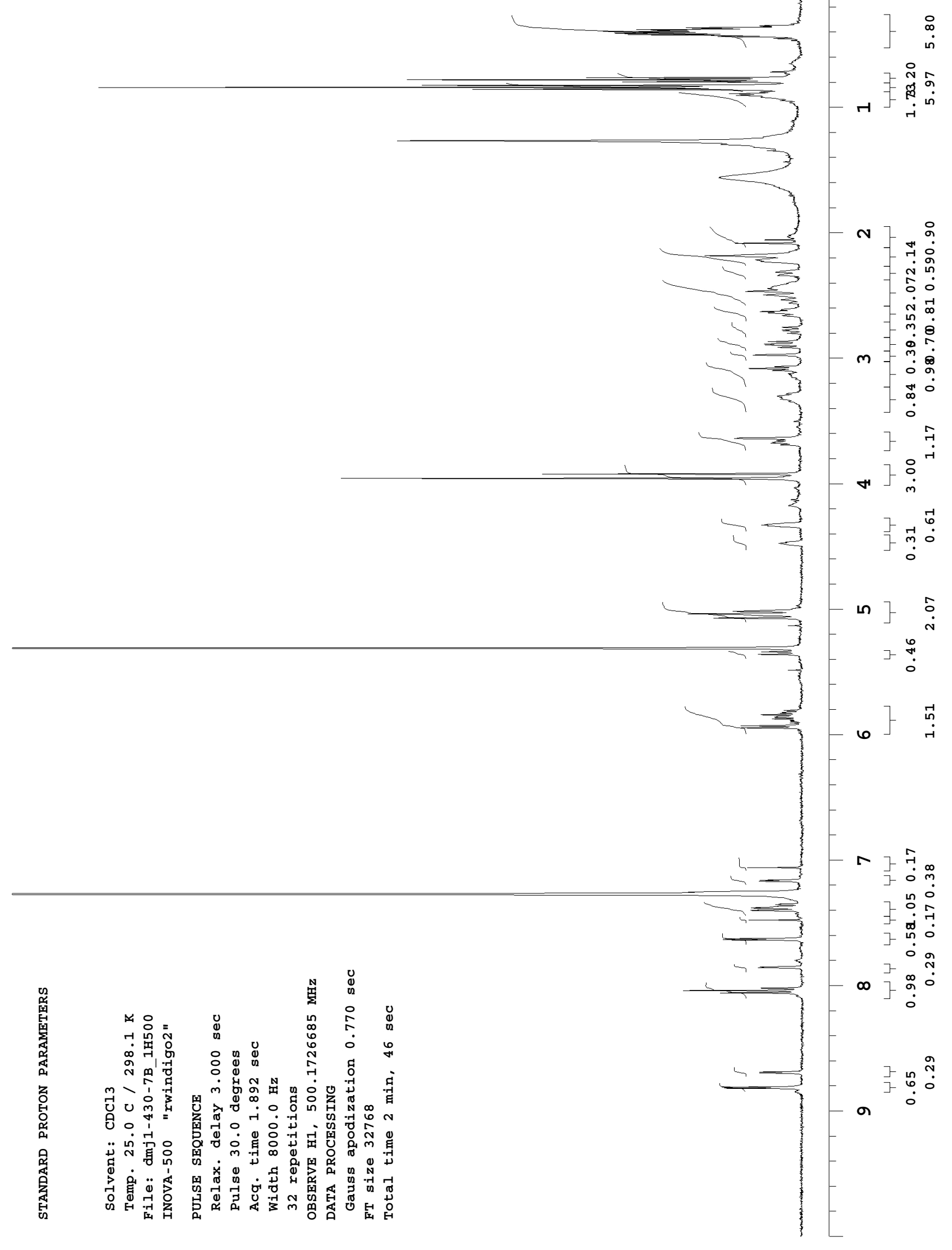


Page S69
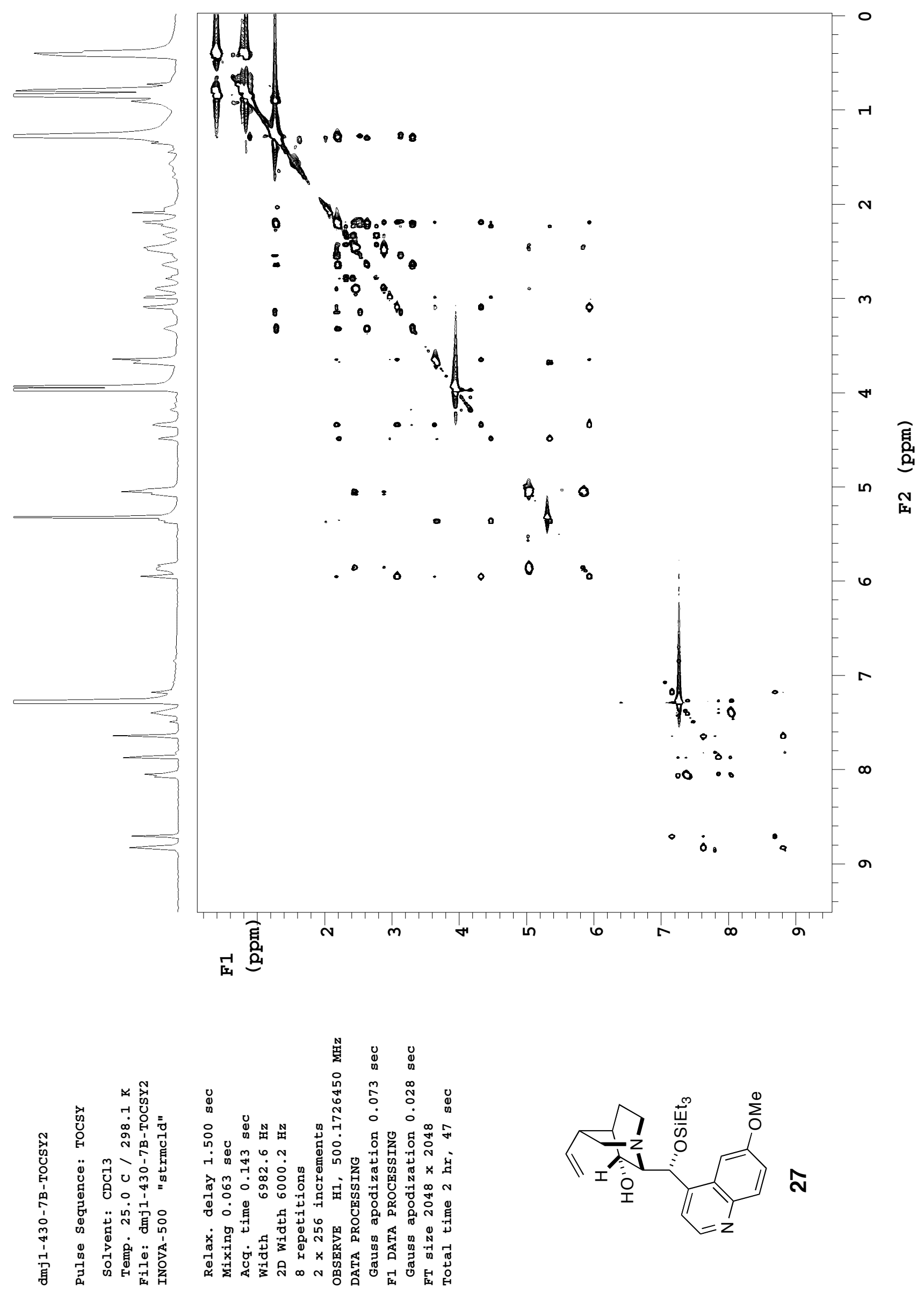
Page S70
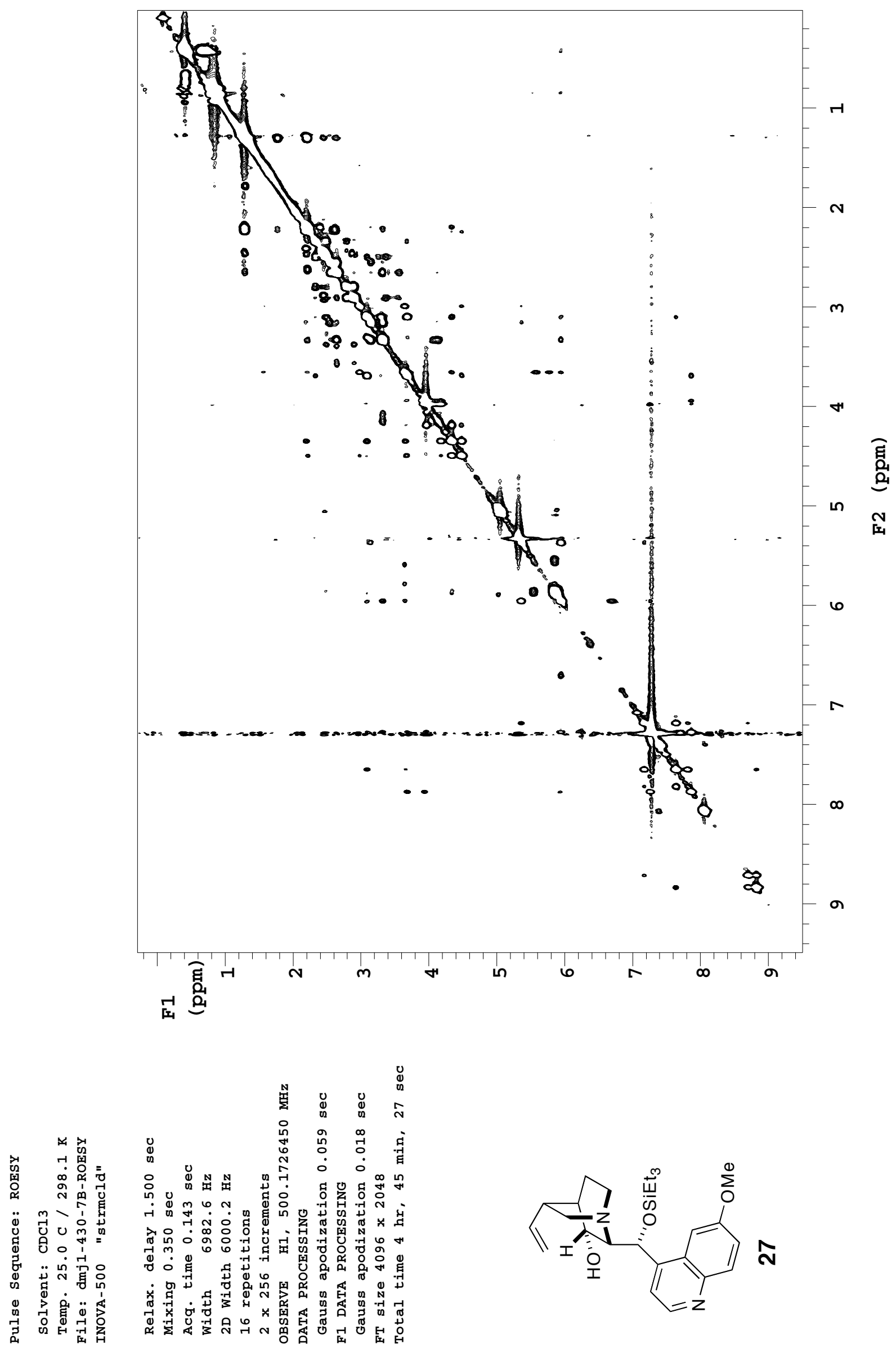
Page S70
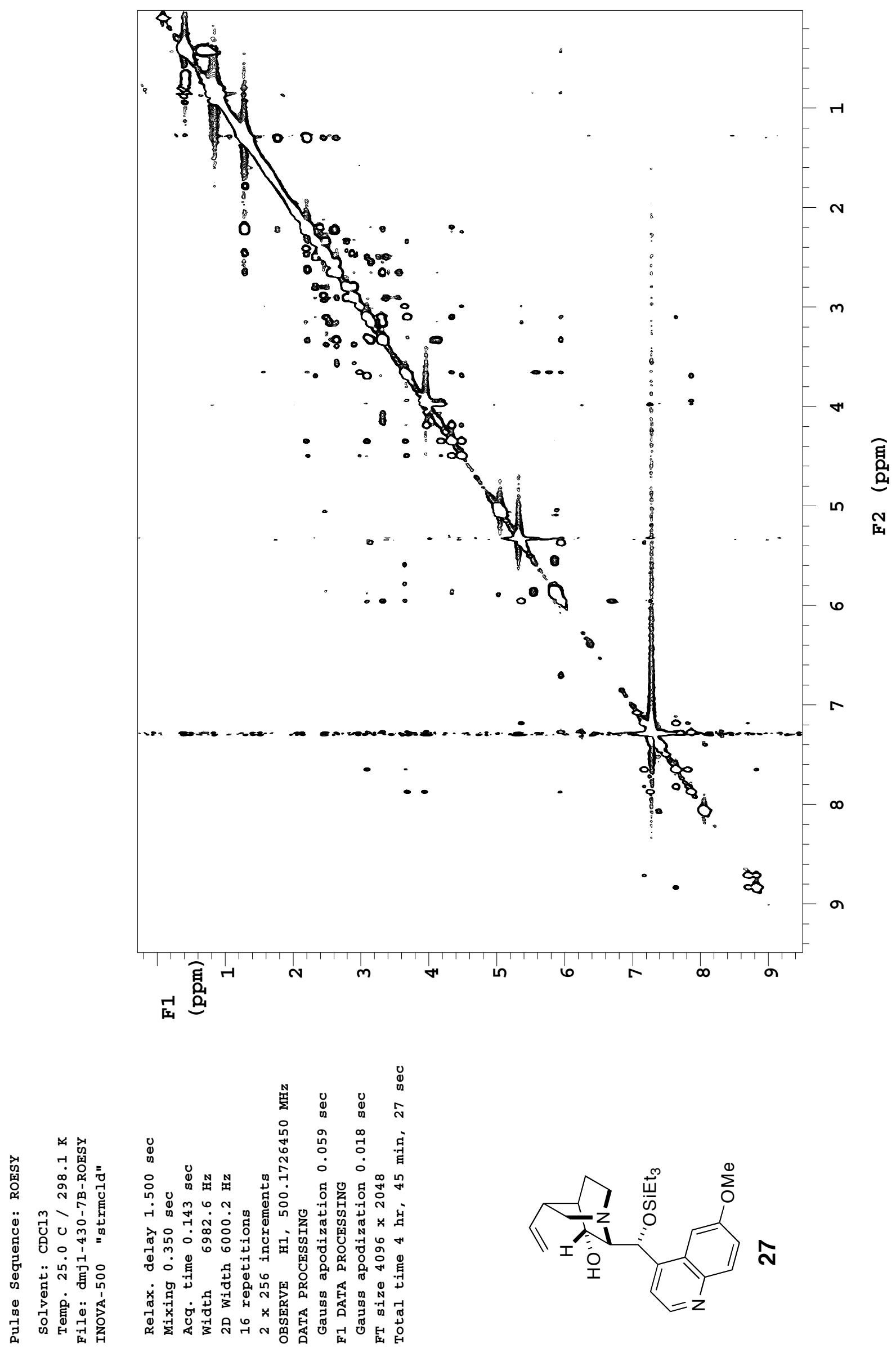

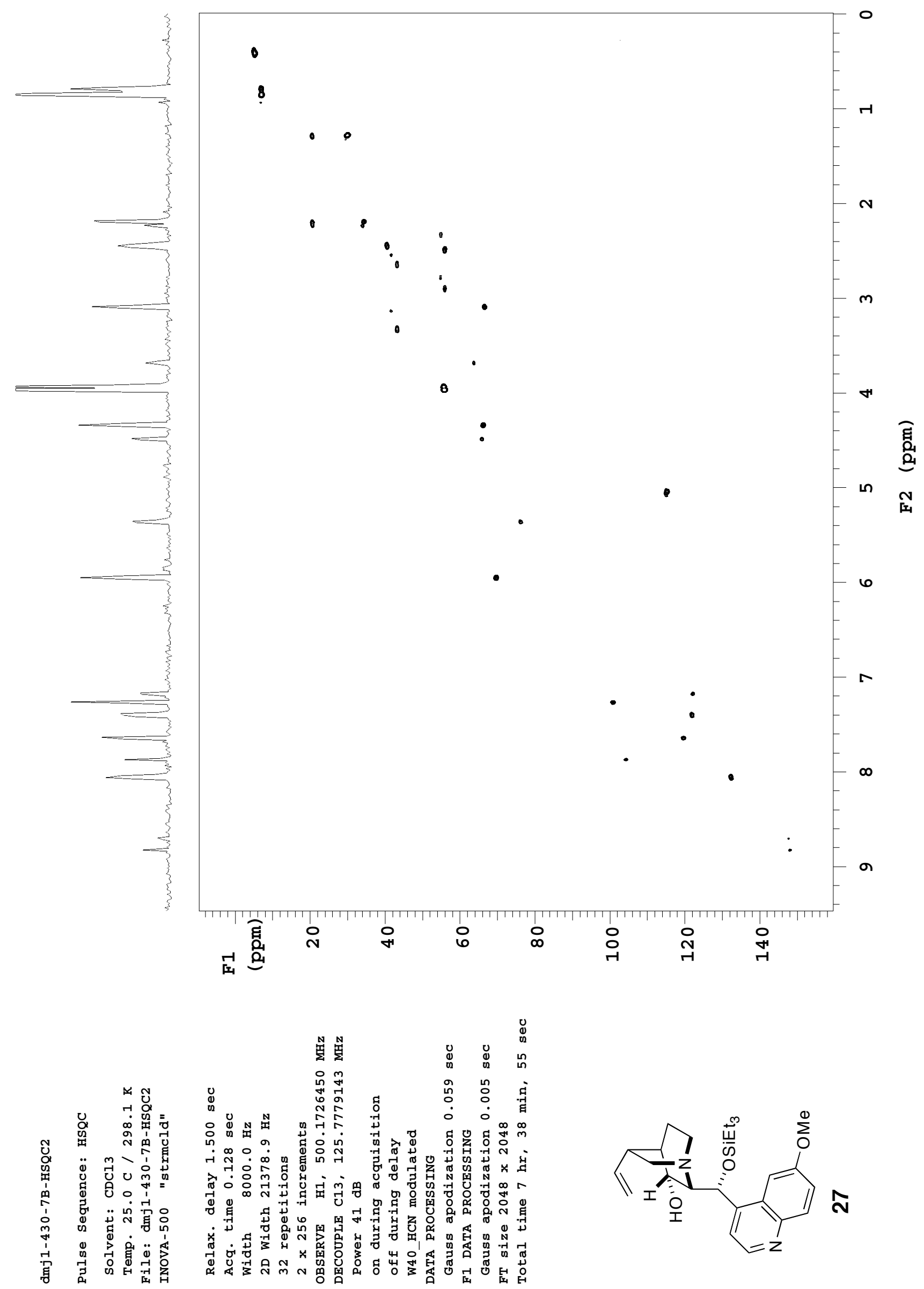

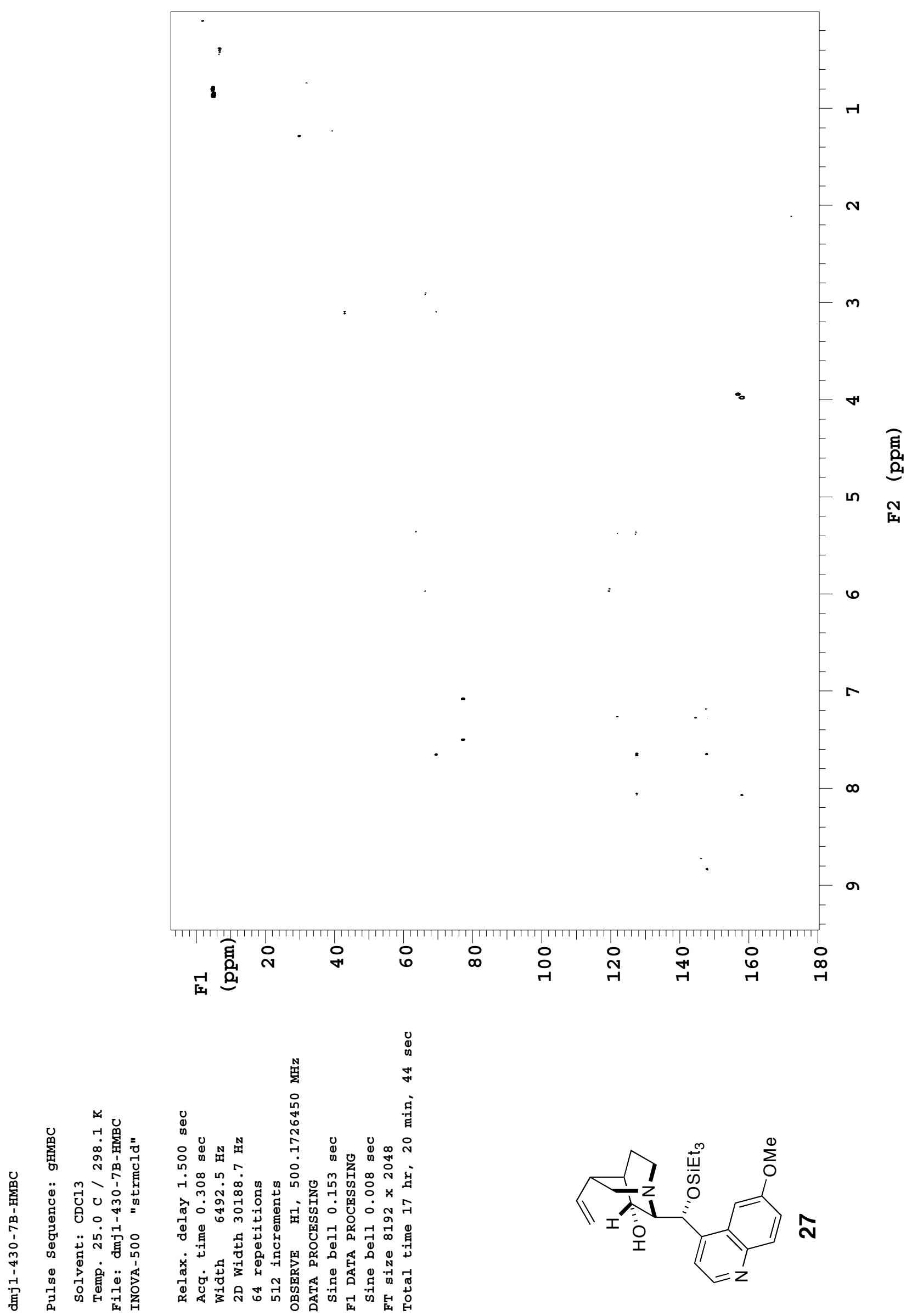
Page S73
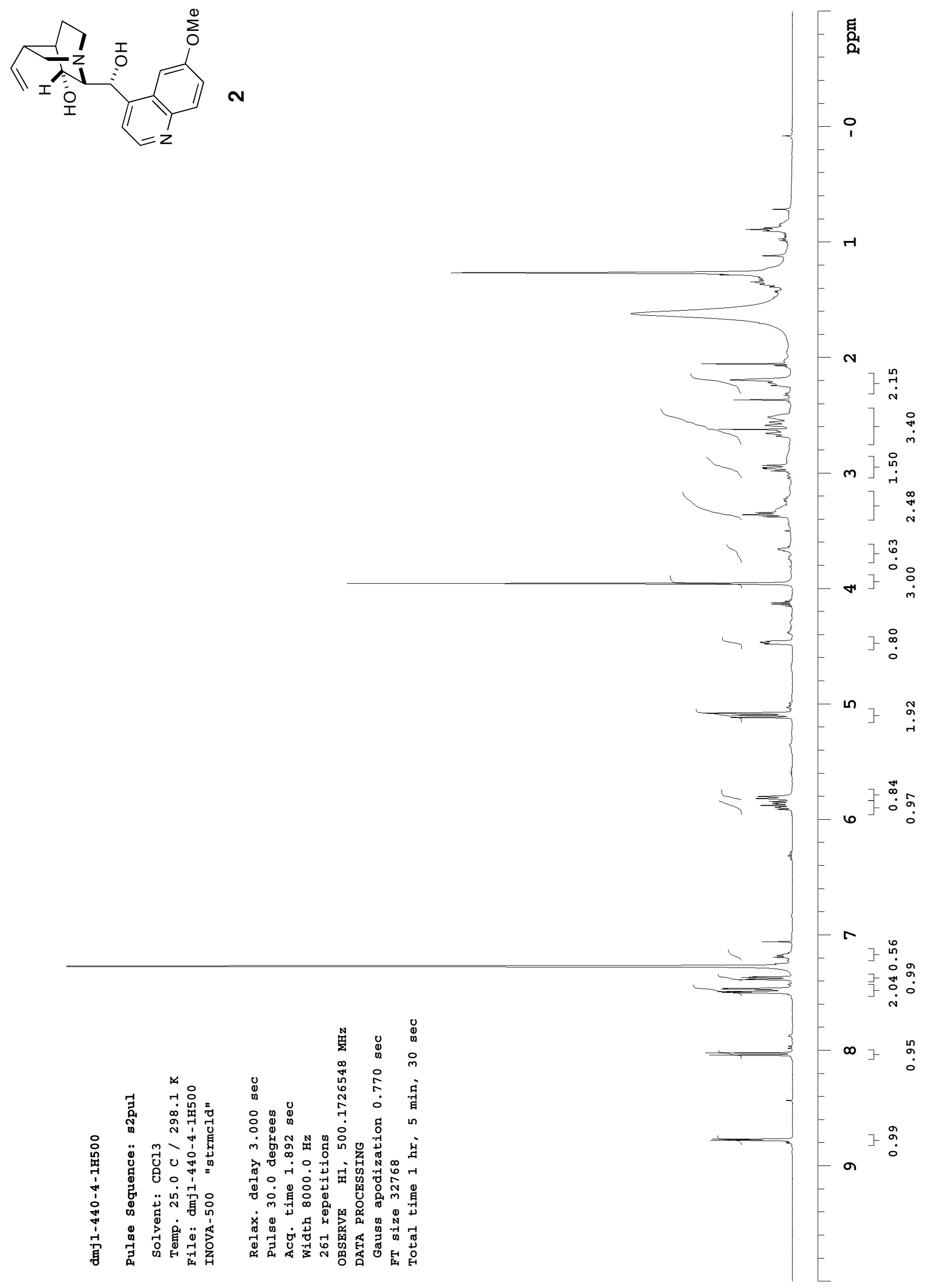
Page S74
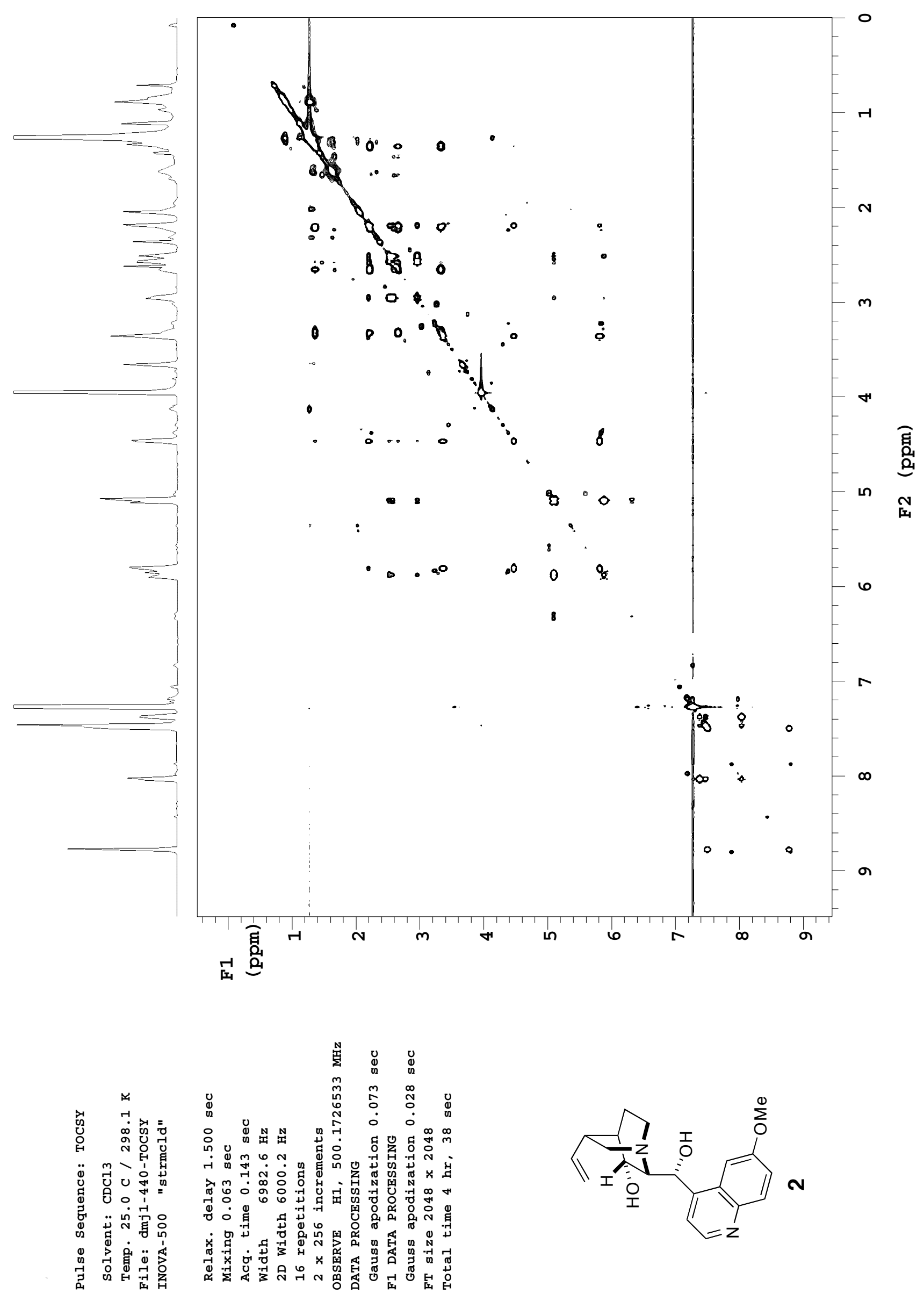

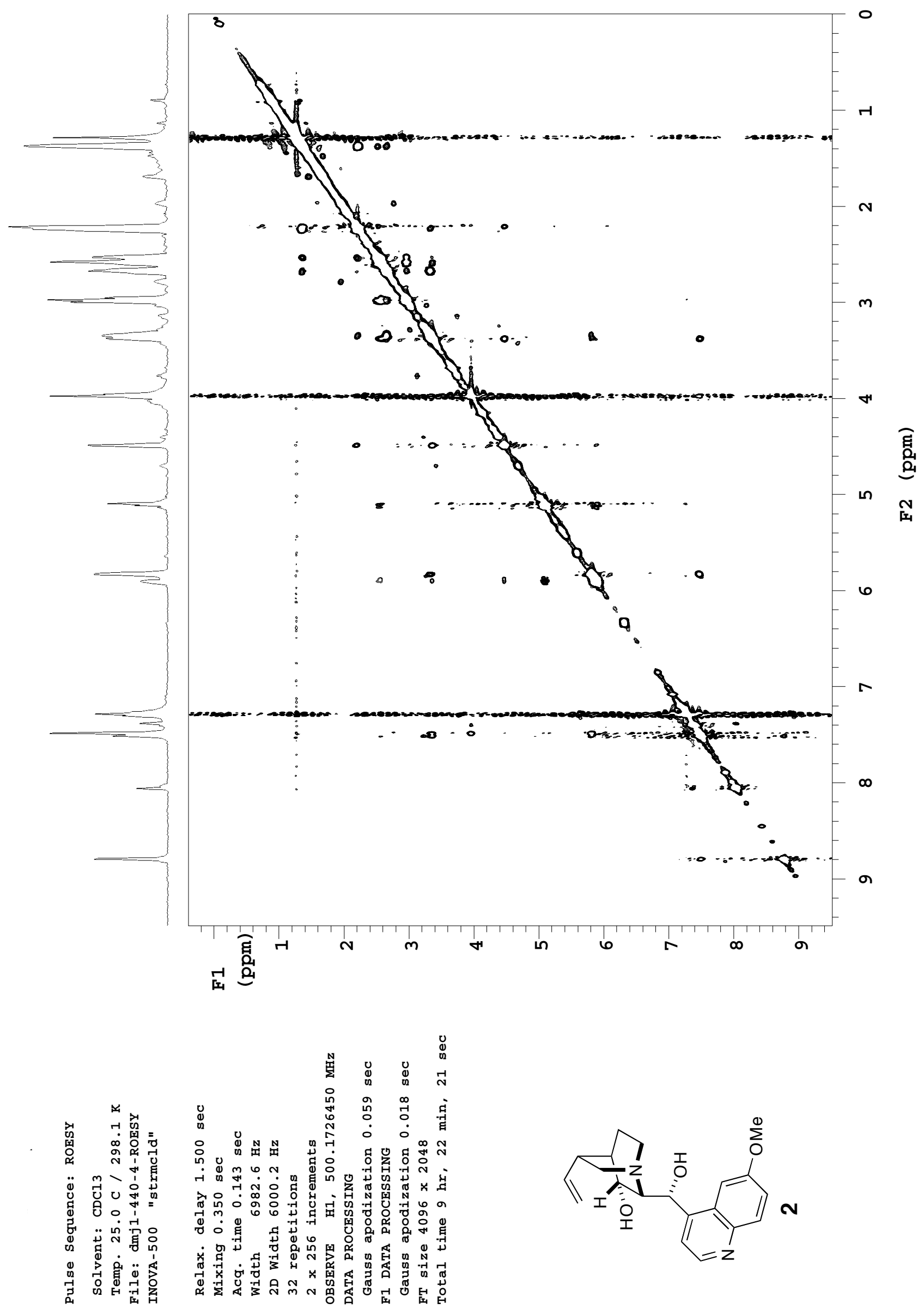
Page S76
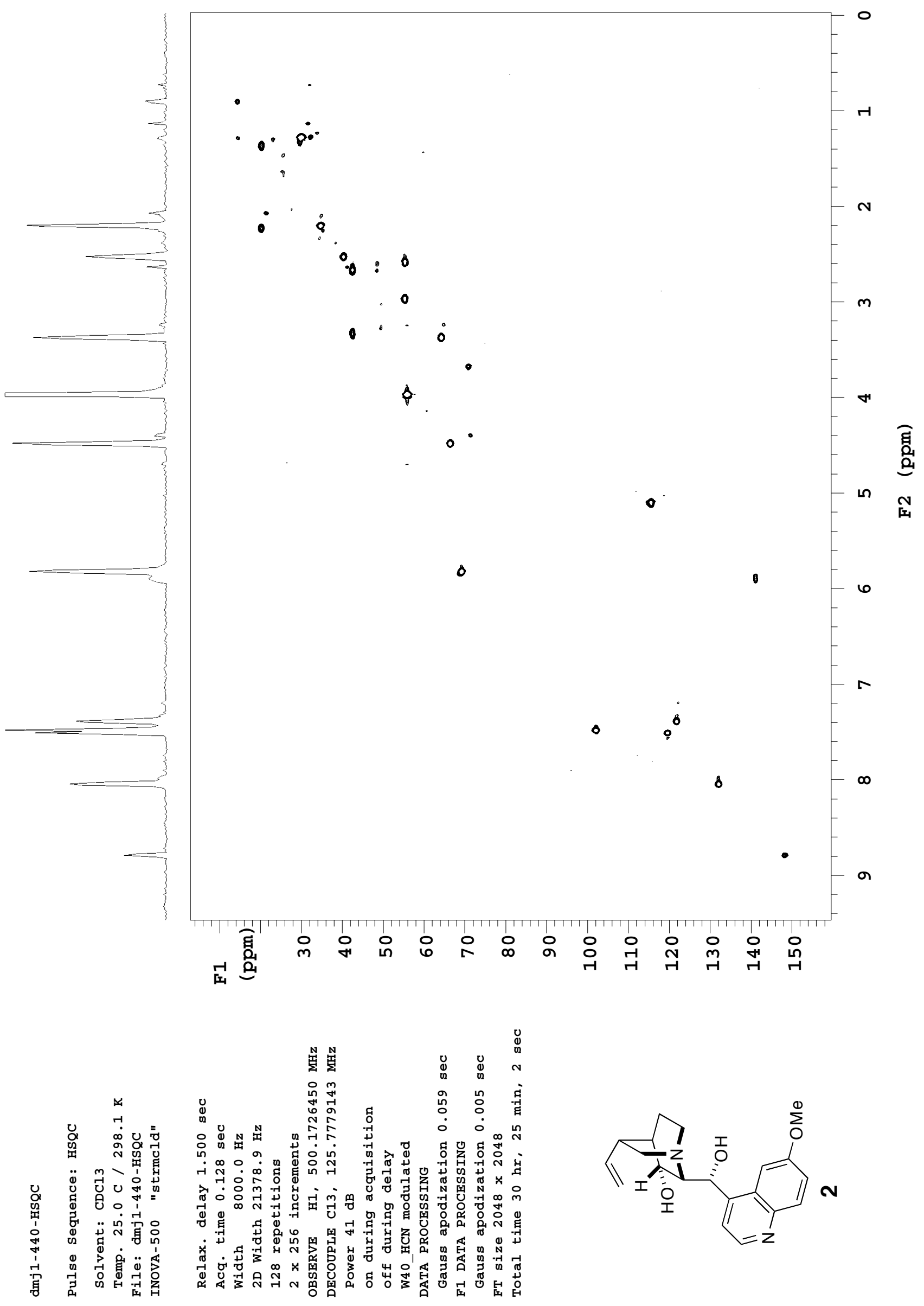
Page S77
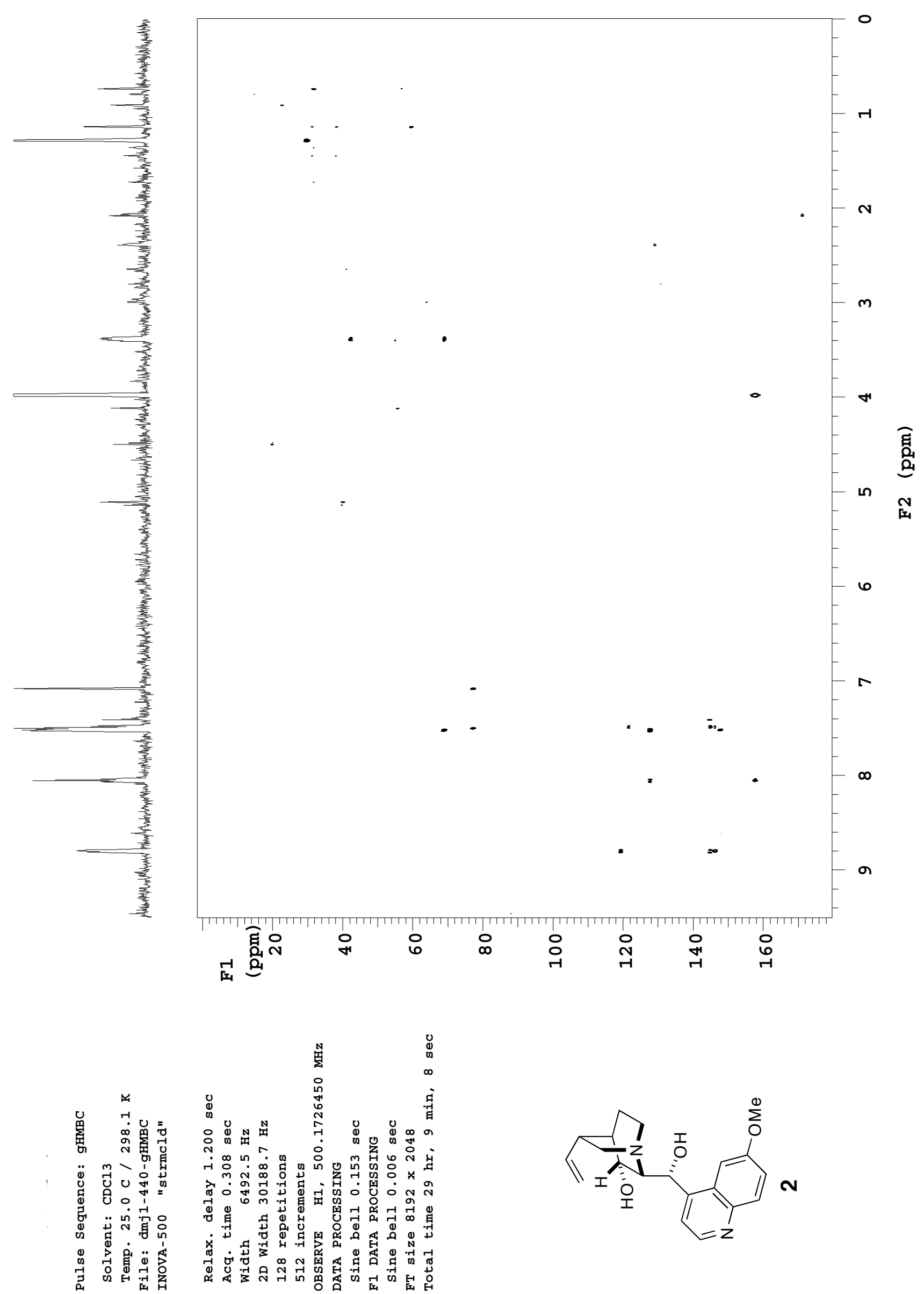
Page S78

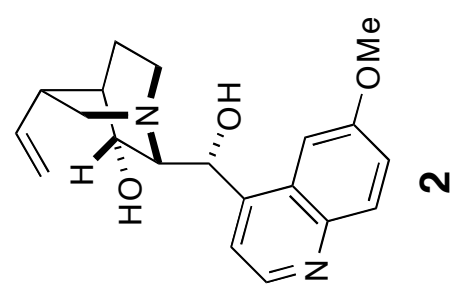
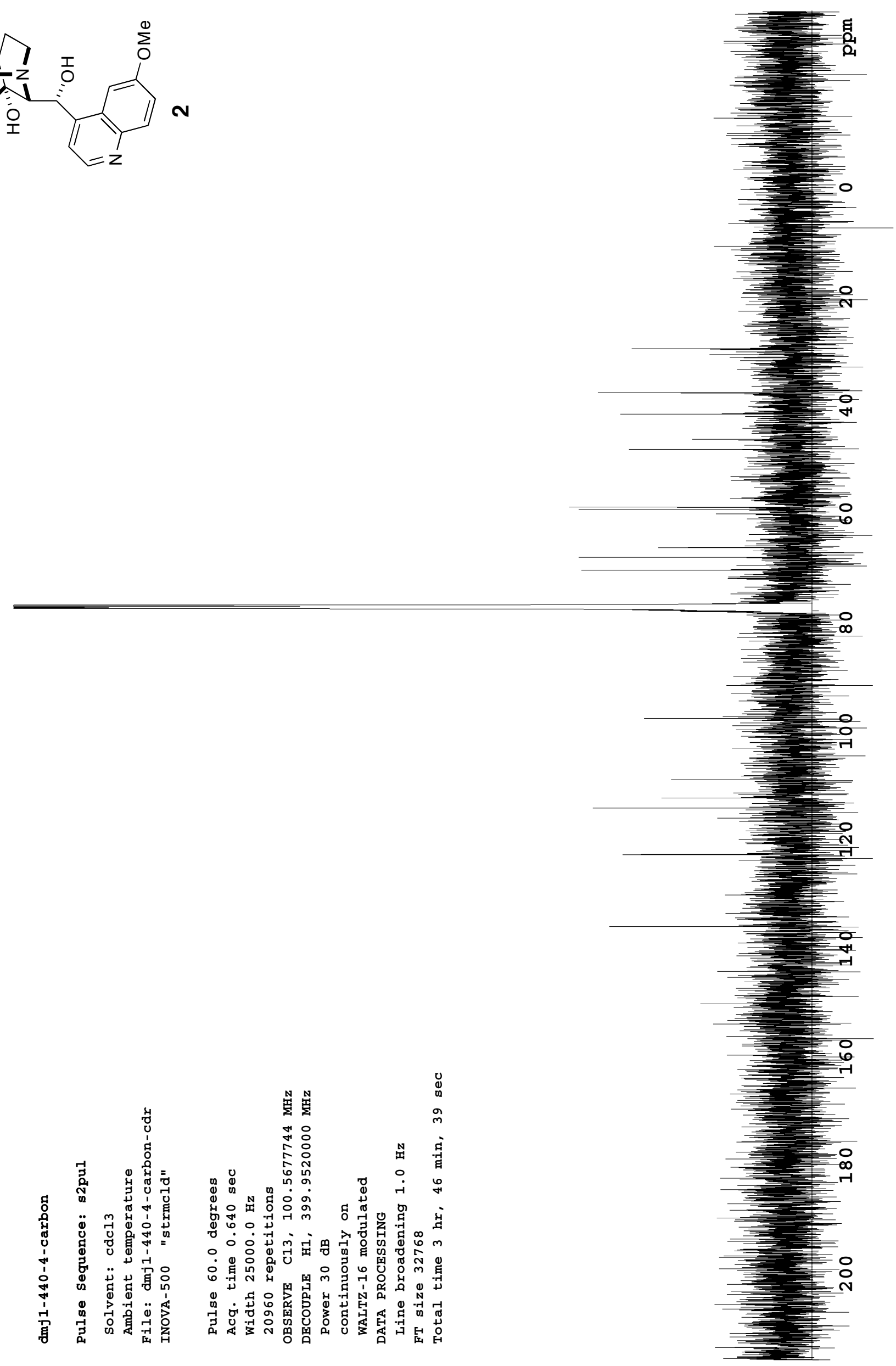
Page S79

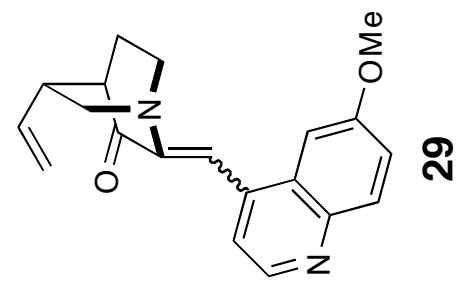

몀
م)

0

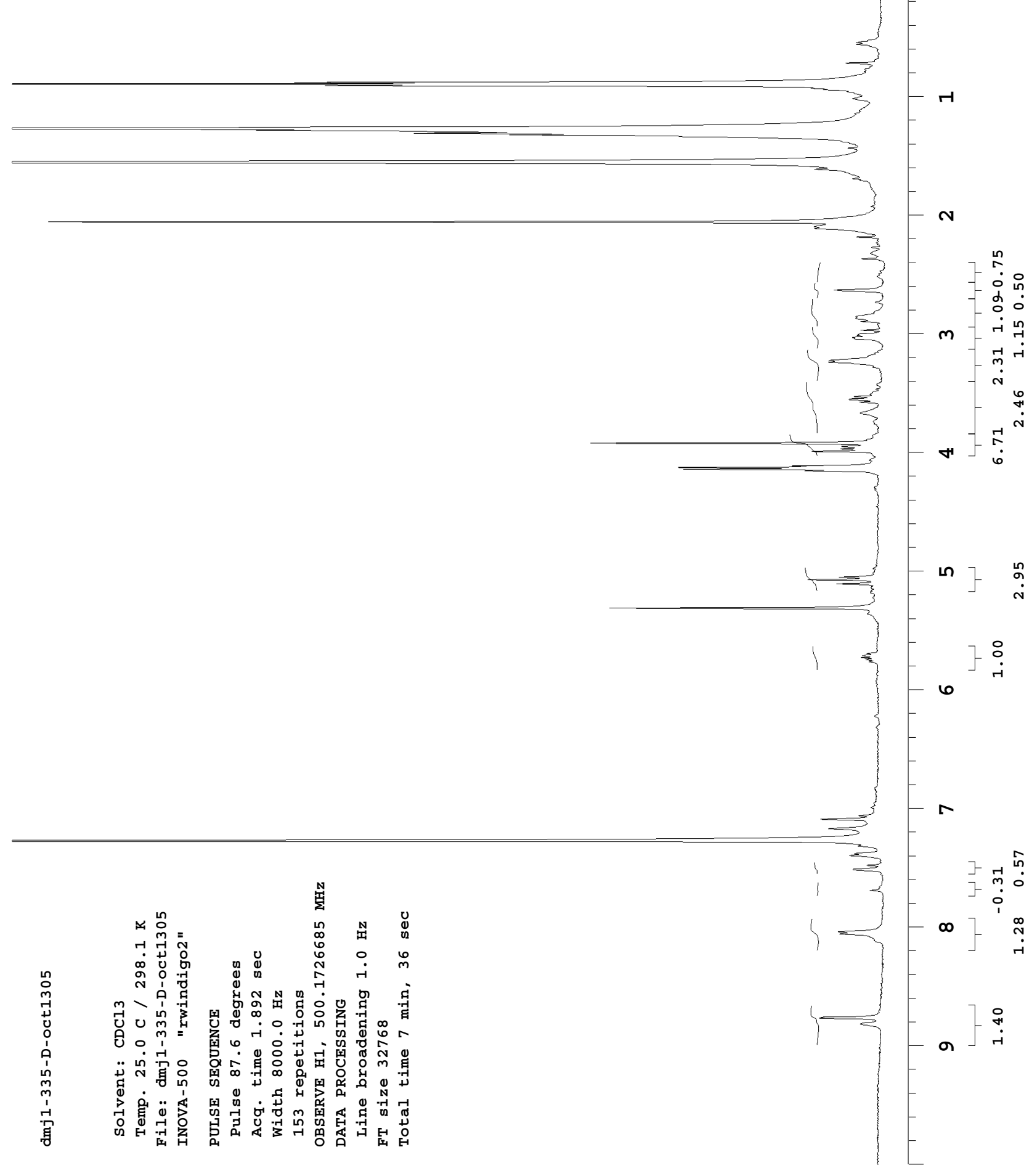


Page S80
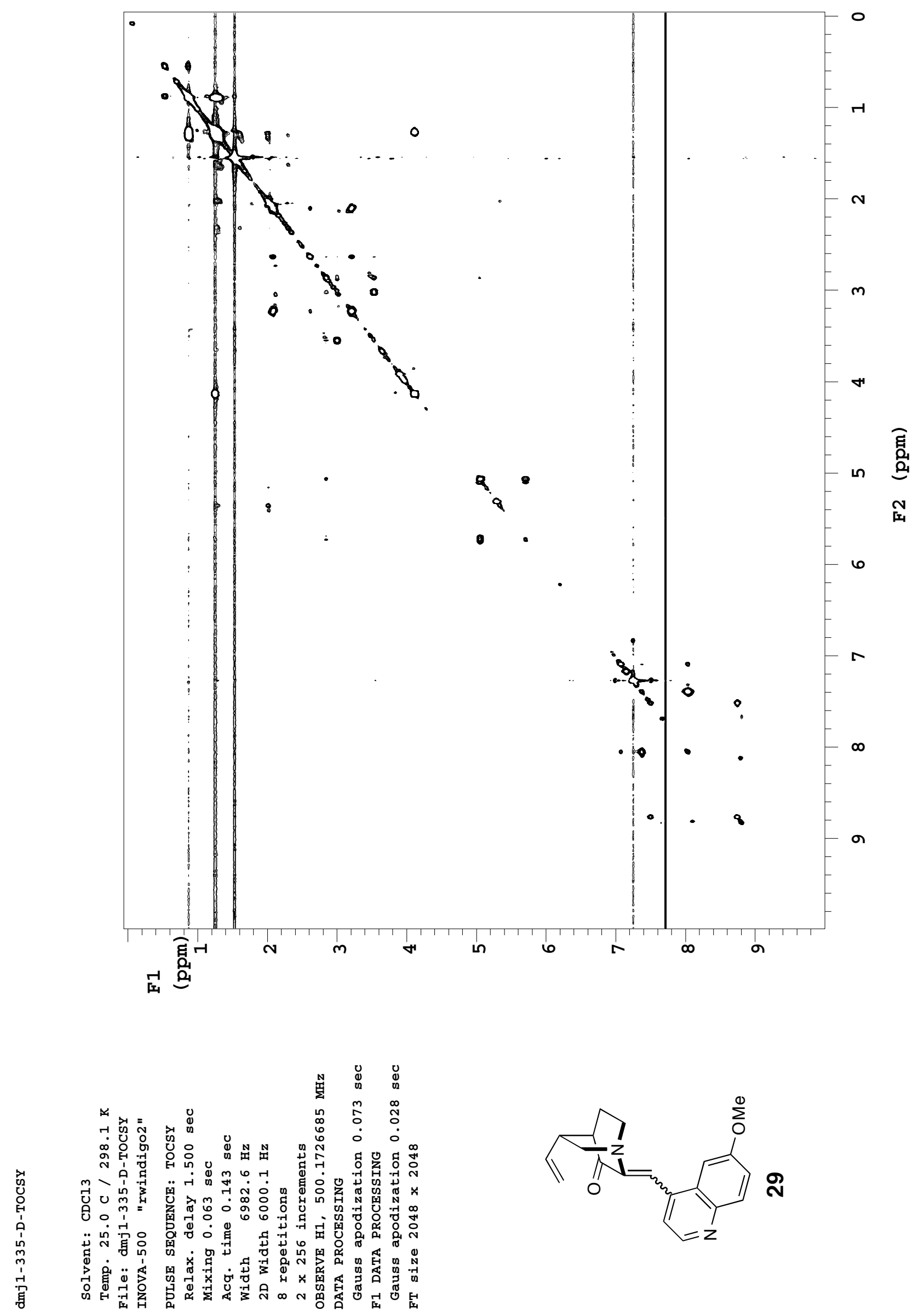
Page S81
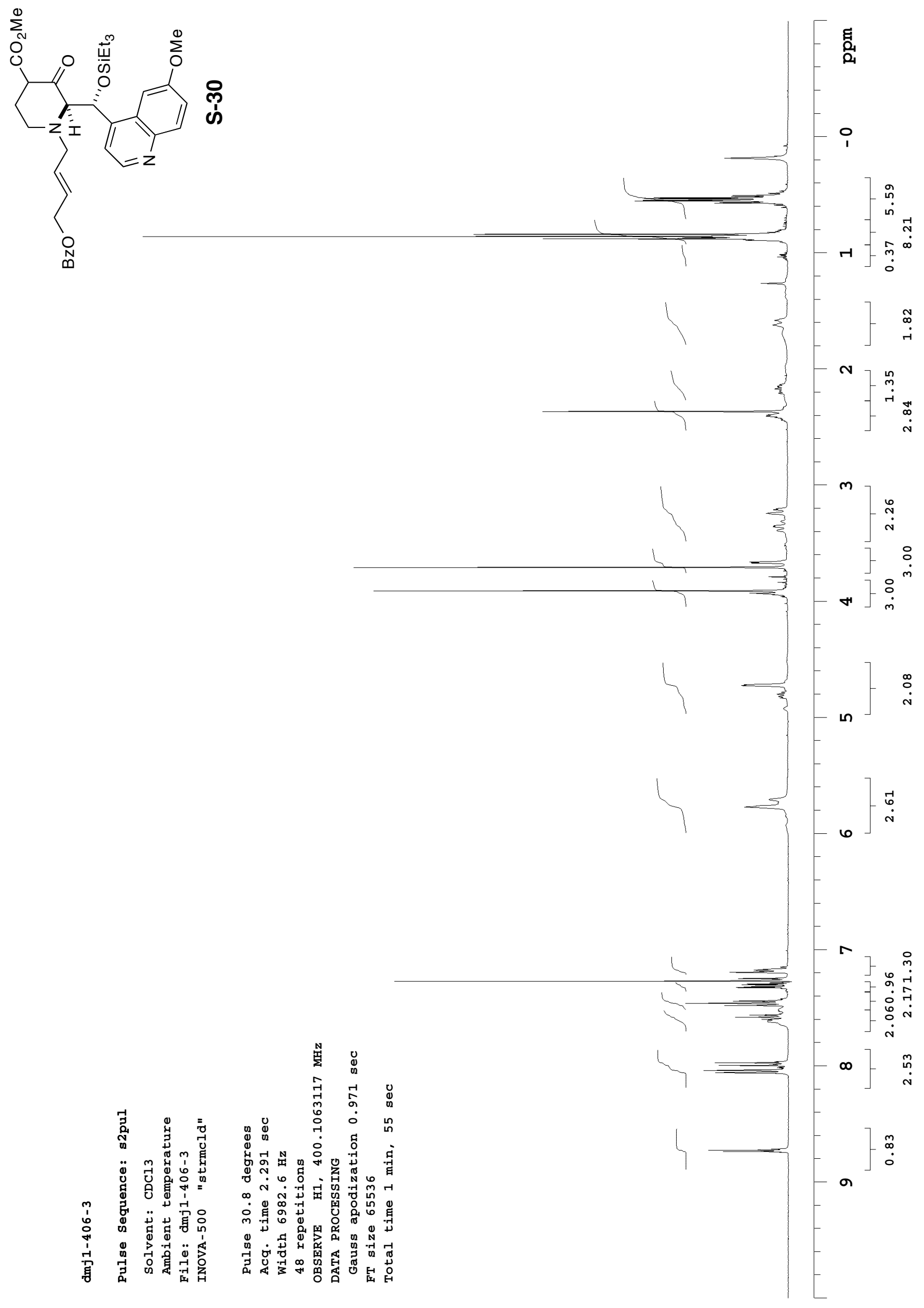


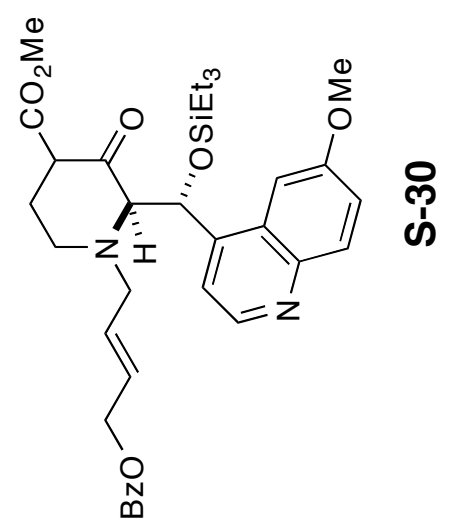

Page S82

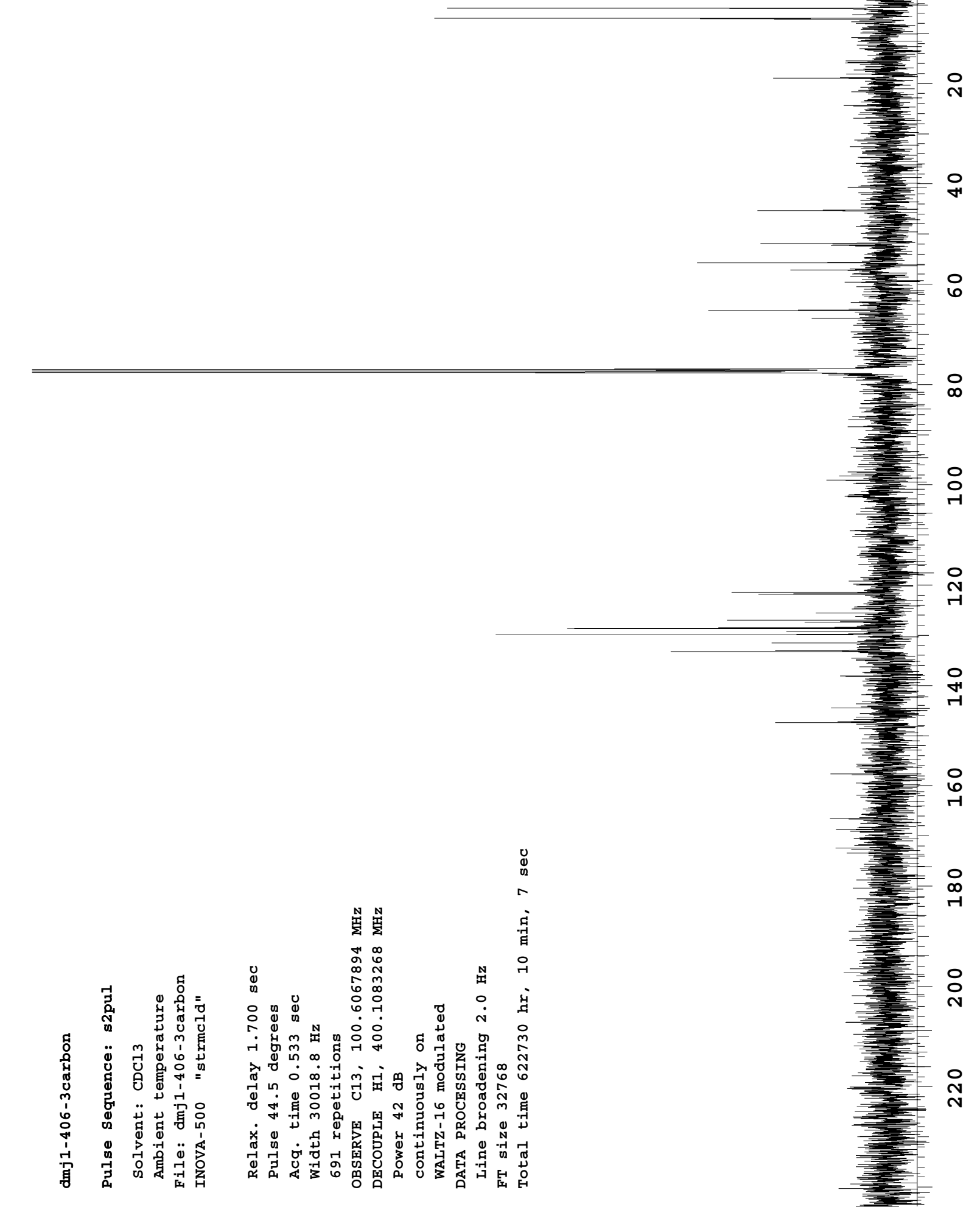


Page S83
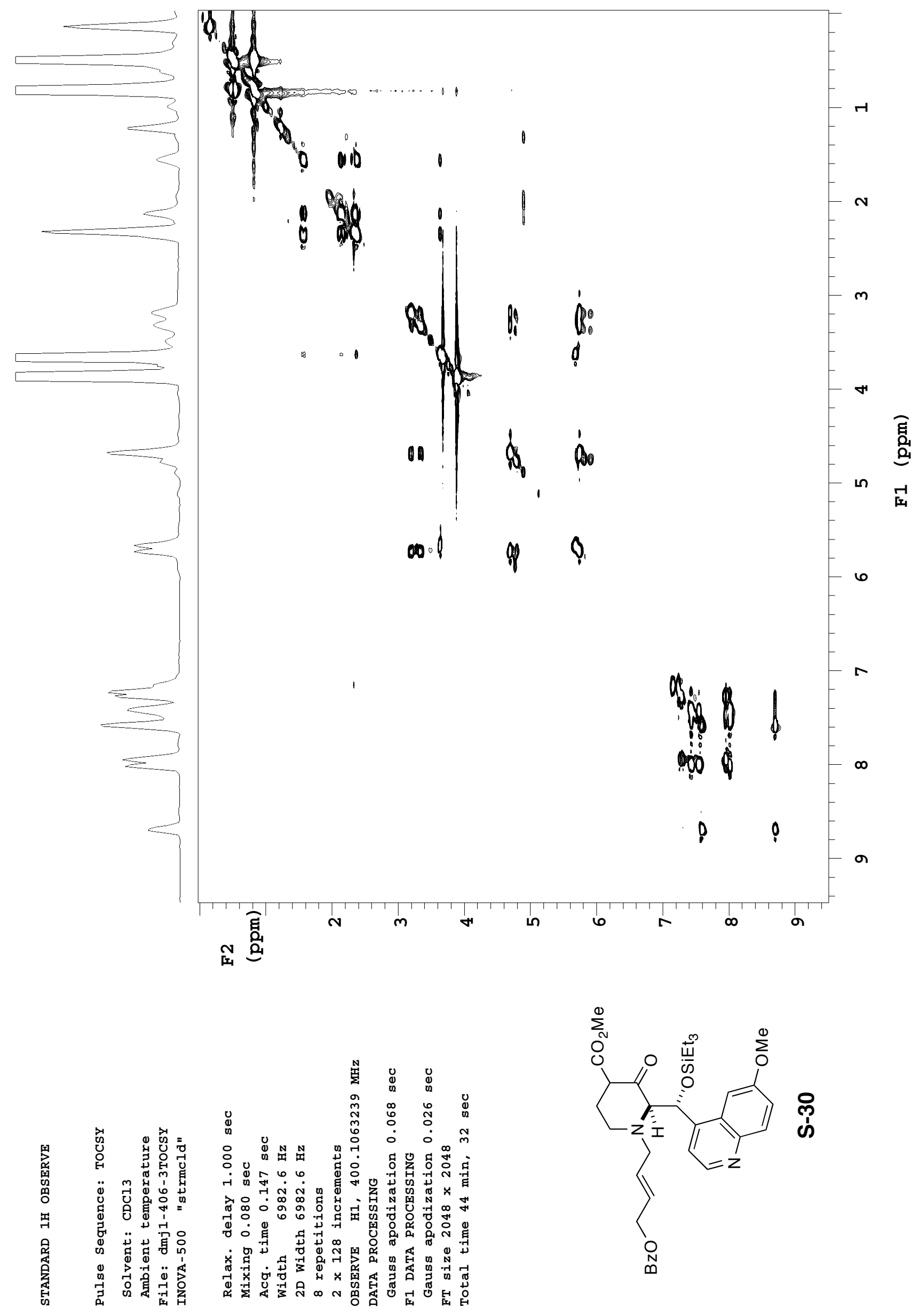


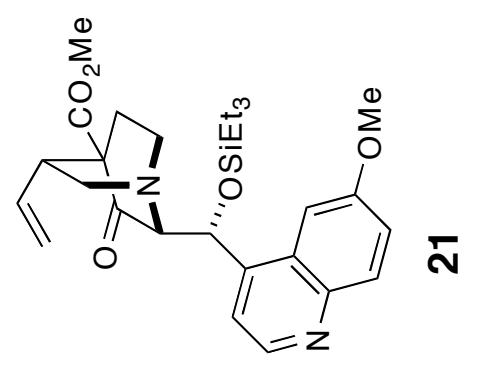

Page S84

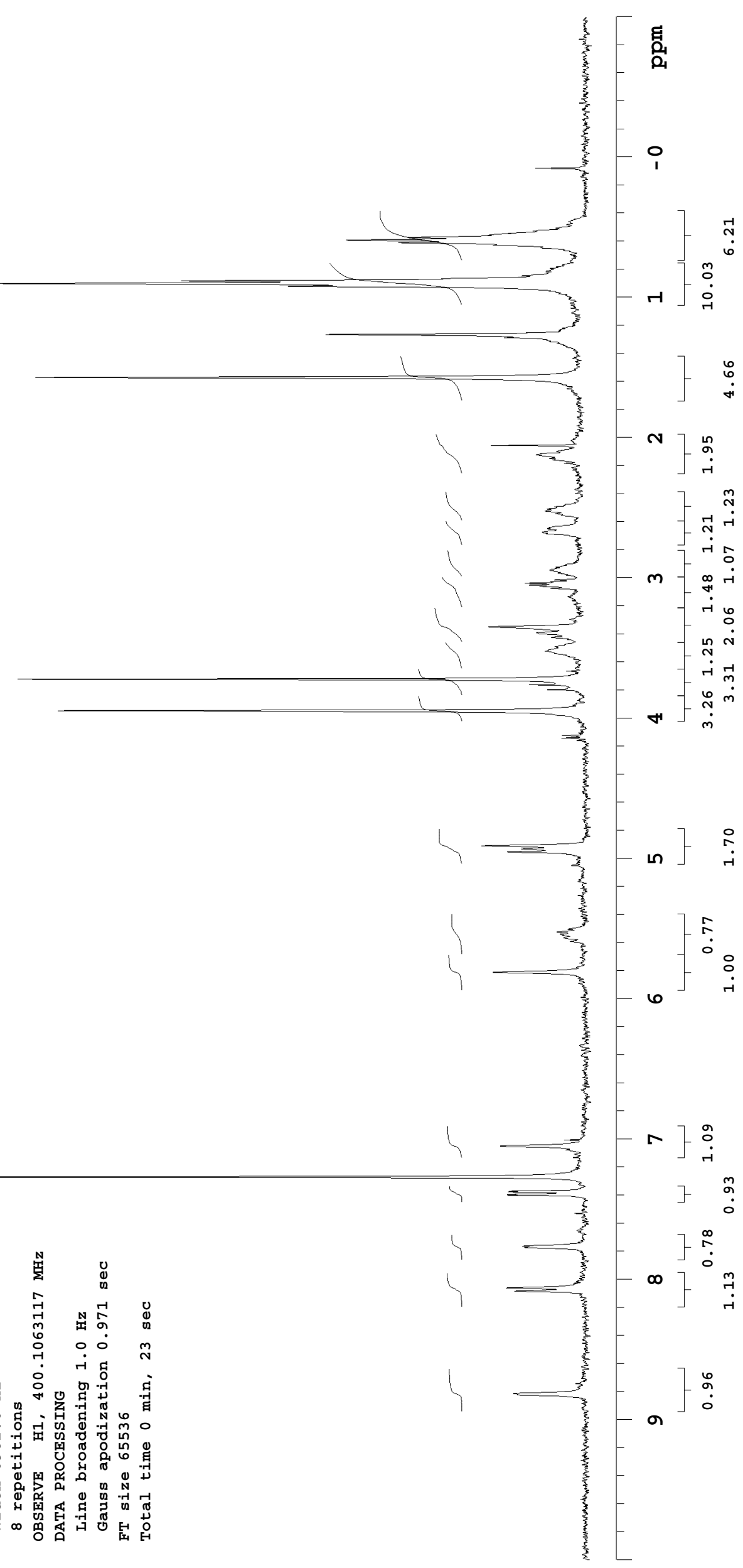


Page S85
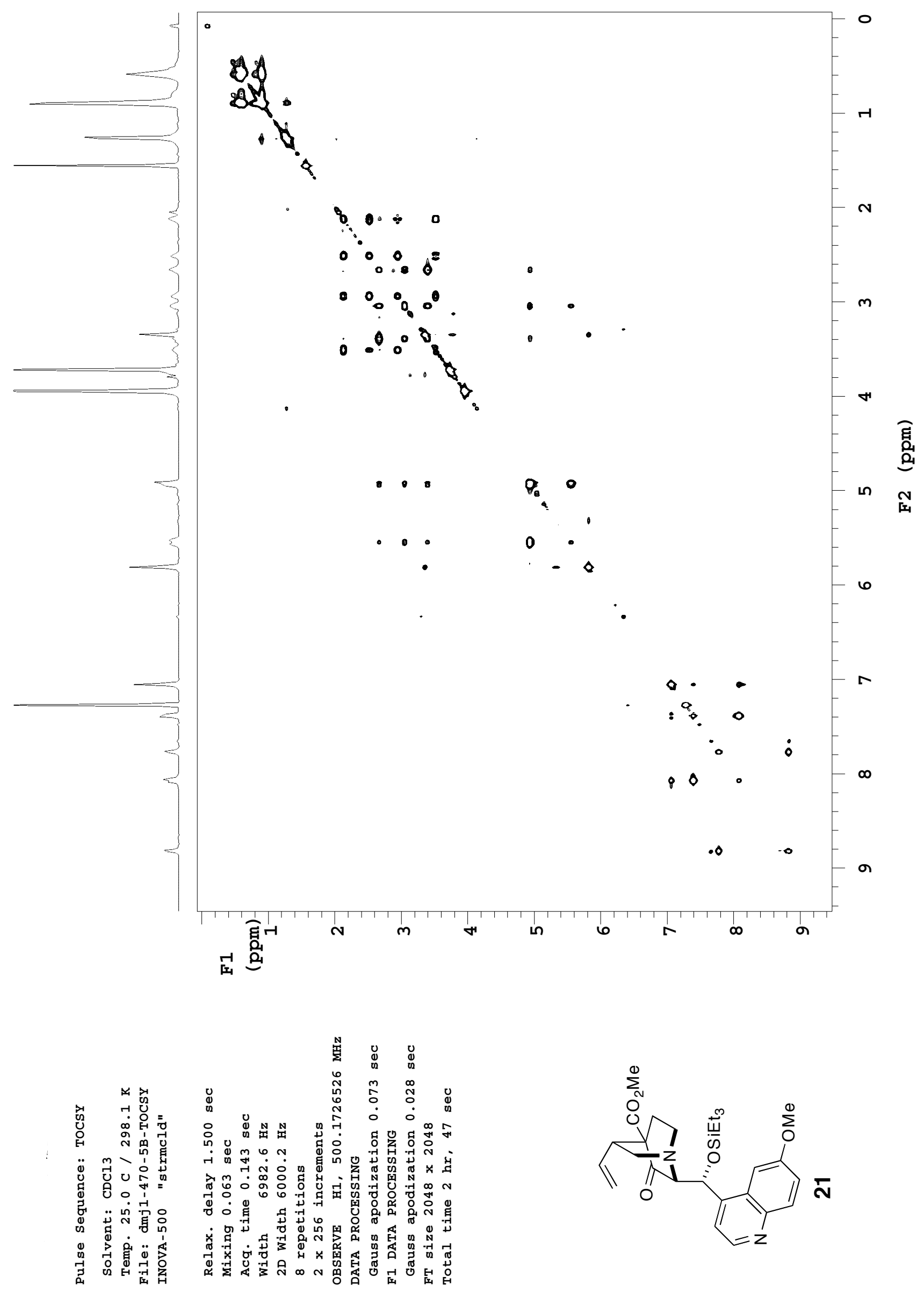

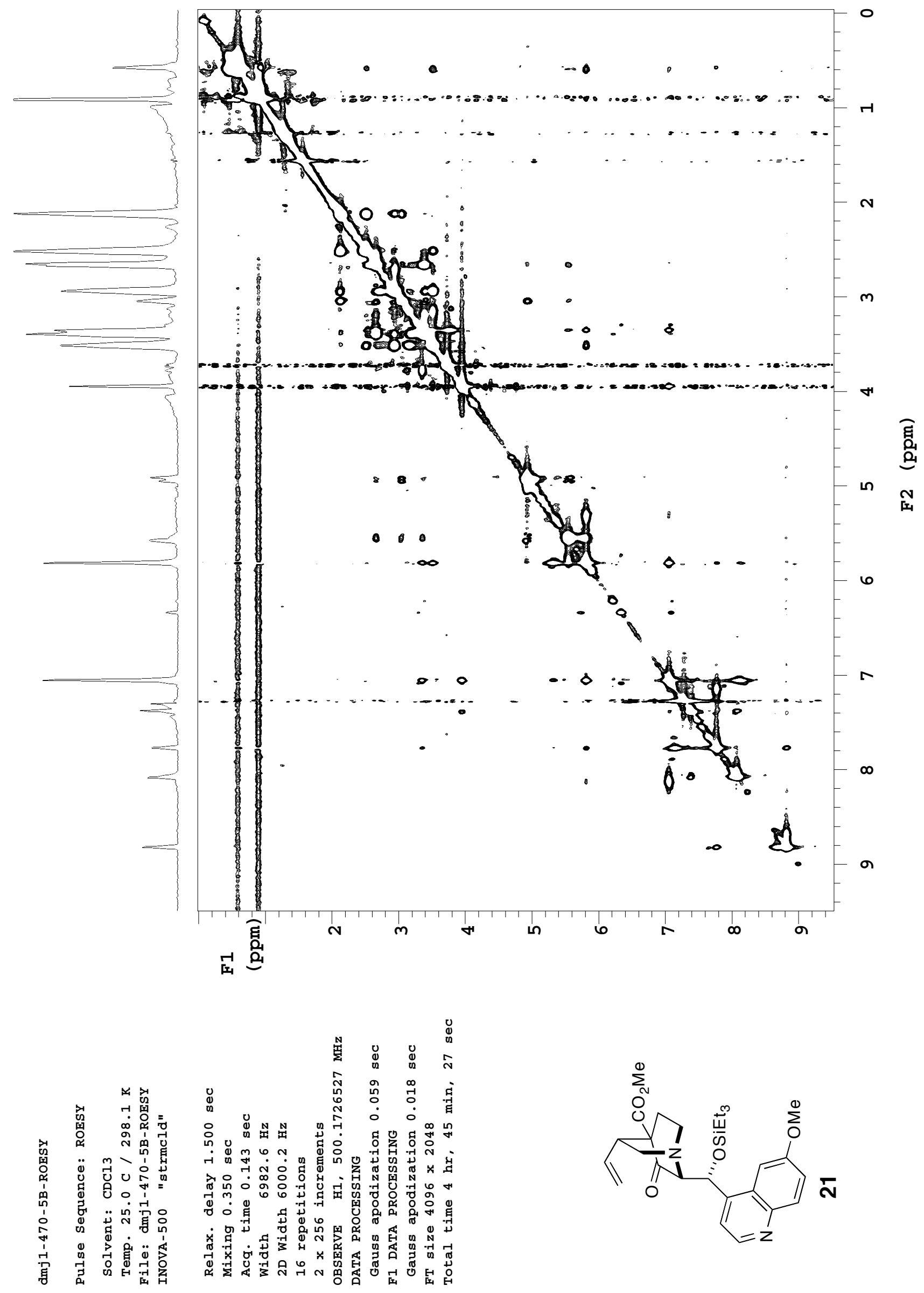
Page S87
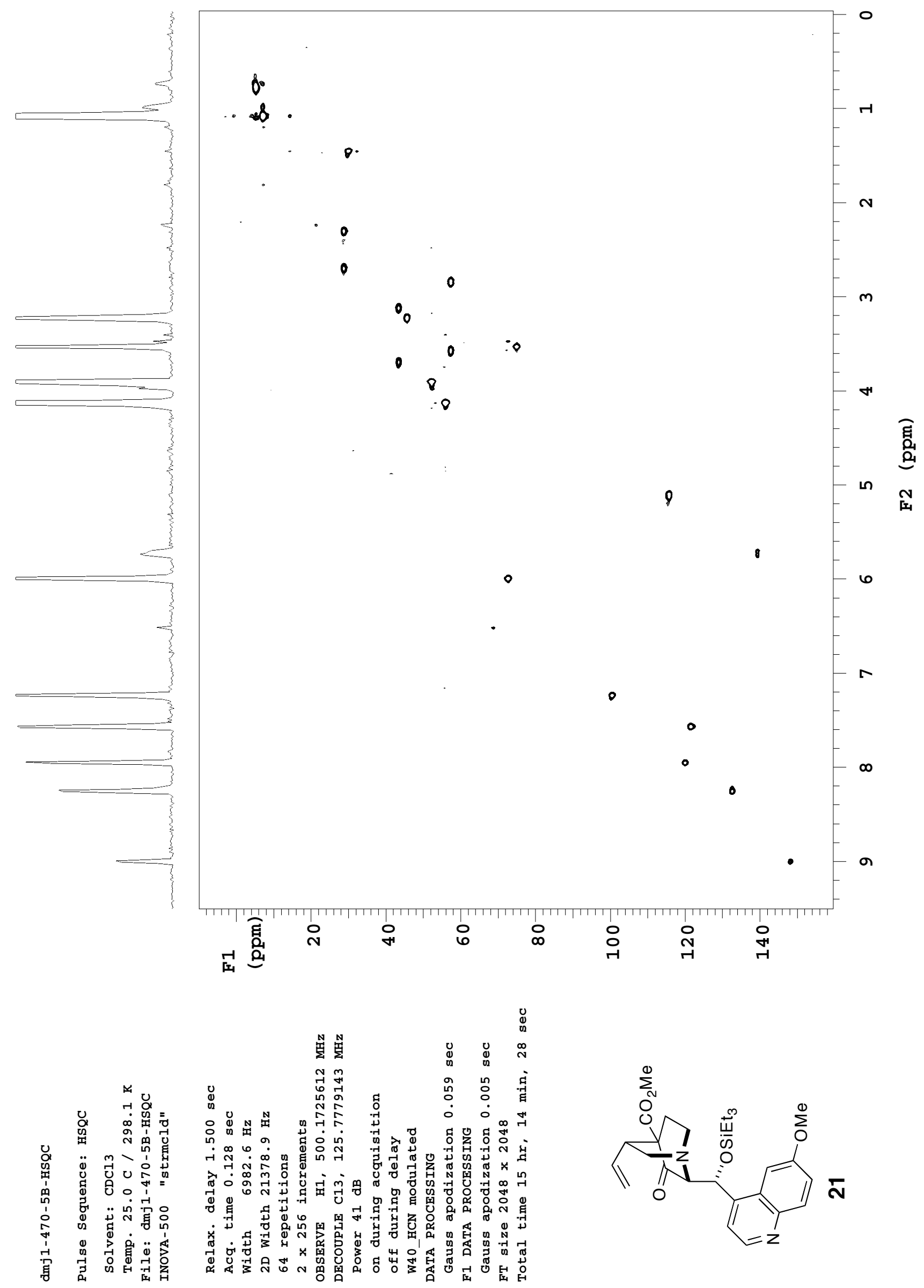


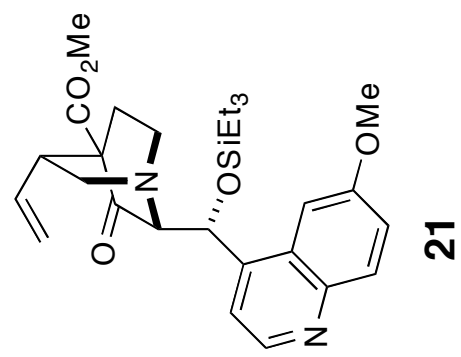

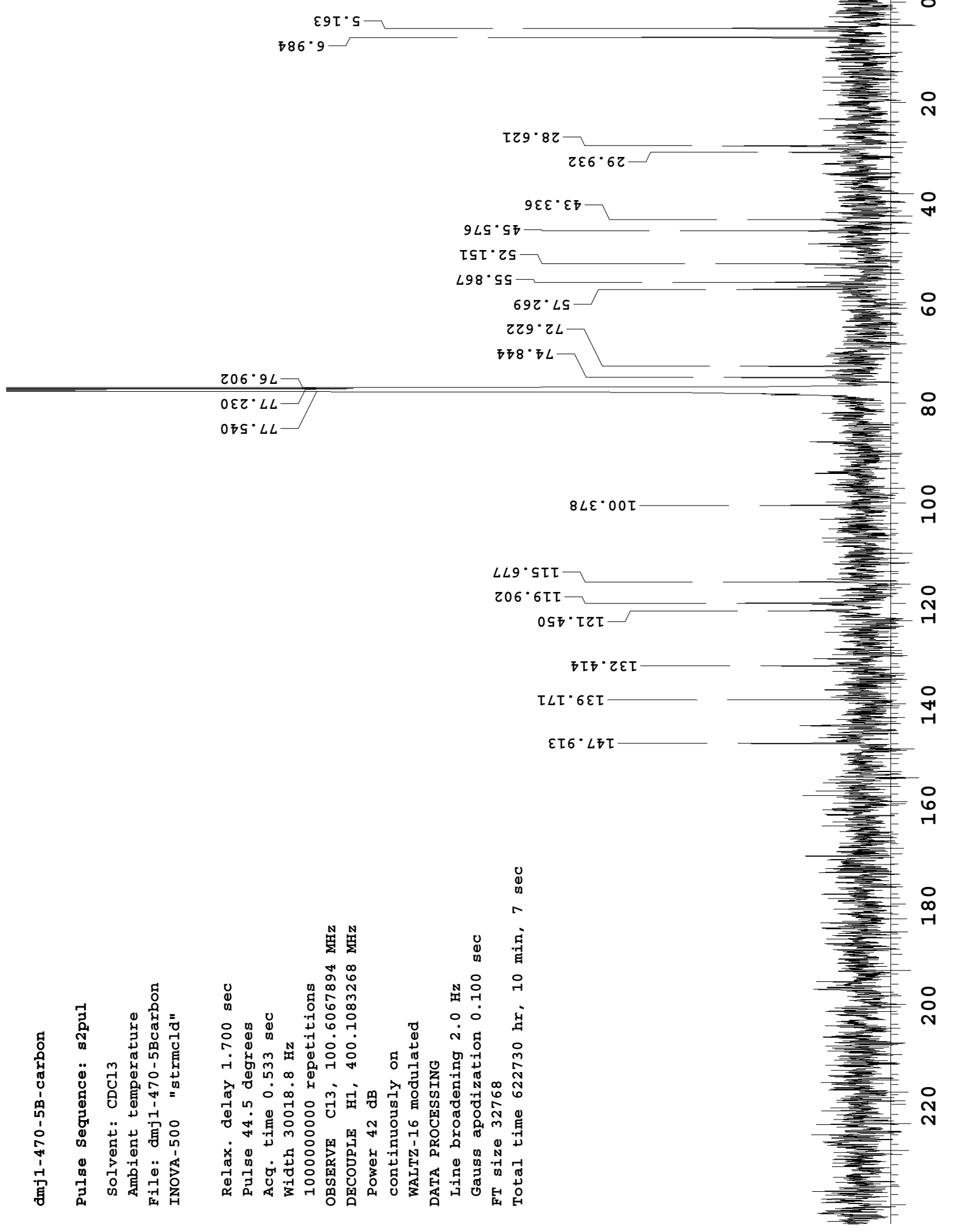




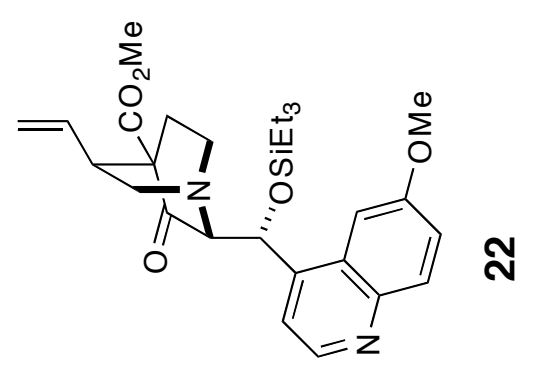

Page S89

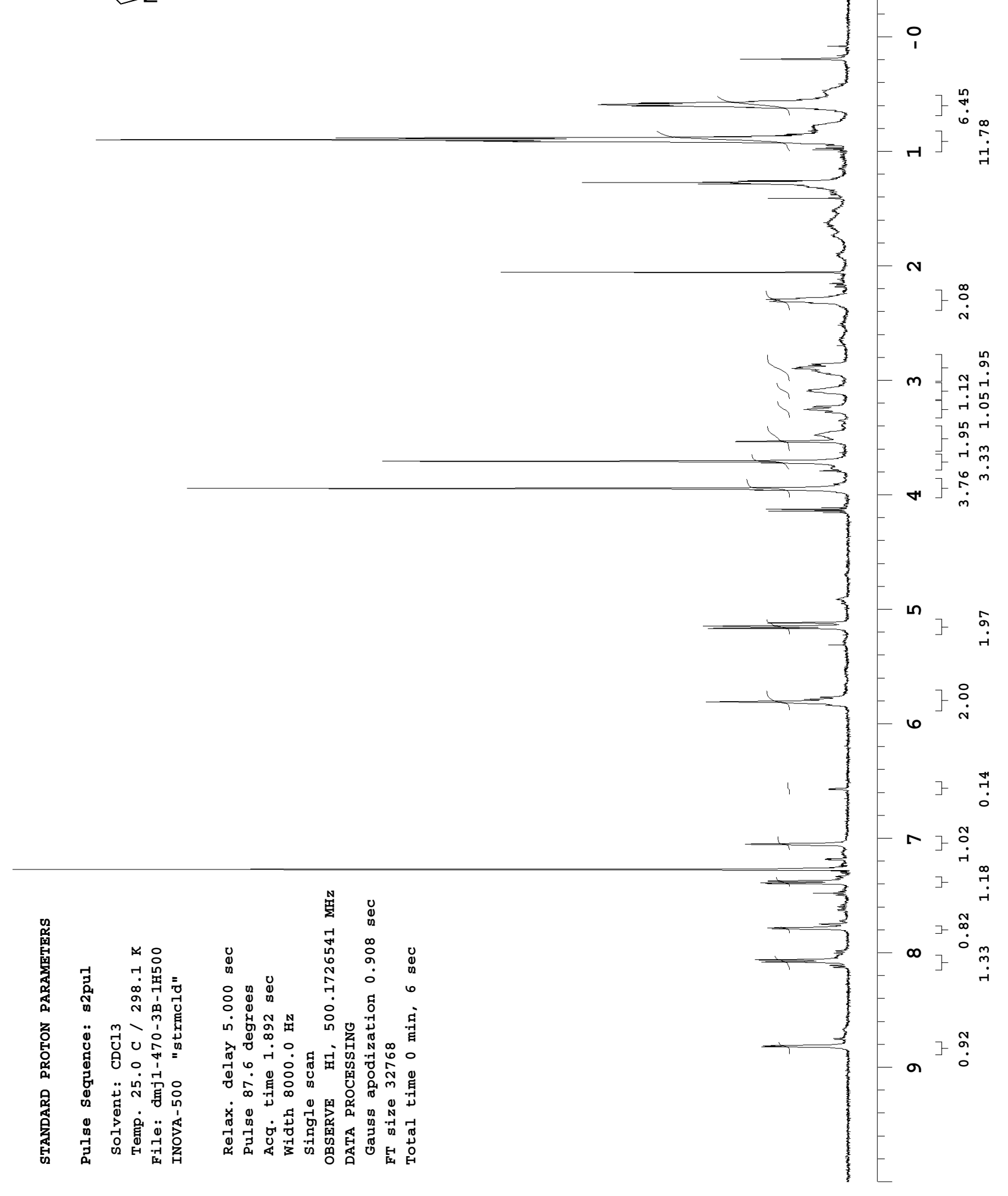


Page S90
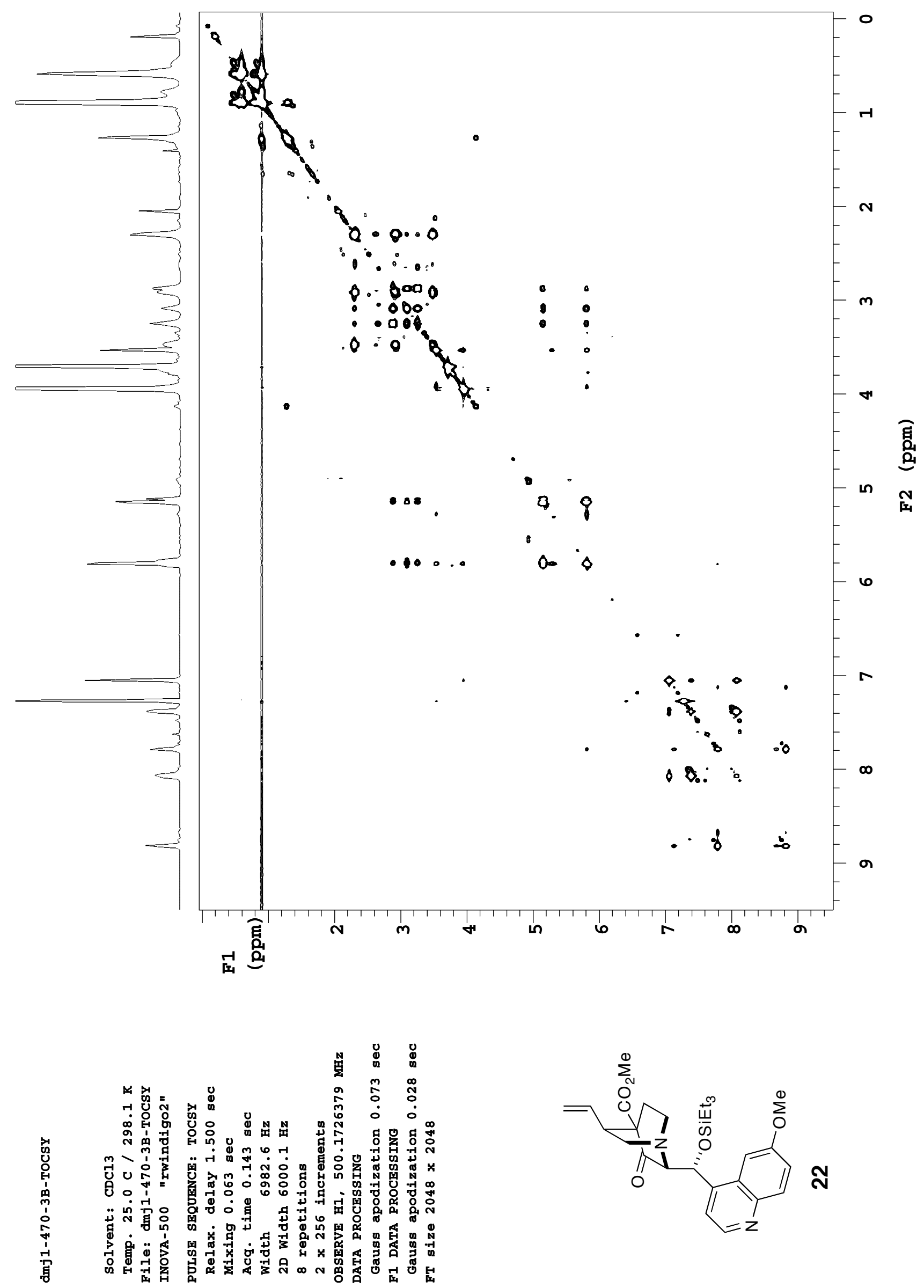
Page 591
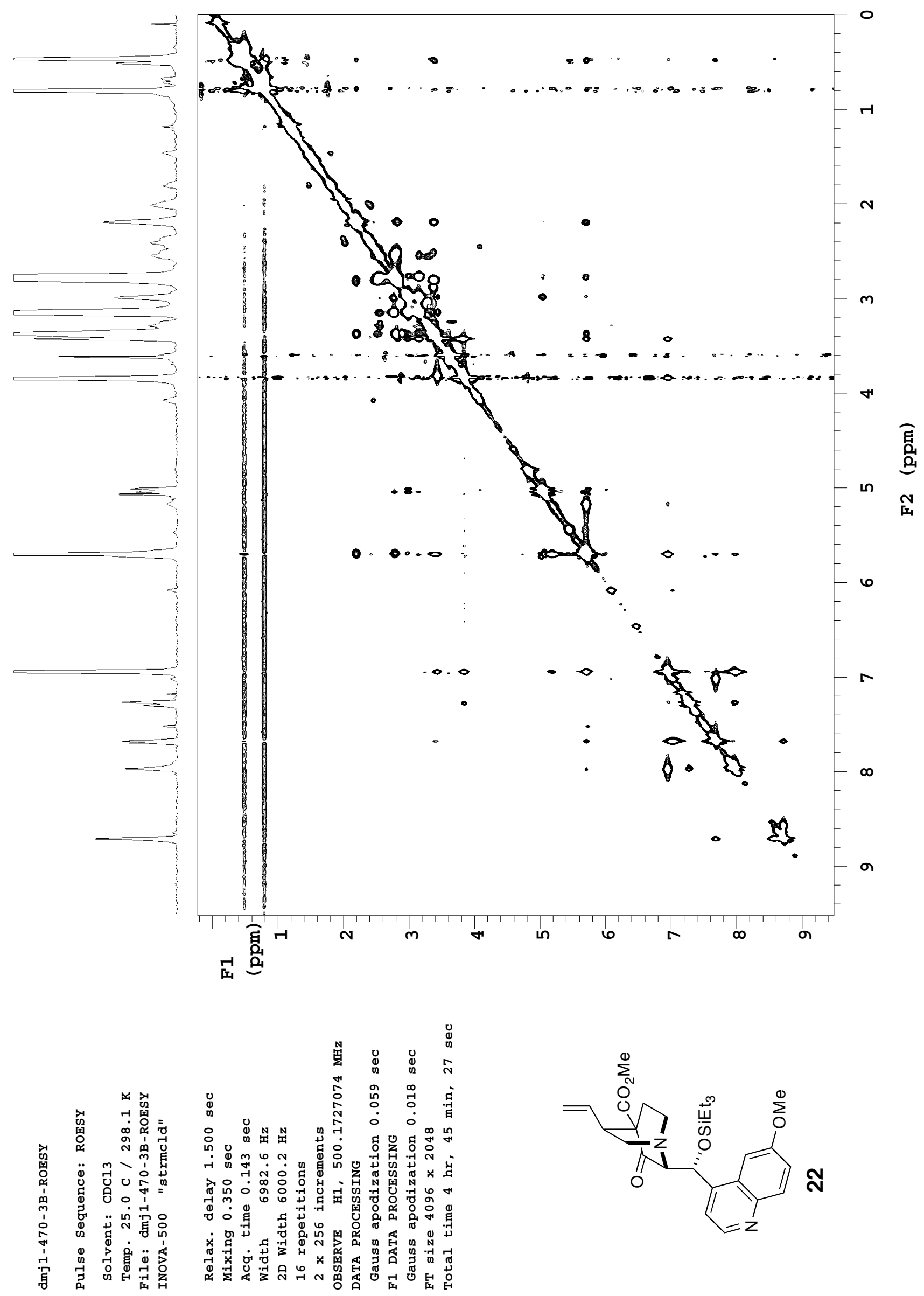
Page S92
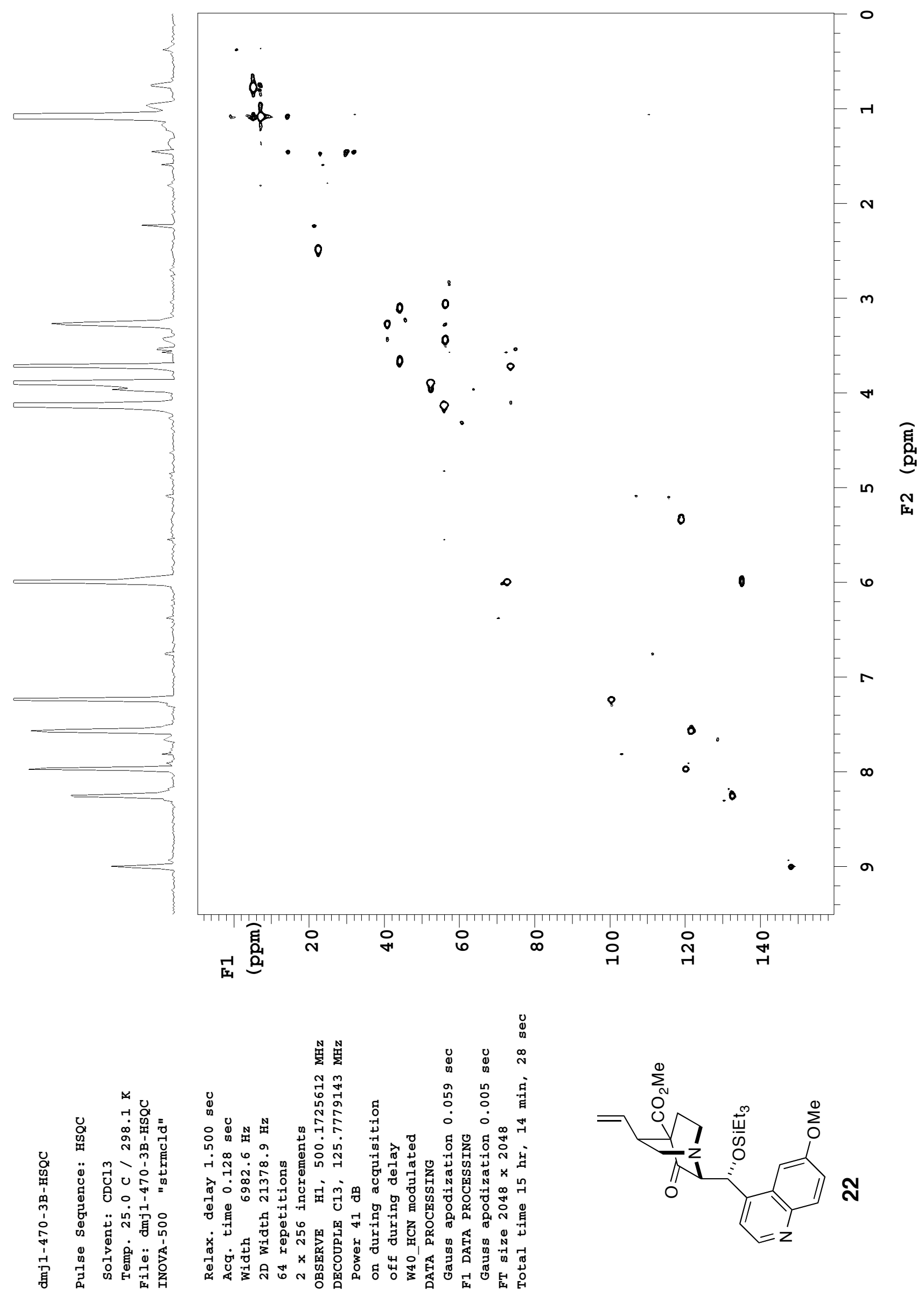


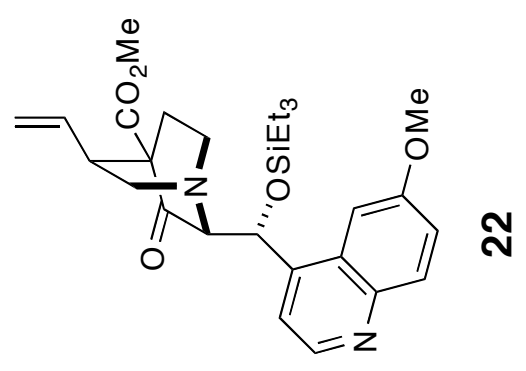

Page S93

ह్

ส

$\mathrm{St \tau} \cdot \mathrm{s}$

$020^{\circ} L$

$0 Z 6^{\circ} 9 L$

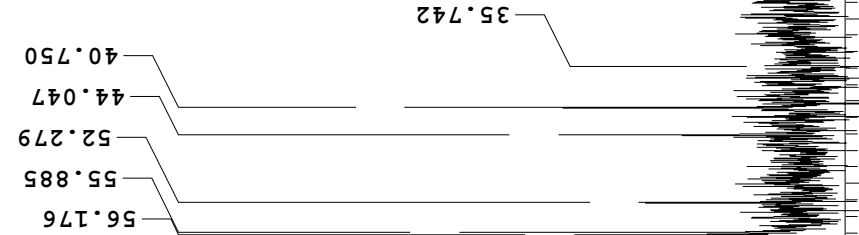

๑ $8^{\circ} L S$

$279^{\circ} z$

$097 \varepsilon L$

0हZ $\angle L-$

8SS. LL -

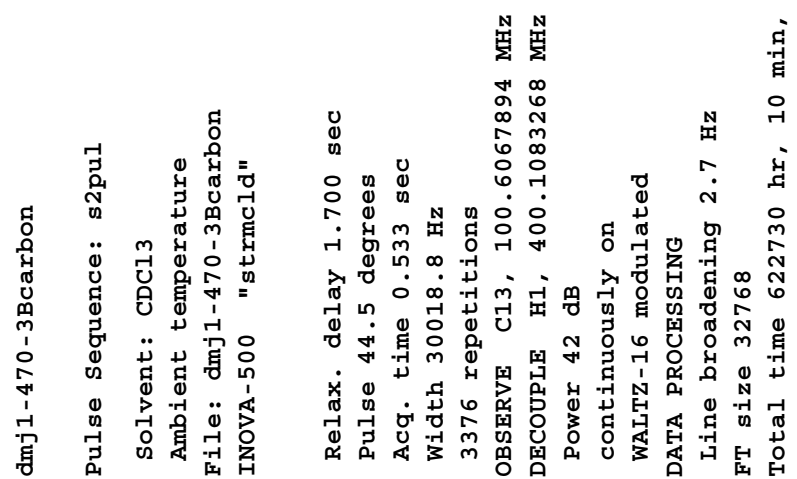

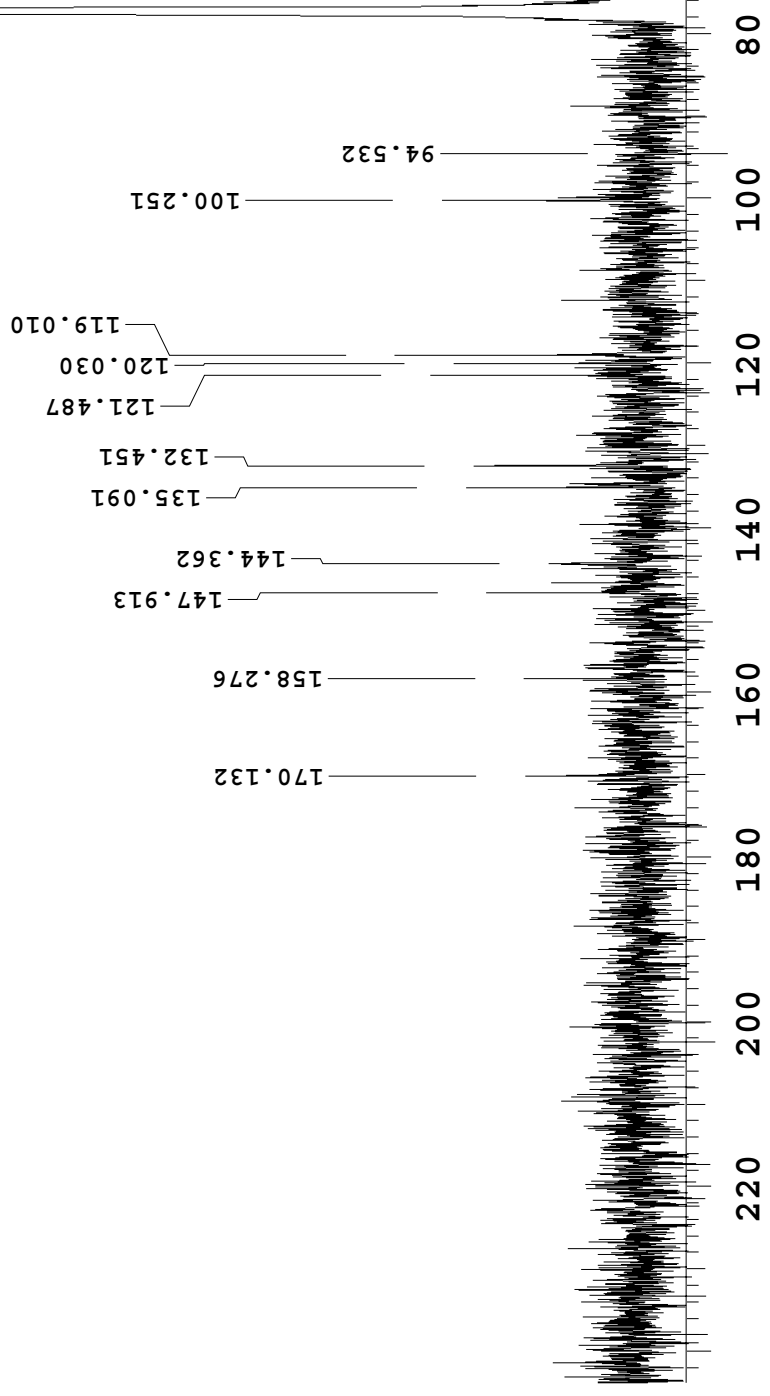




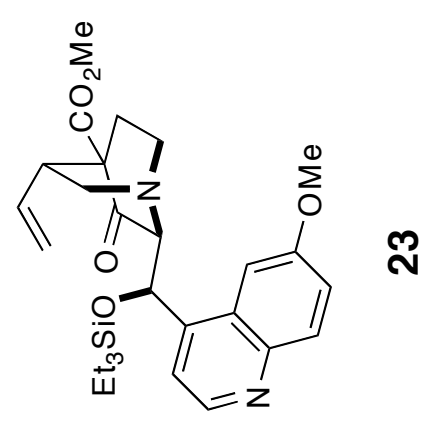

Page S94

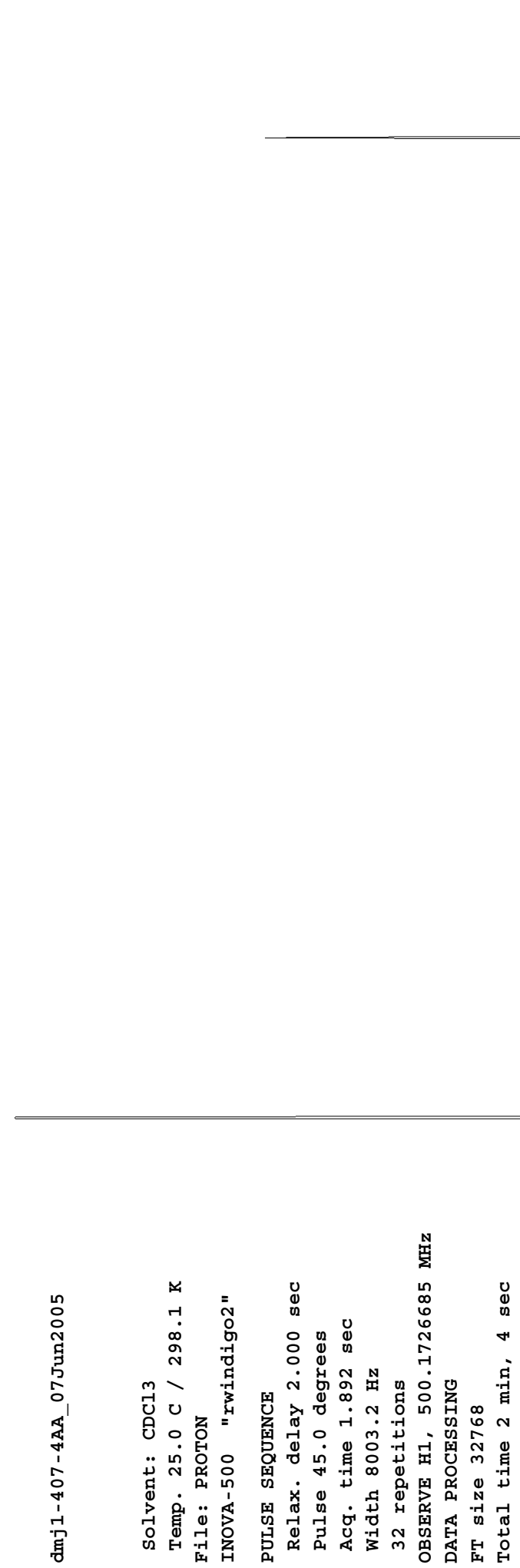


Page S95
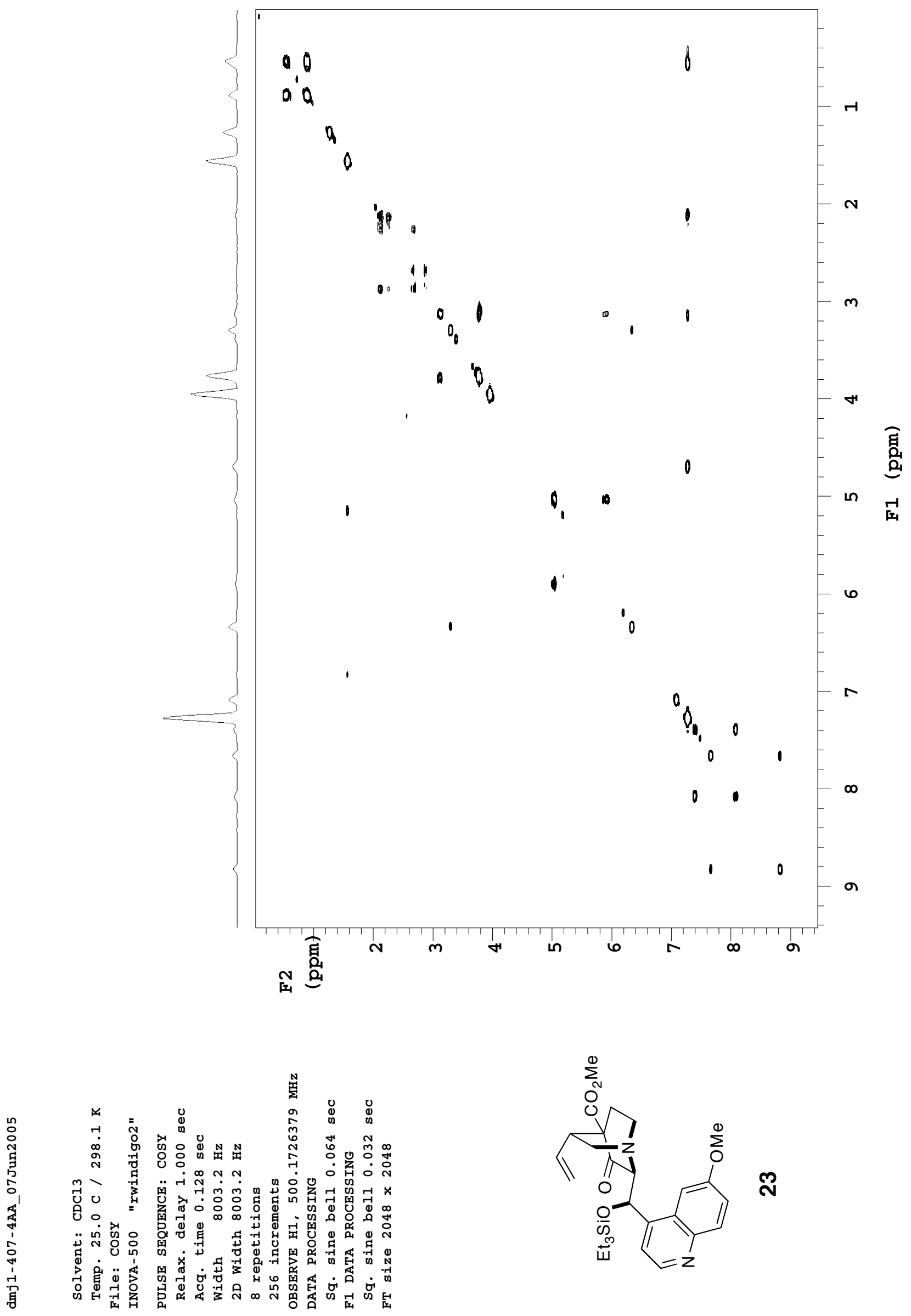
Page S96
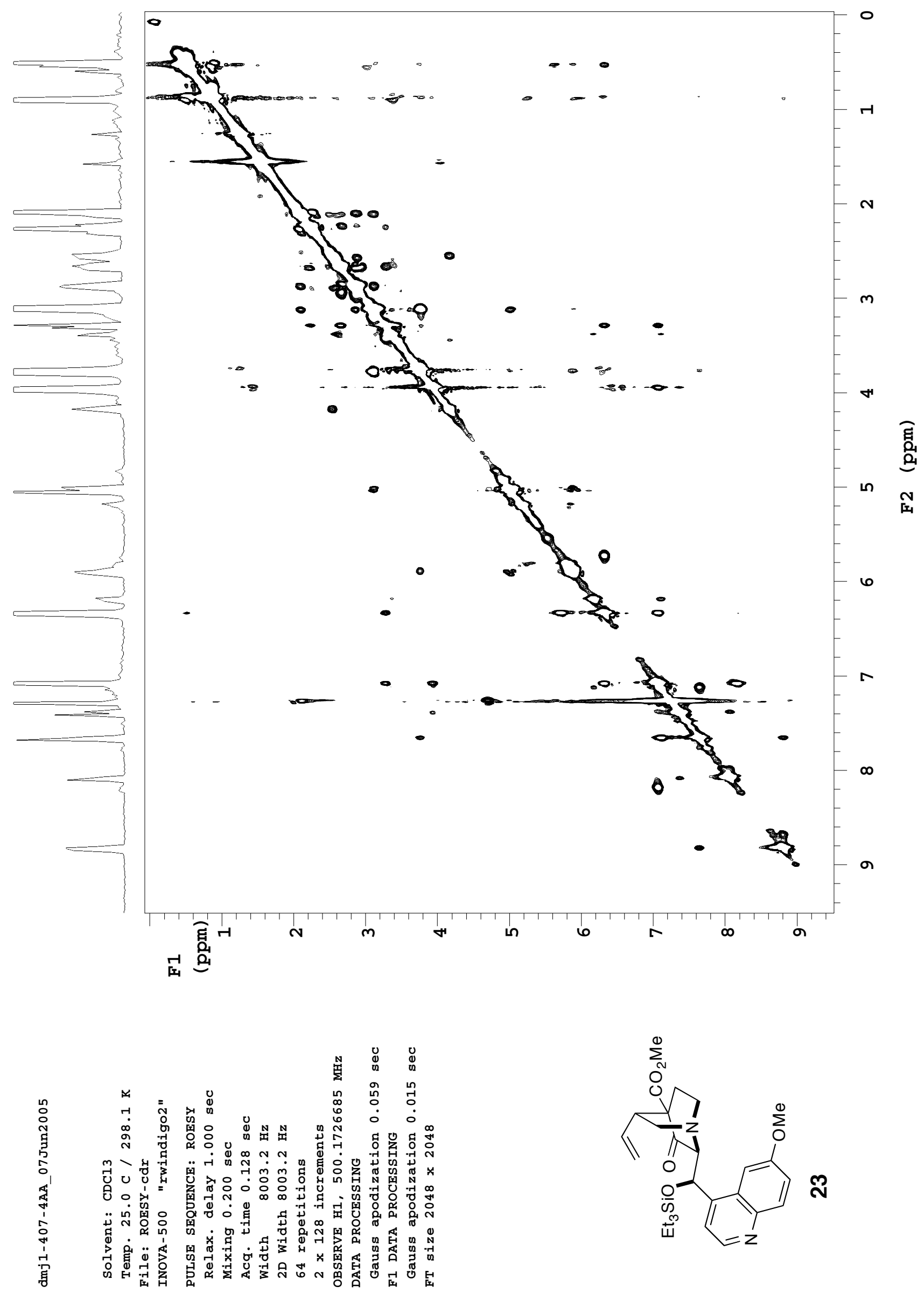
Page S97
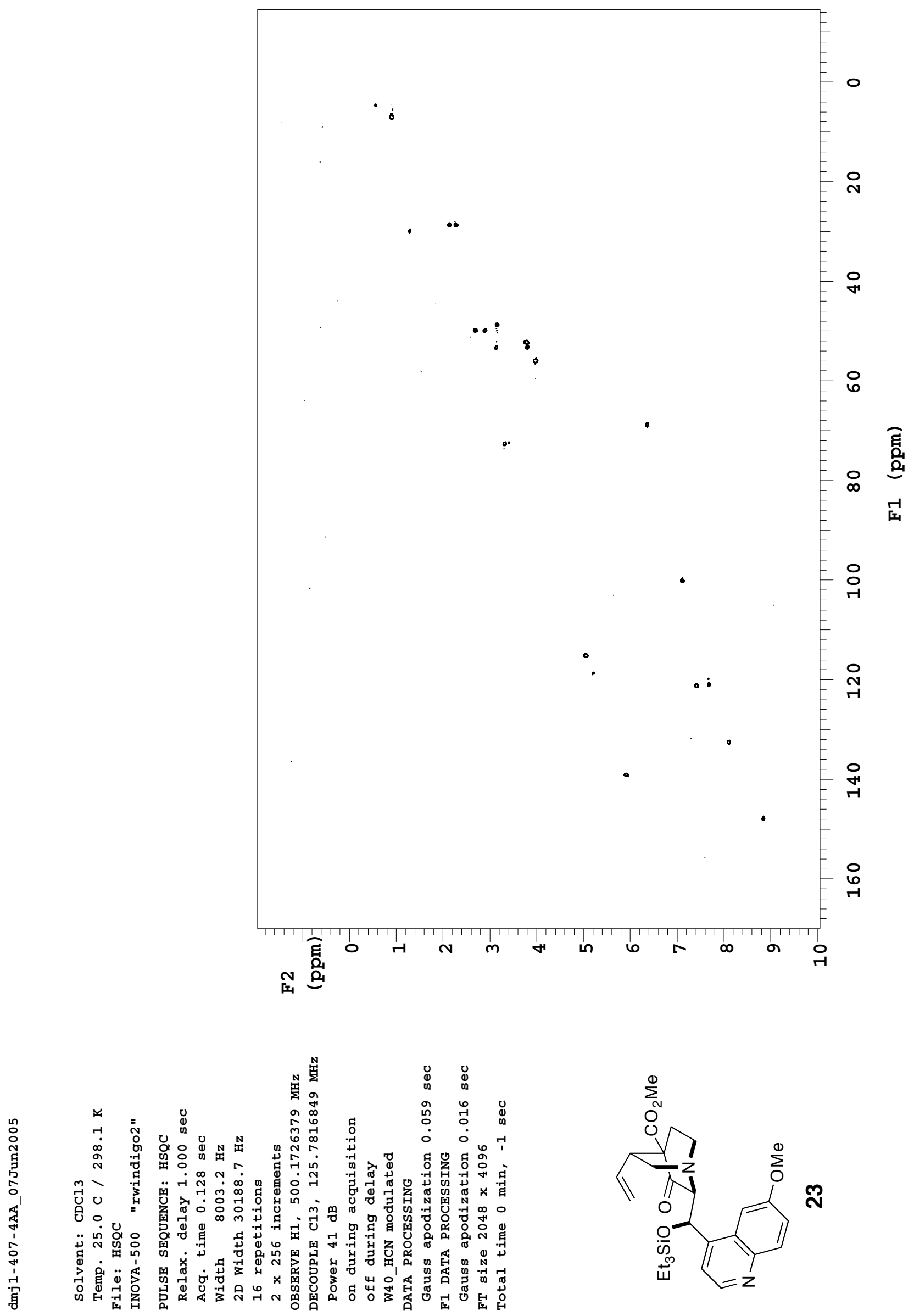

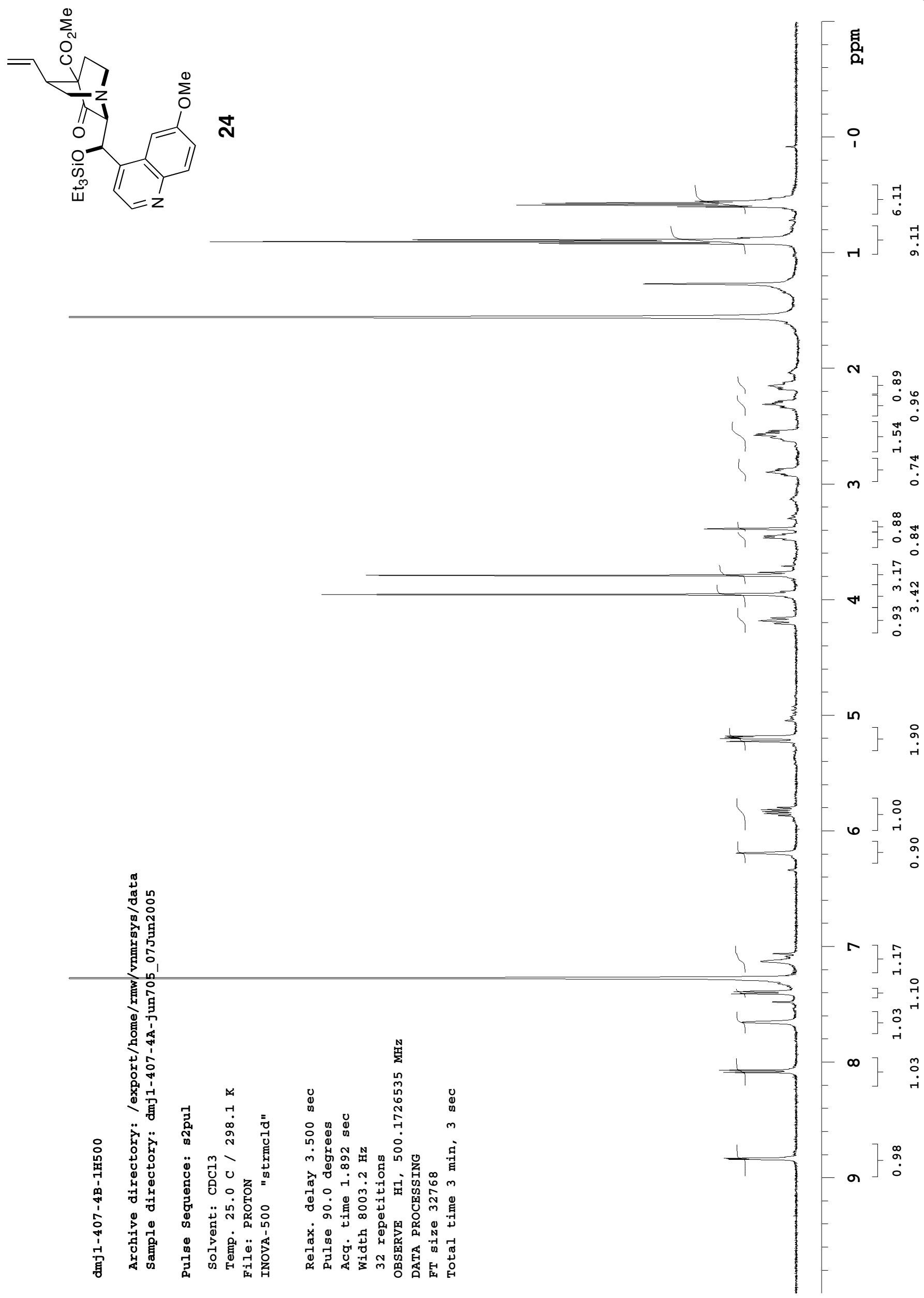

్ㅕㅁ

0

$\sqrt{-r} \stackrel{-}{:}$

- $5 \quad \begin{aligned} & -1 \\ & \sigma\end{aligned}$ 
Page S99

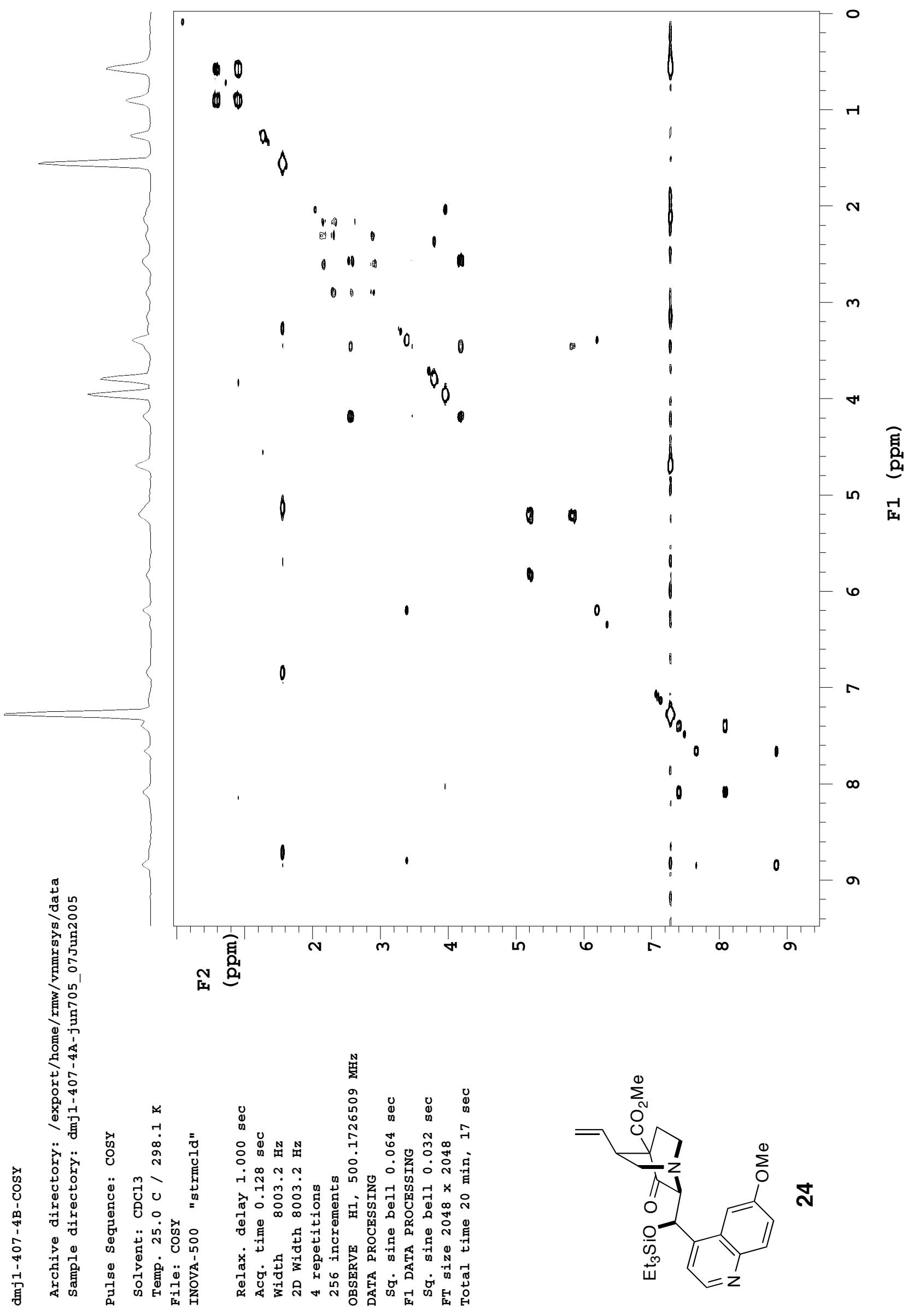


Page S100
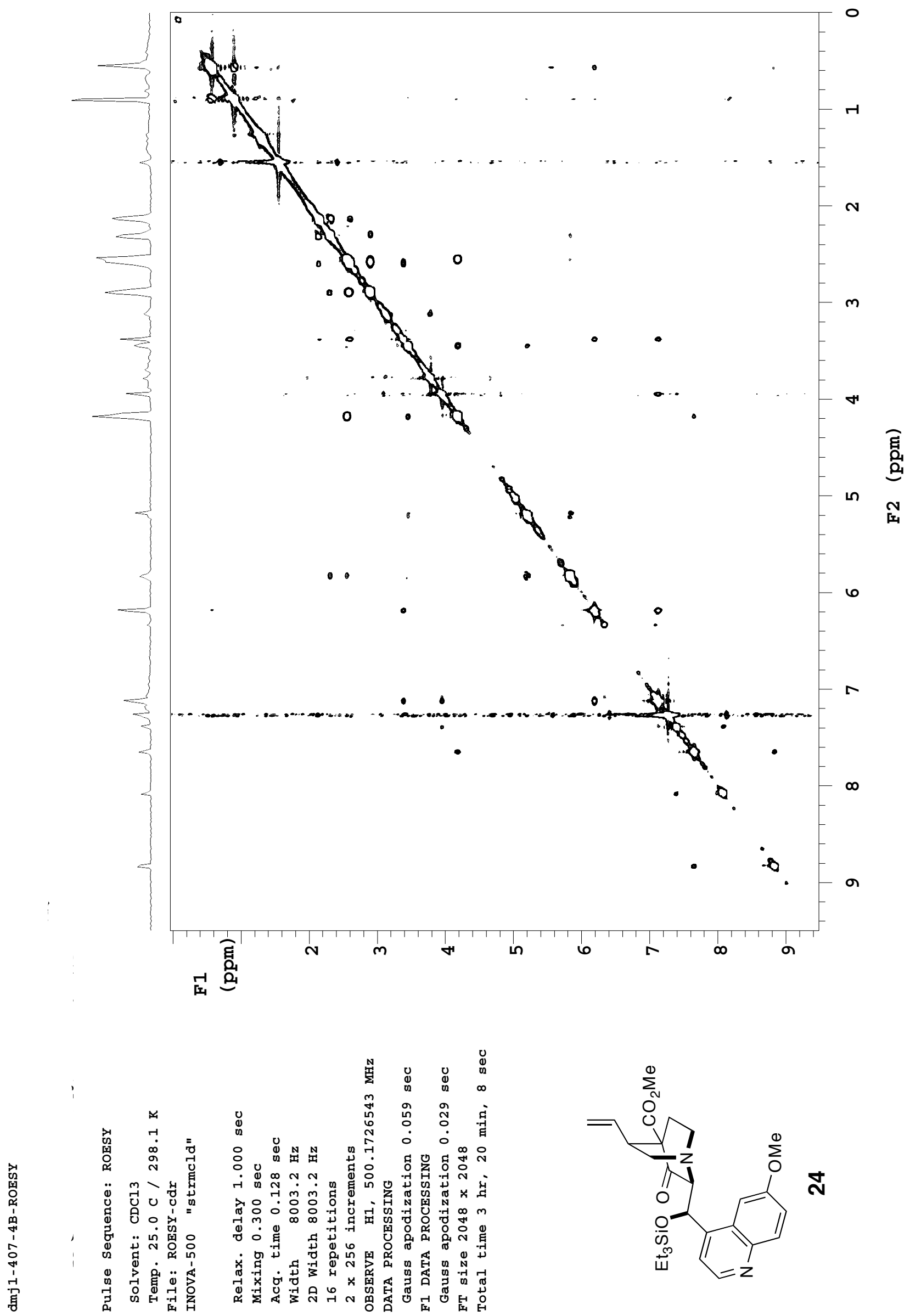
Page S101
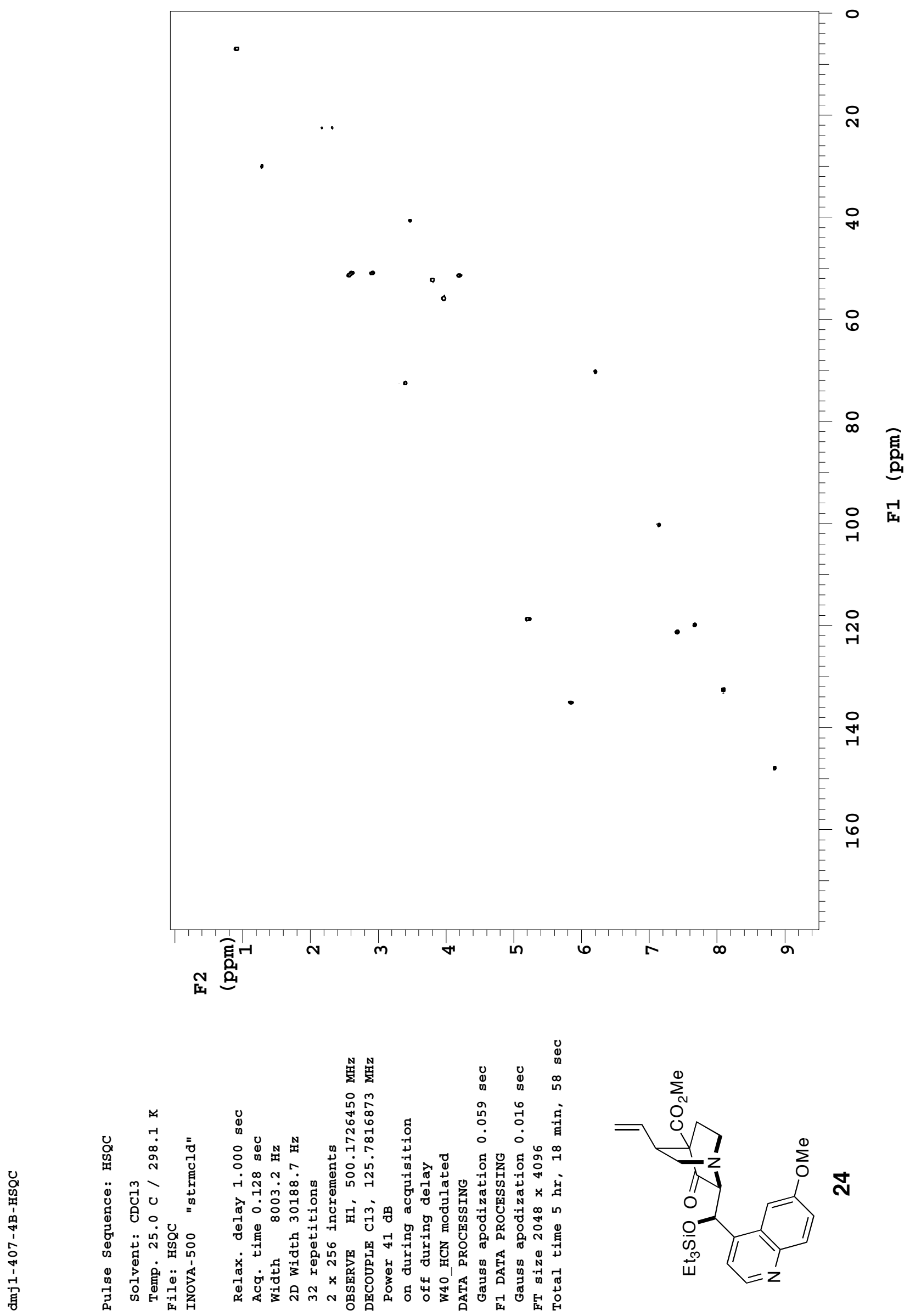
Page S102
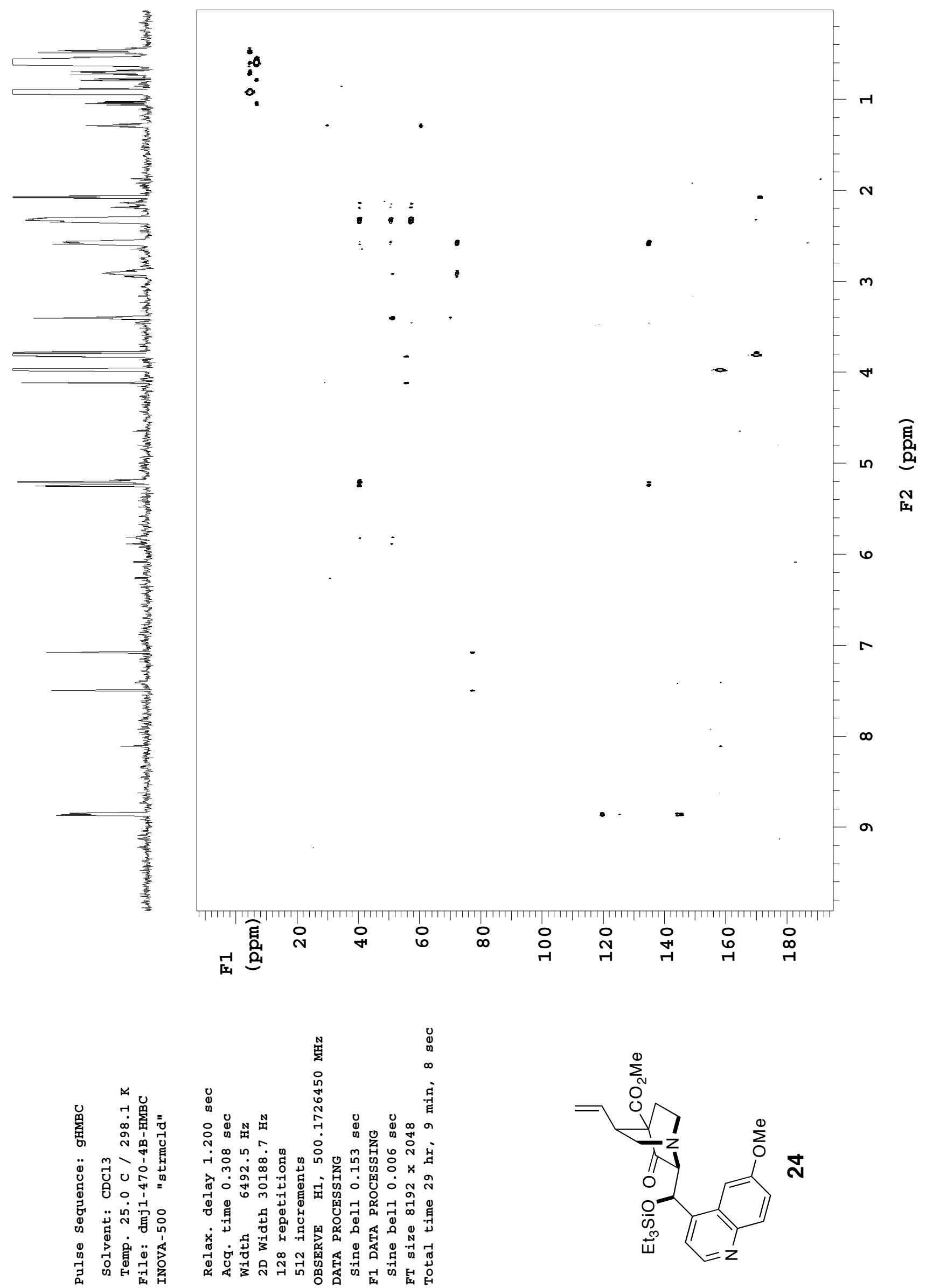

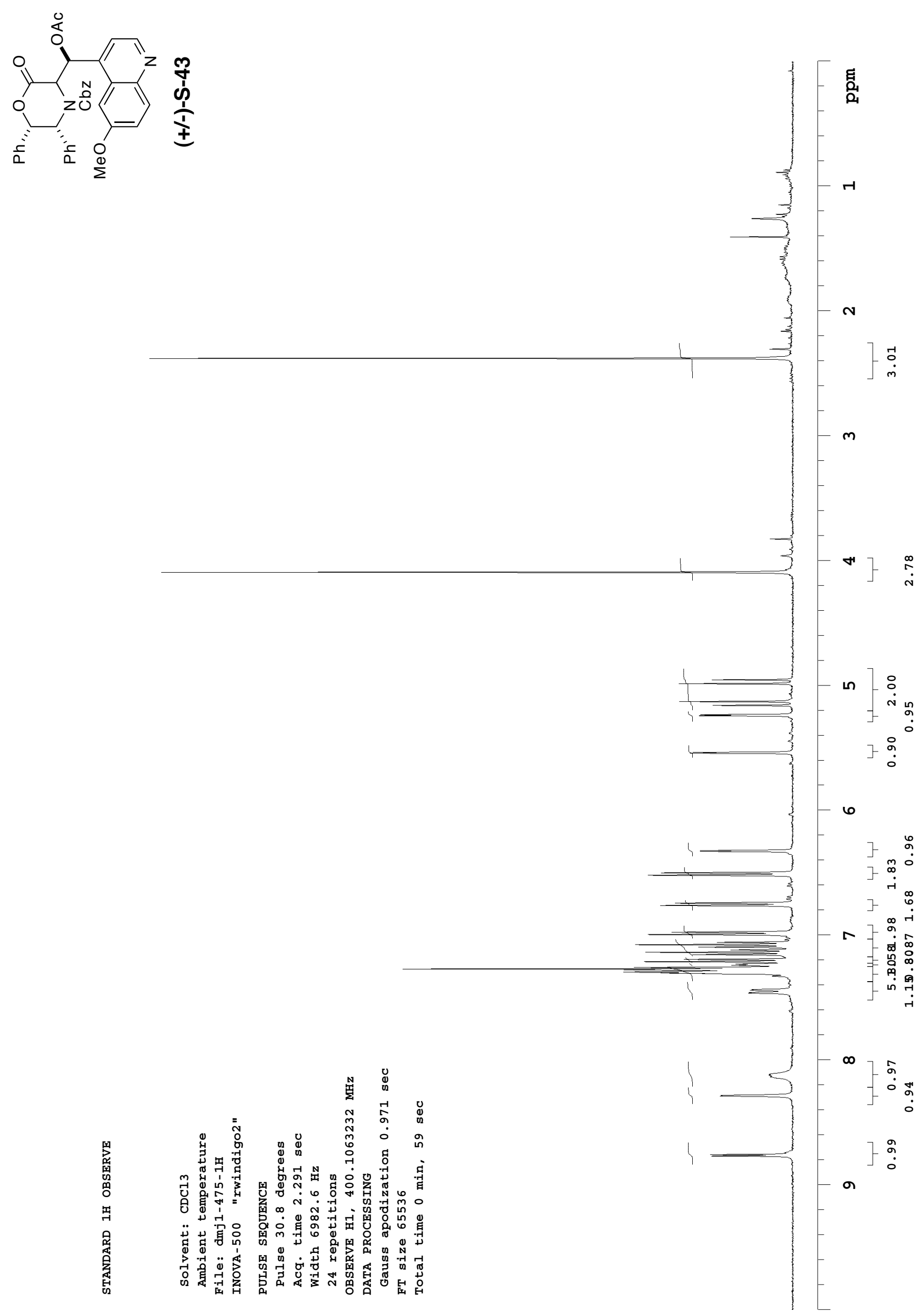

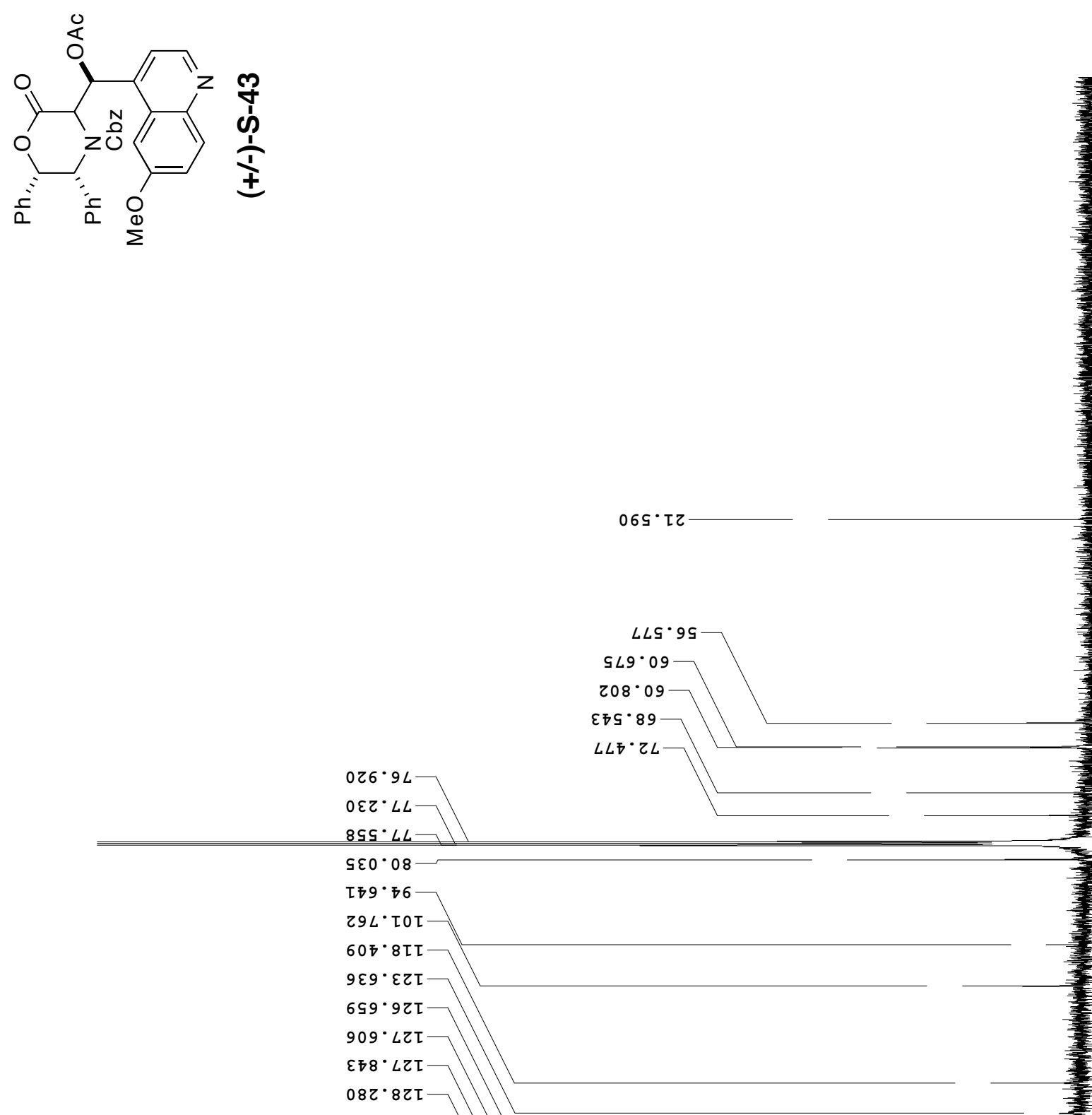

$08 Z \cdot 8 Z \tau$ $9 \tau \varepsilon \cdot 8 Z \tau$

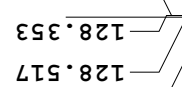
LIS'8ZT-

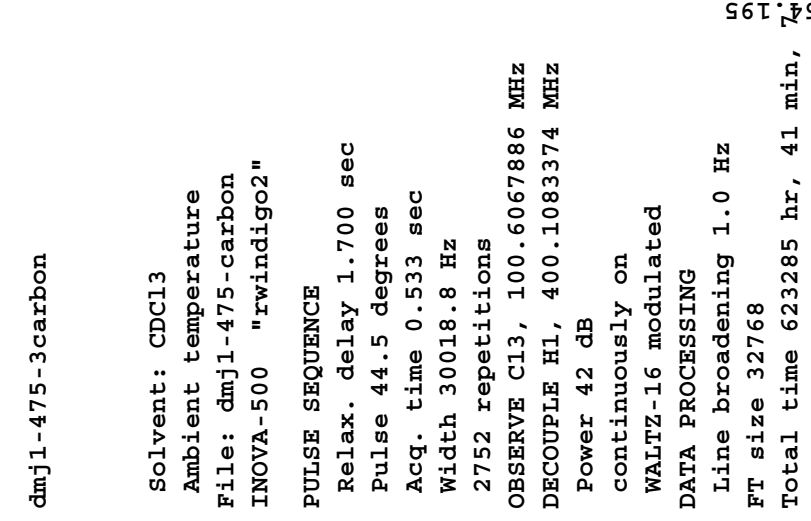

吕 

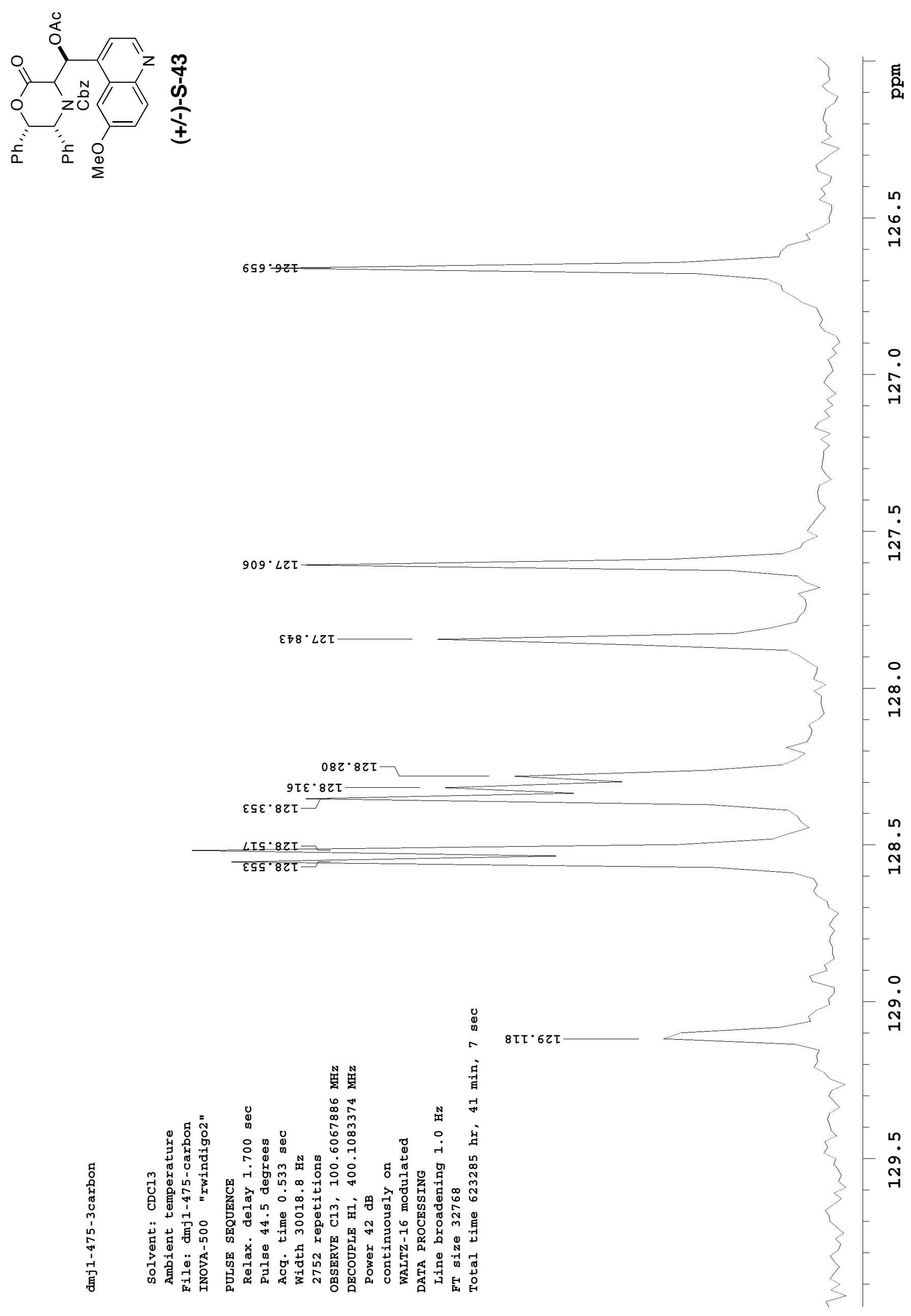